\title{
DEVELOPMENT OF IN VITRO TISSUE ENGINEERED BLOOD VESSEL MIMICS IN COMPLEX GEOMETRIES FOR CORONARY STENT TESTING
}

\author{
A Thesis Presented to the Faculty of \\ California Polytechnic State University, San Luis Obispo
}

In Partial Fulfillment of the Requirements for the Degree

Master of Science in Biomedical Engineering

By

Robert Dalton Chavez

June 2012 
(C) 2012 Robert Dalton Chavez

ALL RIGHTS RESERVED 


\section{Committee Membership}

Title:

Author:

Date Submitted:

Committee Chair:

Committee Member:

Committee Member:
Development of In Vitro Tissue Engineered Blood Vessel

Mimics in Complex Geometries for Coronary Stent Testing

Robert Dalton Chavez

June 7, 2012

Kristen O’Halloran Cardinal, Ph.D.

Trevor Ryan Cardinal, Ph.D.

Lily Hsu Laiho, Ph.D. 


\begin{abstract}
Development of In Vitro Tissue Engineered Blood Vessel Mimics in Complex Geometries for Coronary Stent Testing

Robert Dalton Chavez
\end{abstract}

Coronary heart disease is the leading cause of death in the United States and occurs when plaque occludes coronary arteries. Coronary stents, which may be used to treat coronary occlusions, are small metal tubes that are implanted in coronary arteries to restore blood flow. After stent implantation, endothelial cells grow over the stent so that blood contacts the endothelial cells instead of the stent surface; this event is known as reendothelialization. Re-endothelialization prevents blood from clotting on the stent surface and is a good predictor of stent success. Blood vessel mimics (BVMs) are in vitro tissue engineered models of human blood vessels that may be used to preclinically test coronary stents for re-endothelialization. BVMs have been developed in straight geometries, but the FDA has recommended that coronary devices be preclinically tested in complexshaped simulated vessels when the complex geometries of coronary arteries may negatively affect device performance. Coronary geometries may negatively affect the tissue response to coronary stents, therefore BVMs should be developed in complex geometries.

The goal of this thesis research was to fully develop complex-shaped scaffolds and bioreactors, to develop complex-shaped BVMs with cells located throughout all regions of the BVMs, and to develop a complex-shaped BVM with a confluent region of 
cells. First, bioreactors that can house complex-shaped scaffolds were designed, constructed, and validated. Complex-shaped BVMs were then developed by depositing cells throughout the entire inner surface of complex-shaped scaffolds, and the average and median cell densities throughout all regions of the BVMs were shown to be approximately the same order of magnitude as endothelial cell densities in native blood vessels. A stent was then successfully deployed in a complex-shaped BVM. The complex-shaped BVM straightened out to conform to the stent, which also occurs in native blood vessels. Finally, a confluent region of cells was developed on a complexshaped scaffold. Complex-shaped BVMs could eventually be used to preclinically test coronary stents, coronary drug-delivery systems, coronary imaging modalities, and other intravascular technologies. 


\section{Acknowledgements}

Drs. Trevor Cardinal and Lily Laiho, thank you for providing me with great suggestions and direction over the past few years. Sara Leifer, Aubrey Smith, Kasim Shah, Marcus Foley, Sarah Ur, the rest of the Cal Poly Tissue Engineering Laboratory, and Paige Czarnecki, you are the most incredible students on the Cal Poly campus. Sara, thank you for getting me started on the complex-shaped BVM project. Aubrey, thank you for helping me with many aspects of my research since 2008! Kasim, thank you for characterizing the multifunctional bioreactors. Marcus, thank you for helping me with a whole range of research-related tasks. Sarah, thank you for great suggestions on BVM tissue engineering. Paige, thank you for processing my many samples!

Mom (Nancy Walker Chavez), Dad (Robert Trias Chavez), and Dyl (my brother, Dylan Walker Chavez), you provided me with indispensable love and support. I don't know if I could have done it without you. Thank you.

Kristen, the laboratory experience you provided me, the endless list of other incredible opportunities you provided me, and the many conversations we've had together revealed a world of tissue engineering and research that I never would have discovered on my own. I love tissue engineering and research because of you. Thank you.

"Science is a very effective methodology for understanding partial truth, and I mean that very respectfully. Science doesn't look at the world as it is; science looks at the world as it interacts with the human system and certain questions that human beings ask."

- Deepak Chopra, M.D., F.A.C.P. 


\section{Table of Contents}

List of Tables.......................................................................................................

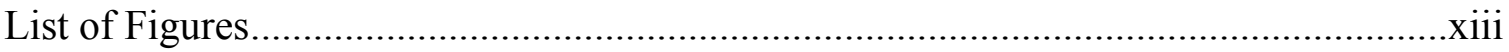

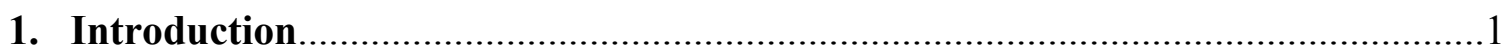

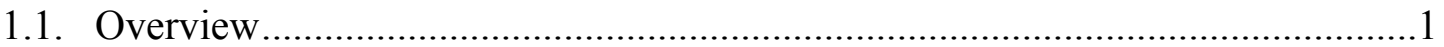

1.2. Coronary Heart Disease ........................................................................

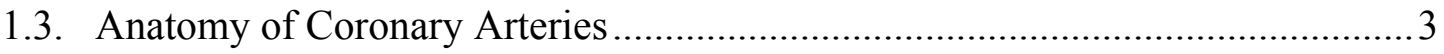

1.4. Blood Flow in Coronary Arteries ................................................................ 4

1.5. Shear Stress and Atherosclerosis in Coronary Arteries......................................

1.6. Devices for Identifying and Treating Coronary Heart Disease ........................ 8

1.6.1. Identifying Coronary Heart Disease ................................................... 8

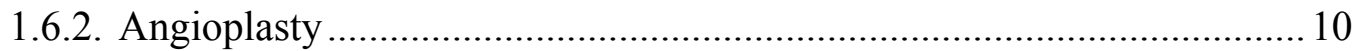

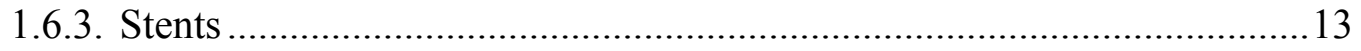

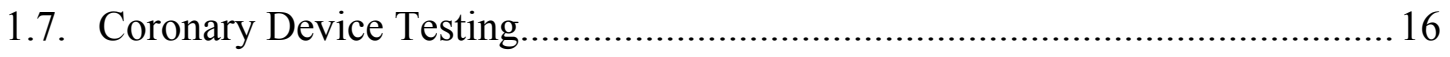

1.7.1. FDA Recommendations for In Vitro Studies........................................ 17

1.7.2. Synthetic Models of Coronary Vasculature ........................................ 17

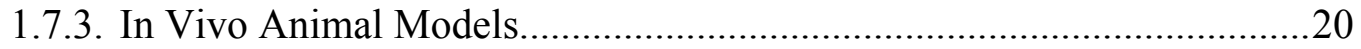

1.7.4. Blood Vessel Mimics .......................................................................... 23

1.8. Complex-Shaped Blood Vessel Mimics........................................................ 24

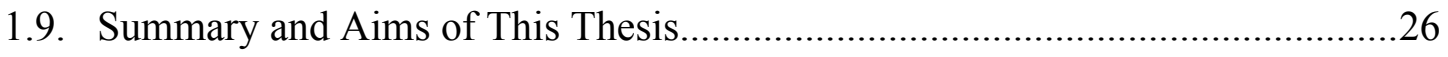




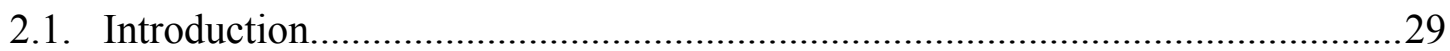

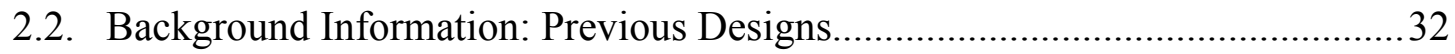

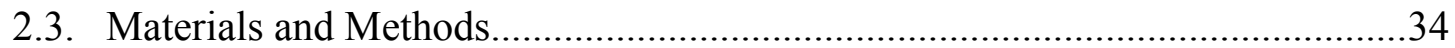

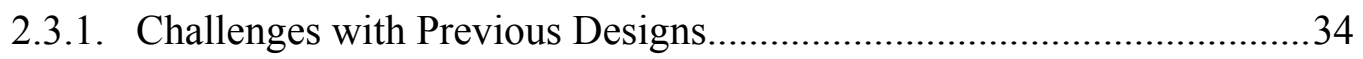

2.3.2. Final Designs for Complex-Shaped Scaffolds...................................... 35

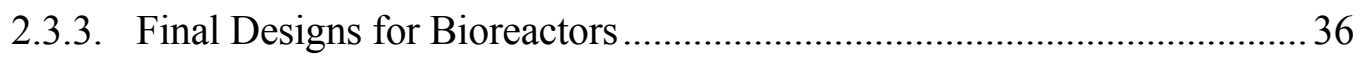

2.3.4. Construction of Complex-Shaped Scaffolds.......................................39

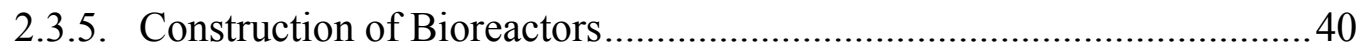

2.3.6. Validation of New Scaffolds and Bioreactors ....................................43

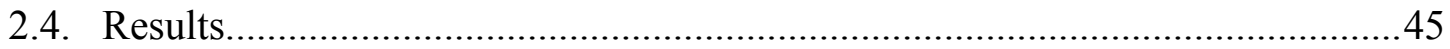

2.4.1. Prototypes of New Scaffolds and Bioreactors .................................45

2.4.2. Cell Deposition on Complex-Shaped Scaffolds ...................................48

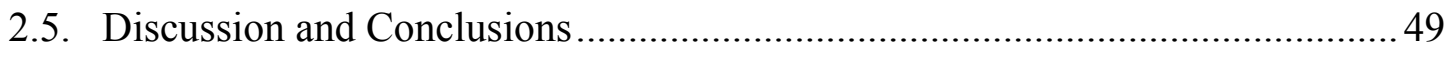

3. Consistency of Cell Deposition on Complex-Shaped Scaffolds ..........................51

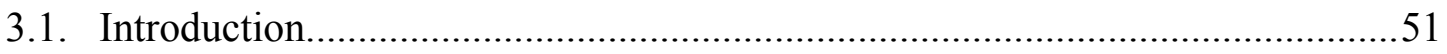

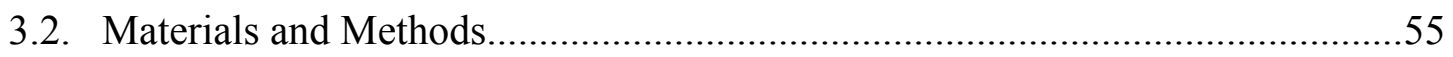

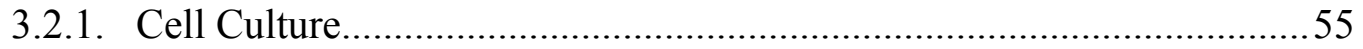

3.2.2. Sterilization and Media Preparation..................................................56

3.2.3. Denucleation and Protein-Coating of Scaffolds .................................57

3.2.4. Insertion of Scaffolds into Bioreactors and Priming.............................57

3.2.5. Pressure-Sodding of HUVECs...................................................... 61

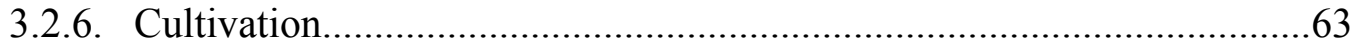


3.2.7. Harvesting and Fixation........................................................................ 63

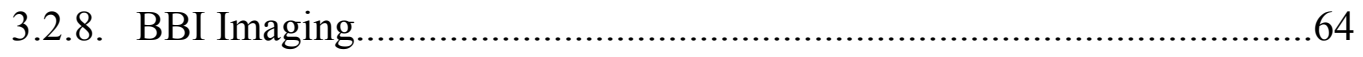

3.2.9. Cell Counting in BBI Images...............................................................66

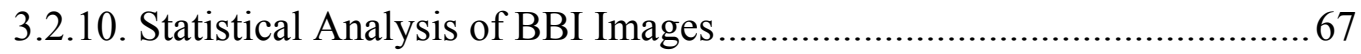

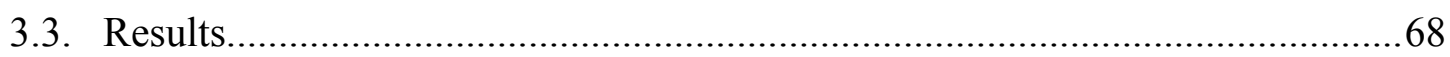

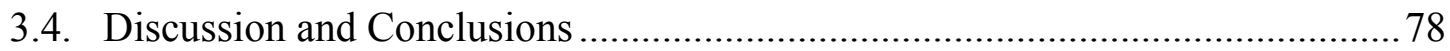

4. Effect of Flow Rate on Cell Confluence in U-Shaped BVMs...............................82

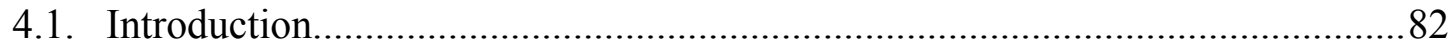

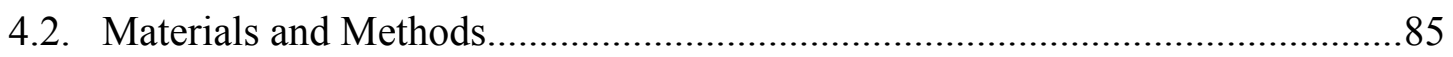

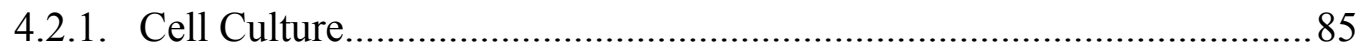

4.2.2. Sterilization and Media Preparation.........................................................86

4.2.3. Scaffold Preparation and Priming.......................................................... 86

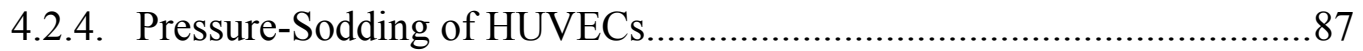

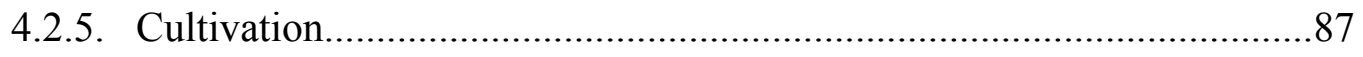

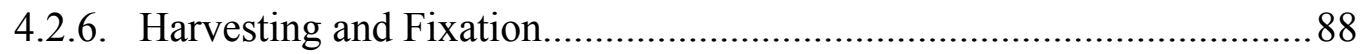

4.2.7. Histologic Analysis, BBI Imaging, and SEM Imaging............................89

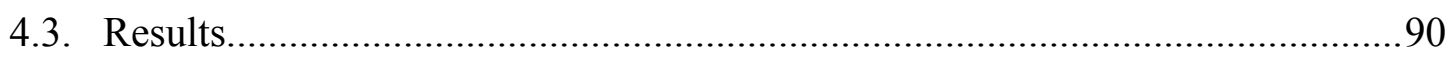

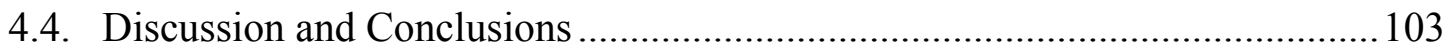

5. Possible Cell Denudation After Stent Deployment in a U-Shaped BVM............109

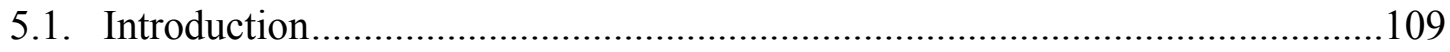

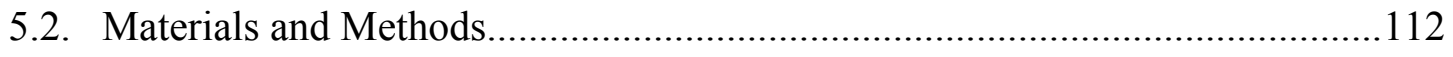

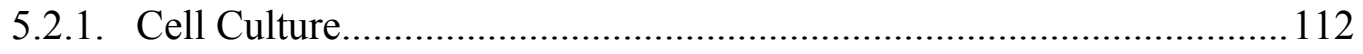

5.2.2. Scaffold Sterilization, Scaffold Preparation, and Priming.....................112 


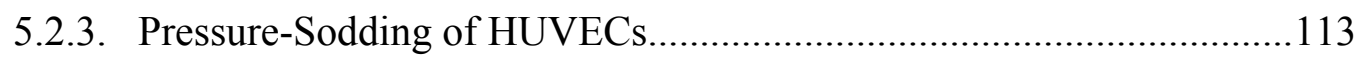

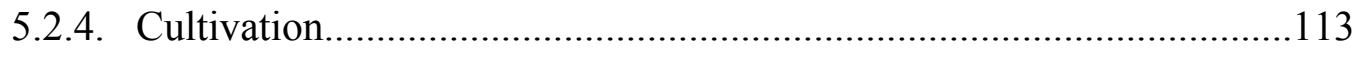

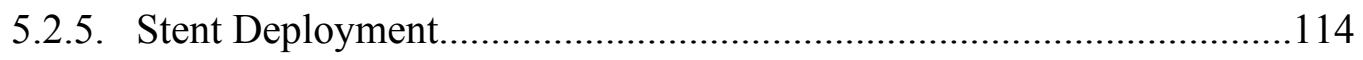

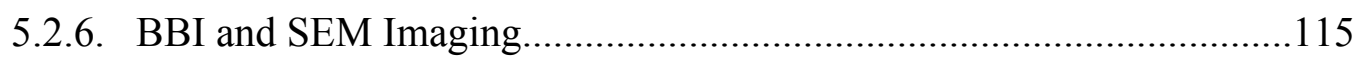

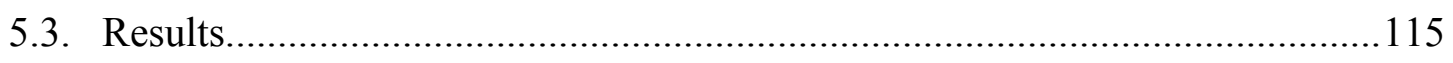

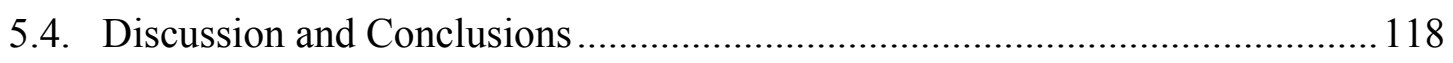

6. Development of a Confluent Region of Cells in a U-Shaped BVM .....................122

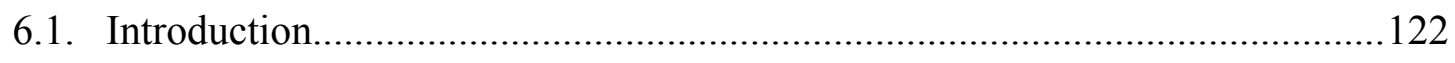

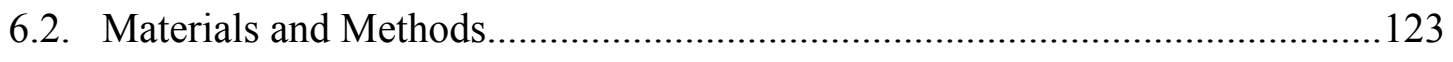

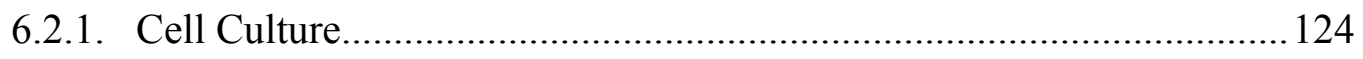

6.2.2. Scaffold Sterilization, Scaffold Preparation, and Priming.....................125

6.2.3. Pressure-Sodding of HUVSMCs ........................................................ 125

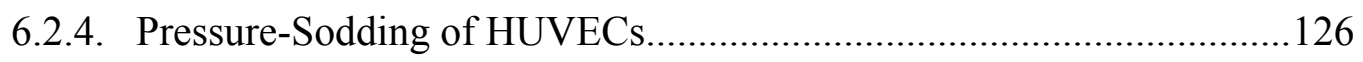

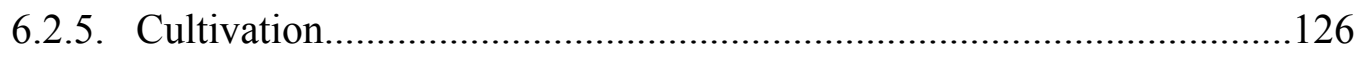

6.2.6. Histologic Analysis, Fluorescence Imaging, and SEM Imaging...........127

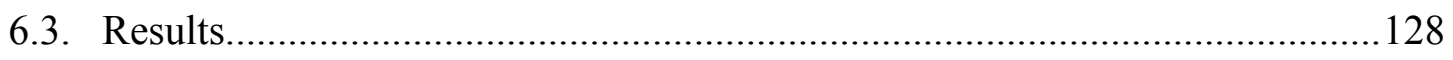

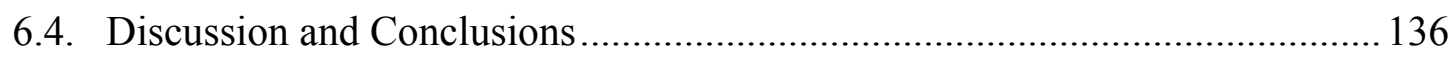

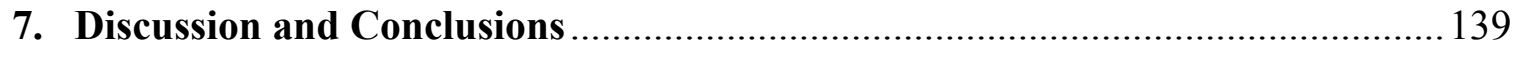

7.1. Review of the Purpose of Complex-Shaped BVMs ..........................................139

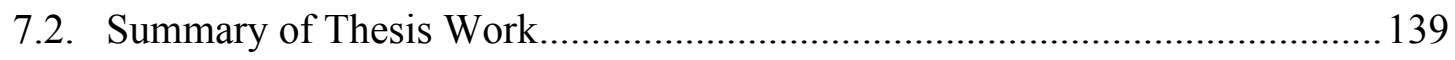

7.3. Limitations and Future Work....................................................................... 142

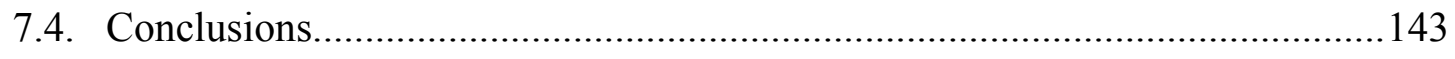

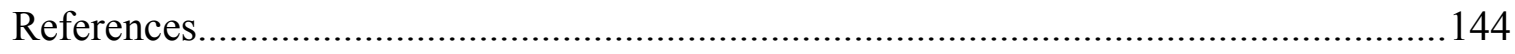


Appendix A: Abbreviations

Appendix B: Protocol for Maintaining Multifunctional Bioreactors.................................157

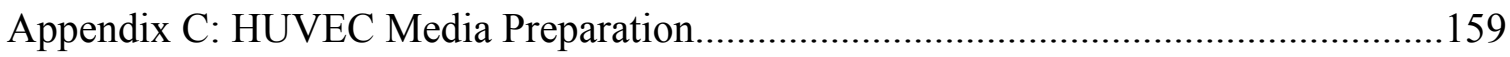

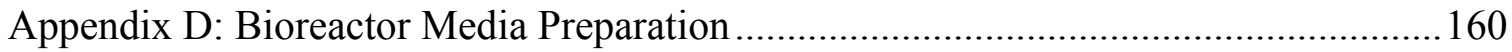

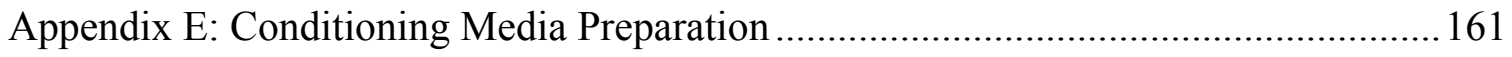

Appendix F: Complex-Shaped BVM Harvesting Protocol ..............................................162

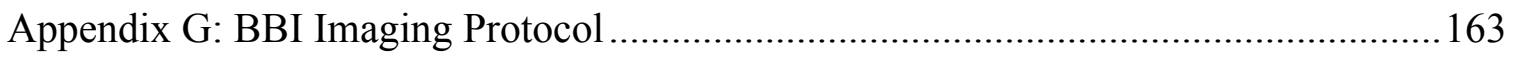

Appendix H: Protocol for Developing HUVEC-Sodded Complex-Shaped BVMs .........166

Appendix I: Raw Data for Statistical Analysis of Cell Deposition................................... 168

Appendix J: Minitab Output for Statistical Analysis of Cell Deposition.......................... 176

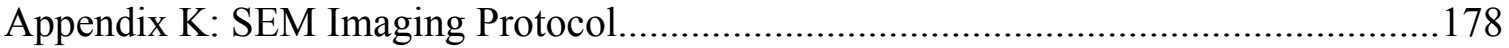

Appendix L: Histology and White Light Imaging Protocol ...........................................179

Appendix M: HUVSMC Media Preparation ................................................................183

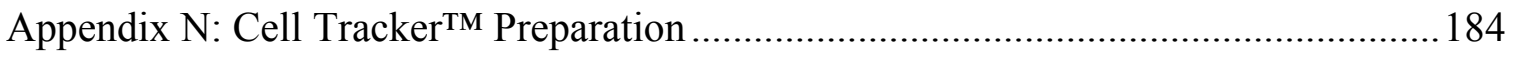

Appendix O: Protocol for Developing Dual-Sodded Complex-Shaped BVMs ...............185

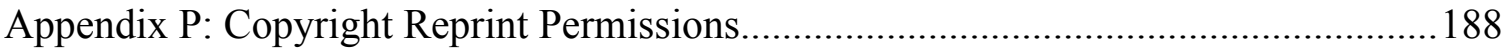




\section{List of Tables}

Table 2.1. Materials for Multifunctional Bioreactor.................................................... 42

Table 2.2. Materials for Bioreactor Capable of Housing Only Y-Shaped Scaffolds.......43

Table 3.1. Average Cell Counts $\pm \mathrm{SD}$ for an Area of $10,000 \mu \mathrm{m}^{2}$ Along Length

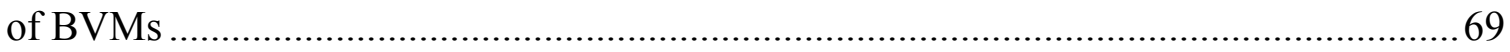

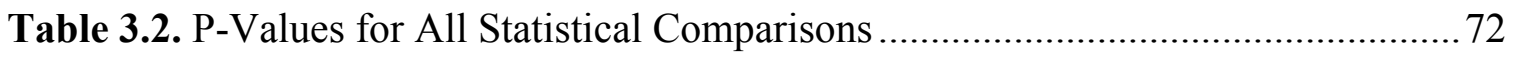

Table 4.1. Effect of Flow Rate on Confluence of Cell Lining in U-Shaped BVMs .........90

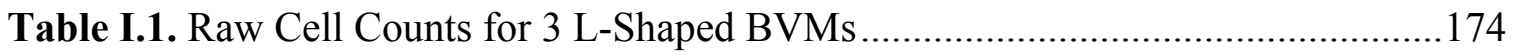

Table I.2. Raw Cell Counts for 3 U-Shaped BVMs ................................................ 175 


\section{List of Figures}

Figure 1.1. Anatomy of human coronary arteries............................................... 4

Figure 1.2. Schematics of complex-shaped coronary arteries ................................... 5

Figure 1.3. Plaque development in bent coronary arteries........................................6

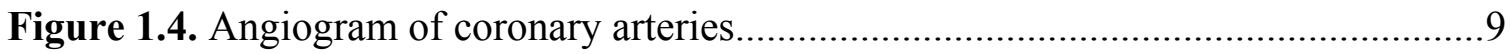

Figure 1.5. Placement, inflation, deflation, and removal of a balloon............................ 11

Figure 1.6. Deployment of a balloon-expandable stent in a coronary artery................... 14

Figure 1.7. A complex-shaped simulated vessel.................................................... 18

Figure 1.8. A physiologically accurate complex-shaped simulated vessel....................19

Figure 1.9. Re-endothelialization of a stent in a rabbit iliac artery .............................22

Figure 1.10. Re-endothelialization of a stent in a coronary bifurcation ........................25

Figure 2.1. Current BVM bioreactor housing a straight scaffold ...................................30

Figure 2.2. Current straight scaffold and pressure-sodding method.............................. 31

Figure 2.3. Construction methods for a T-shaped scaffold (A) and a Y-shaped

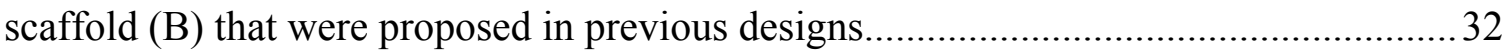

Figure 2.4. Previously proposed designs for bioreactors that can house complex-

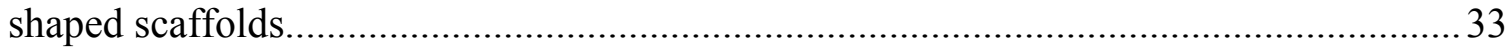

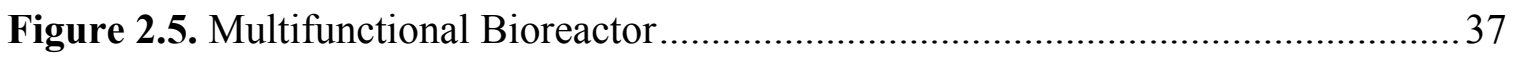

Figure 2.6. Bioreactor capable of housing only a Y-shaped scaffold............................ 38

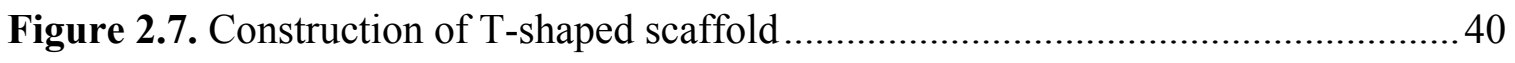

Figure 2.9. Prototypes of the new scaffolds and bioreactors .................................. 47

Figure 2.10. BBI image showing HUVEC deposition on U-shaped scaffold..................48 
Figure 3.1. Cross-sections of straight BVM

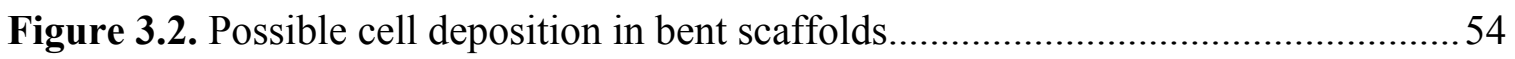

Figure 3.3. Insertion of $U$-shaped scaffold into bioreactor........................................59

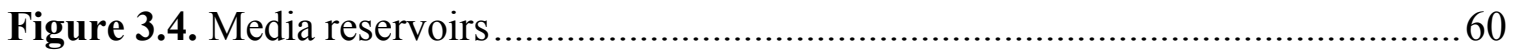

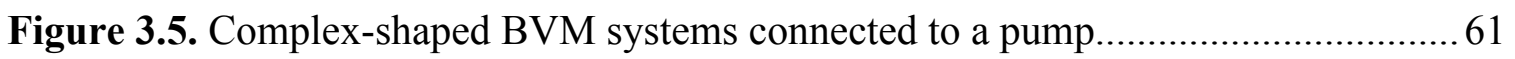

Figure 3.6. Cutting of L- and U-shaped BVMs into segments for evaluation..................65

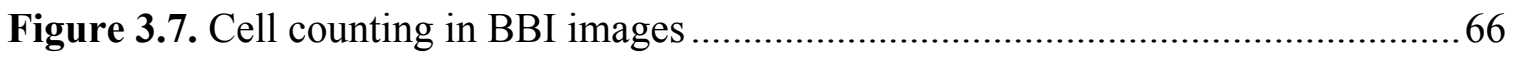

Figure 3.8. Sample BBI images from the 6 complex-shaped BVMs in this study..........69

Figure 3.9. Probability plots of data sets for L- and U-shaped BVMs ..........................71

Figure 3.10. Boxplot of cell density along length of L-shaped BVMs.......................... 74

Figure 3.11. Individual value plot of cell density along length of L-shaped BVMs .........74

Figure 3.12. Boxplot of cell density in inner and outer halves of L-shaped BVMs ........75

Figure 3.13. Boxplot of cell density in top and bottom halves of L-shaped BVMs........ 75

Figure 3.14. Boxplot of cell density along length of U-shaped BVMs .........................76

Figure 3.15. Individual value plot of cell density along length of U-shaped BVMs.........76

Figure 3.16. Boxplot of cell density in inner and outer halves of U-shaped BVMs........77

Figure 3.17. Boxplot of cell density in top and bottom halves of U-shaped BVMs........77

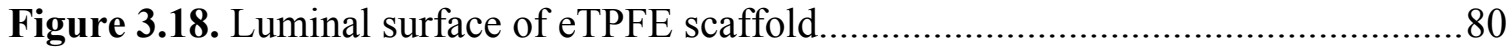

Figure 4.1. Histologic cross-section of a U-shaped BVM cultivated at $0 \mathrm{~mL} / \mathrm{min}$ for

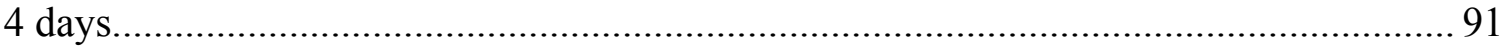

Figure 4.2. BBI image of a U-shaped BVM cultivated at $0 \mathrm{~mL} / \mathrm{min}$ for 4 days..............92

Figure 4.3. SEM image of a U-shaped BVM cultivated at $0 \mathrm{~mL} / \mathrm{min}$ for 4 days............ 92 
Figure 4.4. Histologic cross-section of a U-shaped BVM cultivated at $0 \mathrm{~mL} / \mathrm{min}$ for

7 days

Figure 4.5. BBI image of a U-shaped BVM cultivated at $0 \mathrm{~mL} / \mathrm{min}$ for 7 days. .94

Figure 4.6. SEM image of a U-shaped BVM cultivated at $0 \mathrm{~mL} / \mathrm{min}$ for 7 days 94

Figure 4.7. Histologic cross-section of a U-shaped BVM cultivated at an increasing

flow rate for 4 days 95

Figure 4.8. BBI image of a U-shaped BVM cultivated at an increasing flow rate for 4 days. 96

Figure 4.9. SEM image of a U-shaped BVM cultivated at an increasing flow rate for 4 days

Figure 4.10. Histologic cross-section of a U-shaped BVM cultivated at an increasing flow rate for 7 days.

Figure 4.11. BBI image of a U-shaped BVM cultivated at an increasing flow rate for 7 days.

Figure 4.12. SEM image of a U-shaped BVM cultivated at an increasing flow rate for 7 days 98

Figure 4.13. Histologic cross-section of a U-shaped BVM cultivated at a flow rate of $12 \mathrm{~mL} / \mathrm{min}$ for 4 days .99

Figure 4.14. BBI image of a U-shaped BVM cultivated at a flow rate of $12 \mathrm{~mL} / \mathrm{min}$ for 4 days 100

Figure 4.15. SEM image of a U-shaped BVM cultivated at a flow rate of $12 \mathrm{~mL} / \mathrm{min}$ for 4 days. 100 
Figure 4.16. Histologic cross-section of a U-shaped BVM cultivated at a flow rate of $12 \mathrm{~mL} / \mathrm{min}$ for 7 days

Figure 4.17. BBI image of a U-shaped BVM cultivated at a flow rate of $12 \mathrm{~mL} / \mathrm{min}$

for 7 days

Figure 4.18. SEM image of a U-shaped BVM cultivated at a flow rate of $12 \mathrm{~mL} / \mathrm{min}$

for 7 days

Figure 4.19. Cell loss after first day of cultivation of U-shaped BVMs.

Figure 5.1. Stent deployment in a U-shaped BVM 116

Figure 5.2. Separation of a U-shaped BVM from a rigid coronary stent. 116

Figure 5.3. BBI image of a U-shaped BVM with no implanted stent.

Figure 5.4. BBI image of a U-shaped BVM after stent implantation.....

Figure 5.5. Stent strut after implantation in a U-shaped BVM.

Figure 6.1. Confluent region of cells in a U-shaped BVM cultivated for 1 day...........

Figure 6.2. Histologic cross-section of a dual-sodded U-shaped BVM cultivated for

1 day.

Figure 6.3. BBI image of a dual-sodded U-shaped BVM cultivated for 1 day

Figure 6.4. SEM image of a dual-sodded U-shaped BVM cultivated for 1 day......

Figure 6.5. Histologic cross-section of a dual-sodded U-shaped BVM cultivated for 4 days at a flow rate of $12 \mathrm{~mL} / \mathrm{min}$

Figure 6.6. BBI image of a dual-sodded U-shaped BVM cultivated for 4 days at a flow rate of $12 \mathrm{~mL} / \mathrm{min}$.

Figure 6.7. SEM image of a dual-sodded U-shaped BVM cultivated for 4 days at a flow rate of $12 \mathrm{~mL} / \mathrm{min}$. 133 
Figure 6.8. Histologic cross-section showing identical structures fluorescing green and red.

Figure 6.9. Comparison of HUVEC-only U-shaped BVMs to dual-sodded

U-shaped BVMs

Figure B.1. Locate degraded O-rings

Figure B.2. Wedge razor blade between UV glue and polypropylene container, and start to pry off UV glue.

Figure B.3. Leave razor blade wedged in place, and wedge hemostats between

UV glue and polypropylene container. 158

Figure B.4. Remove razor blade 158

Figure G.1. Microscope anatomy 164

Figure G.2. QCapture Pro control panel. 165

Figure I.1. L-Shaped BVM 1 168

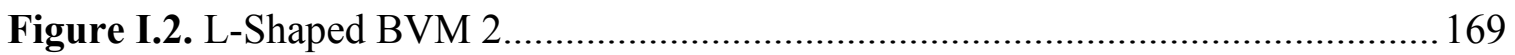

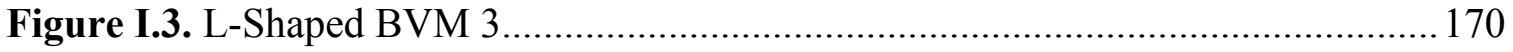

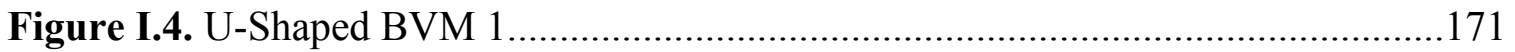

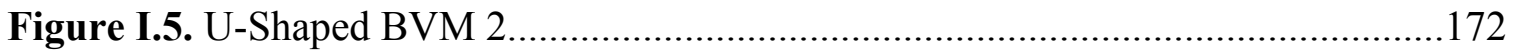

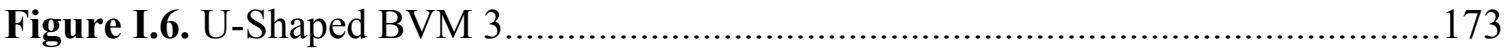

Figure J.1. Minitab output for cell density comparisons throughout L-shaped BVMs. 176

Figure J.1. Minitab output for cell density comparisons throughout U-shaped

BVMs. 177

Figure L.1. Microscope anatomy 180 
Figure L.2. QCapture Pro home window and control panel...................................... 181

Figure L.3. QCapture Pro displays live image of sample........................................... 181

Figure L.4. QCapture Pro adjusts lighting of live image..........................................182 


\section{Introduction}

\subsection{Overview}

The overall goal of this thesis research was to develop a complex-shaped BVM for coronary stent testing.

New coronary devices are constantly under development for the identification and treatment of coronary heart disease (CHD). CHD occurs when plaque occludes coronary arteries, which often requires treatment. Before clinical use of coronary devices, the devices must be tested. Preclinical testing occurs first and involves in vitro laboratory studies and in vivo animal studies. Most in vitro studies do not predict complex biologic responses to three-dimensional coronary devices, and in vivo animal studies are expensive and time-consuming and may show tissue responses that are quite different from human tissue responses. Therefore, tissue engineered blood vessel mimics (BVMs) were recently developed as cost-effective in vitro living models of human blood vessels that can evaluate a human tissue response to three-dimensional coronary devices. BVMs have been developed in only straight geometries, but the FDA has recommended that coronary devices be preclinically tested in complex-shaped simulated vessels when the complex geometries of coronary arteries may negatively affect coronary device performance. Complex geometries of coronary arteries may negatively affect the tissue response to coronary stents, therefore BVMs should be developed in complex geometries.

The rest of this introduction establishes in more detail the reasons that BVMs should be developed in complex geometries. Specifically, this introduction will describe the reasons that the complex geometries of coronary arteries are prone to plaque 
formation, the types of coronary devices that have been used to identify and treat CHD, the preclinical testing methods that have been used to evaluate coronary devices, the FDA recommendations for coronary device testing, the types of synthetic models and animal models that have been used for coronary device testing, the need for BVMs, and the particular need for BVMs in complex geometries.

\subsection{Coronary Heart Disease}

Coronary heart disease (CHD) is the leading cause of death in the United States, and an American dies of CHD almost every minute [1]. 17.6 million Americans currently live with CHD, and costs associated with CHD totaled \$177.1 billion in 2010 [1]. CHD occurs when plaque occludes coronary arteries. Coronary arteries are the blood vessels on the surface of the heart that deliver oxygen- and nutrient-rich blood to the myocardium, which is the cardiac tissue responsible for heart contractions. The occlusion of arteries by plaque is known as atherosclerosis, and atherosclerotic coronary arteries restrict blood flow to the myocardium, starving the myocardium of essential amounts of oxygen and nutrients. This condition impairs the heart's ability to effectively pump blood throughout the body, which causes peripheral tissues to receive insufficient amounts of oxygen and nutrients, resulting in death of those peripheral tissues. Restricted coronary blood flow also causes shortness of breath, myocardial infarction, and death [2]. Because CHD affects such a large population, researchers devote significant attention to developing treatments for CHD. 


\subsection{Anatomy of Coronary Arteries}

When developing treatments for CHD, researchers take into account the complex geometries of coronary arteries (Figure 1.1). The geometries of coronary arteries are considered complex because of bends and bifurcations; a bifurcation is defined as the branching of a main vessel into two smaller vessels [3]. The American College of Cardiology (ACC) and the American Heart Association (AHA) categorize coronary arteries based on the severity of their bends: a "non-angulated" coronary artery bends less than 45 degrees, a "moderately angulated" coronary artery bends 45 degrees to 90 degrees, and an "extremely angulated" coronary artery bends more than 90 degrees [3]. Because coronary arteries are located on the surface of a beating heart, they oscillate between systolic geometries and diastolic geometries: coronary bends become more severe during systole and less severe during diastole [4]. 


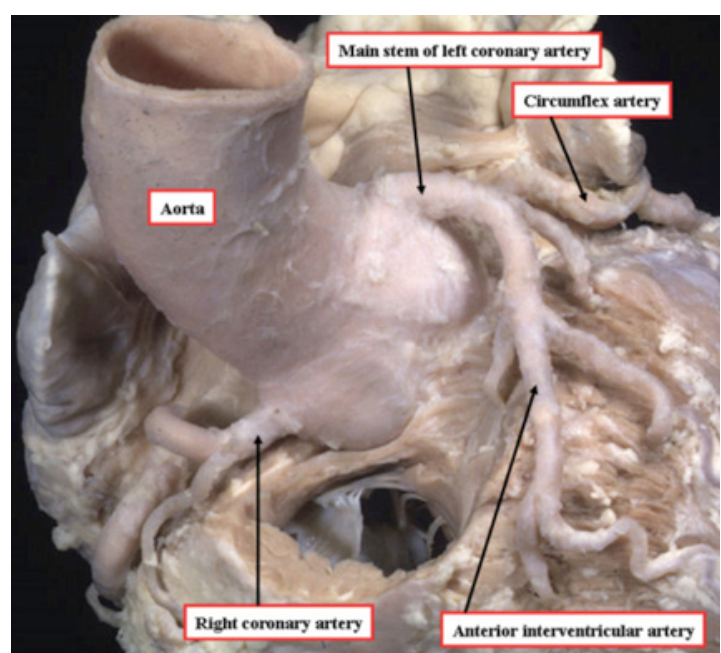

A

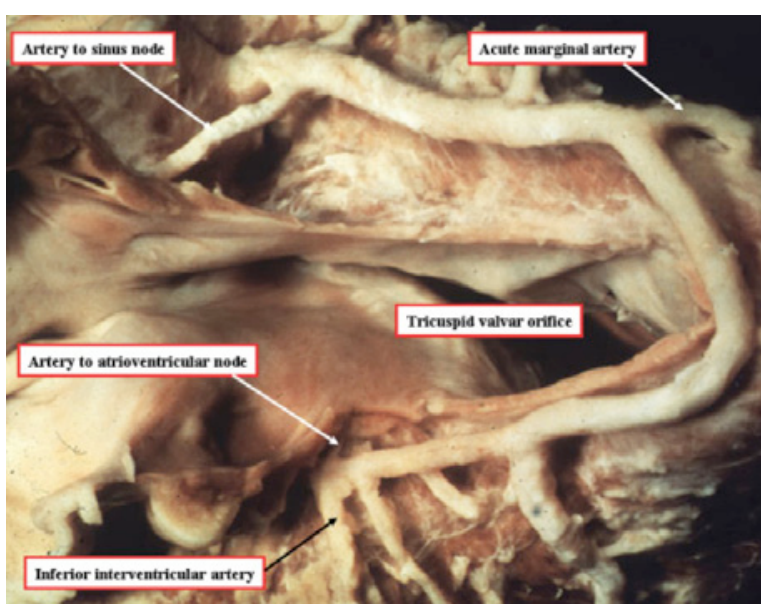

B

Figure 1.1. Anatomy of human coronary arteries. (A) Left and right coronary arteries branch off the aorta from openings known as coronary ostia. The main stem of the left coronary artery bifurcates into the anterior interventricular artery and the circumflex

artery. (B) The right coronary artery gives rise to various branch arteries, including the artery supplying the sinus node, the acute marginal artery, the inferior interventricular artery, and the artery supplying the atrioventricular node. Anatomic variations in coronary arteries between humans do exist but are rare. [5] Reprinted from [5] with permission. See Appendix P.

\subsection{Blood Flow in Coronary Arteries}

Blood flow through the complex geometries of coronary arteries has different characteristics than blood flow through straight arteries. In straight arteries, blood velocity is highest in the center of the artery [6]. In bent coronary arteries, blood velocity is highest near the outer wall of the bend (Figure 1.2 A) [6], and in the branch vessels after a coronary bifurcation, blood velocity is highest near the medial walls (Figure 1.2 B) [6]. Also, blood flow characteristics change as the heart beats: blood flows distally 
through the coronary arteries during diastole as depicted in Figure 1.2 [6], but during systole, blood momentarily flows proximally, opposite the depiction in Figure 1.2 [7]. High pressures during systole cause blood flow to reverse direction in coronary arteries [7].

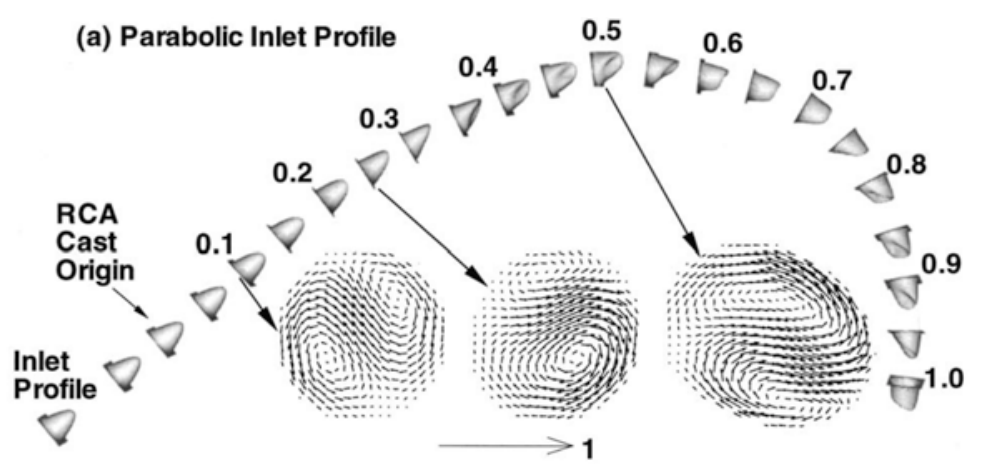

A

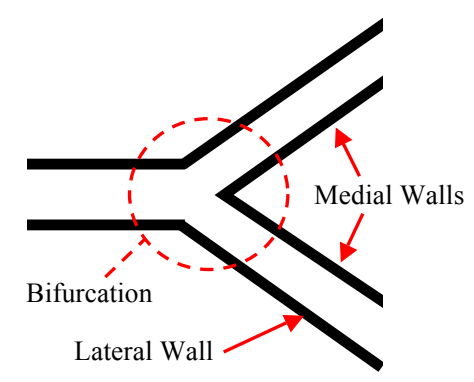

B

Figure 1.2. Schematics of complex-shaped coronary arteries. Blood flows distally (from left to right). The multiple small gray curves in A are velocity profiles. In bent coronary arteries, flow velocity is highest near the outer wall of the bend, so velocity curves are skewed toward the outer wall (A). Branch vessels after a bifurcation have medial walls as identified in B; flow velocity is highest near the medial walls in branch vessels after a bifurcation, so velocity curves would be skewed toward the medial walls. $[6,8]$ Figure 1.2 A reprinted from [8] with permission. See Appendix P.

\subsection{Shear Stress and Atherosclerosis in Coronary Arteries}

The unique blood flow that occurs in complex-shaped coronary arteries creates unique shear stress distributions on the coronary walls. In bent coronary arteries, blood flows faster near the outer wall of the bend and slower near the inner wall of the bend; these blood flow characteristics create low shear stress on the inner walls of bent 
coronary arteries [6]. In the branch vessels after a bifurcation, blood flows faster near the medial walls and slower near the lateral walls; these blood flow characteristics create low shear stress on the lateral walls of branch vessels after a bifurcation [6]. Also, blood flow that changes direction as the heart cycles through diastole and systole is called oscillatory blood flow and creates oscillatory shear stress on coronary walls [9].

Low and oscillatory shear stresses are associated with atherosclerosis $[9,10]$. A low endothelial shear stress environment is the initiating process of atherosclerosis in an atherosclerosis-prone host and typically occurs on the inner walls of curved arteries [9]. Oscillatory shear stress is associated with early and continued atherosclerosis and typically occurs on the lateral walls of branch vessels after a bifurcation $[9,10]$. While normal shear stress magnitudes are more than $15 \mathrm{dynes} / \mathrm{cm}^{2}$ [11], low shear stress magnitudes are less than $10-12$ dynes $/ \mathrm{cm}^{2}[9]$.

Endothelial cells line the lumen of coronary arteries, and low shear stress as a result of the unique blood flow that occurs in complex-shaped coronary arteries directly affects endothelial cells [12]. Endothelial cells exposed to low shear stress acquire an atherogenic phenotype and encourage formation of early atherosclerotic plaques by promoting lipid accumulation [9]. Eventually lipids accumulate in the inner lining of arteries - the intima — and form large lipid pools that cause cell necrosis [13]. Fibrous tissue then encapsulates the lipids and necrotic cells, and the encapsulation is known as an early fibroatheroma, the initial plaque that can develop into more lethal plaques [13]. The kind of plaque that will develop from an early fibroatheroma depends on local factors (e.g. the magnitude of shear stress on endothelial cells), systemic factors, and genetic factors. There are three kinds of plaques that can develop from an early 
fibroatheroma: quiescent plaques, stenotic plaques, and thin-cap fibroatheromas (TCFAs)

(Figure 1.3) [9]. Quiescent plaques cause no symptoms in patients, stenotic plaques restrict blood flow, and TCFAs may rupture and cause thrombosis $[9,13]$.

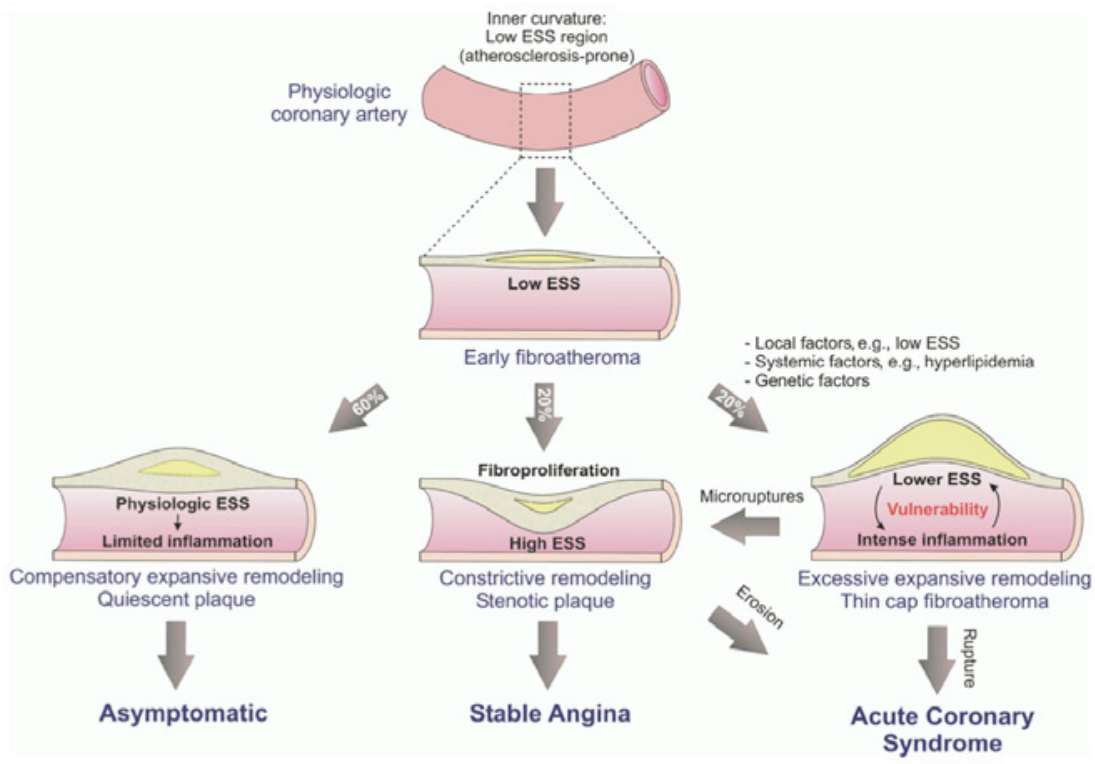

Figure 1.3. Plaque development in bent coronary arteries. The inner wall of bent coronary arteries experiences low endothelial shear stress (ESS), causing formation of early fibroatheromas. Arteries may then undergo compensatory expansive remodeling that causes quiescent plaque formation, constrictive remodeling that causes stenotic plaque formation, or excessive expansive remodeling that causes TCFA formation. [9] Reprinted from [9] with permission. See Appendix P.

Plaques categorized as stenotic plaques and as TCFAs that ruptured and caused thrombosis are alike in that they can eventually occlude coronary arteries, which prevents healthy blood flow to the myocardium and leads to CHD. Because approximately $40 \%$ of early fibroatheromas develop into stenotic plaques and TCFAs [9], and because both 
plaques can eventually cause coronary occlusion, researchers aiming to identify and treat CHD develop devices specifically for identifying and treating coronary occlusions.

\subsection{Devices for Identifying and Treating Coronary Heart Disease}

Patients must first receive a diagnosis of CHD before potential treatment for it can begin. When diagnosing patients, physicians use various imaging technologies that help visualize narrowed coronary arteries and plaque formations. After compromised coronary arteries are identified, various treatments may be used to treat coronary occlusions.

\subsubsection{Identifying Coronary Heart Disease}

Angiography is the standard imaging technique used to identify obstructed coronary arteries [14]. Angiography involves the insertion of a diagnostic catheter into the coronary ostia - the openings of the coronary arteries on the aorta-followed by injection of a radiopaque dye into the coronary arteries, followed by acquisition of an $\mathrm{x}$ ray image known as an angiogram (Figure 1.4) [15]. 


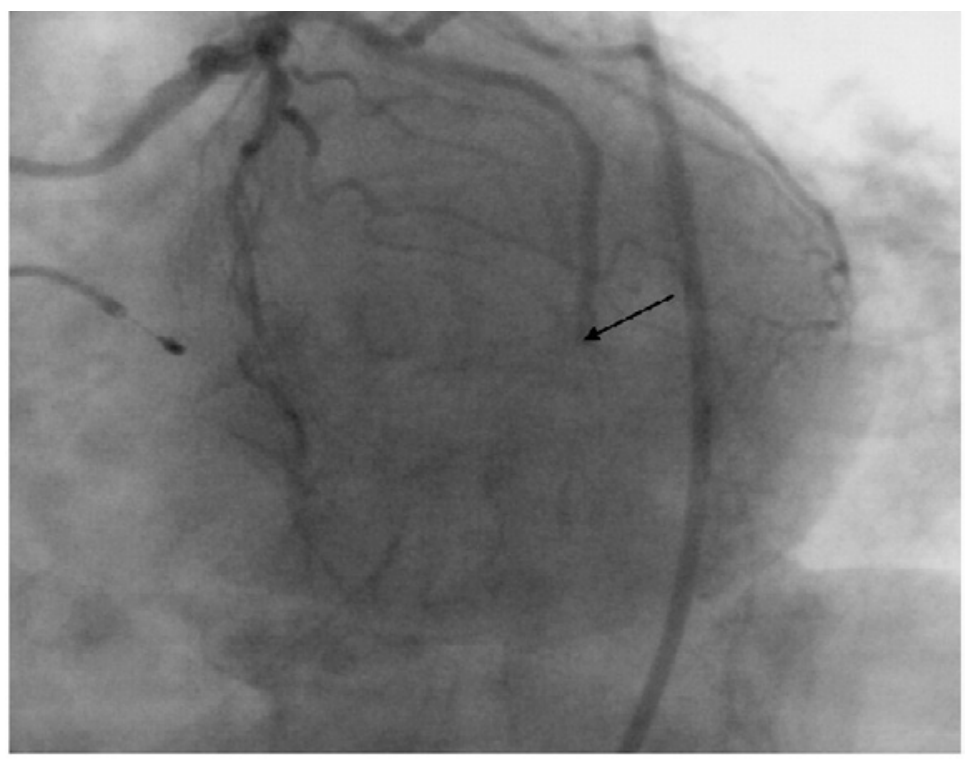

Figure 1.4. Angiogram of coronary arteries. Coronary lumens appear as dark lines, and the black arrow points to a site of total occlusion in a left circumflex coronary artery. [16] Reprinted from [16] with permission. See Appendix $P$.

Although angiography is the standard imaging technique used to identify obstructed coronary arteries, intravascular ultrasound (IVUS) has the advantage of revealing more details about the severity and extent of atherosclerosis [17]. IVUS systems generate intravascular images and consist of a catheter, a miniature transducer on the catheter, and a console that processes electrical signals and displays the intravascular images [17]. To obtain an intravascular image of a coronary artery, an IVUS catheter is inserted into the lumen of the coronary artery and pushed through the lumen until the catheter tip reaches regions that require assessment. Ultrasound waves are then reflected off the walls of the artery, and the reflected waves are converted to electrical signals and processed to generate an image. 
Angiography, IVUS, and other imaging technologies each have advantages when used individually to visualize compromised coronary arteries, but researchers are currently combining imaging technologies to create hybrid imaging systems. Such hybrid systems combine the advantages of individual imaging technologies to generate extremely useful information. For example, a system that combines IVUS and near infrared spectroscopy (NIRS) allows specific plaque characteristics in coronary arteries to be identified, which may allow researchers to study changes in those specific plaque characteristics in response to new treatments [17]. Researchers then may be able to better understand the exact effects of the new treatments and then adjust the new treatments to work more effectively.

\subsubsection{Angioplasty}

After a physician identifies blockages in a patient's coronary arteries, the patient typically receives some sort of intervention that treats the blockages and restores blood flow through the blockage site. An established treatment for coronary blockages is percutaneous transluminal coronary angioplasty (PTCA) [18]. PTCA is a minimally invasive procedure that involves compression of plaque against the coronary walls. The following steps are carried out during a PTCA procedure in the clinic: injection of local anesthesia into the groin area; placement of a needle into the femoral artery; placement of a guide wire through the needle into the femoral artery; movement of the guide wire proximally through the femoral artery and eventually to the aorta; placement of a diagnostic catheter over the guide wire and movement of the diagnostic catheter to the aorta; removal of the guide wire; placement of the diagnostic catheter into an ostium on 
the aorta; injection of contrast media into the coronary arteries; acquisition of an angiogram; identification of a treatable blockage in the angiogram; placement of a guide wire through the blockage site in the coronary arteries; placement, inflation, deflation, and removal of a balloon at the blockage site (Figure 1.5); use of the balloon at other blockage sites in the coronary arteries; injection of contrast media and acquisition of an angiogram to check for improvements in luminal diameters; and removal of the catheter [15].

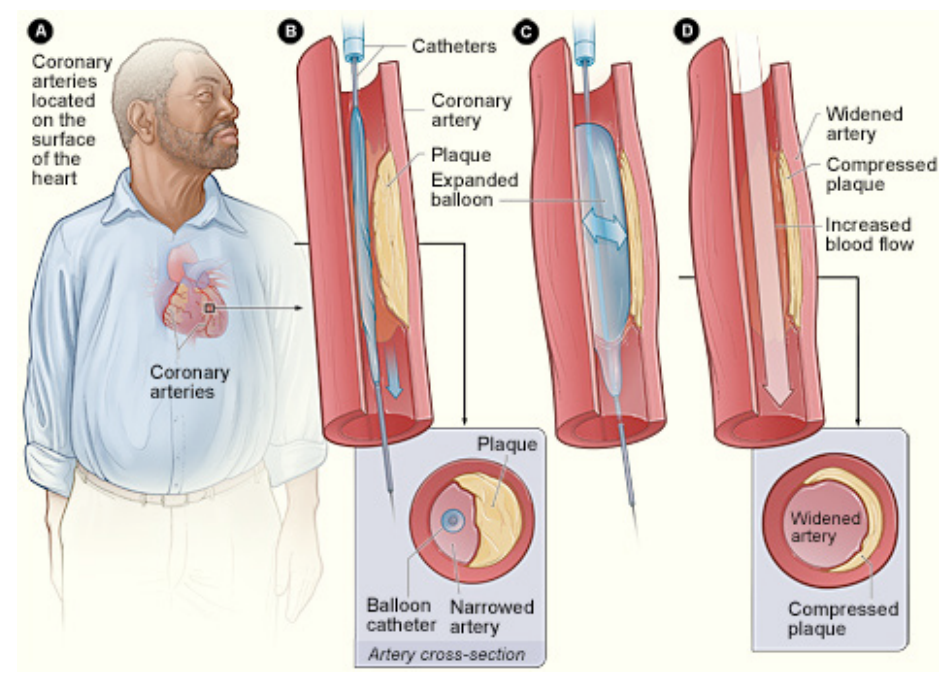

Figure 1.5. Placement, inflation, deflation, and removal of a balloon during PTCA. A guide wire is placed across the blockage site in a coronary artery, then a balloon catheter is placed at the blockage site (B). A balloon is inflated (C) to widen the lumen and then deflated. The balloon is repeatedly inflated and deflated with increasing amounts of pressure during inflation to further widen the lumen and is then removed, leaving behind a widened coronary artery (D). [19] Reprinted from [19] with permission. See Appendix P. 
Although PTCA is an established treatment for coronary occlusions, there is a notable drawback to PTCA: restenosis [20]. Restenosis is the re-narrowing of a blood vessel after treatment. Restenosis after PTCA can occur as a result of early elastic recoil, thrombus formation, neointimal formation, and vascular remodeling [21-23]. Early elastic recoil occurs when a blood vessel that was widened via PTCA recoils within minutes to hours after the procedure $[24,25]$. Thrombus formation occurs when blood clots at the treatment site [26]; this event is also known as thrombosis. Neointimal formation occurs after traumatic balloon injury and involves the migration of proliferating vascular smooth muscle cells to the intima to form new tissue [27]. Vascular remodeling occurs when the structure of a blood vessel is altered as a result of changes in cell growth, cell death, cell migration, and production or degradation of extracellular matrix [28]. To eliminate restenosis after PTCA, researchers are currently developing new PTCA technology.

One technology currently under development is balloons that deliver drugs to the walls of coronary arteries to reduce neointimal formation, thereby reducing restenosis. When designing such balloons, researchers must choose drugs that best reduce neointimal formation. Some new balloons deliver paclitaxel, which inhibits smooth muscle cell proliferation and migration [29]. In addition to choosing the best drugs, researchers must also choose design features that deliver adequate quantities of drugs, minimize injury to the coronary wall, do not limit blood flow through the treated segment of blood vessel, and administer drugs for sufficient time to cause a therapeutic effect [18]. An example of a drug-delivering balloon is found on the $\mathrm{GENIE}^{\mathrm{TM}}$ balloon catheter: the balloon has proximal and distal occlusive segments to restrict drug delivery to the space between the occlusive segments, and liquid paclitaxel jets out of the balloon onto the coronary walls 
[18]. Another example of a drug-delivering balloon is found on the Sequent ${ }^{\circledR}$ Please balloon catheter: the balloon is homogenously coated with paclitaxel that is embedded in contrast medium; when the balloon is inflated, the contrast medium releases the paclitaxel into the surrounding coronary artery [18]. More research is being conducted to further identify the best features for drug-delivering balloons that treat restenosis.

\subsubsection{Stents}

Another technology for treating coronary occlusions is stents [30]. Stents are latticed tubes that can be crimped onto catheters, placed into the body in a minimally invasive way, and then expanded once inside an occluded region of a blood vessel to prop open the occluded region. Most commercially available coronary stent systems feature balloon-expandable stents [21], but stents are also made in self-expanding versions. Balloon-expandable stents can be deployed either after PTCA or simply without PTCA, whereas self-expanding stents are designed for deployment after PTCA [21, 31]. The following steps may be carried out during a stenting procedure in the clinic: the PTCA procedure described previously; placement of a balloon catheter with a crimped stent into the blockage site; deployment of the stent at the blockage site (Figure 1.6); deployment of stents at other blockage sites throughout the coronary arteries; and injection of contrast media and acquisition of an angiogram to check for improvements in luminal diameters [15]. Stenting is currently the principal method of percutaneous coronary revascularization for patients with $\mathrm{CHD}[32]$. 


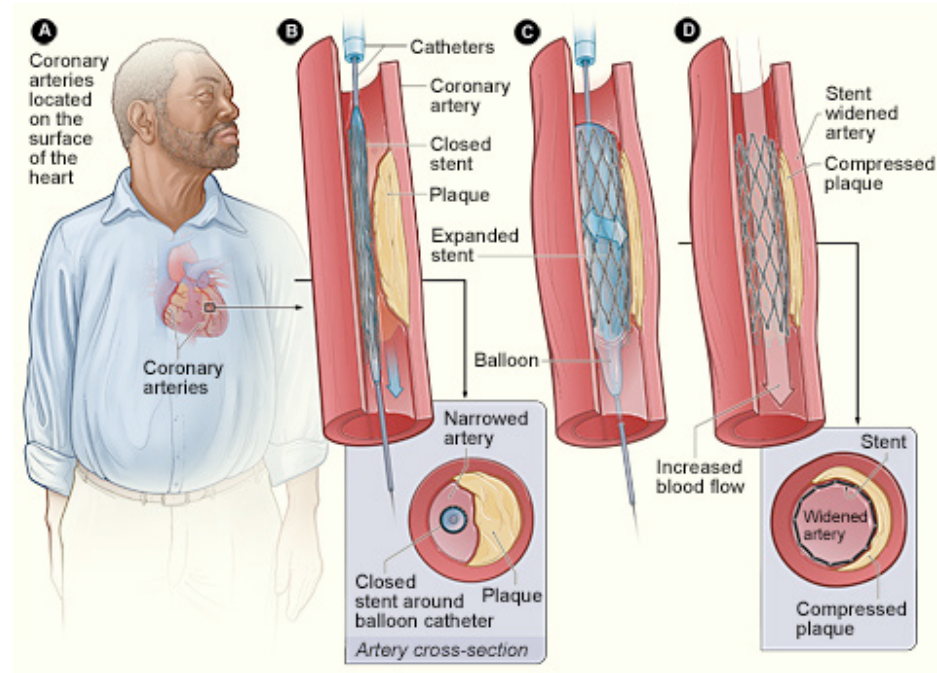

Figure 1.6. Deployment of a balloon-expandable stent in a coronary artery. A balloon catheter with a closed stent is inserted into the blockage site (B), the balloon is inflated to deploy the stent $(\mathrm{C})$, and the balloon is deflated and removed along with the catheter and guide wire, leaving behind the implanted stent, the compressed plaque, and the widened coronary artery (D). The stent lattice is made up of segments known as stent struts. [15] Reprinted from [15] with permission. See Appendix P.

Although stenting is currently a principal treatment for CHD, there are notable drawbacks to stenting. The first stents developed were bare metal stents (BMSs) and were intended to reduce the restenosis that occurs after PTCA. BMSs prevent elastic recoil of blood vessels and limit negative remodeling of blood vessels [21, 33], but BMSs do not prevent neointimal formation. Blood vessels treated with BMSs may still experience neointimal formation, and neointimal formation in the stented region of a blood vessel causes in-stent restenosis [33]. To ameliorate in-stent restenosis, drug-eluting stents (DESs) were developed. DESs are metal latticed tubes coated with an agent that prevents cell proliferation. Because the proliferation of vascular smooth muscle cells in the intima 
causes neointimal formation, blood vessels treated with DESs experience reduced neointimal formation [18]. But blood vessels treated with DESs may also experience thrombosis. Therefore, although BMSs and DESs both improve upon previous technology, they also both have disadvantages. Because of these disadvantages, researchers continue improving stent technology.

New stent technology currently under development is the dual drug-eluting stent (DDES). Since blood vessels treated with DESs may experience thrombosis, DDESs were developed as an improved version of DESs: like DESs, DDESs contain an agent that prevents cell proliferation, but unlike DESs, DDESs also contain an agent that prevents thrombosis [34]. Studies on DDESs are promising: one study showed that a DDES containing sirolimus for treating restenosis and triflusal for treating thrombosis significantly reduced restenosis compared to BMSs and DESs when implanted in a pig for 30 days, and the reduction in restenosis was associated with an inhibition of inflammation and thrombus formation [34]; another study showed that a DDES containing sirolimus and probucol required less revascularization compared to DESs when implanted in humans for two years [35]. Therefore, DDESs may be an improvement over previous stent technology.

Another improvement over previous stent technology may be the bioabsorbable stent. Bioabsorbable stents prop open occluded blood vessels for a defined period of time, ideally completely dissolve after the defined period of time, and ideally leave behind a fully healed natural blood vessel [18]. An advantage of bioabsorbable stents is the restoration of natural vascular remodeling after stent dissolution: after deployment of BMSs, DESs, and DDESs, vascular remodeling freezes in the stented region of the blood 
vessel, but after deployment and dissolution of a bioabsorbable stent, natural vascular remodeling may be restored [36]. Another advantage of bioabsorbable stents is the minimization of late stent thrombosis: after deployment of DESs, late stent thrombosis can occur, but after deployment of a bioabsorbable stent, the bioabsorbable stent may dissolve before late stent thrombosis occurs and may therefore avoid late stent thrombosis altogether [18]. Studies on bioabsorbable stents are promising: one study showed complete dissolution of a bioabsorbable stent deployed in human coronary arteries for two years [37]. Because bioabsorbable devices are currently considered a revolutionary technology in the field of interventional cardiology [38], researchers continue improving bioabsorbable stents.

\subsection{Coronary Device Testing}

There are a number of coronary devices currently under development for the identification and treatment of CHD, including hybrid intravascular imaging systems, drug-delivering balloons, DDESs, and bioabsorbable stents. But the Food and Drug Administration (FDA) requires that these coronary devices be tested before they are marketed in the United States. The FDA approves a coronary device for market only after technical data from non-clinical and clinical studies show that the coronary device is safe and effective. Non-clinical studies, also known as preclinical studies, refer to in vitro laboratory studies and in vivo animal studies, and clinical studies refer to in vivo human studies. To help researchers carry out coronary device studies that are well designed in the eyes of the FDA, the FDA publishes recommendations for the design of coronary device studies [39]. FDA recommendations emphasize that in various cases coronary 
devices should be preclinically tested specifically under the conditions that exist in complex-shaped vessels.

\subsubsection{FDA Recommendations for In Vitro Studies}

FDA recommendations for various in vitro engineering studies emphasize that coronary devices should be tested in complex-shaped simulated vessels [39, 40]. For some in vitro studies, the FDA recommends that a coronary stent intended for deployment in a coronary bend be tested in a simulated vessel that is bent to a worst-case clinically relevant radius of curvature; the FDA usually recommends a 15-millimeter radius of curvature because this represents the worst-case radius of curvature in $90 \%$ of patients [40]. For other in vitro studies, the FDA recommends that a coronary stent intended for deployment in a coronary bifurcation be tested in a simulated vessel that has a geometry based on relevant clinical situations [40]. For other in vitro studies, the FDA recommends that coronary delivery catheters be tested in simulated vessels that are tortuous, and the specific degree of tortuosity is shown in Figure X2.4 of ASTM F2394 [40].

\subsubsection{Synthetic Models of Coronary Vasculature}

Researchers seeking FDA approval of their coronary devices test their devices in complex-shaped simulated vessels. Complex-shaped simulated vessels range from simple tubes that can be bent into desired geometries to physiologically accurate replications of coronary vasculature [41-43]. One example of a complex-shaped simulated vessel is found on the Interventional Device Testing Equipment 2000 (IDTE2000) made by 
Machine Solutions, Inc.; the IDTE2000 features simple tubing that can be bent to model complex geometries of coronary arteries (Figure 1.7 A and B) [41]. Another example of a complex-shaped simulated vessel is the Angio-Suite Training Model produced by United Biologics, Inc.; the Angio-Suite Training model is a physiologically accurate replication of many arteries in the human body, including major coronary arteries (Figure 1.8) [43]. The simple tubing of the IDTE2000, the physiologically accurate Angio-Suite Training Model, and other complex-shaped simulated vessels may be used to assess various aspects of coronary devices.

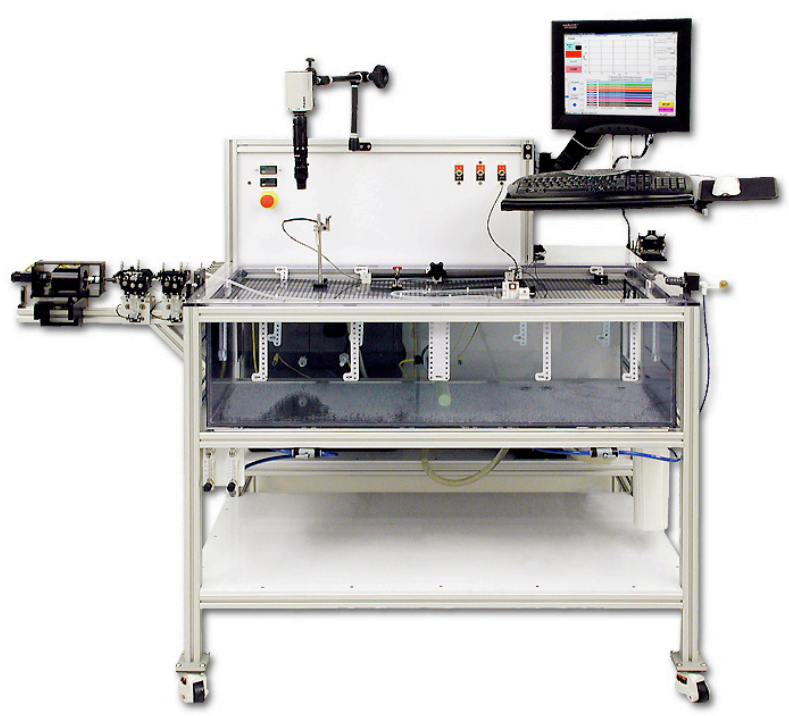

A

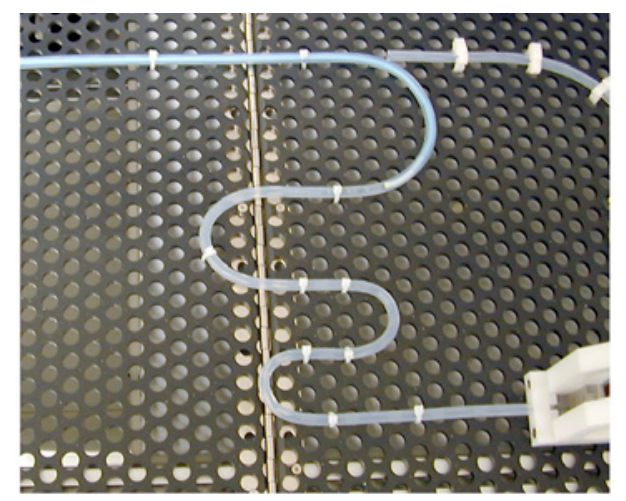

B

Figure 1.7. A complex-shaped simulated vessel. The IDTE2000 by Machine Solutions, Inc. (A) features simple tubing that can be bent into complex geometries (B). [41] Reprinted from [41] with permission. See Appendix $P$. 


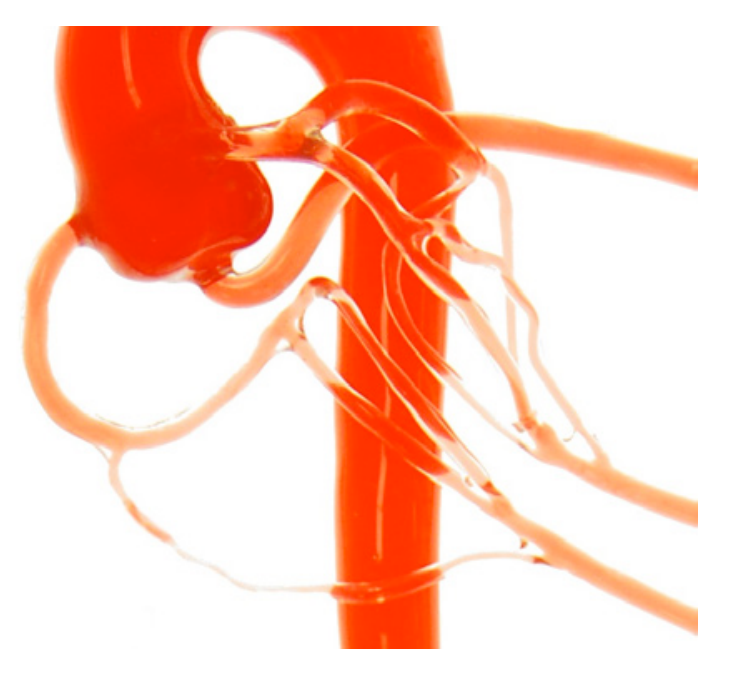

Figure 1.8. A physiologically accurate complex-shaped simulated vessel. The AngioSuite Training Model by United Biologics, Inc. includes silicone coronary arteries. [43] Reprinted from [43] with permission. See Appendix $P$.

Aspects of coronary devices that may be assessed in complex-shaped simulated vessels include the following: the ability of a coronary stent to conform to a complexshaped vessel after the stent is deployed, also known as flexibility [44]; the ability of a coronary stent and delivery system to bend when negotiating a complex-shaped vessel, also known as flexibility [44]; the uniformity of a stent after deployment [44]; the ability of a balloon to be deflated and withdrawn from a deployed stent, also known as balloon deflatability [44]; the force needed to move a coronary device through a tortuous path, also known as track force [41]; the ability of a catheter tip to track over a bent guidewire, also known as trackability [44]; the force needed to withdraw a coronary device from a tortuous path, also known as retractability force [41]; the coating integrity of a coronary DES after deployment of the DES in a complex-shaped vessel [39]; the formation of particulate matter during tracking and deployment of a coronary DES in a complex- 
shaped vessel [39]; and image distortion that may occur when intravascular imaging devices negotiate bent vessels [45].

Although these complex-shaped simulated vessels provide researchers with valuable information about coronary device performance, these bench testing systems do not predict biologic responses that may occur when using coronary devices in human patients. One way to predict some biologic responses is to expose coronary devices or parts of devices to biologic samples in a laboratory. But most in vitro laboratory tests cannot predict complex biologic responses such as the human tissue response to a threedimensional coronary stent.

\subsubsection{In Vivo Animal Models}

After coronary devices undergo and "pass" in vitro tests in complex-shaped simulated vessels and in vitro tests involving exposure to biologic samples, in vivo animal testing is used to predict complex biologic responses that may occur when using coronary devices in human patients. Various animals may be used to model complex biologic responses. Rats have carotid arteries and an abdominal aorta that may be used to assess thrombus formation and neointimal responses after stent implantation [46, 47]; also, rats may be useful for assessing proliferative responses [48]. Rabbits have iliac arteries that may be used to assess the mechanisms of repair after injury to an abnormal artery [48]. Porcine models have a coronary artery system that is similar in size and structure to humans and that can be used to assess events after stent implantation such as injury, neointimal development, and thrombosis $[49,50]$. Porcine models are currently the closest to humans [48]. 
An ideal animal model would perfectly predict the following human tissue responses. First, during coronary stent deployment, the stent immediately contacts the endothelium and removes endothelial cells from the vessel wall, exposing smooth muscle cells beneath the endothelium [51,52]; this event is known as endothelial denudation. Immediately after stent deployment, the stent is susceptible to thrombosis for an acute period. Acute inflammation then occurs, and inflammatory cells reach the stented site. Migration and proliferation of vascular smooth muscle cells begin [53], and vascular smooth muscle cells synthesize extracellular matrix proteins and eventually cause neointimal formation. Finally, vascular remodeling occurs, and endothelial cells grow over the entire inner surface of the stented region of blood vessel [51]. This endothelial cell growth is known as re-endothelialization and is key to successful healing after stent implantation. A confluent monolayer of endothelial cells modulates local hemostasis and thrombolysis, protects vascular smooth muscle cells from circulating growth-promoting factors, synthesizes growth factors that are important in vascular smooth muscle cell proliferation, and plays a role in the quiescence of smooth muscle cells [52]. Although researchers use animal models to predict these human tissue responses, animal tissue responses can vary from human tissue responses (Figure 1.9 A and C) [51, 54]. 

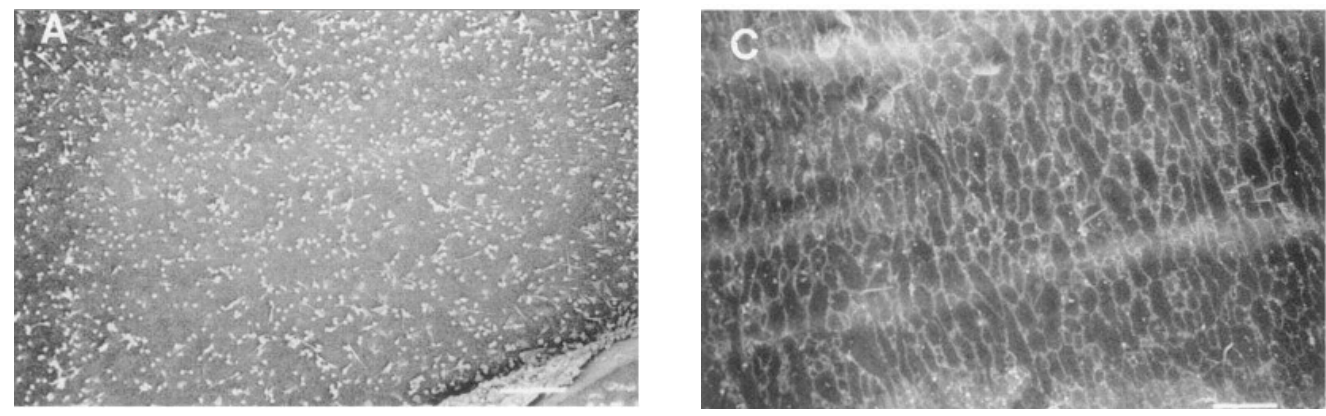

Figure 1.9. Re-endothelialization of a stent in a rabbit iliac artery. On the day of stent implantation, blood cells but not endothelial cells are visible on the stent (A). 28 days after stenting, a confluent monolayer of endothelial cells has formed, and reendothelialization of the stent is complete (C). In human coronary arteries, complete reendothelialization of coronary stents has been observed 96 days after stenting. [51, 54] Reprinted from [54] with permission. See Appendix $P$.

Although animal models are widely used to assess complex biologic responses to coronary devices, there are notable drawbacks. Sometimes the tissue response of all available animal models can be quite different from the human tissue response $[48,55$, 56]. Also, studies involving animal models are expensive and time-consuming. There are currently many coronary devices under development that require testing-hybrid intravascular imaging systems, drug-delivering balloons, DDESs, and bioabsorbable stents are just a few examples described previously — and possibly many prototypes of these coronary devices will first be developed, each prototype requiring animal testing to ensure that the best prototype is selected. But it is not feasible to test all coronary device prototypes in animals because of the expense and time consumption of animal testing, therefore some prototypes with outstanding in vivo performance may be overlooked. To improve in vivo animal testing, these drawbacks should be ameliorated. 


\subsubsection{Blood Vessel Mimics}

There are a number of drawbacks to all the preclinical testing systems described so far. Bench tests involving complex-shaped simulated vessels do not predict biologic responses that may occur when using coronary devices in human patients. Most in vitro tests involving exposure of coronary devices or parts of devices to biologic samples do not predict some complex biologic responses such as the human tissue response to a three-dimensional coronary stent. Animal models may exhibit tissue responses that are quite different from human tissue responses, and studies involving animal models are expensive and time-consuming. These drawbacks prevent the best possible preclinical assessments of coronary devices, therefore researchers aim to develop preclinical testing systems that ameliorate these drawbacks.

A preclinical testing system was recently developed that ameliorates some of the previously described drawbacks: the blood vessel mimic (BVM). A BVM is a tissue engineered blood vessel, consisting of a tubular scaffold that has human endothelial cells lining the inner surface. Up until now, BVMs have been developed in straight geometries. BVMs have been used to specifically test the re-endothelialization of stents: stents have been deployed in BVMs, cells from the BVMs have re-endothelialized the stents, and BVMs have identified stents that exhibit better re-endothelialization than other stents. Unlike the in vitro testing methods described previously, BVMs feature the ability to evaluate re-endothelialization of a three-dimensional stent. Unlike the in vivo animal models described previously, BVMs feature a human tissue response, cost-effectiveness, and high-throughput screening. [57-59] 


\subsection{Complex-Shaped Blood Vessel Mimics}

Although BVMs do ameliorate some of the previously described drawbacks to preclinical testing systems, these mimics could still be modified to better align with specific FDA recommendations. As described, the FDA has recommended that in various cases coronary devices be preclinically tested in simulated vessels that mimic the complex geometries of coronary arteries [40]. The FDA has made this recommendation when the complex geometries of coronary arteries create conditions that can negatively affect coronary device performance. If the complex geometries of coronary arteries negatively affect re-endothelialization of coronary stents, the FDA may recommend that BVMs be developed in complex geometries to more accurately assess the unique reendothelialization that occurs.

The complex geometries of coronary arteries may in fact negatively affect reendothelialization of coronary stents. When a coronary stent is deployed in a coronary bend, the stent may be more rigid than the artery and straighten out the bend [60]. This straightening skews the blood velocity profile at the bend and creates regions of decreased endothelial shear stress $[61,62]$. In regions of low shear stress, the endothelium re-establishes itself more slowly [63]. If there are large regions where the endothelium has not re-established itself, these large areas of endothelial denudation are associated with intimal thickening [64]. Intimal thickening is the cause of in-stent restenosis, and coronary arteries that have been straightened with stents are associated with in-stent restenosis and major adverse clinical events [60]. Also, when a coronary stent is deployed in a coronary bifurcation, stent struts may remain suspended over the ostia of branch vessels, not contacting any part of the vessel wall. Stent struts suspended 
over coronary ostia have been shown to not re-endothelialize (Figure 1.10), and stents deployed in coronary bifurcations have been associated with late stent thrombosis [65]. Therefore, bent and bifurcated coronary geometries may negatively affect reendothelialization of coronary stents and may lead to adverse events after stent implantation.

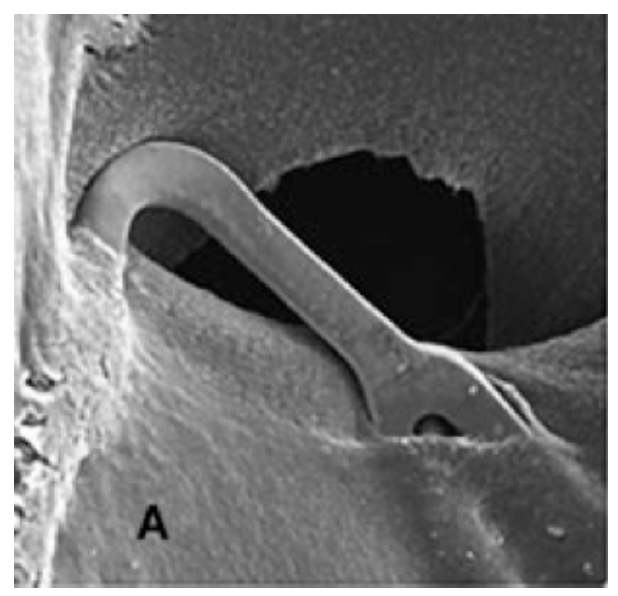

Figure 1.10. Re-endothelialization of a stent in a coronary bifurcation. Coronary stents were implanted in porcine coronary bifurcations. Some stent struts that were suspended over coronary ostia exhibited no re-endothelialization 7 days after implantation. [65] Reprinted from [65] with permission. See Appendix $P$.

Because the complex geometries of coronary arteries may in fact negatively affect re-endothelialization of coronary stents, the currently straight BVMs should be redeveloped in complex geometries for coronary stent testing. Complex-shaped BVMs may more accurately assess the re-endothelialization of coronary stents and may better align with FDA recommendations. 


\subsection{Summary and Aims of This Thesis}

Due to the prevalence of coronary heart disease, many new coronary devices are being developed. The FDA requires that these coronary devices be tested before they are marketed in the United States, and preclinical tests are carried out first. It is advantageous to have preclinical testing methods that are high-throughput and cost-effective, that assess human tissue responses to three-dimensional coronary devices, and that take into account other conditions such as coronary geometry. Current BVMs have many of these characteristics but do not mimic the complex geometries of coronary arteries. Therefore, complex-shaped BVMs should be developed for coronary stent testing.

There are multiple potential challenges that could hinder development of a complex-shaped BVM. First, complex-shaped scaffolds and bioreactors that can house complex-shaped scaffolds have not been fully developed. Second, the technique currently used to deposit cells onto a straight scaffold may result in no cell deposition in certain regions of complex-shaped scaffolds. Third, when fluid is flowing through scaffolds, an extremely angulated scaffold exhibits more than 7 times the wall shear stress of a straight scaffold [66]; higher wall shear stress in a complex-shaped scaffold may reduce cell retention on the scaffold, possibly hindering formation of any confluent cell lining throughout the scaffold. Fourth, some current straight BVMs have been developed using human umbilical vein endothelial cells (HUVECs), which are known to only grow in monolayers [67], and coronary stents have been shown to completely denude the endothelium — which is a monolayer - in human coronary arteries [51, 68]; therefore, stent deployment may completely denude a monolayer of HUVECs in a complex-shaped BVM. If a complex-shaped BVM that was developed using only HUVECs exhibits 
denudation after stent deployment, it may be advantageous instead to use cell types that are known to form multiple cell layers so that the cells could eventually be cultivated into a multilayer that would withstand stent deployment better than a monolayer. Research in the following chapters addresses all these potential challenges.

The goal of this thesis research was to develop a complex-shaped BVM for coronary stent testing. To achieve this goal, the following overall hypothesis was established: a BVM could be developed in the complex geometries of coronary arteries. This hypothesis was tested by conducting research with the following five specific aims.

1. Design, construct, and validate complex-shaped scaffolds and bioreactors.

a. Hypothesis: complex-shaped scaffolds could be designed and constructed, bioreactors capable of housing the new scaffolds could be designed and constructed, and cells would deposit onto the new scaffolds inside the new bioreactors.

2. Deposit cells throughout the entire inner surface of complex-shaped scaffolds.

a. Hypothesis: after 1 day of cultivation of complex-shaped BVMs, no statistically significant differences would exist between the cell densities along the length of the BVMs, between the cell densities in the inner and outer halves of the BVMs, or between the cell densities in the top and bottom halves of the BVMs.

3. Determine whether HUVECs can reach confluence on a complex-shaped scaffold using a cultivation protocol similar to the straight-BVM cultivation protocol.

a. Hypothesis: after deposition of HUVECs onto U-shaped scaffolds and cultivation similar to the straight-BVM protocol, cells would reach confluence. 
4. Determine whether a complex-shaped BVM that was developed using only HUVECs would exhibit denudation of its cell lining after coronary stent deployment.

a. Hypothesis: a complex-shaped BVM that was developed using only HUVECs would exhibit no cell lining after coronary stent deployment.

5. Develop a confluent region of cells in a U-shaped BVM.

a. Hypothesis: a confluent region of cells would form in a U-shaped BVM. If research addressing the fourth specific aim showed that a complex-shaped BVM that was developed using HUVECs may exhibit denudation of its cell lining after coronary stent deployment, the confluent region of cells had to be developed using a cell type known to form multiple cell layers so that the cells could eventually be cultivated into a multilayer that could withstand stent deployment better than a HUVEC monolayer.

Experiments were conducted to test all these hypotheses, and findings from these experiments are described in the following chapters. Chapter 2 describes the design, construction, and validation of complex-shaped scaffolds and bioreactors that can house complex-shaped scaffolds. Chapter 3 describes the consistency of cell deposition throughout various regions of complex-shaped scaffolds. Chapter 4 describes the effect of flow rate on cell confluence in complex-shaped BVMs. Chapter 5 describes stent deployment in a complex-shaped BVM. Chapter 6 describes the development of a confluent region of cells on an extremely angulated scaffold. And chapter 7 concludes this research with a summary and discussion. 


\section{Development of Complex-Shaped Scaffolds and Bioreactors}

\subsection{Introduction}

This chapter addresses the first specific aim of this thesis: design, construct, and validate complex-shaped scaffolds and bioreactors that can house complex-shaped scaffolds.

Complex-shaped BVMs will require new scaffolds and new bioreactors. A bioreactor is a device that allows conditions to be closely controlled to induce specific behavior in the cells or tissues being cultivated $[69,70]$. A BVM is created by first inserting a scaffold into a bioreactor, then depositing human endothelial cells onto the scaffold, and then cultivating the cell-lined scaffold in the bioreactor (Figures 2.1 and 2.2). Current BVMs have been developed using only straight scaffolds, and the bioreactor currently used to cultivate BVMs is capable of housing only these straight scaffolds [71]. Complex-shaped scaffolds and bioreactors that can house complex-shaped scaffolds have not been fully developed. Therefore, they must be fully designed, constructed, and validated. The hypothesis in this chapter was that complex-shaped scaffolds could be designed and constructed, that bioreactors capable of housing the new scaffolds could be designed and constructed, and that cells would deposit onto the new scaffolds inside the new bioreactors. 


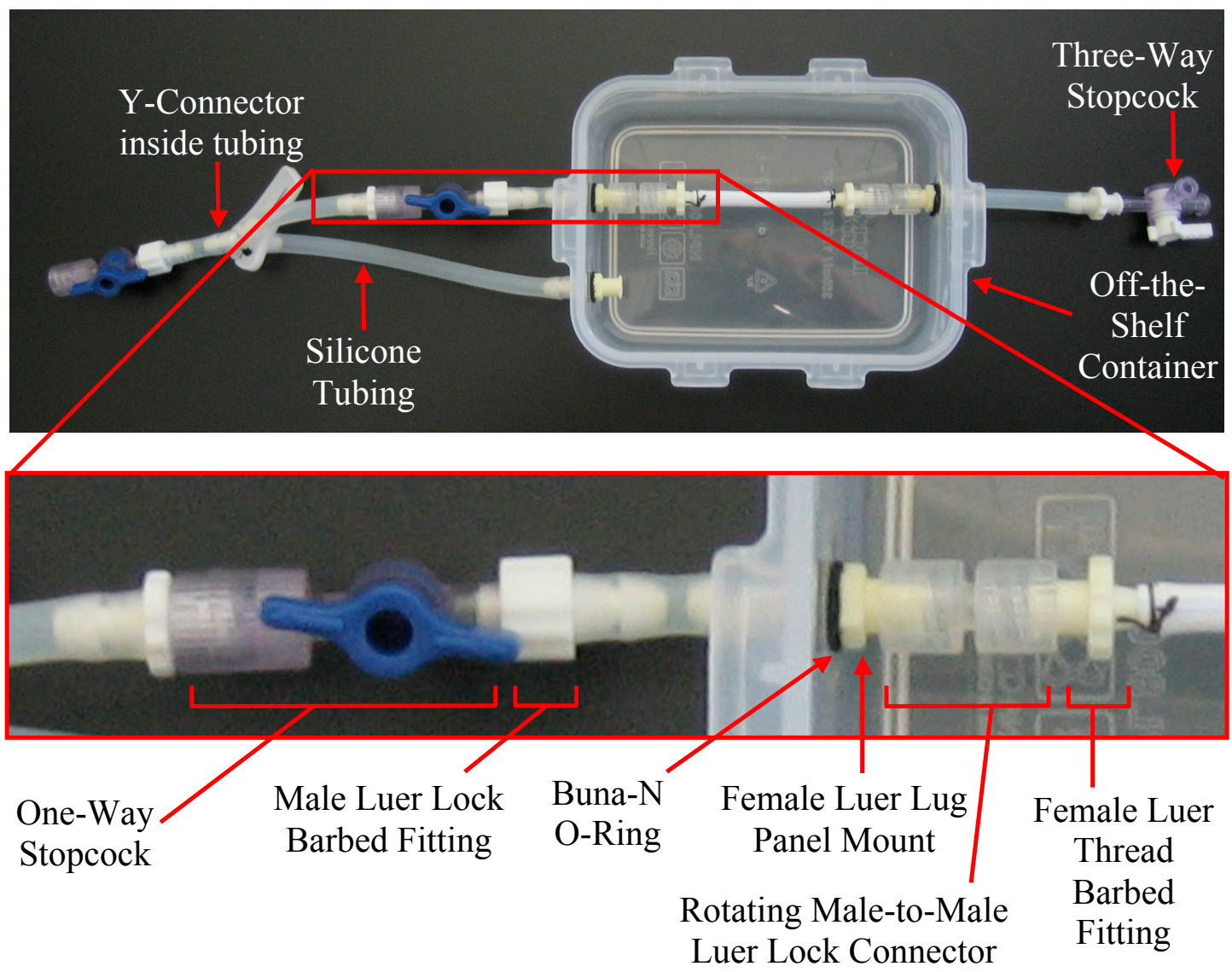

Figure 2.1. Current BVM bioreactor housing a straight scaffold. The bioreactor is made entirely of off-the-shelf components. Not pictured here is a snap-on sealable lid for the container. Not visible here are the O-rings placed on the external tip of the female luer lug panel mounts and lock nuts that secure the external O-rings in place. 


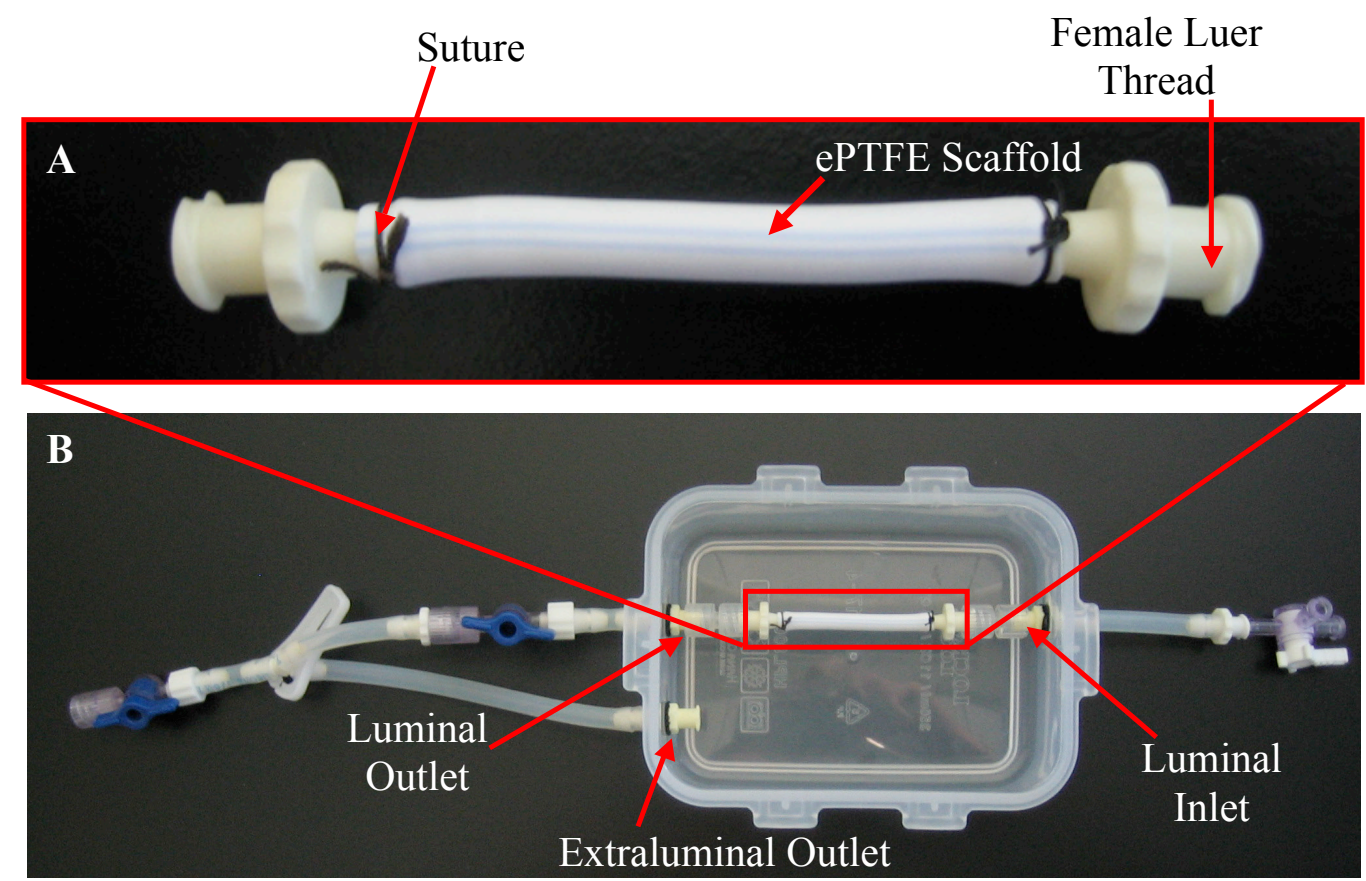

Figure 2.2. Current straight scaffold and pressure-sodding method. (A) The scaffold for BVMs is made of expanded polytetrafluoroethylene (ePTFE), a porous synthetic polymer. The scaffold has a blue double stripe across the top and is sutured to two fittings. The inner diameter (ID) of human coronary arteries may range between 1.9 and $4.5 \mathrm{~mm}$ [72], so the scaffold has an ID of $4 \mathrm{~mm}$. Cells are deposited onto the scaffold using a method known as pressure-sodding: cells are first suspended in media, and the cell suspension is then placed in a syringe; the luminal outlet of the bioreactor $(\mathrm{B}$, Luminal Outlet) is then closed, the syringe is attached to the luminal inlet ( $\mathrm{B}$, Luminal Inlet), the cell suspension is pushed into the scaffold lumen, the entire cell suspension except the cells is forced out the scaffold pores, and the cells remain lodged in the scaffold pores. 


\subsection{Background Information: Previous Designs}

Previous designs were proposed for complex-shaped scaffolds and bioreactors. Because the ePTFE scaffold currently used to develop straight BVMs is flexible, previous designs proposed that straight ePTFE scaffolds simply be bent into coronary geometries. To mimic the specific coronary bends defined by the American College of Cardiology and the American Heart Association, previous designs proposed three geometries for bent scaffolds: a scaffold bent less than 45 degrees, a scaffold bent 90 degrees, and a scaffold bent 180 degrees [71]. Some coronary bifurcations observed in clinical practice are T-shaped [73], and previous designs proposed that two straight ePTFE scaffolds be sutured together in a T-shape to mimic a T-shaped coronary bifurcation (Figure 2.3 A). Other coronary bifurcations observed in clinical practice are Y-shaped [73], and previous designs proposed that three straight ePTFE scaffolds be sutured together in a Y-shape to mimic a Y-shaped coronary bifurcation (Figure 2.3 B). Also, previous designs proposed that 5 bioreactors be developed, each tailored to house 1 of the 5 proposed scaffold geometries (Figure 2.4).

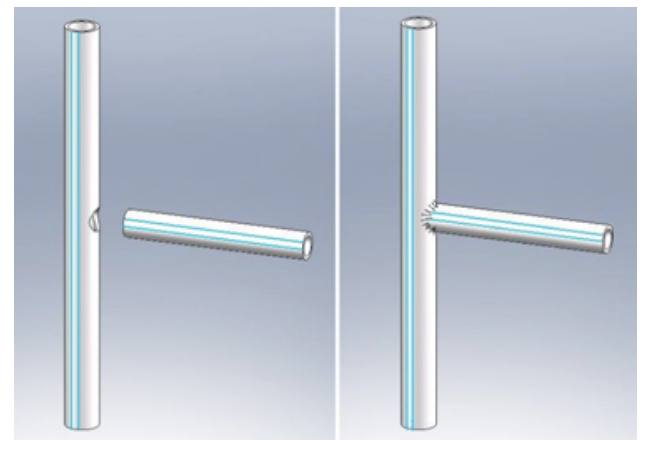

A

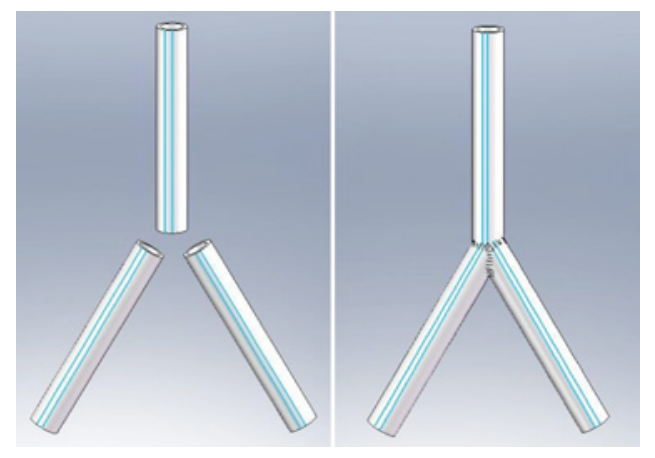

B

Figure 2.3. Construction methods for a T-shaped scaffold (A) and a Y-shaped scaffold (B) that were proposed in previous designs. [71] 


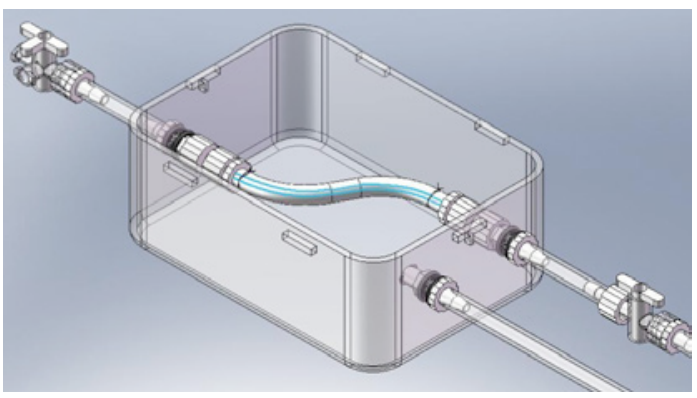

A

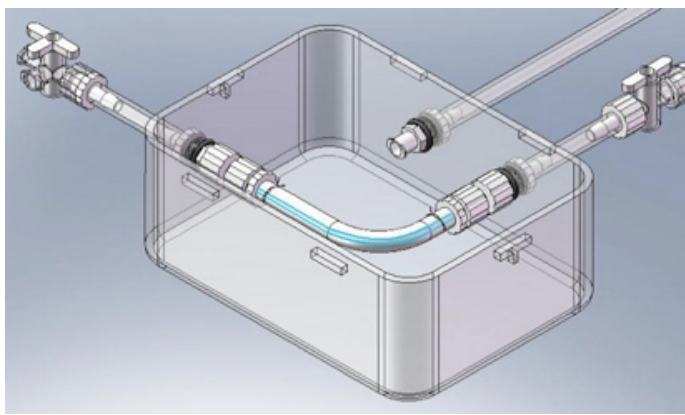

B

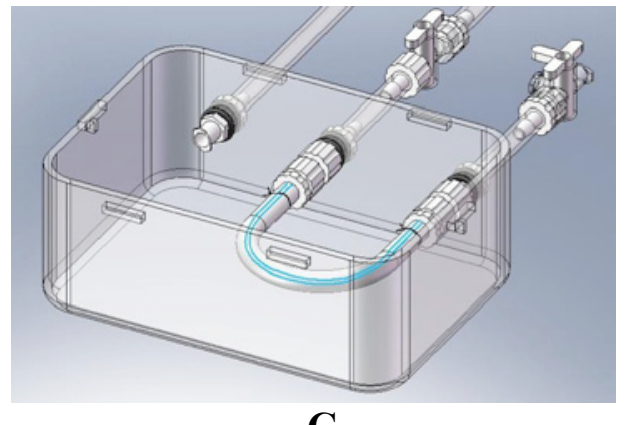

C

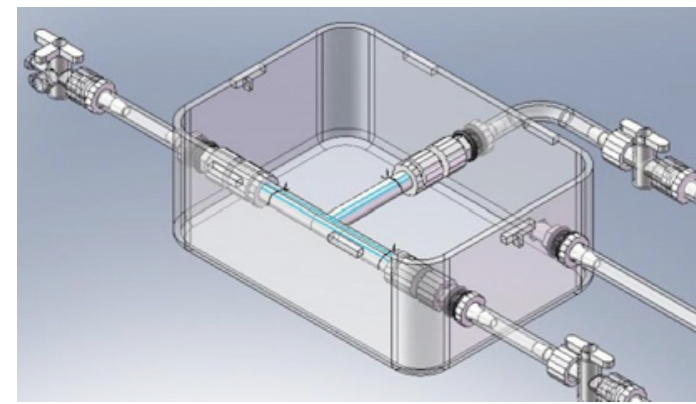

D

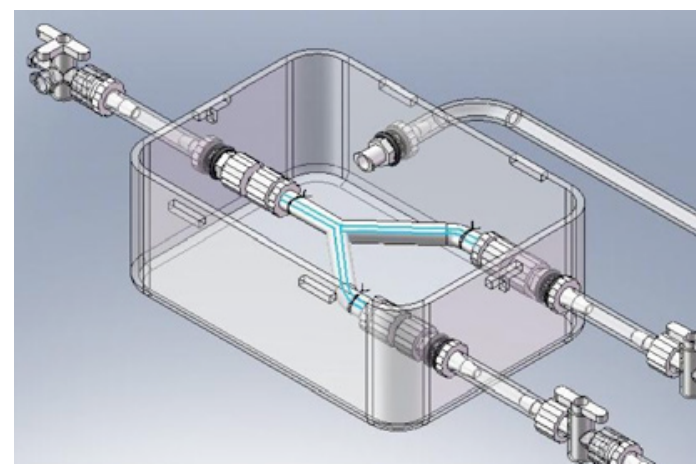

E

Figure 2.4. Previously proposed designs for bioreactors that can house complex-shaped scaffolds. Each bioreactor is tailored to house a specific scaffold geometry. A bioreactor was designed to house a scaffold bent less than 45 degrees, which will now be referred to as an S-shaped scaffold (A), another bioreactor was designed to house a scaffold bent 90 degrees, which will now be referred to as an L-shaped scaffold (B), another bioreactor was designed to house a scaffold bent 180 degrees, which will now be referred to as a Ushaped scaffold (C), another bioreactor was designed to house a T-shaped scaffold (D), and another bioreactor was designed to house a Y-shaped scaffold (E). [71] 


\subsection{Materials and Methods}

To create new scaffolds and bioreactors, new designs were first established. Then the scaffolds and bioreactors were constructed, human endothelial cells were deposited onto the complex-shaped scaffolds, the cell-lined scaffolds were cultivated inside the new bioreactors, and finally cell coverage of complex-shaped scaffolds was assessed to determine whether cells deposited on the complex-shaped scaffolds.

\subsubsection{Challenges with Previous Designs}

There are challenges that could arise during construction and use of the proposed Y-shaped scaffold. Construction of the proposed bifurcated scaffolds will involve suturing by hand, and the suturing proposed in Figure $2.3 \mathrm{~A}$ and B could be tedious, especially the suturing proposed for the Y-shaped scaffold, thereby increasing the difficulty of scaffold construction. Therefore, a simpler scaffold construction method should be developed at least for the Y-shaped scaffold. Also, the suture sites in bifurcated scaffolds may be susceptible to unwanted leakage when fluid moves through the scaffolds, and the Y-shaped scaffold may be more susceptible to leakage than the Tshaped scaffold because the Y-shaped scaffold has more sutures. Therefore, the number of sutures at least in the Y-shaped scaffold should be reduced.

There are also challenges that could arise during construction and use of the proposed bioreactors. Researchers may want to run multiple stent-testing trials in each of the five proposed geometries. For example, trials in T-shaped BVMs could occur one week, trials in U-shaped BVMs could occur another week, and trials in L-shaped BVMs could occur another week. Researchers would have to construct, store, and maintain a 
separate set of bioreactors for each scaffold geometry, which could result in significant bioreactor construction time, need for significant storage space for the many bioreactors, and significant bioreactor maintenance time. Instead, if researchers had bioreactors that were each capable of housing all five proposed scaffold geometries, researchers could construct a smaller number of these multifunctional bioreactors and reuse the same multifunctional bioreactors each week, which would reduce the number of bioreactors that must be constructed, stored, and maintained, thereby reducing bioreactor construction time, the need for storage space, and bioreactor maintenance time. Therefore, a multifunctional bioreactor was developed.

Because of the challenges that could arise during construction and use of particularly the Y-shaped scaffold proposed in previous designs and during construction and use of the five bioreactors proposed in previous designs, a new design was created for the Y-shaped scaffold, and a design was created for a multifunctional bioreactor. Dimensions and materials were then specified for all designs, resulting in final designs for complex-shaped scaffolds in all five geometries and final designs for bioreactors that could house all five scaffold geometries.

\subsubsection{Final Designs for Complex-Shaped Scaffolds}

The new design for the Y-shaped scaffold is described as follows: two straight ePTFE scaffolds were sutured together to form a T-shaped scaffold using the same method as depicted in Figure 2.3 A, then each end of the crossbar of the " $\mathrm{T}$ " was bent upwards so that the "T" became a "Y", resulting in a Y-shaped scaffold with fewer sutures than the Y-shaped scaffold proposed in previous designs. With a reduced number 
of sutures, this new design for a Y-shaped scaffold may make Y-shaped scaffold construction less tedious and may reduce the chance of unwanted leakage. Because this new design reduced the possible challenges that could occur with the previous design for a Y-shaped scaffold, this new design became the final design for a Y-shaped scaffold. The previous designs for the other four complex-shaped scaffolds became the final designs for those scaffolds.

Dimensions of the complex-shaped scaffolds are listed as follows. The total lengths of S-, L-, and U-shaped scaffolds were $7 \mathrm{~cm}, 5 \mathrm{~cm}$, and $5.5 \mathrm{~cm}$ respectively. The stem of the " $\mathrm{T}$ " on a $\mathrm{T}$-shaped scaffold was $2.5 \mathrm{~cm}$ long, and the crossbar of the " $\mathrm{T}$ " was $6 \mathrm{~cm}$ long. Another T-shaped scaffold was bent into a Y-shape without changing any dimensions to create a Y-shaped scaffold. All scaffolds had 4-mm IDs.

\subsubsection{Final Designs for Bioreactors That Can House Complex-Shaped Scaffolds}

A multifunctional bioreactor was designed (Figure 2.5). Because this multifunctional bioreactor design eliminates the possible challenges that could occur with the previous bioreactor designs, this design became a final bioreactor design. The multifunctional bioreactor was designed without the ability to house Y-shaped scaffolds because additional inlets, outlets, or tubing would crowd the bioreactor, making it difficult to use; therefore, the previous design for a bioreactor capable of housing only a Y-shaped scaffold also became a final bioreactor design (Figure 2.6). To be able to develop BVMs in all five proposed geometries, two bioreactors were constructed: a multifunctional bioreactor and a bioreactor capable of housing only a Y-shaped scaffold. 

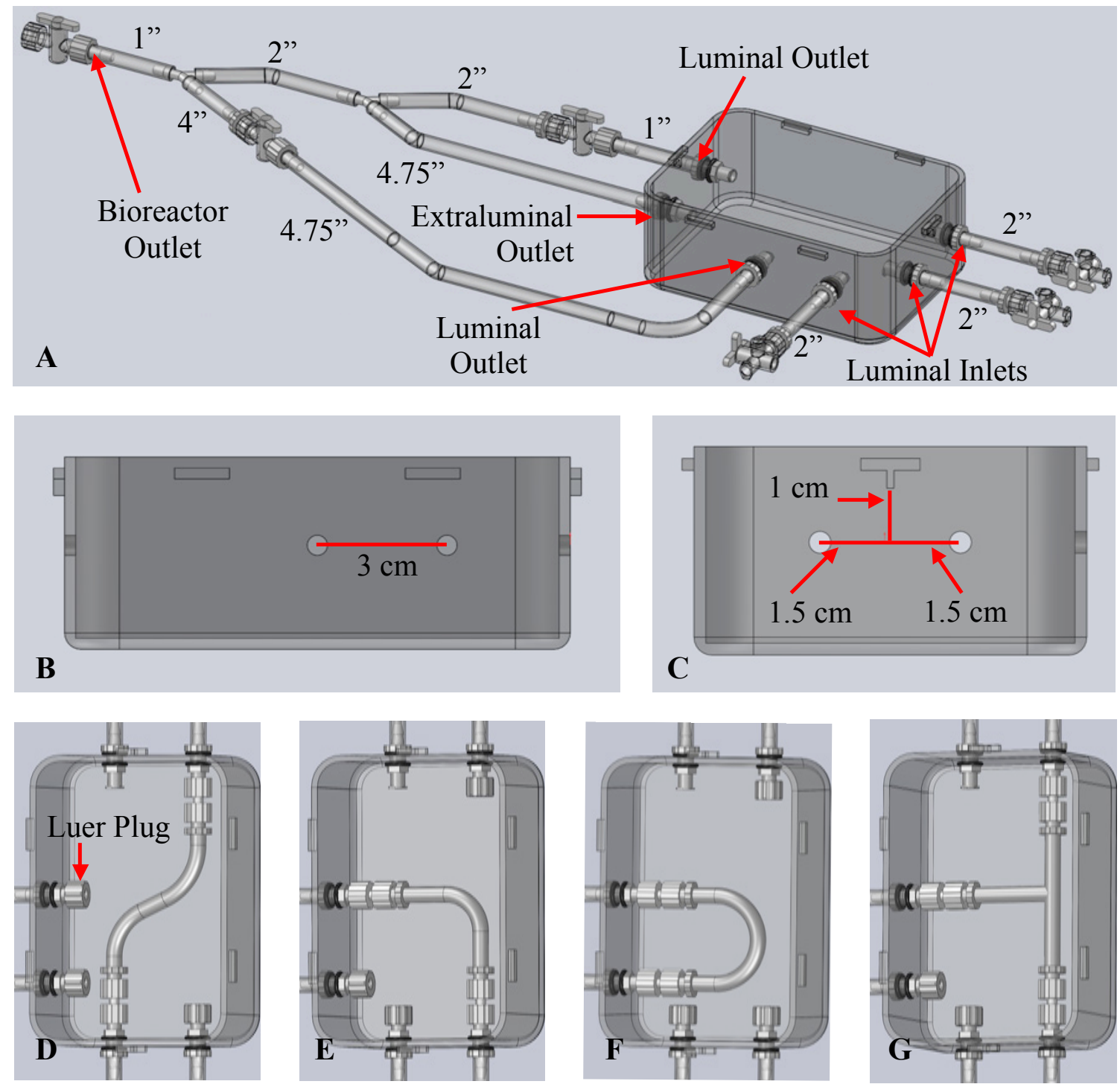

Figure 2.5. Multifunctional Bioreactor. New positions for inlets and outlets and new tubing arrangements and dimensions were designed $(\mathrm{A}-\mathrm{C})$; all inlets and outlets are the same distance from the top of the container, the luminal outlet is horizontally centered on its wall, and $\mathrm{C}$ represents both short sides of the container. The multifunctional bioreactor houses one scaffold at a time, and any inlets or outlets not being used when a scaffold is inserted will be capped off with luer plugs (D-G). Since the FDA has recommended that vessels simulating a worst-case bend for coronary stent testing have a $15-\mathrm{mm}$ radius of curvature, any inserted U-shaped scaffold will have a 15-mm radius of curvature. 

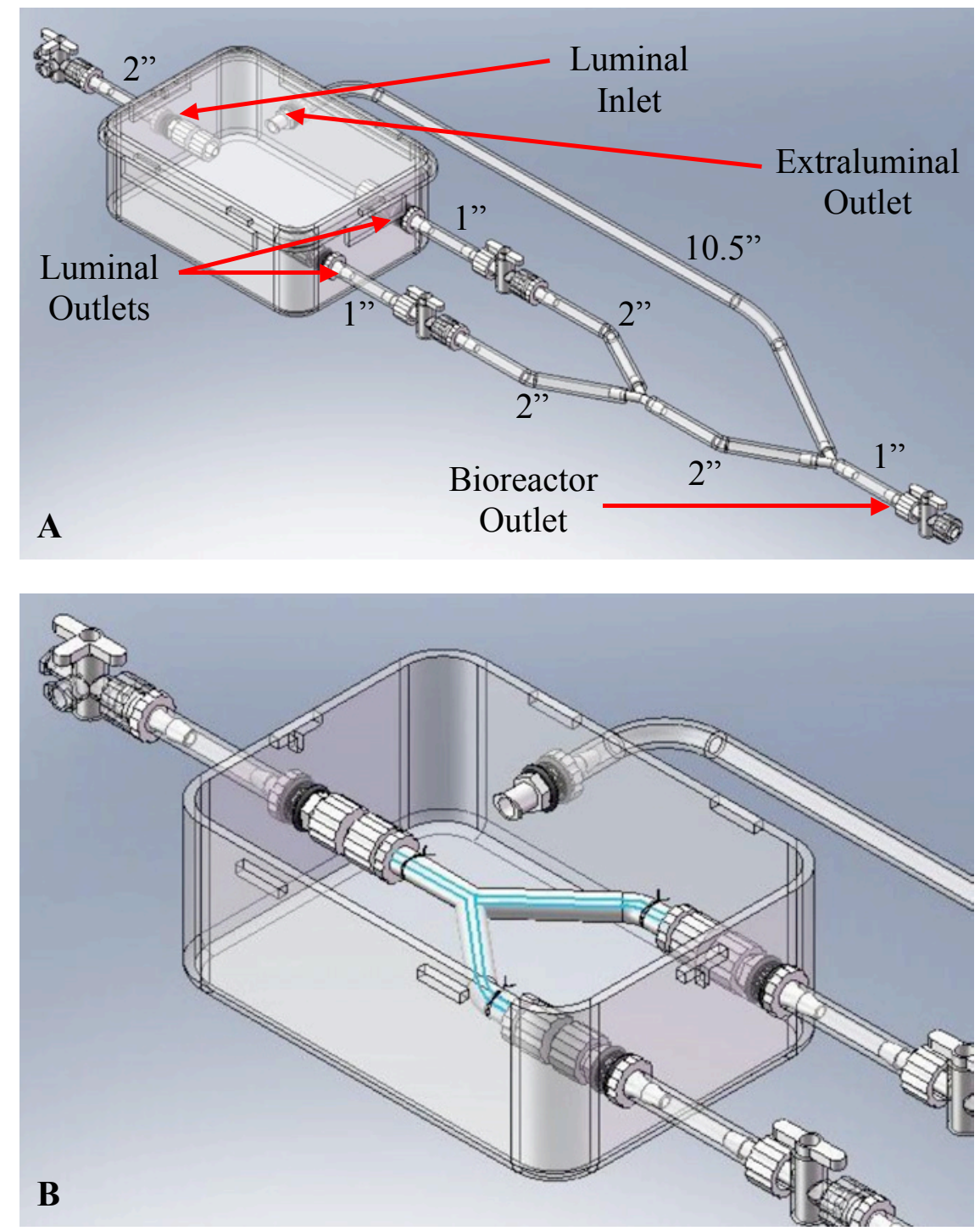

Figure 2.6. Bioreactor capable of housing only a Y-shaped scaffold. The general arrangement of inlets, outlets, and tubing for this bioreactor were established in previous designs, but specific dimensions were not established. Therefore, the design was updated to include tubing dimensions (A) and specific locations for all inlets and outlets: the luminal outlets will be $3 \mathrm{~cm}$ apart, the luminal inlet is horizontally centered on its wall, the extraluminal outlet is $3 \mathrm{~cm}$ horizontal from the centerline on its wall, and all inlets and outlets are located the same distance away from the top of the container as depicted in Figure 2.5 C. (B). 


\subsubsection{Construction of Complex-Shaped Scaffolds}

All scaffolds were made of tubular ePTFE with 4-mm IDs (Impra Bard, Inc., Tempe, AZ). Latex gloves were worn when handling the ePTFE. All bent scaffolds were constructed by simply bending straight pieces of ePTFE into complex shapes: a straight ePTFE scaffold that was $7 \mathrm{~cm}$ in length was bent into an S-shape, creating an S-shaped scaffold; a straight ePTFE scaffold that was $5 \mathrm{~cm}$ in length was bent into an L-shape, creating an L-shaped scaffold; and a straight ePTFE scaffold that was $5.5 \mathrm{~cm}$ in length was bent into a U-shape, creating a U-shaped scaffold. A T-shaped scaffold was constructed as follows: first, a hole that was $4 \mathrm{~mm}$ in diameter was cut out of the center of a straight ePTFE scaffold that was $6 \mathrm{~cm}$ in length, then the end of another straight ePTFE scaffold that was $2.5 \mathrm{~cm}$ in length was sutured (6.0 silk suture) onto the hole, creating a T-shaped scaffold (Figure 2.7). Another T-shaped scaffold was constructed identically to the first one, and each end of the crossbar of the "T" was bent upward to form a "Y", creating a Y-shaped scaffold. The ends of all scaffolds were sutured (2.0 silk suture) onto female luer thread barbed fittings (white nylon, 200 series barb, 1/8" ID, Value Plastics, Part Number FTLL230-1) as shown in figure 2.2 A. 

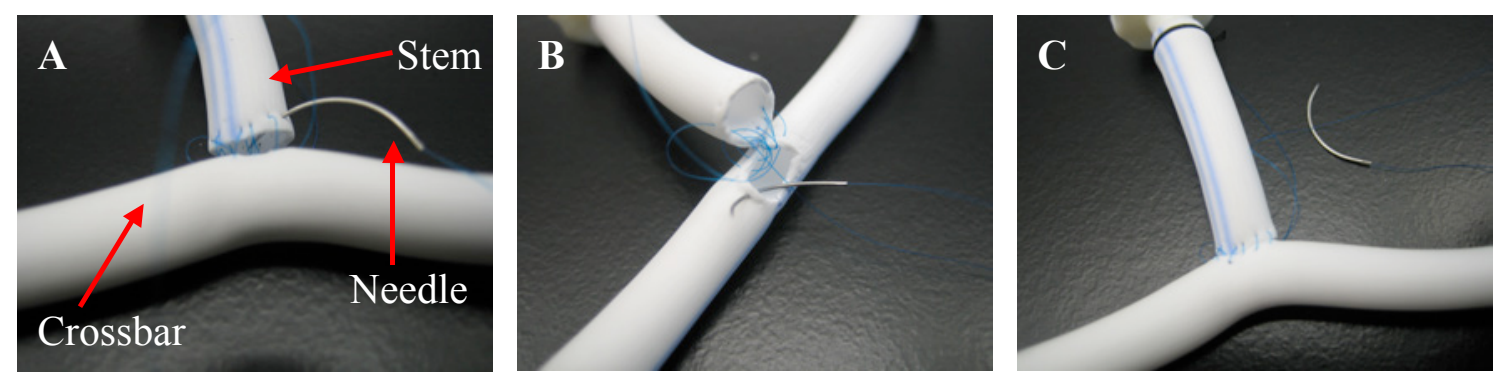

Figure 2.7. Construction of T-shaped scaffold. To suture together two straight pieces of ePTFE, the following procedure was followed. First, a suture needle was used to puncture the stem (A). The needle was then pushed through the puncture and pulled out the stem end that was being sutured. The needle tip was then used to puncture the crossbar as shown in B, and the needle tip was pulled through the puncture. The needle tip was then used to puncture the stem again in a location that was $1 \mathrm{~mm}$ next to the previous puncture in the stem, and this technique was repeated around the entire suture site $(C)$. The suture was then pulled taut, and 6 overhand knots were tied to secure the suture.

\subsubsection{Construction of Bioreactors to House Complex-Shaped Scaffolds}

The bioreactor that currently houses only straight BVMs (Figure 2.1) is made of an off-the-shelf polypropylene container, a number of small off-the-shelf plastic parts known as fittings, and silicone tubing. Detailed assembly instructions for that bioreactor are located in Design and Optimization of a Blood Vessel Mimic Bioreactor System for the Evaluation of Intravascular Devices in Simple and Complex Geometries on pages 8493 and 212-218 [71]. Those assembly instructions detail the methods for drilling holes in the container, attaching fittings, and attaching tubing to the fittings. Identical assembly methods were used for construction of the multifunctional bioreactor and for construction of the bioreactor capable of housing only Y-shaped scaffolds. But in the case of the 
multifunctional bioreactor and the bioreactor capable of housing only Y-shaped scaffolds, holes were drilled for inlets and outlets in the locations described in Figures 2.5 A and 2.6 A, fittings were attached in the locations depicted in those figures, and tubing was cut with the dimensions depicted in those figures and was placed in the locations depicted in those figures. All holes were drilled with a handheld power drill and a 15/64" drill bit. The exact materials used to construct each bioreactor are listed in Tables 2.1 and 2.2. A protocol for maintenance of multifunctional bioreactors is located in Appendix B. 
Table 2.1. Materials for Multifunctional Bioreactor

\begin{tabular}{|c|c|c|c|}
\hline Component & Company & Part Number & Quantity \\
\hline Polypropylene Container & Lock \& Lock & HPL806 & 1 \\
\hline 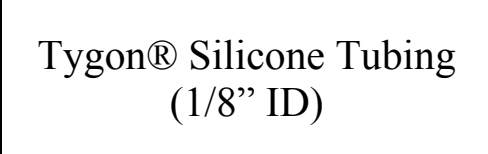 & Cole-Parmer & WU-95702-06 & $\begin{array}{l}25.5 \text { inches cut } \\
\text { according to } \\
\text { Figure } 2.5 \mathrm{~A}\end{array}$ \\
\hline Discofix ${ }^{\circledR}$ 1-Way Stopcock & B. Braun Medical & D100 455980 & 3 \\
\hline 3-Way Stopcock & Value Plastics & VPB1000062N & 3 \\
\hline $\begin{array}{l}\text { Female Luer Lug Panel } \\
\text { Mount (1/8" Barb) }\end{array}$ & Value Plastics & FTLB230-1 & 6 \\
\hline Buna N O-Ring & McMaster-Carr & $2418 \mathrm{~T} 112$ & 12 \\
\hline Lock Nut & Value Plastics & LNS-1 & 6 \\
\hline Luer Plug & Value Plastics & MTLLP-1 & 3 \\
\hline $\begin{array}{l}\text { Female Luer Thread Fitting } \\
\text { (1/8" Barb) }\end{array}$ & Value Plastics & FTLL230-1 & 5 \\
\hline $\begin{array}{l}\text { Male Luer Lock Fitting } \\
\text { (1/8" Barb) }\end{array}$ & Value Plastics & MTLL230-1 & 3 \\
\hline Y-Connector (1/8” Barb) & Value Plastics & Y230-1 & 2 \\
\hline $\begin{array}{l}\text { Rotating Luer Lock } \\
\text { Connector }\end{array}$ & Qosina & 17644 & $\begin{array}{c}2 \text { or } 3 \text { for bent } \\
\text { or T-shaped } \\
\text { scaffold } \\
\text { respectively }\end{array}$ \\
\hline
\end{tabular}


Table 2.2. Materials for Bioreactor Capable of Housing Only Y-shaped Scaffolds

\begin{tabular}{|c|c|c|c|}
\hline Component & Company & Part Number & Quantity \\
\hline Polypropylene Container & Lock \& Lock & HPL806 & 1 \\
\hline $\begin{array}{c}\text { Tygon }{ }^{\circledR} \text { Silicone Tubing } \\
(1 / 8 " \mathrm{ID})\end{array}$ & Cole-Parmer & WU-95702-06 & $\begin{array}{l}21.5 \text { inches cut } \\
\text { according to } \\
\text { Figure } 2.6 \mathrm{~A}\end{array}$ \\
\hline Discofix ${ }^{\circledR}$ 1-Way Stopcock & B. Braun Medical & D100 455980 & 3 \\
\hline 3-Way Stopcock & Value Plastics & VPB1000062N & 1 \\
\hline $\begin{array}{l}\text { Female Luer Lug Panel } \\
\text { Mount (1/8" Barb) }\end{array}$ & Value Plastics & FTLB230-1 & 4 \\
\hline Buna N O-Ring & McMaster-Carr & 2418T112 & 8 \\
\hline Lock Nut & Value Plastics & LNS-1 & 4 \\
\hline $\begin{array}{l}\text { Female Luer Thread Fitting } \\
\qquad(1 / 8 \text { " Barb })\end{array}$ & Value Plastics & FTLL230-1 & 3 \\
\hline $\begin{array}{l}\text { Male Luer Lock Fitting } \\
\text { (1/8" Barb) }\end{array}$ & Value Plastics & MTLL230-1 & 3 \\
\hline Y-Connector (1/8" Barb) & Value Plastics & Y230-1 & 2 \\
\hline $\begin{array}{l}\text { Rotating Luer Lock } \\
\text { Connector }\end{array}$ & Qosina & 17644 & 3 \\
\hline
\end{tabular}

\subsubsection{Validation of New Scaffolds and Bioreactors}

After construction, the new scaffolds and bioreactors were tested for the ability to generate complex-shaped BVMs. If cell deposition occurred on the new scaffolds inside the new bioreactors, the designs for the new scaffolds and bioreactors would be validated.

Only bent scaffolds and multifunctional bioreactors were tested for the ability to generate complex-shaped BVMs, and the reason for this is as follows. To be useful for coronary stent testing, complex-shaped BVMs need other characteristics in addition to 
cells deposited on their scaffolds, such as a confluent cell lining and a cell lining that is not completely denuded after coronary stent deployment. Development of these other characteristics will be discussed in future chapters. But the laboratory techniques for developing these other characteristics are likely similar, regardless of the geometry. Therefore, instead of testing techniques in all five scaffold geometries, only some geometries were first tested to discover techniques that could be used with all scaffold geometries. This saved significant time and resources and allowed faster discovery of successful techniques. Bent scaffolds were chosen as the scaffolds to first test because the suture sites of bifurcated scaffolds presented potential challenges, and these potential challenges could prolong discovery of successful techniques that could be used with all scaffold geometries.

To test bent scaffolds and multifunctional bioreactors for the ability to generate complex-shaped BVMs, the following steps were carried out. (Note: the following steps only summarize the many tissue engineering laboratory steps that must be carried out when generating BVMs and that were carried out for this validation test; the many tissue engineering laboratory steps will be discussed in future chapters.) First, an S-, L-, and Ushaped scaffold were each inserted into a multifunctional bioreactor. The scaffolds and bioreactors were primed with media, and HUVECs were pressure-sodded onto each scaffold. The bioreactors were attached to media reservoirs, placed in an incubator at $37^{\circ} \mathrm{C}$ and $5 \% \mathrm{CO}_{2}$, and connected to an 8-roller pump that circulated media through the BVMs for 1 day. Then each vessel was removed from its bioreactor, fixed in Histochoice, cut in half longitudinally, and stained with the fluorescent nuclear stain bisbenzimide (BBI). A fluorescent microscope was used to capture en face images of the luminal 
surface of each scaffold, and the images were used to determine whether cells deposited on each scaffold.

\subsection{Results}

Construction of all five complex-shaped scaffolds and the two new bioreactors yielded prototypes that will be discussed here. The BBI images that resulted from the validation testing of all three bent scaffolds will also be discussed here.

\subsubsection{Prototypes of New Scaffolds and Bioreactors}

Construction of bent scaffolds involved nothing more than bending straight scaffolds into complex geometries, which was easy. Construction of bifurcated scaffolds was also easy: cutting a hole in the middle of a straight scaffold with a razor, puncturing ePTFE with a suture needle, placing ePTFE punctures $1 \mathrm{~mm}$ next to each other, and tying 6 overhand knots to secure the sutures were all easy steps. Suturing together two straight pieces of ePTFE was a successful method for attaching the two pieces and for creating a T-shaped scaffold. Bending both ends of the crossbar of a T-shaped scaffold upward was an easy and successful method for creating a Y-shaped scaffold. Placing female luer thread barbed fittings inside the ends of all scaffolds and suturing the ends of all scaffolds to the female luer thread barbed fittings was also easy.

Construction of the multifunctional bioreactor and the bioreactor capable of housing only Y-shaped scaffolds was similarly easy. Construction of these new bioreactors varied only slightly from construction of the current straight-BVM bioreactor: for the new bioreactors, additional holes were drilled into the polypropylene container, 
tubing was cut in different dimensions and attached at different locations, a few more stopcocks were added, and luer plugs were used to cap off any inlets or outlets not being used when complex-shaped scaffolds were inserted. All stopcocks on both bioreactors were easily accessible, therefore the additional tubing on both bioreactors did not crowd the bioreactors to the point of stopcocks being difficult to access.

Insertion of all five complex-shaped scaffolds into the new bioreactors was a success. The female luer thread barbed fittings on the ends of each complex-shaped scaffold were easy to secure into position on the male-to-male rotating fittings. In the multifunctional bioreactor, luer plugs were easy to attach to inlets or outlets not being used, and no media flowed through any of the capped-off inlets or outlets in any of the three bioreactors throughout the entire validation test. No scaffolds experienced any kinking. Prototypes of the new scaffolds and bioreactors are shown in Figure 2.9. 

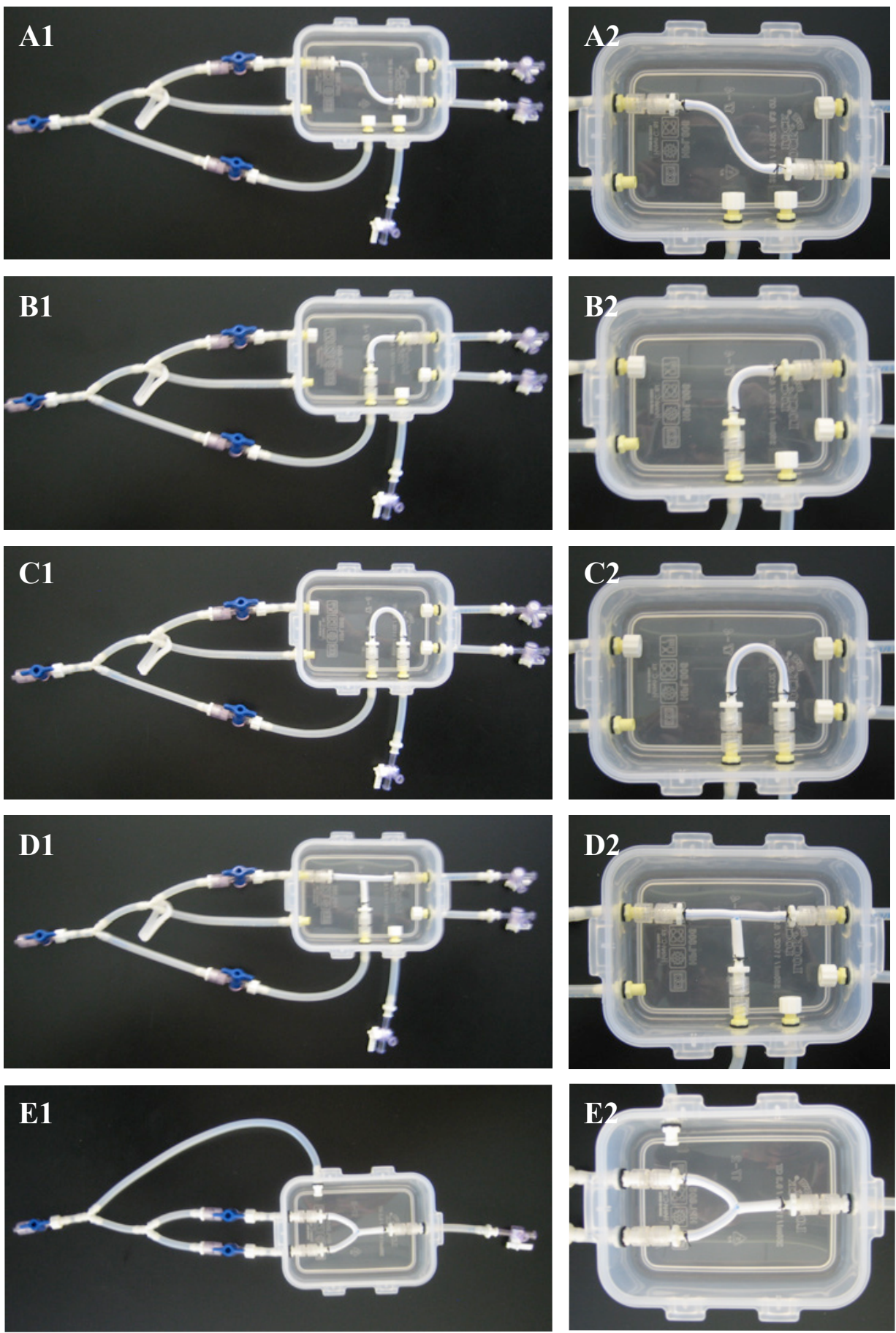

Figure 2.9. Prototypes of the new scaffolds and bioreactors. The multifunctional bioreactor houses an S-shaped scaffold (A1-A2), an L-shaped scaffold (B1-B2), a Ushaped scaffold (C1-C2), and a T-shaped scaffold (D1-D2). The bioreactor in E1-E2 houses only a Y-shaped scaffold. Bioreactor lids were removed for scaffold visibility. 


\subsubsection{Cell Deposition on Complex-Shaped Scaffolds}

The bent scaffolds and multifunctional bioreactor generated S-, L-, and U-shaped BVMs, and all BVMs exhibited cell deposition in BBI images (Figure 2.10).

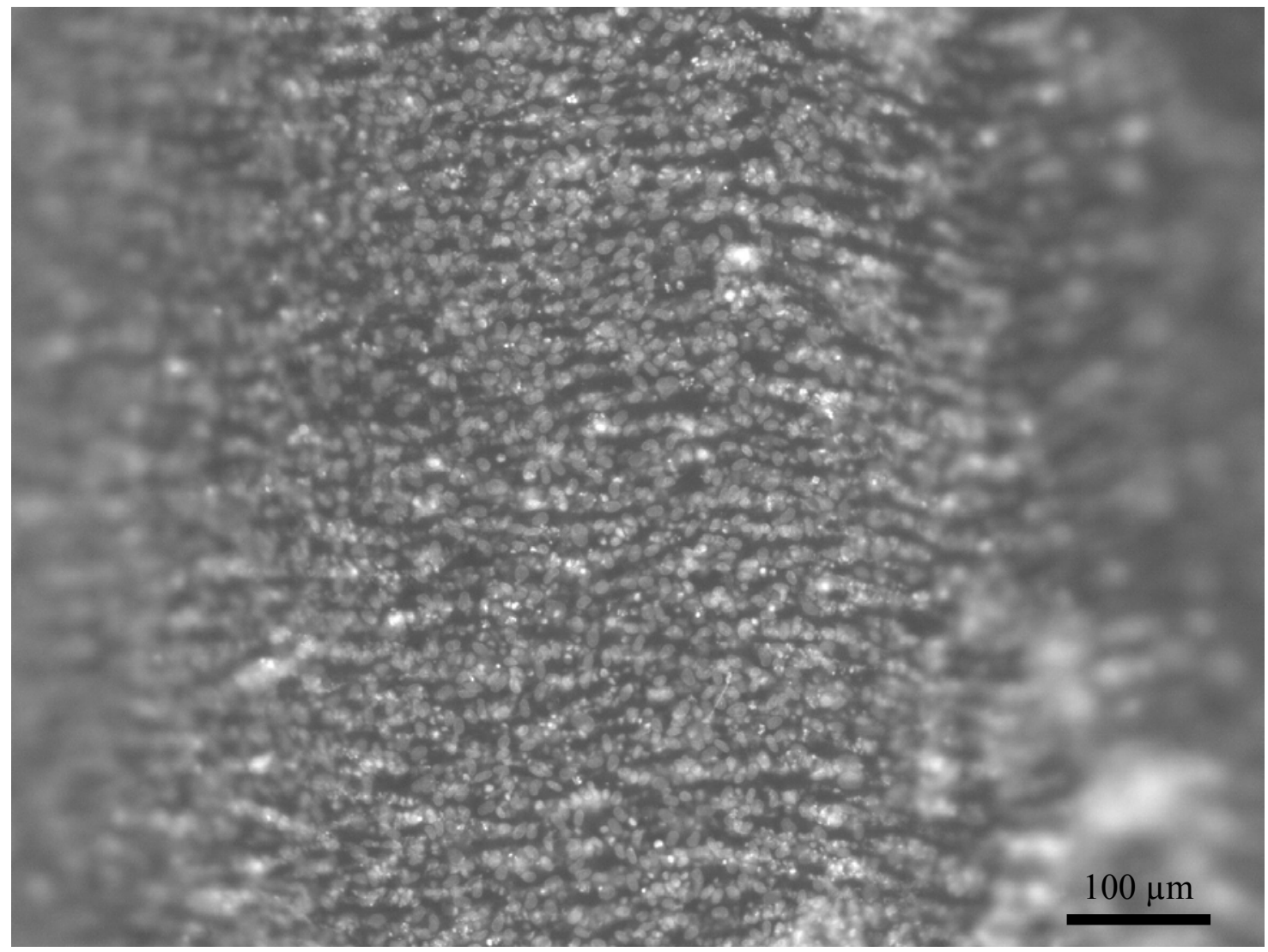

Figure 2.10. BBI image showing cell deposition on U-shaped scaffold. BBI causes cell nuclei to fluoresce, and en face BBI images show many small dots that each correspond to the nucleus of a cell. The many small dots are usually colored blue, but because this is a black-and-white image, the many small dots are gray. Five en face BBI images were taken of each complex-shaped scaffold, and all 5 images for each scaffold showed cell deposition similar to that shown in this image. 


\subsection{Discussion and Conclusions}

Multiple initial steps were taken toward the development of BVMs in complex geometries. Previous designs for complex-shaped scaffolds and bioreactors were modified and given specific dimensions. The Y-shaped scaffold was redesigned to make construction less tedious and to reduce unwanted leakage when fluid moves through the Y-shaped scaffold. A multifunctional bioreactor was designed to reduce bioreactor construction time, the need for bioreactor storage space, and bioreactor maintenance time. Construction of the new scaffolds and bioreactors, insertion of the new scaffolds into the new bioreactors, and capping off unused inlets or outlets in multifunctional bioreactors were all easily performed. Cells deposited on S-, L-, and U-shaped scaffolds inside multifunctional bioreactors, validating the design of these scaffolds and the multifunctional bioreactor. These findings support the hypothesis that complex-shaped scaffolds could be designed and constructed, that bioreactors capable of housing the new scaffolds could be designed and constructed, and that cells would deposit onto the new scaffolds inside the new bioreactors.

Although BBI images showed cell deposition on each bent scaffold, nothing else about the cell linings was assessed. It is possible that the cell lining on each bent scaffold has characteristics that do not make it useful for coronary stent testing. For example, cells may have densely deposited in some regions of each bent scaffold but sparsely deposited in other regions. Regions of sparse cell deposition may lead to formation of subconfluent cell linings in those regions, which could lead to inaccurate assessments of reendothelialization of coronary stents. Therefore, the cell lining on each bent scaffold 
should be evaluated more closely to determine how well it actually mimics the endothelium of native blood vessels.

Before the cell lining on each bent scaffold is evaluated more closely, an alternative step is to validate the bifurcated scaffolds. But at this point, validation of the bifurcated scaffolds may simply prolong the development of any fully functional complex-shaped BVM. Closer evaluation of the cell lining on each bent scaffold may provide information that is vital to the development of successful techniques that could be used with all scaffold geometries, thereby expediting development of a fully functional complex-shaped BVM. Therefore, bifurcated scaffolds were not validated at this point.

In conclusion, complex-shaped scaffolds can be constructed, bioreactors capable of housing complex-shaped scaffolds can be constructed, and endothelial cells can be deposited within complex-shaped scaffolds. The validated bent scaffolds and validated multifunctional bioreactors were next used to further develop complex-shaped BVMs. 


\section{Consistency of Cell Deposition on Complex-Shaped Scaffolds}

\subsection{Introduction}

This chapter addresses the second specific aim of this thesis: deposit cells throughout the entire inner surface of complex-shaped scaffolds.

There are multiple characteristics of straight BVMs that make them useful for stent testing. One of these characteristics is a confluent cell lining [31]. A native human blood vessel features an endothelium, which is a confluent lining of endothelial cells on the inner surface of the blood vessel. When a coronary stent is deployed in a coronary artery, the stent denudes the endothelium in the stented region $[51,68]$. Endothelial cells in the intact region of endothelium next to the stented region begin to migrate into the denuded region and eventually onto the stent [74], and this migration of endothelial cells is the predominant source of cells involved in re-endothelialization [74]. When a stent is deployed in a straight BVM, cells that have not been denuded from the cell lining of the BVM re-endothelialize the stent [31]. Therefore, complex-shaped BVMs should also be developed with a confluent endothelial cell lining so that a stent can denude some endothelial cells but still leave behind intact endothelial cells that will re-endothelialize the stent.

A confluent cell lining may be difficult to form on a complex-shaped scaffold, and the reason for this is described as follows. A confluent cell lining in a straight BVM does not form as soon as cells are pressure-sodded onto the ePTFE scaffold [59]. When endothelial cells are first deposited onto the scaffold, the cells are balled-up, and the cell lining is not confluent (Figure 3.1 A) [59]. After a few days of cultivation under flow, the 
balled-up cells begin to spread out [59], the cells begin to physically contact each other, and a confluent cell lining forms when no visible gaps exist between any cells (Figure 3.1 B). Although cells have deposited on each bent scaffold as described in the previous chapter, the consistency of that cell deposition throughout the inner surface of each scaffold is unknown - it is possible that cells deposited unevenly. Cells in regions with possibly dense cell deposition may have to only spread out to contact neighboring cells, whereas cells in regions with sparse cell deposition may have to spread out but also migrate to contact neighboring cells. Therefore, regions of dense cell deposition may form a confluent cell lining faster than regions of sparse cell deposition, possibly resulting in a complex-shaped BVM with a cell lining that is confluent in some regions and subconfluent in other regions. If a stent were deployed in one of these unevenly confluent complex-shaped BVMs, subconfluent regions of the BVM may not fully reendothelialize the stent, while confluent regions may fully re-endothelialize the stent, resulting in uneven re-endothelialization and possibly an inaccurate portrayal of that stent's ability to re-endothelialize. Therefore, it is desirable for cells to deposit evenly on a complex-shaped scaffold. 


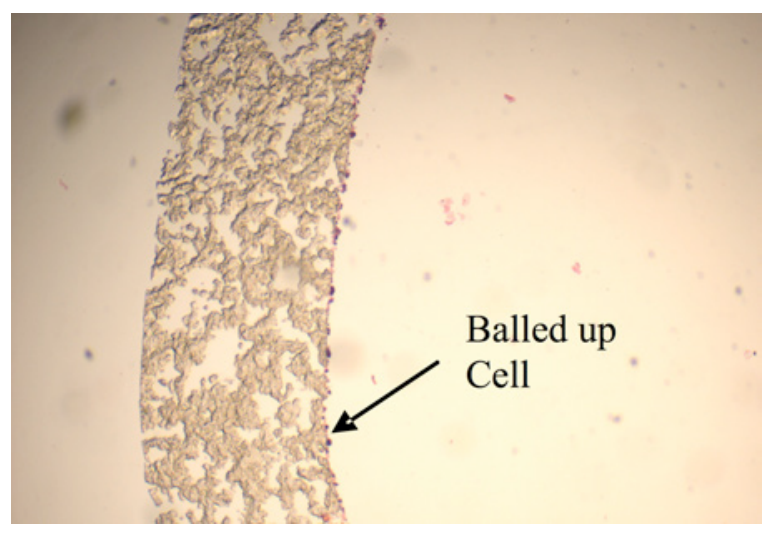

A

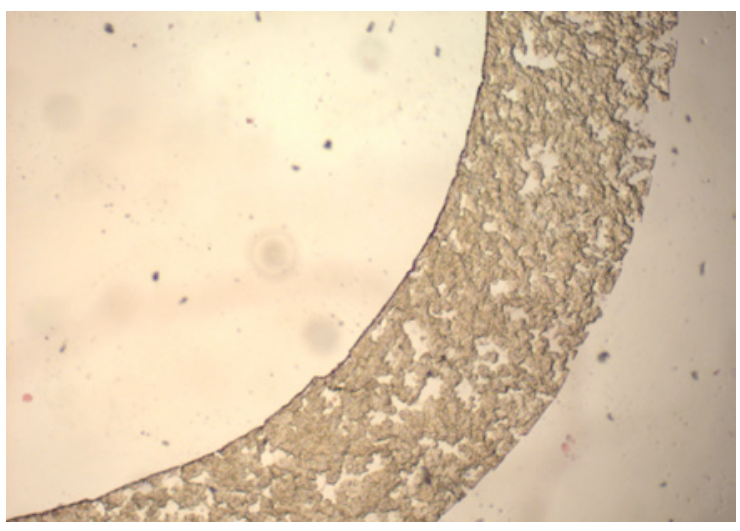

B

Figure 3.1. Cross-sections of straight BVM. The ePTFE scaffold is the large beige curve in each image, and the cells are small dark dots in A and a very thin dark line on the inner surface of the scaffold in B. (A) After 1 day of cultivation, cells appear balled-up, cell coverage on the scaffold is sparse, and the cell lining is not confluent. (B) After 7 days of cultivation, cells appear spread out, and the cell lining is confluent. [59]

There are various ways that cells may deposit unevenly on a complex-shaped scaffold. HUVECs will be pressure-sodded onto complex-shaped scaffolds, and when the cell suspension is injected into the complex geometries, cells may not deposit evenly along the length of the scaffolds. In a bent scaffold, cells may deposit only at the proximal portion of the bend, resulting in no cell lining at the distal end of the bend (Figure 3.2). In a bifurcated scaffold, the cell suspension could completely leak out of the suture sites and never reach the vessel segments after the bifurcation, resulting in no cell lining after the bifurcation. On L- and U-shaped scaffolds, cells may deposit more densely on the outer wall of the bends than on the inner wall of the bends. When all complex-shaped scaffolds are inserted horizontally into their bioreactors, cells may deposit more densely on the bottom half of the scaffolds than on the top half of the 
scaffolds due to the force of gravity pulling cells downward during pressure-sodding. For these reasons, cell deposition on complex-shaped scaffolds was assessed in more detail in this chapter.
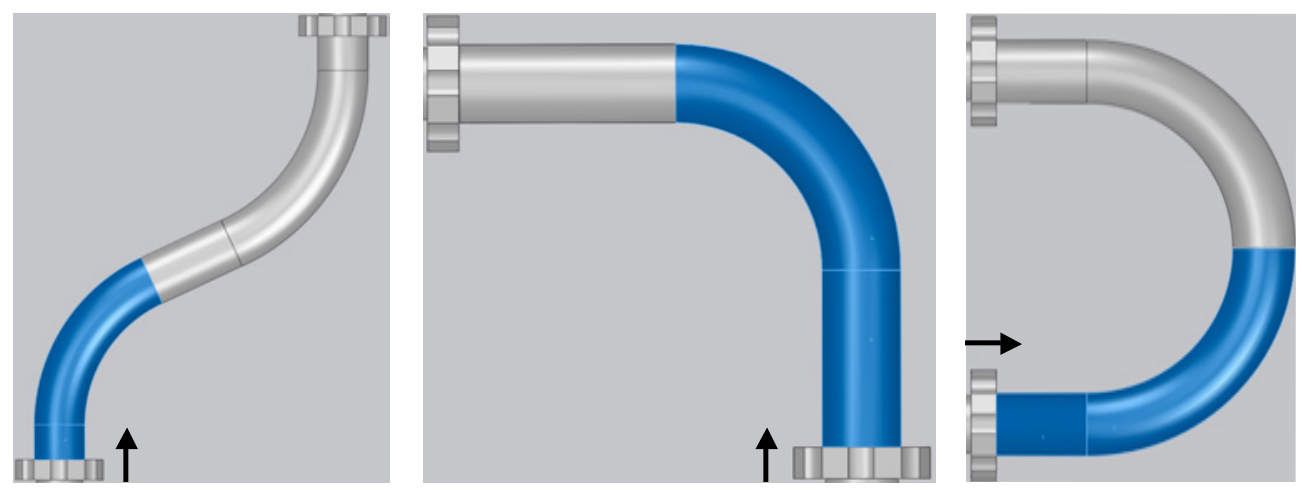

Figure 3.2. Possible cell deposition in bent scaffolds. Black arrows indicate the flow direction of the injected cell suspension. Cells may deposit only in the proximal blue regions.

To save time and resources and allow for faster discovery of successful laboratory techniques as in the previous chapter, only L- and U-shaped scaffolds were assessed because bifurcated scaffolds had not undergone validation testing and because the FDA has recommended testing coronary stents in simulated vessels with worst-case angulations and the American College of Cardiology and the American Heart Association do not define the S-shaped scaffold geometry as "angulated" $[3,40]$. The hypothesis in this case was that, after 1 day of cultivation of complex-shaped BVMs, no statistically significant differences would exist between the cell densities along the length of the BVMs, between the cell densities in the inner and outer halves of the BVMs, or between the cell densities in the top and bottom halves of the BVMs. 


\subsection{Materials and Methods}

3 L-shaped BVMs and 3 U-shaped BVMs were tissue-engineered, using laboratory techniques that will be detailed in this section. The laboratory techniques were carried out over a 2-week period, and a protocol that identifies when the laboratory techniques were used is located in Appendix H. The density of cells in various regions throughout each BVM was quantified, and it was determined whether cell densities were statistically significantly different along the length of each scaffold geometry, between the inner and outer halves of each scaffold geometry, and between the top and bottom halves of each scaffold geometry.

\subsubsection{Cell Culture}

HUVECs (Lonza) were cultured and expanded in T75 and T225 BD cell culture flasks. Cells were cultured in HUVEC media; the components of the HUVEC media and steps that were carried out to make the media are located in Appendix C. The cells were maintained at $37^{\circ} \mathrm{C}$ and $5 \% \mathrm{CO}_{2}$ in a small incubator with $80-100 \%$ humidity (Shellab $\mathrm{CO}_{2}$ Series Incubator, Sheldon Manufacturing, Inc.). During each passage from one flask to another flask, cells were monitored using an inverted light microscope (Olympus CKX31 with IX2-SLP contrast slider). When cells were pressure-sodded onto scaffolds, cells were no more than P8. Enough cells were cultured so that approximately 1.5 million cells $/ \mathrm{cm}^{2}$ were sodded onto each scaffold. 


\subsubsection{Sterilization and Media Preparation}

All scaffolds were made of 4-mm-ID ePTFE. 3 straight pieces of ePTFE were cut to lengths of $5 \mathrm{~cm}$ to serve as L-shaped scaffolds, and 3 straight pieces of ePTFE were cut to lengths of $5.5 \mathrm{~cm}$ to serve as U-shaped scaffolds. Female luer thread barbed fittings were inserted into both ends of each scaffold, and both ends of each scaffold were sutured onto the fittings using 2.0 silk suture as described in Section 2.3.4. The scaffolds were then placed in sterilization pouches, were sterilized in an autoclave for 30 minutes at $250^{\circ} \mathrm{C}$, and were then dried for 30 minutes. 3 forceps and a degassing flask with a rubber stopper were also sterilized in the autoclave. 3 luer plugs and 2 rotating male-to-male luer lock connectors were placed loose inside each of the polypropylene containers of 6 multifunctional bioreactors. The bioreactors with their loose fittings, 6 lids for each of the bioreactors, and 6 two-port lids for media reservoirs were sterilized with ethylene oxide gas (EtO) at the Cal Poly Veterinary Clinic.

Throughout the tissue engineering of straight BVMs, three types of media are used, and these three types of media were also used for L- and U-shaped BVMs in this study. The three types of media are bioreactor media, conditioning media, and HUVEC media. 2 bottles of bioreactor media were made for use in this study, 1 bottle of conditioning media was made for use in this study, and 1 bottle of HUVEC media in addition to the bottle used during cell culture was made for use in this study. The components included in each type of media and the steps that were carried out to make each type of media are located in Appendices C, D, and E. 


\subsubsection{Denucleation and Protein-Coating of Scaffolds}

All 6 scaffolds were denucleated and coated with protein. First, $60 \mathrm{~mL}$ of conditioning media was warmed in a water bath at $37^{\circ} \mathrm{C}$, placed in the sterilized degassing flask, and placed under vacuum. $615-\mathrm{mL}$ conicals were each filled with $10 \mathrm{~mL}$ of sterile $70 \%$ ethanol (EtOH), and each scaffold was placed fully submerged in one of the $15-\mathrm{mL}$ conicals for 10 minutes. During the first 30 seconds of submersion, the conicals were tapped to dislodge air bubbles on the scaffolds. 6 other $15-\mathrm{mL}$ conicals were each filled with $10 \mathrm{~mL}$ of sterile $100 \% \mathrm{EtOH}$, and each scaffold was moved from the $70 \% \mathrm{EtOH}$ directly into the $100 \% \mathrm{EtOH}$ using forceps. The scaffolds were fully submerged again, and the conicals were tapped again during the first 30 seconds of submersion to dislodge air bubbles on the scaffolds. 6 other 15-mL conicals were each filled with $10 \mathrm{~mL}$ of degassed conditioning media, and each scaffold was moved from the $100 \% \mathrm{EtOH}$ directly into the degassed conditioning media; scaffolds were gently submerged in the degassed conditioning media to prevent formation of air bubbles during submersion. The scaffolds were placed in an incubator at $37^{\circ} \mathrm{C}$ overnight.

\subsubsection{Insertion of Scaffolds into Bioreactors and Priming}

All media was warmed in a water bath at $37^{\circ} \mathrm{C}$. The 6 sterilized multifunctional bioreactors, the 6 sterilized lids for each bioreactor, the 6 sterilized two-port lids for media reservoirs, and 2 sterilized forceps were gathered. The $615-\mathrm{mL}$ conicals, containing 6 scaffolds that had been denucleated and protein-coated, were placed inside a laminar flow hood (Nuaire Labgard Class II Type A2 Biological Safety Cabinet). Two sterile bioreactors were placed inside the laminar flow hood, and approximately $200 \mathrm{~mL}$ 
of bioreactor media were poured into both bioreactors. Forceps were used to transfer each scaffold out of its conical and into a bioreactor. Sterile gloves were then donned, the loose luer plugs were secured into position to cap off any inlets or outlets that would not be used when a U-shaped scaffold was inserted, rotating male-to-male luer lock connectors were secured into position for a U-shaped scaffold, and the scaffold was secured into position to form a U-shape (Figure 3.3). Securement of luer plugs, rotating male-to-male luer lock connectors, and U-shaped scaffold was also carried out in the other bioreactor. Lids were then placed on both bioreactors, and sterile gloves were removed.

The scaffolds were then primed with conditioning media. A syringe was filled with $10 \mathrm{~mL}$ of conditioning media and attached to the inlet of the U-shaped scaffold on one of the bioreactors. The syringe was used to inject $5 \mathrm{~mL}$ of conditioning media into the lumen of the scaffold, removing all air bubbles from the lumen. The luminal outlet of the scaffold was then closed off, and the remaining $5 \mathrm{~mL}$ of conditioning media in the syringe were forced into the lumen of the scaffold but also through the pores of the scaffold. These priming steps were repeated for the U-shaped scaffold in the other bioreactor. 


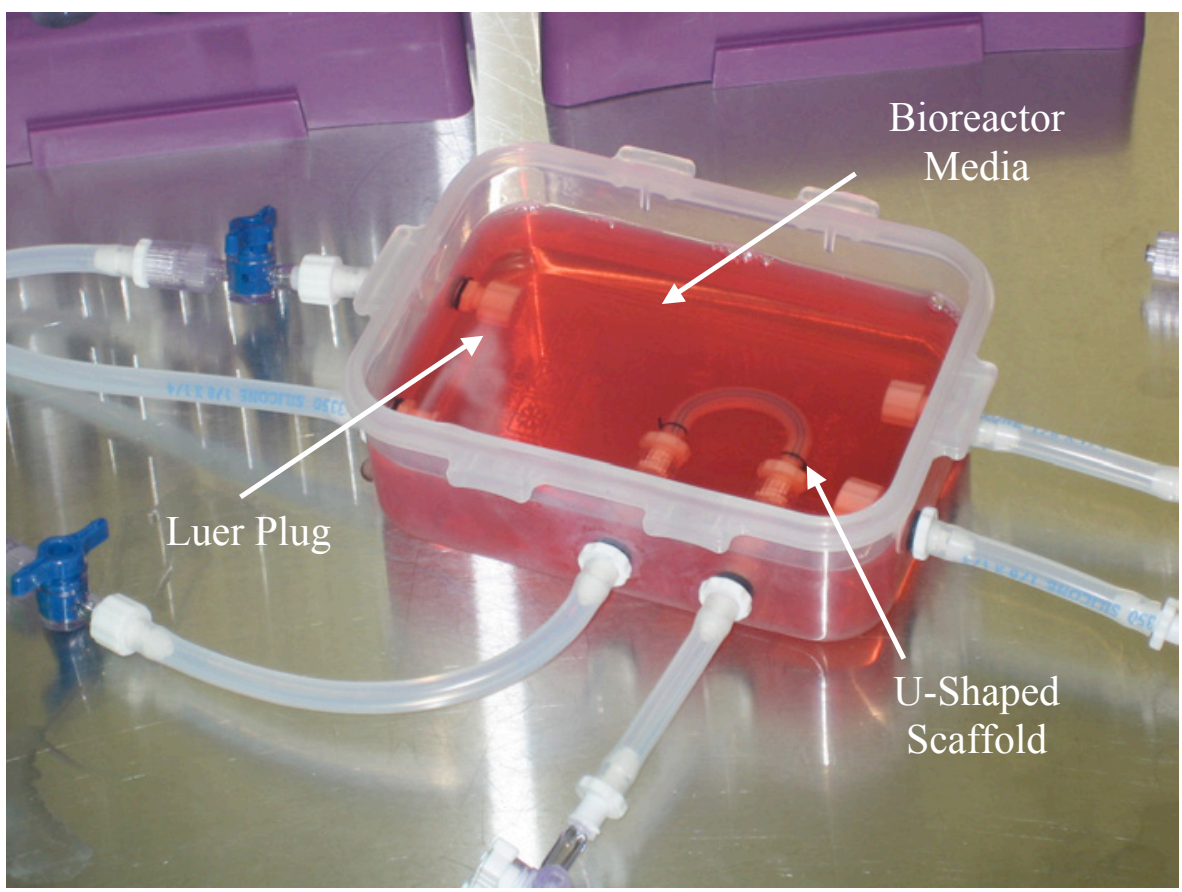

Figure 3.3. Insertion of U-shaped scaffold into bioreactor. All fittings were secured into place, and the scaffold was secured in a U-shape. All scaffolds were positioned with the blue double stripe upward (see double blue stripe on top of scaffold in image).

The reservoirs with two-port lids were then primed. $45 \mathrm{~mL}$ of conditioning media were placed into a 50-mL conical for each reservoir. Two-port lids were then attached to each conical, and the conicals were inverted so that media flowed through the tubing attached to the conicals, removing all air from inside the tubing. Each two-port lid was then connected to one of the bioreactors so that the inlet and outlet of the two-port lids were correctly connected to the inlet and outlet of the bioreactors (Figure 3.4).

The bioreactors and their respective two-port lids were placed in a large incubator (Shellab $\mathrm{CO}_{2}$ Incubator) at $37^{\circ} \mathrm{C}$ and $5 \% \mathrm{CO}_{2}$ and attached to a peristaltic pump (ColeParmer Masterflex L/S Model 8-Roller) (Figure 3.5). First, the luminal outlets and luminal inlets of both bioreactors were opened so that media could be pumped through 
the complex-shaped BVM system to remove any remaining air bubbles from the systems. The pump was turned on to $10 \mathrm{rpm}$, which corresponds to $0.8 \mathrm{~mL} / \mathrm{min}$, media was pumped through the systems, and all air bubbles were removed from the systems. The luminal outlet was then closed, and media was pumped transmurally through the systems for 10 minutes at $150 \mathrm{rpm}$, which corresponds to $12 \mathrm{~mL} / \mathrm{min}$. All inlets and outlets were then closed to prevent media backflow. The two systems were left in the large incubator.

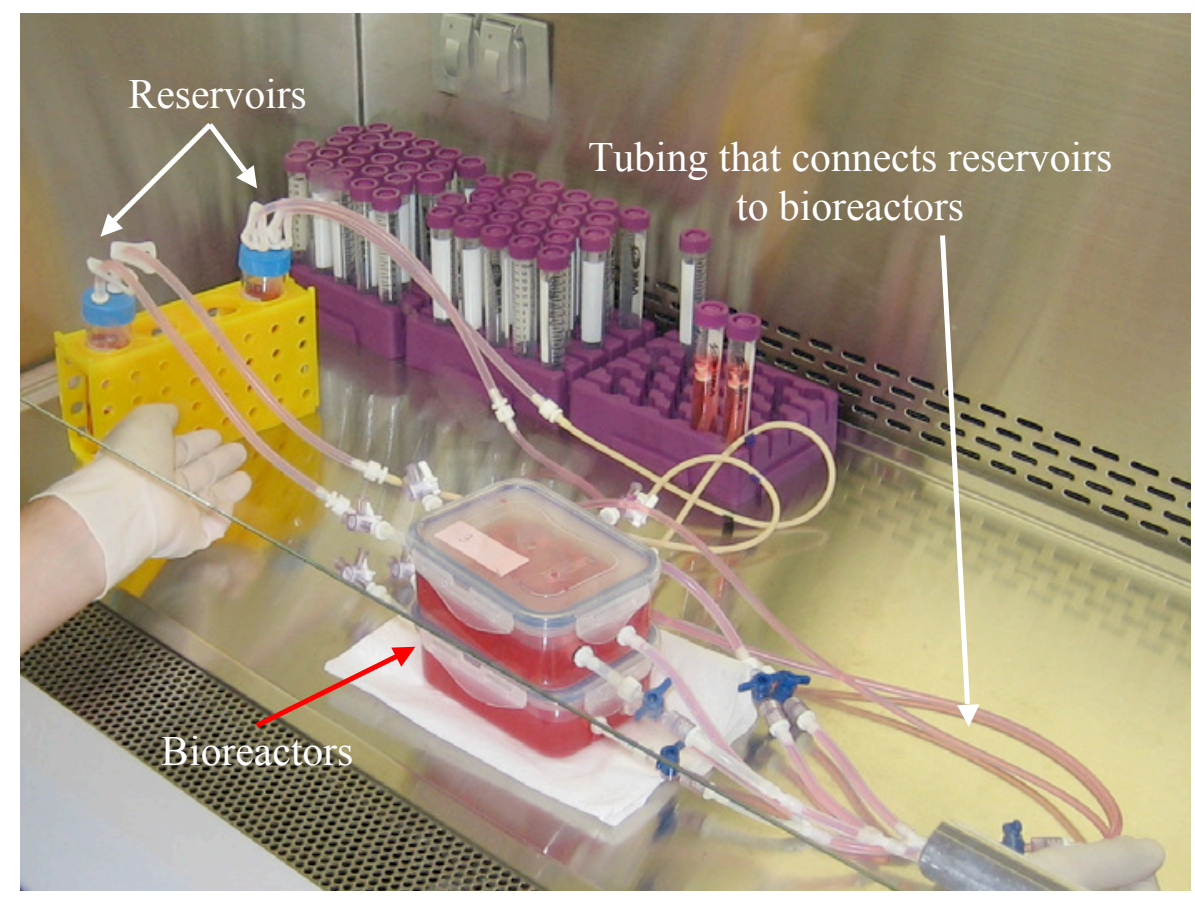

Figure 3.4. Media reservoirs. After air bubbles are removed from the reservoir tubing, the tubing is connected to a bioreactor. 


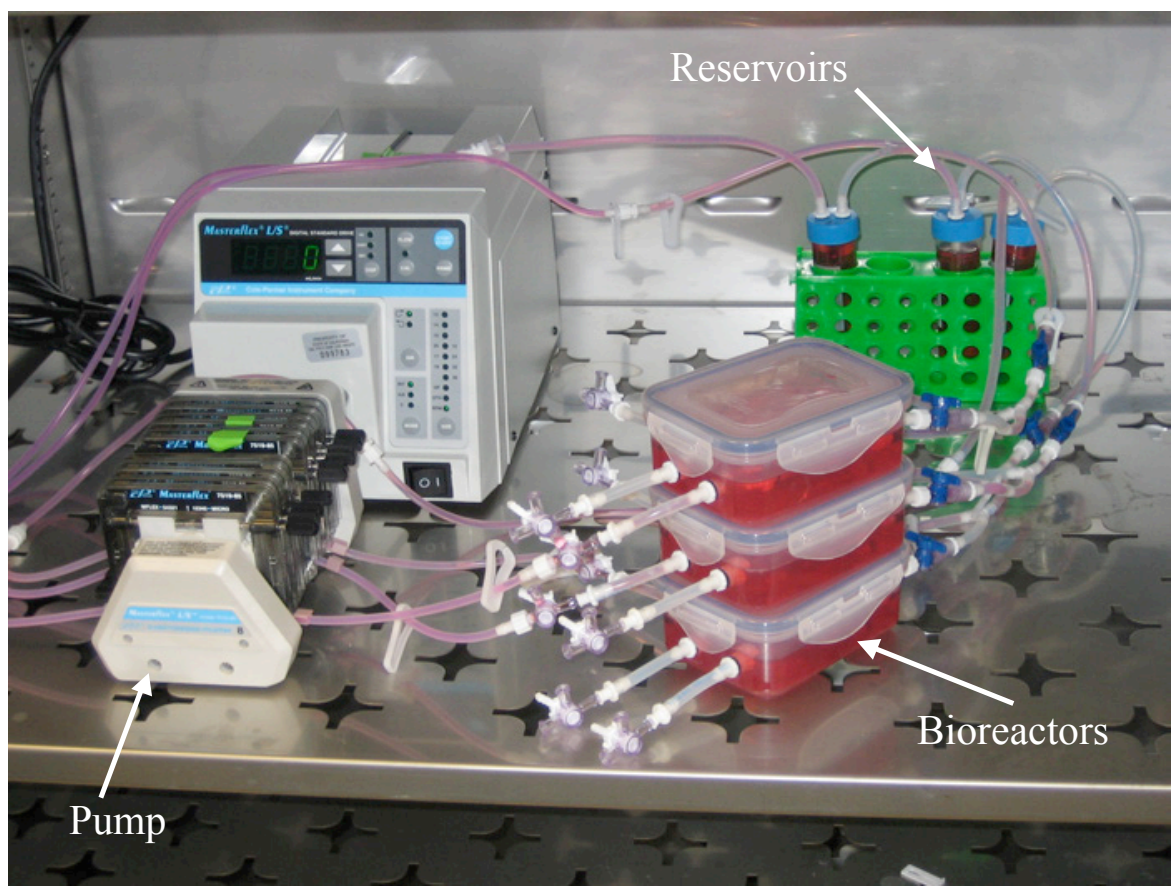

Figure 3.5. Complex-shaped BVM systems connected to a pump. The pump circulates media through the 3 complex-shaped BVM systems shown here. Media flows from the reservoirs to the pump to the bioreactors.

These steps were repeated with two more bioreactors, one bioreactor housing a Ushaped scaffold and the other bioreactor housing an L-shaped scaffold. These steps were then repeated a third time with the last two bioreactors, both bioreactors housing Lshaped scaffolds. Completion of all the steps resulted in 6 primed complex-shaped BVM systems: 3 complex-shaped BVM systems containing U-shaped scaffolds and 3 complexshaped BVM systems containing L-shaped scaffolds.

\subsubsection{Pressure-Sodding of HUVECs}

Cell culture was carried out so that 6 confluent T225 flasks would be available on the day that cells were sodded onto the scaffolds. 1 T225 flask of HUVECs was allotted 
for each scaffold. After all 6 complex-shaped BVM systems were primed, the 6 flasks were removed from the small incubator, and the cells in each flask were inspected. Cells were removed from the bottom of each flask using trypsin. The suspensions of cells in each flask were transferred to their own 50-mL conicals. Each conical was centrifuged on setting 4 for 4 minutes to pellet all cells at the bottom of each conical.

After a pellet of cells formed, the conical was taken to the laminar flow hood, and the supernatant was aspirated out of the conical without aspirating out the pellet of cells. The cells were resuspended in $4 \mathrm{~mL}$ of HUVEC media. A complex-shaped BVM system was then brought out of the large incubator and into the laminar flow hood. The reservoir was placed to the side in the hood so that the bioreactor could be easily accessible during pressure-sodding. The bioreactor outlet was disconnected from the tubing that leads to the reservoir and was placed into a media trough so that media could flow out of the bioreactor outlet and into the media trough. The luminal outlet was closed, and the extraluminal outlet was opened. Two other troughs were prepared: one trough containing $10 \mathrm{~mL}$ of HUVEC media and another trough containing the $4 \mathrm{~mL}$ of cell suspension. A syringe was filled with $5 \mathrm{~mL}$ of HUVEC media and attached to the stopcock proximal to the scaffold, and all $5 \mathrm{~mL}$ of HUVEC media in the syringe were injected into the tubing proximal to the scaffold to remove conditioning media from the proximal tubing. Then another syringe was filled with the $4 \mathrm{~mL}$ of cell suspension, the syringe was attached to the stopcock proximal to the scaffold, and all $4 \mathrm{~mL}$ of cell suspension were injected transmurally into the scaffold. The syringe used to previously inject HUVEC media was used to inject the remaining HUVEC media transmurally into the scaffold. Then all inlets and outlets were closed to prevent media backflow, and the bioreactor outlet was 
reconnected to the reservoir tubing. The reservoir containing conditioning media was replaced with a reservoir containing HUVEC media. The complex-shaped BVM system was reattached to the pump in the large incubator, and the pump was immediately turned on to a setting of $10 \mathrm{rpm}(0.8 \mathrm{~mL} / \mathrm{min})$ and administered transmural flow.

These pressure-sodding steps were repeated for each of the 6 scaffolds.

\subsubsection{Cultivation}

Transmural flow at $10 \mathrm{rpm}(0.8 \mathrm{~mL} / \mathrm{min})$ was maintained on all complex-shaped BVMs for 1 hour. Then the luminal outlet on all bioreactors was opened, and luminal flow at $10 \mathrm{rpm}(0.8 \mathrm{~mL} / \mathrm{min})$ was maintained for another hour. Luminal flow was then increased to $15 \mathrm{rpm}(1.2 \mathrm{~mL} / \mathrm{min})$ and maintained overnight until the complex-shaped BVMs were harvested the next day.

\subsubsection{Harvesting and Fixation}

After 1 day of cultivation, all complex-shaped BVMs were harvested from their bioreactors. First, all inlets and outlets on each bioreactor were closed off. Then the pump was turned off. All bioreactors were removed from the large incubator to a nonsterile surface. Six 15-mL conicals were each filled with $8 \mathrm{~mL}$ of Histochoice and labeled for a specific complex-shaped BVM; the bottom end of each conical was marked "Proximal," and the top end of each conical was marked "Distal" to keep track of proximal and distal ends of each complex-shaped BVM. The lid of each bioreactor was removed, and a razor blade was used to cut the ends of each complex-shaped BVM off the female luer thread barbed fittings, making sure to not do anything that could disrupt the cell lining of each 
BVM. Forceps were used to transfer each complex-shaped BVM into the corresponding 15-mL conical of Histochoice, orienting each complex-shaped BVM to align with the orientation markings on the outside of each conical. All complex-shaped BVMs remained in Histochoice overnight to ensure that fixation occurred. All media in each bioreactor and reservoir was disposed of, residual ePTFE scraps on the female luer thread barbed fittings in each bioreactor were disposed of, and all bioreactors, bioreactor lids, two-port lids, and tubing were cleaned with hot water, dried, and stored for future experiments.

\subsubsection{BBI Imaging}

After 1 day of fixation in Histochoice, all complex-shaped BVMs were cut into smaller pieces for analysis of cell deposition in various regions. First, each BVM was cut into 5 segments (Figure 3.6 A and B). Recall that during scaffold insertion into the bioreactors, the blue double stripe on each piece of ePTFE was positioned upward. Each scaffold segment was cut in half longitudinally so that each half had one of the blue lines that originally formed the double blue stripe. Enough $15-\mathrm{mL}$ conicals were wrapped in foil so that each cut segment was individually stored in its own conical; each conical was labeled with information that identified the specific segment that would be stored in that conical (e.g. "L-Shaped BVM \#1, $\mathrm{P}_{1}$, inner"). A BBI solution was prepared as described in Appendix G, $1 \mathrm{~mL}$ of BBI solution was placed in each conical, and 1 scaffold segment was placed in each conical. En face BBI images were taken of each scaffold segment on a fluorescent microscope (Olympus BX41) using the protocol included in Appendix G. 4 images were taken of each scaffold segment: 2 images on the top half of each segment and two images on the bottom half of each segment (Figure 3.6 C). 


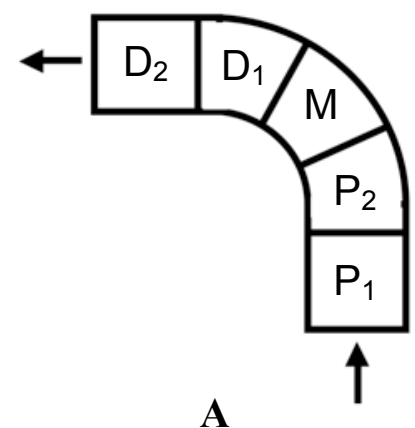

A

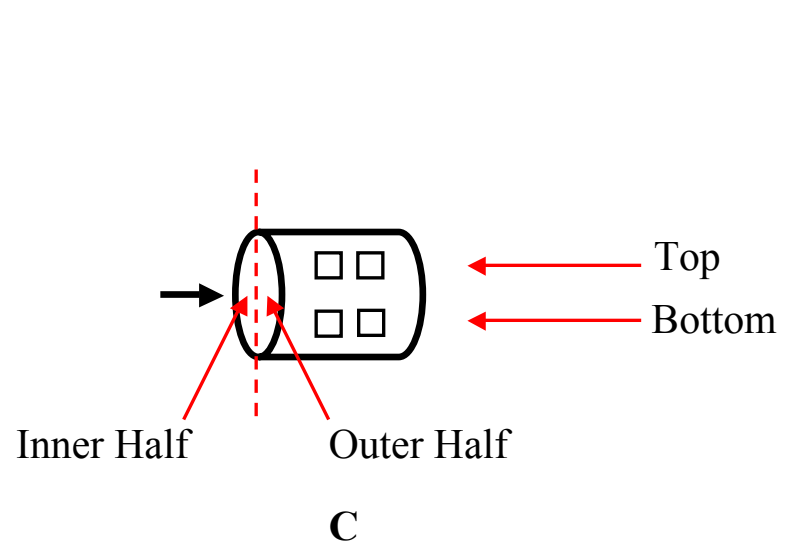

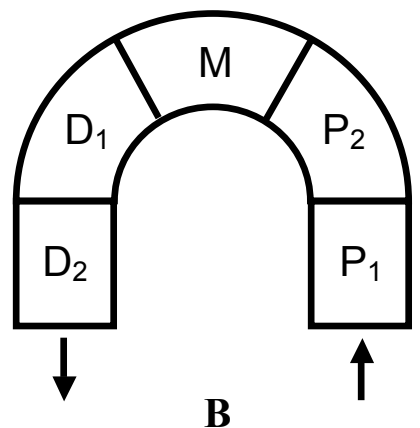

B

Figure 3.6. Cutting of L- and U-shaped BVMs into segments for evaluation. L- and Ushaped BVMs were first cut into 5 segments: Proximal $1\left(\mathrm{P}_{1}\right)$, Proximal $2\left(\mathrm{P}_{2}\right)$, Middle $(\mathrm{M})$, Distal $1\left(\mathrm{D}_{1}\right)$, and Distal $2\left(\mathrm{D}_{2}\right)$; black arrows indicate the direction of flow $(\mathrm{A}$ and B). C shows an alternate view of segment $\mathrm{P}_{1}$ from both scaffold geometries; the black arrow indicates the direction of flow, the vertical red line indicates the demarcation between inner and outer halves of the BVMs, and the 4 black boxes indicate the location of 4 BBI images that were captured on each BVM segment. Because 6 complex-shaped BVMs were tissue-engineered for this study, and because each BVM was cut into 10 segments, there were a total of 60 scaffold segments that were evaluated. Because 4 BBI images were captured on each segment, a total of $240 \mathrm{BBI}$ images were captured for this study. 


\subsubsection{Cell Counting in BBI Images}

After BBI images were obtained for all segments of all complex-shaped BVMs, cells were counted in the images. Instead of counting all cells in every image, a template was applied to every image that highlighted the same small 5 regions in each image (Figure 3.7). One person counted cells in these regions by identifying cells with the naked eye and keeping a tally of the number of cells that were identified.

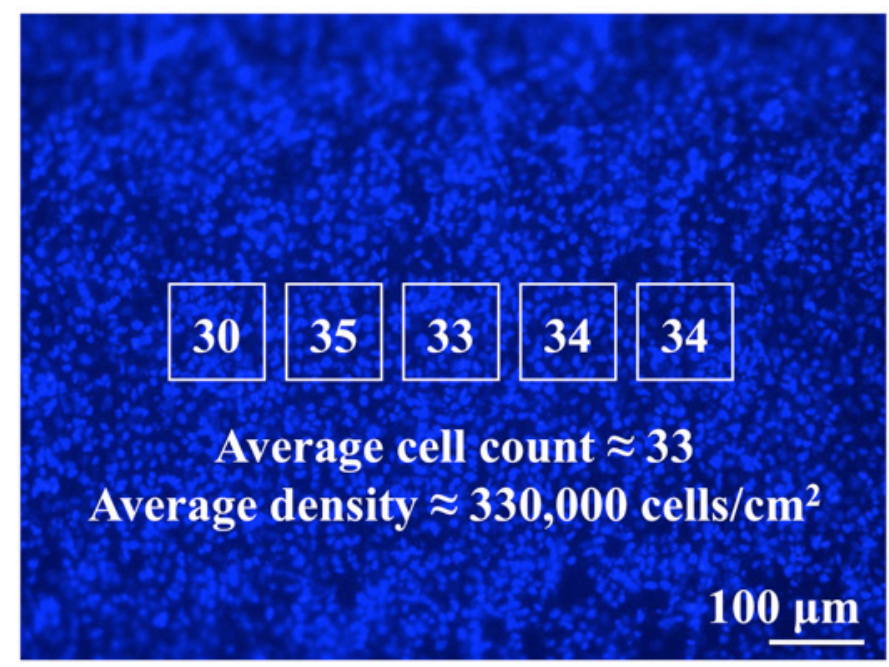

Figure 3.7. Cell counting in BBI images. 5 small white boxes were placed across the center of each image; each small white box has an area of $10,000 \mu \mathrm{m}^{2}$. Each blue dot represents the nucleus of a cell, and the blue dots were counted in each small white box. Note that cells in the top edge of the image are blurry; no small white boxes contained any blurry cells, and no blurry cells were counted. The counts from the small white boxes were averaged to yield a representative count for the image, and the average was converted to a representative density in units of cells $/ \mathrm{cm}^{2}$. 
Just before images were taken of each scaffold segment, the edge of each scaffold segment was blotted on a Kimwipe to wick residual BBI solution from the luminal surface of the scaffold segments; this method was repeatedly used with any scaffold segments whose blue dots were difficult to visualize on the microscope. Even after repeated blotting with a Kimwipe, the blue dots in 20 images taken on the outer half of one of the U-shaped BVMs were still difficult to visualize. Therefore, cells were not counted in those 20 images. Of the total 240 images that were captured in this study, cells were counted in 220 images, and the 20 images taken on that outer half of one of the Ushaped BVMs were not included in the following statistical analysis.

\subsubsection{Statistical Analysis of BBI Images}

After representative cell densities were determined for the 220 BBI images, the cell densities in different regions of the complex-shaped BVMs were compared to each other to determine whether any cell densities were statistically significantly different from each other. The number of images taken for L-shaped BVMs totaled to 120, and the number of images taken for U-shaped BVMs totaled to 100. Since a representative cell density was associated with each image, the number of representative cell densities for Lshaped BVMs totaled to 120 , and the number of representative cell densities for Ushaped BVMs totaled to 100. To determine if the data set for L-shaped BVMs and the data set for U-shaped BVMs were each normally distributed, normal probability plots were generated for both data sets using Minitab 16.1.1. If a data set was normally distributed, a one-way analysis of variance (ANOVA) was used to determine whether statistically significant differences existed between the cell densities in various regions of 
the scaffold. If a data set was not normally distributed, the Kruskal-Wallis test was used to determine whether statistically significant differences existed between the cell densities in various regions of the scaffold. Boxplots and individual value plots were created so that the differences between cell densities in various regions of each scaffold could be better visualized.

\subsection{Results}

A sample BBI image for each of the 6 complex-shaped BVMs is shown in Figure 3.8, and some representative cell counts are summarized in Table 3.1. Appendix I includes additional BBI images, the representative cell counts that were established for all $220 \mathrm{BBI}$ images, and the method used to convert representative cell counts into cell densities with units of cells $/ \mathrm{cm}^{2}$. 

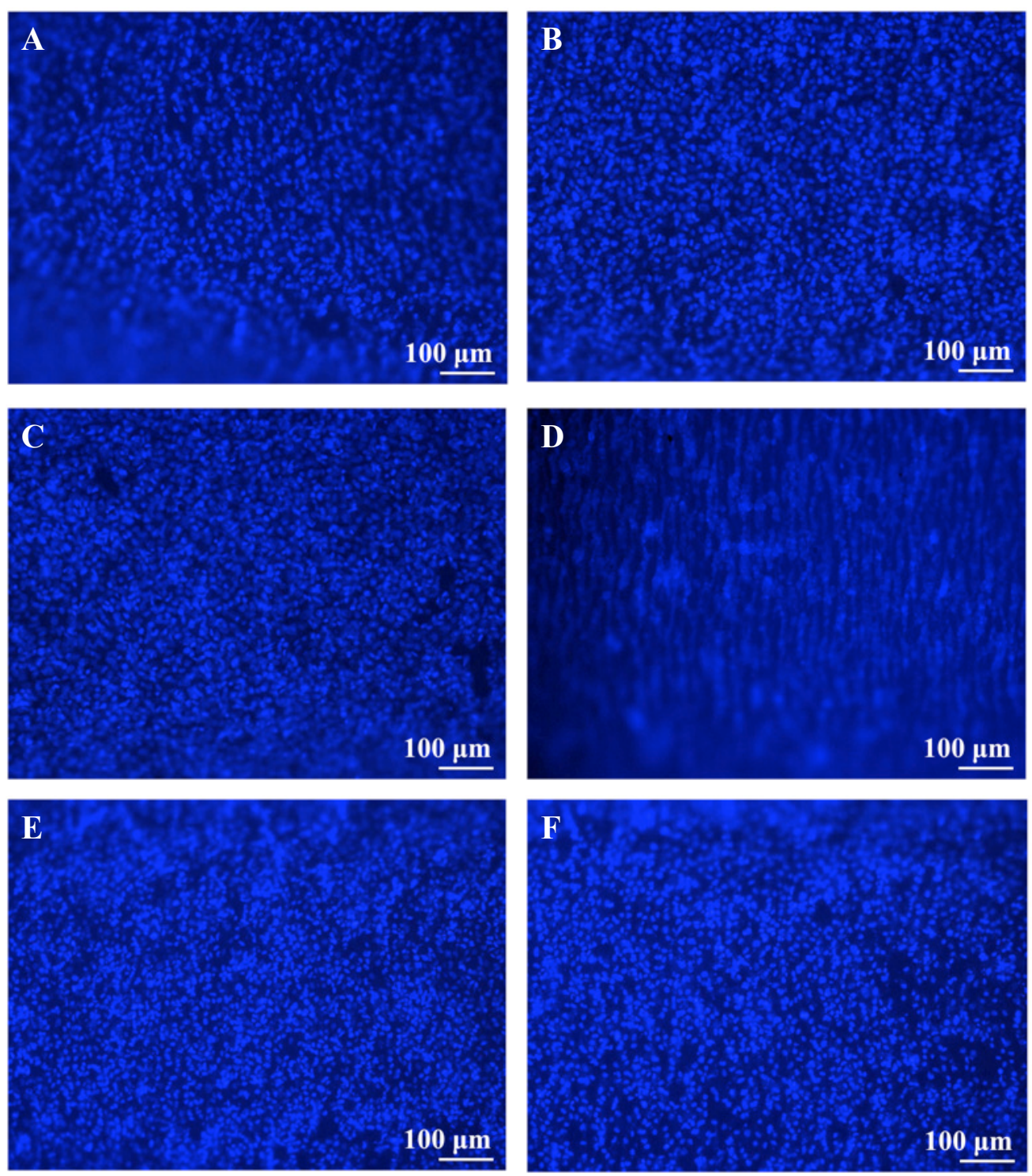

Figure 3.8. Sample BBI images from the 6 complex-shaped BVMs in this study. A sample BBI image is shown for each of the three L-shaped BVMs $(\mathrm{A}-\mathrm{C})$ and each of the three U-shaped BVMs (D-F). D shows blue splotches instead of blue dots.

Table 3.1. Average Cell Counts \pm SD for an Area of $10,000 \mu \mathrm{m}^{2}$ Along Length of BVMs

\begin{tabular}{|c|c|c|c|c|c|}
\hline BVM & Distal 2 & Distal 1 & Middle & Proximal 2 & Proximal 1 \\
\hline L-shaped & $31 \pm 2$ & $33 \pm 2$ & $33 \pm 3$ & $32 \pm 2$ & $30 \pm 2$ \\
\hline U-shaped & $27 \pm 4$ & $30 \pm 4$ & $30 \pm 3$ & $29 \pm 3$ & $26 \pm 5$ \\
\hline
\end{tabular}


Almost all BBI images showed blue dots whose edges could be clearly identified. But the $20 \mathrm{BBI}$ images taken on the outer half of one of the U-shaped BVMs showed blue splotches instead of blue dots (Figure 3.8 D), and the edges of the splotches were much less identifiable than the edges of the dots, therefore those 20 images were not quantified or included in any statistical analyses.

The normal probability plots showed that the data set for L-shaped BVMs was not normal and that the data set for U-shaped BVMs was normal (Figure 3.9). Because the data set for L-shaped BVMs was not normal, the Kruskal-Wallis test was used to determine whether statistically significant differences existed between the cell densities in various regions of L-shaped scaffolds. Because the data set for U-shaped BVMs was normal, a one-way ANOVA was used to determine whether statistically significant differences existed between the cell densities in various regions of U-shaped scaffolds. 

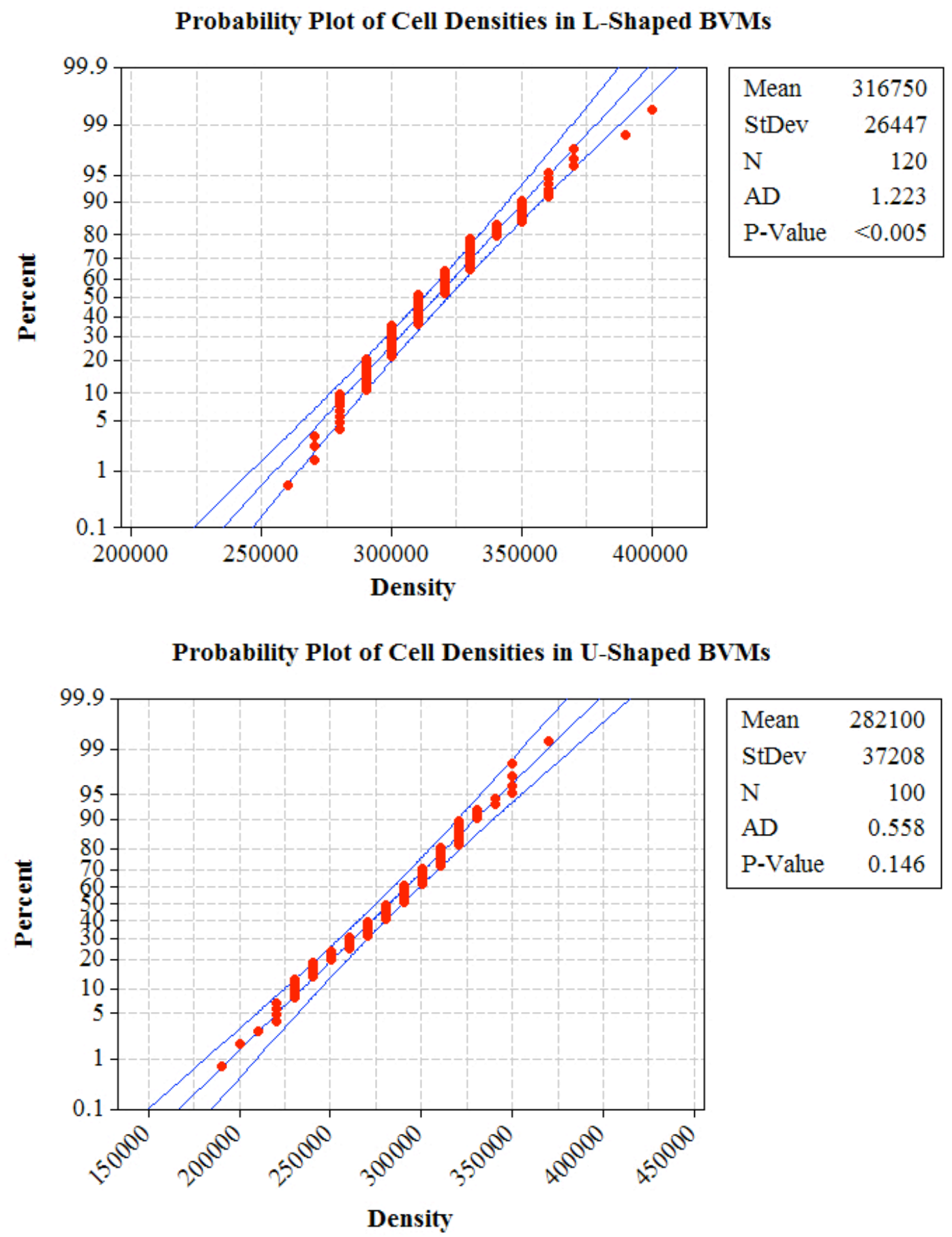

Figure 3.9. Probability plots of data sets for L- and U-shaped BVMs. A 95\% confidence interval was used. A normal data set has all data points inside the outer blue lines and has $p>0.05$. The L-shaped BVM data set had $p<0.005$ and was therefore not normal. The U-shaped BVM data set had $\mathrm{p}=0.146$ and was therefore normal. 
For L-shaped BVMs, there was a statistically significant difference between the cell densities of the 5 regions along the length of the BVMs, there was a statistically significant difference between the cell densities in the inner and outer halves of the BVMs, and there was no statistically significant difference between the cell densities in the top and bottom halves of the BVMs. For U-shaped BVMs, there was a statistically significant difference between the cell densities in the 5 regions along the length of the BVMs, there was no statistically significant difference between the cell densities in the inner and outer halves of the BVMs, and there was no statistically significant difference between the cell densities in the top and bottom halves of the BVMs. Table 3.2 shows the $\mathrm{p}$-values for each statistical comparison; when $\mathrm{p}<0.05$, a statistically significant difference existed. Appendix J contains the Minitab output that shows each p-value.

Table 3.2. P-Values for All Statistical Comparisons

\begin{tabular}{|c|c|c|}
\hline Comparison & $\begin{array}{c}\text { L-Shaped } \\
\text { BVM }\end{array}$ & $\begin{array}{c}\text { U-Shaped } \\
\text { BVM }\end{array}$ \\
\hline $\begin{array}{c}\text { 5 Regions Along } \\
\text { Length of BVM }\end{array}$ & 0.000 & 0.008 \\
\hline Inner vs. Outer & 0.011 & 0.057 \\
\hline Top vs. Bottom & 0.607 & 0.810 \\
\hline
\end{tabular}

Boxplots allowed for better visualization of the differences between cell densities in various regions of the complex-shaped BVMs. While the one-way ANOVA compared averages in the U-shaped BVM data set, the Kruskal-Wallis test compared medians in the L-shaped BVM data set. Therefore, U-shaped BVM boxplots were constructed based on 
averages, while L-shaped BVM boxplots were constructed based on medians. In some cases, individual value plots were constructed to show the specific data points that were used to construct boxplots. Although the cell densities were statistically significantly different between the 5 regions along the length of both L- and U-shaped BVMs and between the inner and outer halves of L-shaped BVMs, boxplots showed that all the median cell densities throughout L-shaped BVMs and all the average cell densities throughout U-shaped BVMs fell between $250,000 \mathrm{cells} / \mathrm{cm}^{2}$ and $350,000 \mathrm{cells} / \mathrm{cm}^{2}$ (Figures 3.10-3.17). 
Boxplot of Cell Density in Regions Along Length of L-Shaped BVMs

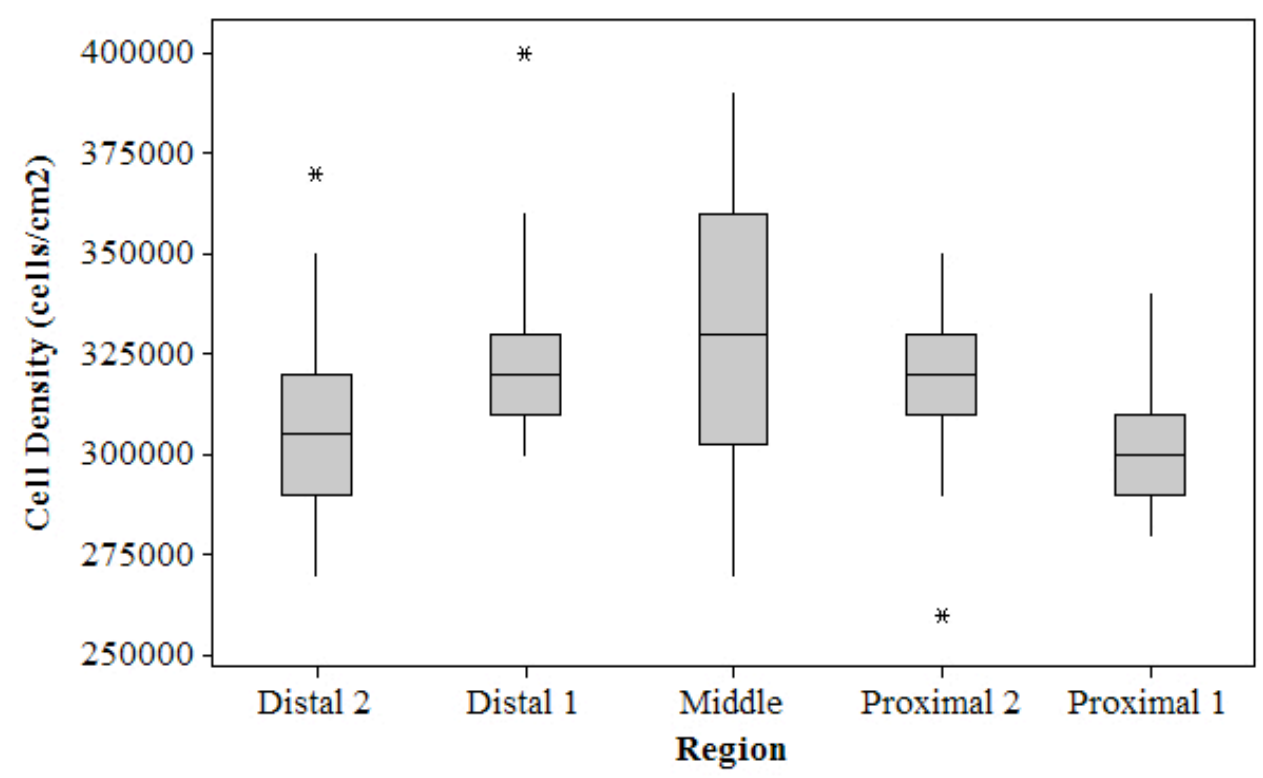

Figure 3.10. Boxplot of cell density along length of L-shaped BVMs. Asterisks mark outliers, and the center line of each structure corresponds to a median cell density.

Plot of Cell Density in Regions Along Length of 3 L-Shaped BVMs

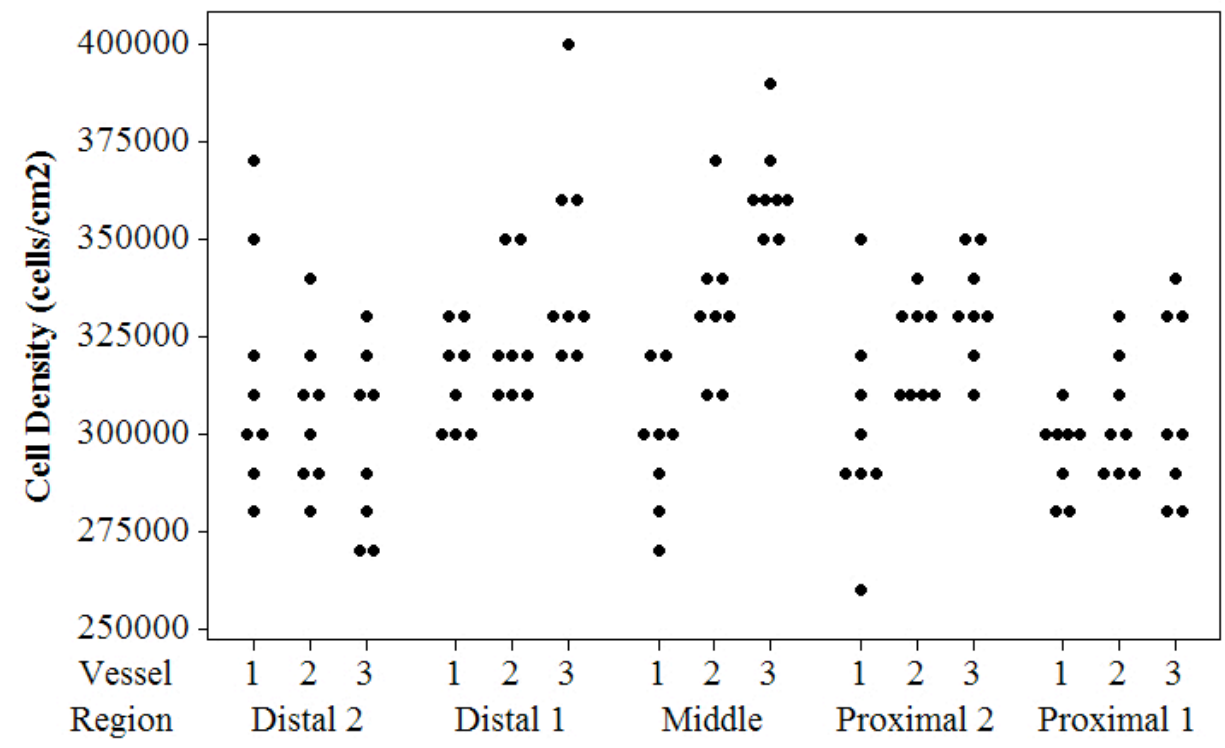

Figure 3.11. Individual value plot of cell density along length of L-shaped BVMs. The cell densities in this plot were used to create the boxplot in Figure 3.10. 


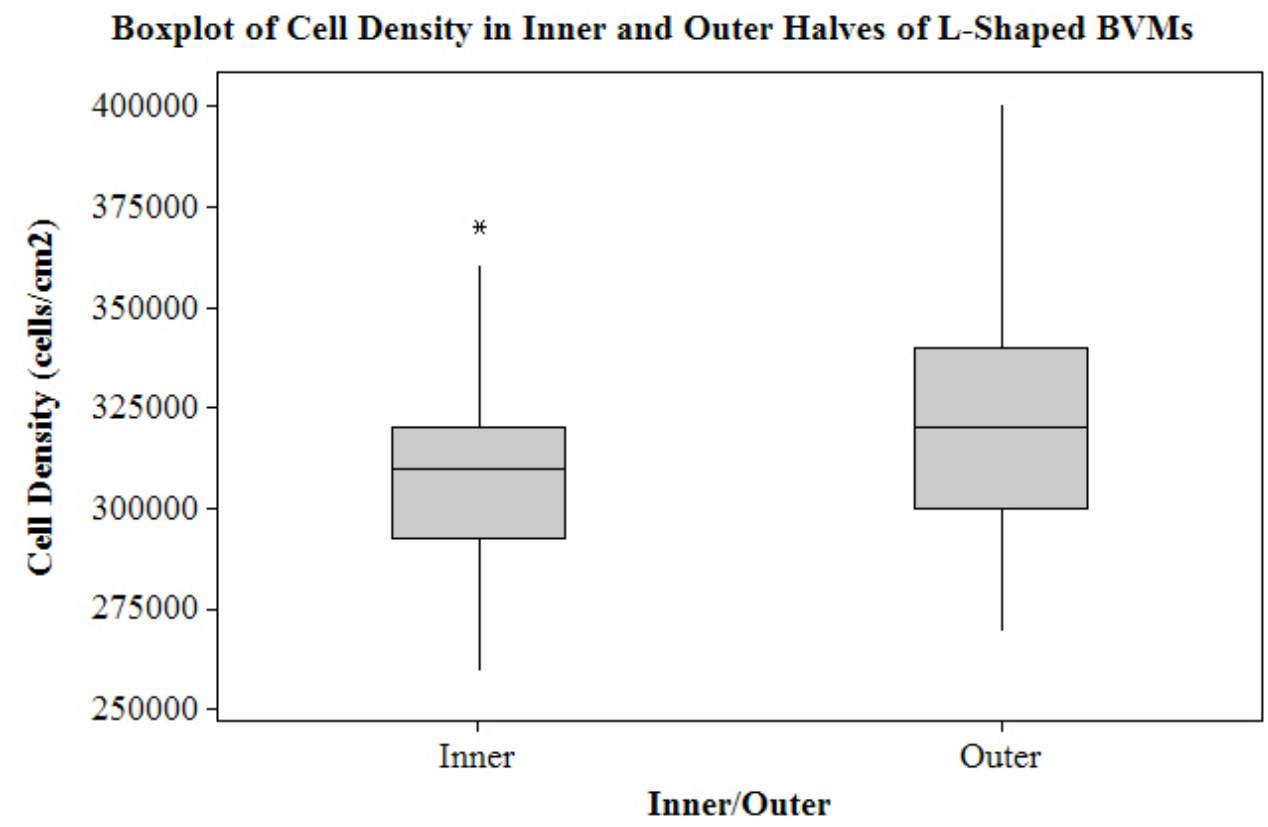

Figure 3.12. Boxplot of cell density in inner and outer halves of L-shaped BVMs. The asterisk marks an outlier, and the centerline of each structure corresponds to a median cell density.

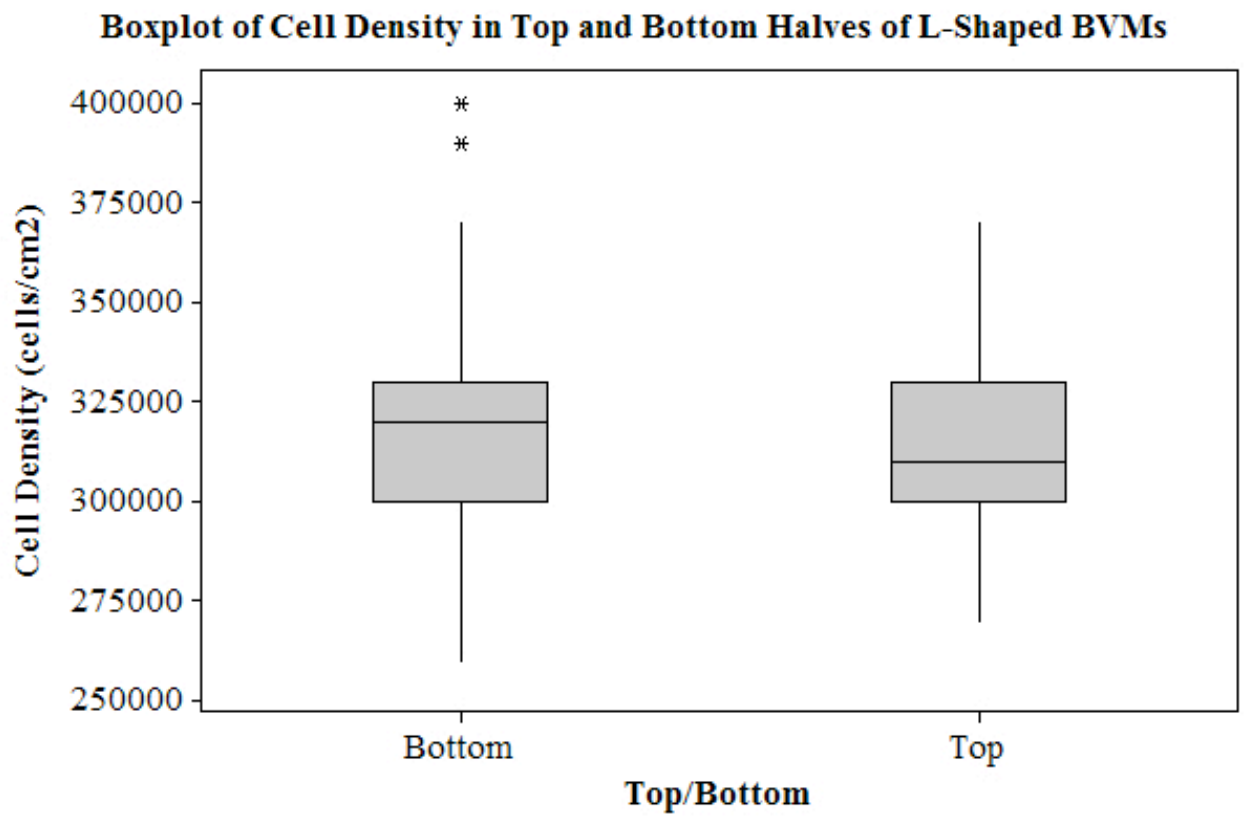

Figure 3.13. Boxplot of cell density in top and bottom halves of L-shaped BVMs. Asterisks mark outliers, and the center line of each structure corresponds to a median cell density. 


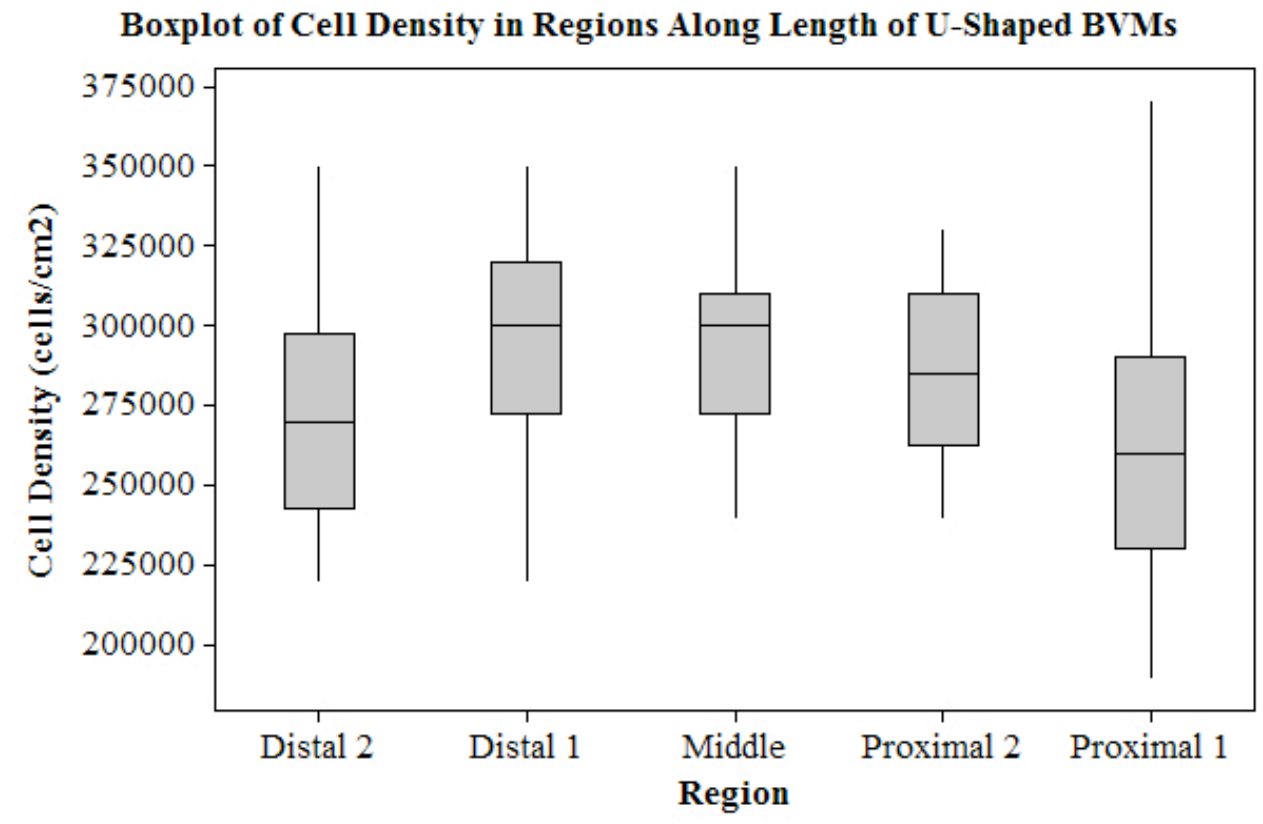

Figure 3.14. Boxplot of cell density along length of U-shaped BVMs. The center line of each structure corresponds to an average cell density.

Plot of Cell Density in Regions Along Length of 3 U-Shaped BVMs

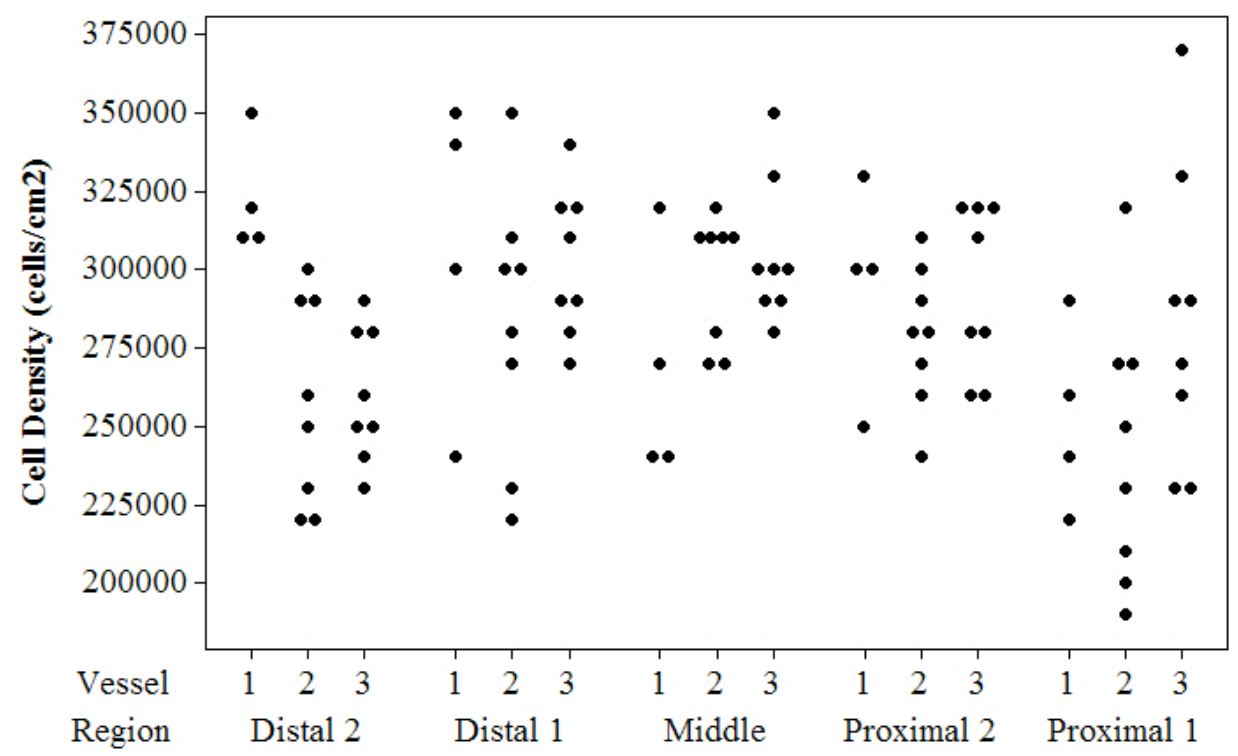

Figure 3.15. Individual value plot of cell density along length of U-shaped BVMs. The cell densities in this plot were used to create the boxplot in Figure 3.14. 
Boxplot of Cell Density in Inner and Outer Halves of U-Shaped BVMs

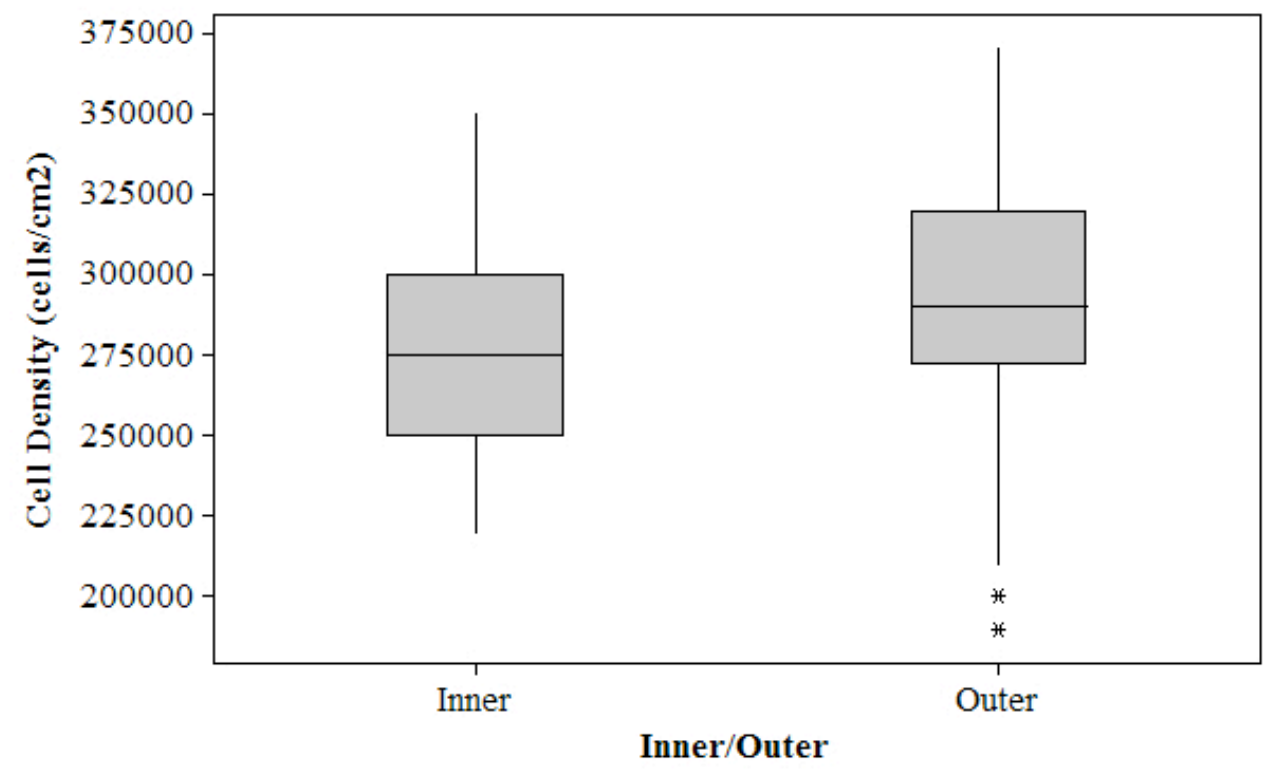

Figure 3.16. Boxplot of cell density in inner and outer halves of U-shaped BVMs. Asterisks mark outliers, and the center line of each structure corresponds to an average cell density.

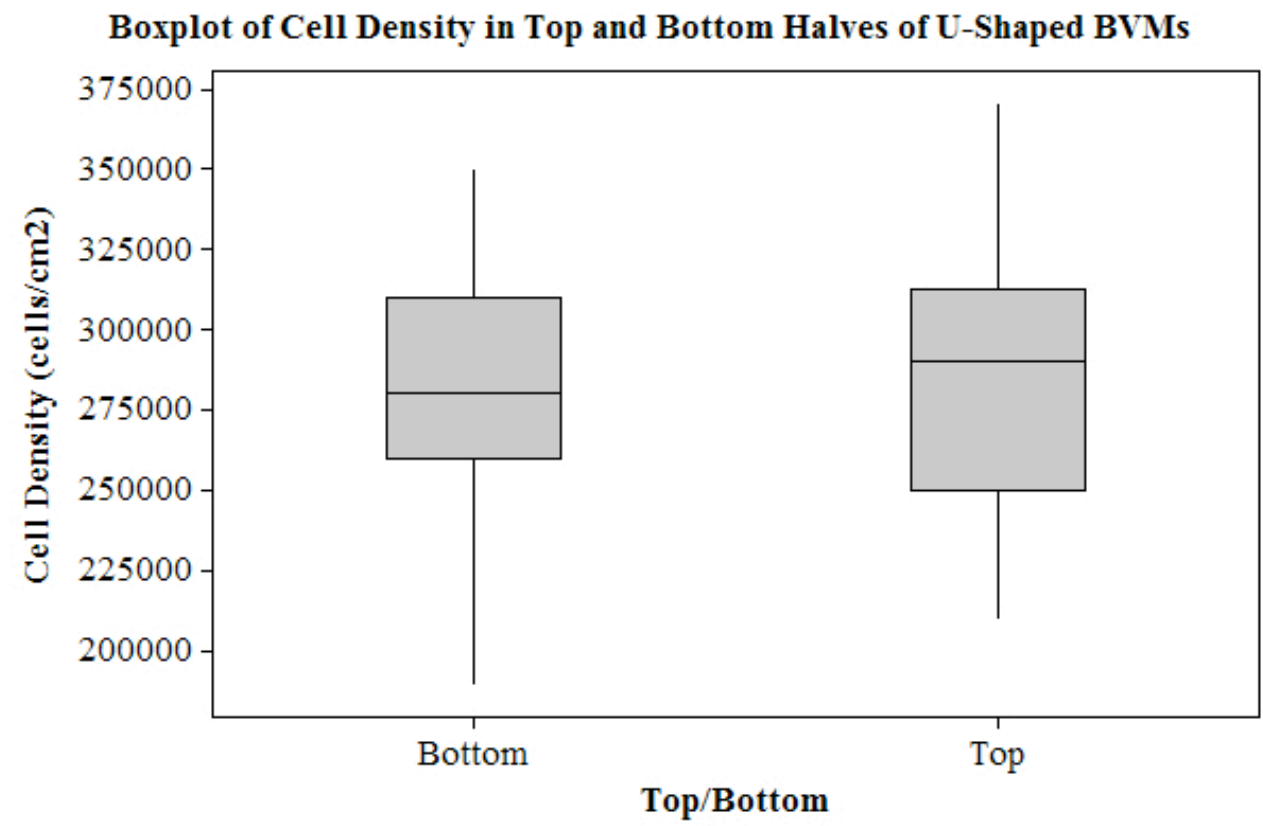

Figure 3.17. Boxplot of cell density in top and bottom halves of U-shaped BVMs. The center line of each structure corresponds to an average cell density. 


\subsection{Discussion and Conclusions}

Consistency of cell deposition throughout complex-shaped scaffolds was quantitatively assessed in this chapter. Consistent cell deposition may lead to development of a cell lining that is consistently confluent throughout BVMs, which may more accurately predict re-endothelialization of coronary stents. Cells were deposited onto L- and U-shaped scaffolds, and cell density after 1 day of cultivation was statistically significantly different between various regions in both L- and U-shaped BVMs. In L-shaped BVMs, cell density was statistically significantly different between 5 regions along the length of the BVMs and between the inner and outer halves of the BVMs. In U-shaped BVMs, cell density was statistically significantly different in 5 regions along the length of the BVMs. In other cases, cell density was not statistically significantly different. In L-shaped BVMs, cell density was not statistically significantly different between the top and bottom halves of the BVMs. In U-shaped BVMs, cell density was not statistically significantly different between the inner and outer halves of the BVMs and between the top and bottom halves of the BVMs. These findings do not support the hypothesis that, after 1 day of cultivation of complex-shaped BVMs, no statistically significant differences would exist between the cell densities along the length of the BVMs, between the cell densities in the inner and outer halves of the BVMs, or between the cell densities in the top and bottom halves of the BVMs.

Although cell densities in some regions of the BVMs were statistically significantly different from each other, boxplots showed that the median cell density in Lshaped BVMs and the average cell density in U-shaped BVMs still fell between 250,000 cells $/ \mathrm{cm}^{2}$ and 350,000 cells $/ \mathrm{cm}^{2}$. Human native cell densities may vary, and animals have 
exhibited a range of endothelial cell densities within the confluent endothelium of a single blood vessel: canines have exhibited endothelial cell densities of $95,000 \mathrm{cells} / \mathrm{cm}^{2}$ in the ventral wall of the aortic arch orifice region but 155,000 cells $/ \mathrm{cm}^{2}$ in the lesser curvature of the aortic arch orifice region [75], and rabbits have exhibited endothelial cell densities of $381,700 \mathrm{cells} / \mathrm{cm}^{2}$ in their thoracic aorta but $401,600 \mathrm{cells} / \mathrm{cm}^{2}$ in their abdominal aorta [76]. Because a range of endothelial cell densities has been observed in the endothelium of native blood vessels, and because the cell densities in all L- and Ushaped BVMs were approximately the same order of magnitude as native endothelial cell densities, a complex-shaped BVM with the range of cell deposition that was identified in this chapter may still result in the development of a confluent cell lining in all regions of the complex-shaped BVM. Therefore, the statistically significantly different cell densities observed in this study may not necessarily lead to formation of unevenly confluent cell linings.

The statistical analysis of cell deposition that was presented in this chapter could be used in future studies. Future studies may result in the development of confluent cell linings in complex-shaped BVMs, and the statistical analysis from this chapter could be compared to the confluent cell linings to determine whether statistically significant differences in cell deposition affect the development of confluent cell linings. If statistically significant differences in cell deposition do affect development of confluent cell linings, cell deposition methods could be altered to improve development of confluent cell linings.

An issue occurred with the BBI imaging of cells in the outer half of one of the Ushaped BVMs: the edges of the cells were not clearly defined. A possible explanation for 
this is as follows. The surface of ePTFE consists of nodes and internodal fibrils (Figure 3.18), and the cells in Figure 3.8 D appeared to organize in stripes on the scaffold. The cells may have settled into the internodal regions, and BBI solution may have also settled into the internodal regions and been resistant to blotting with a Kimwipe. The BBI solution may not have been completely removed from the internodal regions, resulting in BBI images that showed blurry blue dots. Although some BBI images were blurry, most showed clearly identifiable blue dots, and the exclusion of the blurry BBI images from statistical analyses was not detrimental to obtaining useful information in this study.

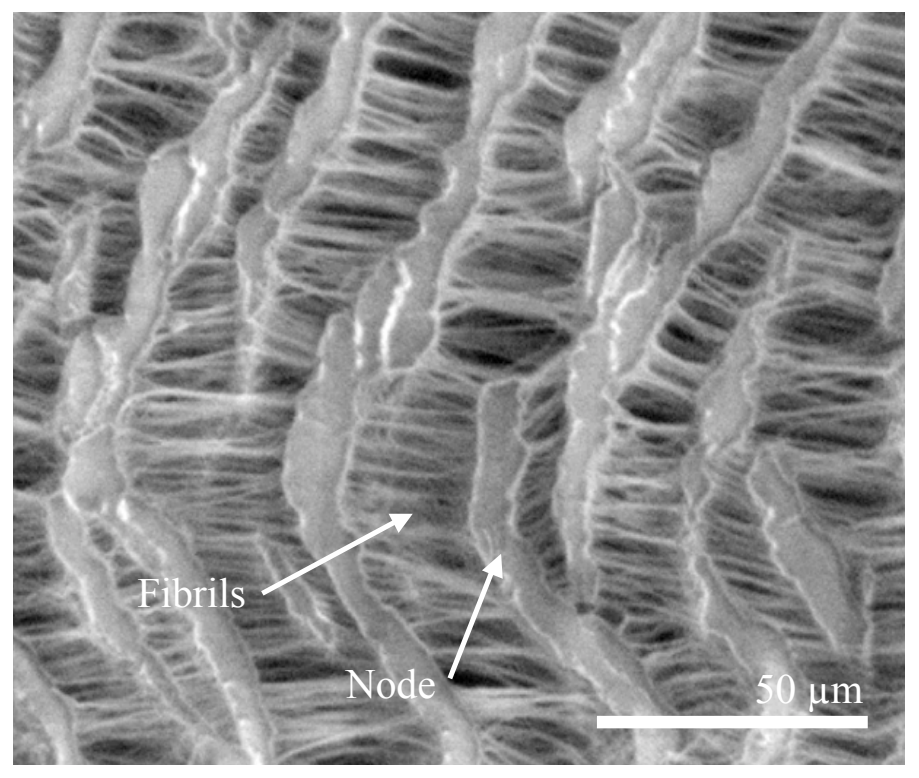

Figure 3.18. Luminal surface of eTPFE scaffold. Nodes are separated by fibrils. [77]

Although exclusion of the few blurry images was not detrimental to this study, there was a possible drawback to the exclusion. The statistical comparison between the cell densities in the inner and outer halves of U-shaped BVMs yielded a p-value of 0.057, conveying that no statistically significant difference existed between the cell densities in 
the inner and outer halves of U-shaped BVMs. But 0.057 was only slightly outside the range of $p$-values that would have conveyed that there was a statistically significant difference $(\mathrm{p}<0.05)$, and inclusion of the omitted cell density data may have reduced 0.057 to a p-value less than 0.05 . To confirm or refute these results, a larger batch of Ushaped BVMs could be tissue-engineered, and the statistical comparison could be performed again on the new U-shaped BVMs. But additional experiments that assess cell deposition should not be attempted before development of a confluent cell lining is attempted because development of a confluent cell lining on a complex-shaped scaffold is more useful for coronary stent testing than additional cell deposition information.

In conclusion, statistically significant differences existed between 5 regions along the length of L-shaped BVMs, between the inner and outer halves of L-shaped BVMs, and between 5 regions along the length of U-shaped BVMs. But cells deposited with average or median densities between 250,000 cells $/ \mathrm{cm}^{2}$ and 350,000 cells $/ \mathrm{cm}^{2}$ throughout all regions of L- and U-shaped BVMs, which may be sufficient for formation of a confluent cell lining throughout all regions of these complex-shaped BVMs. Therefore, development of a confluent cell lining in complex-shaped BVMs was attempted. 


\section{Effect of Flow Rate on Cell Confluence in U-Shaped BVMs}

\subsection{Introduction}

This chapter addresses the third specific aim of this thesis: determine whether HUVECs can reach confluence on a complex-shaped scaffold using a cultivation protocol similar to the straight-BVM cultivation protocol.

A potential first step toward determining whether HUVECs could reach confluence on a complex-shaped scaffold was to cultivate complex-shaped BVMs using the protocol that causes cells in the current straight BVM to reach confluence.

Development of a confluent cell lining in the current straight BVM involved cultivation of the straight BVM over a period of more than 1 day and also involved adjustment of the flow rate of media moving through the straight BVM lumen [59]. After pressure-sodding HUVECs onto straight ePTFE scaffolds, straight BVMs were first cultivated for 1 day at a flow rate of $1.6 \mathrm{~mL} / \mathrm{min}$, and this cultivation yielded cells that appeared balled-up, cell coverage that was sparse, and cells that did not reach confluence [59]. After this 1 day of cultivation, the flow rate was increased by $1.2 \mathrm{~mL} / \mathrm{min}$ every hour until the flow rate reached $12 \mathrm{~mL} / \mathrm{min}$ [59]. Straight BVMs were then harvested after 3, 5, and 7 days of cultivation [59]. After 3 days of cultivation, some cells were still balled-up, but cells started to reach confluence [59]. After 5 and 7 days of cultivation, cells were no longer balled-up, and cells had reached confluence [59].

Use of the straight BVM protocol to cause cells to reach confluence in complexshaped BVMs could present a challenge. In the previous chapter, cells were deposited

onto L- and U-shaped scaffolds at a density of 1.5 million cells $/ \mathrm{cm}^{2}$, but the average or 
median cell density in the complex-shaped BVMs after 1 day of cultivation under a flow rate of $1.2 \mathrm{~mL} / \mathrm{min}$ was $250,000-350,000$ cells/ $/ \mathrm{cm}^{2}$, conveying that approximately 1.2 million cells $/ \mathrm{cm}^{2}$ that were introduced into the complex-shaped scaffolds were not adhered to the scaffolds after the 1 day of cultivation under flow. One potential explanation for this is that flow pushes cells off complex-shaped scaffolds during cultivation. Also, previous work has shown that a 4-mm-ID straight ePTFE scaffold exhibits a wall shear stress of 0.225 dynes $/ \mathrm{cm}^{2}$ when fluid that has a viscosity similar to the viscosity of media flows luminally through the scaffold at a flow rate of $12 \mathrm{~mL} / \mathrm{min}$, whereas a 4-mm-ID U-shaped ePTFE scaffold exhibits a wall shear stress of 1.75 dynes $/ \mathrm{cm}^{2}$ when the same fluid flows luminally through the scaffold at the same flow rate - the wall shear stress of a U-shaped scaffold is more than 7 times the wall shear stress of a straight scaffold in this case [66]. Because flow may push cells off complexshaped scaffolds, and because U-shaped scaffolds experience higher wall shear stress than straight scaffolds under a flow rate of $12 \mathrm{~mL} / \mathrm{min}$, a potential outcome after cultivation of U-shaped and straight BVMs for 7 days at $12 \mathrm{~mL} / \mathrm{min}$ is that fewer cells remain adhered to U-shaped scaffolds than to straight scaffolds. If this potential outcome is true, the fewer cells of U-shaped BVMs may then not be able to reach confluence. To safeguard against this potential outcome, slower cultivation flow rates should be tested in U-shaped BVMs in addition to testing the $12 \mathrm{~mL} / \mathrm{min}$ that has been used to cultivate straight BVMs.

The slower cultivation flow rates that are tested should be the least likely flow rates that would push cells off scaffolds. A flow rate that would exert no force on cells is $0 \mathrm{~mL} / \mathrm{min}$. No flow may allow more cells to remain adhered to complex-shaped 
scaffolds, which may result in cells reaching confluence. Another flow rate that may be least likely to push cells off scaffolds is a gradually increasing flow rate. Gradually increasing flow rates are associated with better retention of cells on scaffolds than abruptly increasing flow rates $[78,79]$. The protocol for straight BVMs already includes a gradually increasing flow rate: after 1 day of cultivation, the flow rate is increased by $1.2 \mathrm{~mL} / \mathrm{min}$ every hour until the flow rate reaches $12 \mathrm{~mL} / \mathrm{min}$. But a flow rate that increases even more gradually could further reduce the chance that flow pushes cells off scaffolds. Therefore, complex-shaped BVMs were cultivated for 1 day using a cultivation protocol identical to the cultivation protocol described in the previous chapter, then the complex-shaped BVMs were further cultivated for up to 7 days at either $0 \mathrm{~mL} / \mathrm{min}$, a flow rate that gradually increased over multiple days, or almost identical flow rates used to cultivate straight BVMs. Cell confluence on the complex-shaped BVMs was assessed after 4 and 7 days of cultivation with the goal of showing development of a confluent cell lining over time.

To save time and resources and allow for faster discovery of successful laboratory techniques as in the previous two chapters, these experiments were only attempted in Ushaped BVMs. U-shaped BVMs were chosen because the FDA in various cases has recommended that coronary stents be tested in a worst-case clinically relevant radius of curvature. The hypothesis in this case was that after deposition of HUVECs onto Ushaped scaffolds and cultivation similar to the straight-BVM protocol, cells would reach confluence. 


\subsection{Materials and Methods}

6 U-shaped BVMs were cultivated in this study. For the first day of cultivation for all $6 \mathrm{BVMs}$, the cultivation protocol described in Section 3.2.6 was used. After the first day of cultivation, the BVMs were cultivated as follows: 2 BVMs at $0 \mathrm{~mL} / \mathrm{min}(1 \mathrm{BVM}$ for 4 days, 1 BVM for 7 days), 2 BVMs at an increasing flow rate (1 BVM for 4 days, 1 BVM for 7 days), and 2 BVMs at $12 \mathrm{~mL} / \mathrm{min}$ (1 BVM for 4 days, 1 BVM for 7 days). Each pair of BVMs was tissue-engineered during separate weeks so that each pair of BVMs could be attached to a separate pump with pump settings unique to that pair of BVMs. Cell culture, BVM cultivation, and BVM evaluation were all performed within a 3-week period, and a protocol that identifies when various laboratory techniques were used throughout the 3-week period is located in Appendix H. Each BVM was evaluated using histology, BBI imaging, and scanning electron microscopy (SEM).

\subsubsection{Cell Culture}

HUVECs (Lonza) were cultured and expanded in T75 and T225 BD cell culture flasks. Cells were cultured in HUVEC media, using the components and steps described in Appendix C. The cells were maintained at $37^{\circ} \mathrm{C}$ and $5 \% \mathrm{CO}_{2}$ in the same small incubator described in Section 3.2.1. During each passage from one flask to another flask, cells were monitored using the same inverted light microscope described in Section 3.2.1. When cells were pressure-sodded onto scaffolds, cells were no more than P8. Enough cells were cultured so that approximately 1.5 million cells $/ \mathrm{cm}^{2}$ were sodded onto each scaffold. 


\subsubsection{Sterilization and Media Preparation}

The 6 scaffolds were made of 4-mm-ID ePTFE, each piece cut to a length of 5.5 cm. Female luer thread barbed fittings were inserted into both ends of each scaffold, and both ends of each scaffold were sutured onto the fittings as described in Section 2.3.4. The scaffolds, multifunctional bioreactors and associated fittings, bioreactor lids, twoport lids, forceps, and a degassing flask were all packaged and sterilized as described in Section 3.2.2. Media was prepared as described in Section 3.2.2.

\subsubsection{Scaffold Preparation and Priming}

All scaffolds were denucleated and coated with protein as described in Section 3.2.3. Media was warmed in a water bath at $37^{\circ} \mathrm{C}$. The sterilized multifunctional bioreactors, lids, two-port lids for media reservoirs, and forceps were gathered. The scaffolds were inserted into multifunctional bioreactors in U-shapes using the same techniques as described in Section 3.2.4.

Scaffolds and reservoirs were primed with conditioning media as described in Section 3.2.4. Two-port lids were then connected to the bioreactors so that the inlets and outlets of the two-port lids were correctly connected to the inlets and outlets of the bioreactors. The complex-shaped BVM systems were attached to a peristaltic pump and primed for 10 minutes at $150 \mathrm{rpm}(12 \mathrm{~mL} / \mathrm{min})$. All inlets and outlets were closed, and the complex-shaped BVM systems were left in the large incubator. 


\subsubsection{Pressure-Sodding of HUVECs}

Cell culture was carried out so that 1 T225 flask of HUVECs was devoted to each scaffold. After the complex-shaped BVM systems were primed, the T225 flasks were removed from the small incubator, and the cells were inspected. Cells were removed from the bottom of the flasks using trypsin, and after all cells appeared to detach from the flask bottom, trypsin in the flasks was deactivated. The suspension of cells in each flask was transferred to its own 50-mL conical, and the conicals were centrifuged for 4 minutes. After centrifugation, the supernatant in the conicals was aspirated out, and the pellets of cells were resuspended as described in Section 3.2.5.

Complex-shaped BVM systems were then brought out of the large incubator and into the laminar flow hood as described in Section 3.2.5. Syringes were each filled with a volume of $4 \mathrm{~mL}$ of cell suspension, and all $4 \mathrm{~mL}$ of cell suspension were injected transmurally onto each scaffold. $5 \mathrm{~mL}$ of HUVEC media were then injected transmurally onto each scaffold. Then all inlets and outlets were closed to prevent media backflow from dislodging cells from scaffold pores, and the bioreactor outlets were reconnected to the reservoir tubing. The reservoirs containing conditioning media were replaced with reservoirs containing HUVEC media, the complex-shaped BVM systems were reattached to the peristaltic pump in the large incubator, and the pump was immediately turned on to a setting of $10 \mathrm{rpm}(0.8 \mathrm{~mL} / \mathrm{min})$ to administer transmural flow.

\subsubsection{Cultivation}

Transmural flow at $10 \mathrm{rpm}(0.8 \mathrm{~mL} / \mathrm{min})$ was maintained on all U-shaped BVMs for 1 hour. Then the luminal outlet on all bioreactors was opened, and luminal flow at 10 
$\operatorname{rpm}(0.8 \mathrm{~mL} / \mathrm{min})$ was maintained for another hour. Luminal flow was then increased to $15 \mathrm{rpm}(1.2 \mathrm{~mL} / \mathrm{min})$ and maintained overnight. After the first day of cultivation, each pair of BVMs was cultivated according to one of the following protocols. For the pair of BVMs allotted for cultivation at $0 \mathrm{~mL} / \mathrm{min}$, all inlets and outlets of the bioreactors were closed, and the pump was turned off and unplugged; 1 of the BVMs was harvested after 4 days, and 1 of the BVMs was harvested after 7 days. With the latter BVM, new media was placed in its reservoir after 3 days, and for 30 minutes every day until harvesting, the pump was turned on to $0.8 \mathrm{~mL} / \mathrm{min}(10 \mathrm{rpm})$ to circulate new media through the lumen of the latter BVM. For the pair of BVMs allotted for cultivation at a gradually increasing flow rate, the flow rate was increased by $1.2 \mathrm{~mL} / \mathrm{min}(15 \mathrm{rpm})$ each day until $9 \mathrm{~mL} / \mathrm{min}$ (100 rpm) was achieved after 4 days; 1 of the BVMs was harvested after 4 days, and 1 of the BVMs was harvested after 7 days with its media being changed after 3 days. For the pair of BVMs allotted for cultivation at $12 \mathrm{~mL} / \mathrm{min}$, the flow rate was increased by 1.2 $\mathrm{mL} / \mathrm{min}(15 \mathrm{rpm})$ every hour until $12 \mathrm{~mL} / \mathrm{min}(150 \mathrm{rpm})$ was achieved; 1 of the BVMs was harvested after 4 days, and 1 of the BVMs was harvested after 7 days with its media being changed after 3 days.

\subsubsection{Harvesting and Fixation}

At the end of each time point, BVMs were harvested as described in Section 3.2.7, including removal of the complex-shaped BVM systems from the large incubator, placement of the complex-shaped BVM systems on a nonsterile surface, removal of the BVMs from the bioreactors using a razor blade, and fixation of the BVMs in Histochoice. 


\subsubsection{Histologic Analysis, BBI Imaging, and SEM Imaging}

Imaging of all BVMs was conducted with the goal of assessing whether a confluent cell lining developed. All BVMs were first cut into the five segments shown in Figure 3.6 B. Segments $\mathrm{P}_{2}$ and $\mathrm{D}_{1}$ were histologically analyzed using the protocol located in Appendix L. Segments $\mathrm{P}_{1}, \mathrm{M}$, and $\mathrm{D}_{2}$ were each cut in half longitudinally so that each half had one of the blue lines that originally formed the double blue stripe on the PTFE scaffold. Segments $\mathrm{P}_{1}, \mathrm{M}$, and $\mathrm{D}_{2}$ were analyzed via $\mathrm{BBI}$ imaging using the protocol located in Appendix G, and segment $M$ was analyzed a second time via SEM imaging using the protocol located in Appendix K.

Segments allotted for histologic analysis were each embedded in paraffin wax at the Cal Poly Tissue Analysis Laboratory. The segments were oriented in the paraffin wax so that cross-sections could be sectioned. Cross-sections of the segments were then sectioned at 6-8 $\mu \mathrm{m}$ and stained with hematoxylin and eosin. White light microscopy was used to capture histology images of the entire circumference of the cross-sections. The detailed protocol used for these histology procedures is located in Appendix L.

Segments allotted for BBI imaging were stained with BBI solution. The BBI solution was prepared according to the protocol located in Appendix G. First, $1 \mathrm{~mL}$ of BBI solution was placed in enough foil-wrapped $15-\mathrm{mL}$ conicals so that each segment was allotted a conical. The conicals were labeled with information that identified the specific segment that was stored in that conical (e.g. "U-Shaped BVM, $0 \mathrm{~mL} / \mathrm{min}$, 4-day, $\mathrm{P}_{1}$, inner"). The segments were left in the BBI solution overnight and then imaged according to the protocol located in Appendix G. 4 images were taken of each scaffold segment as described in Figure 3.6 C. 
Segments allotted for SEM imaging were first dehydrated in various dilutions of ethanol as described in Appendix K. The segments were then dried overnight in a dessicator. Segments were secured onto the specimen stand of a scanning electron microscope, and 3 SEM images were captured evenly along the length of each segment.

\subsection{Results}

Multiple histologic cross-sections were obtained from each BVM, and a representative histologic cross-section from each BVM was selected for inclusion in this results section. A representative BBI image and a representative SEM image from each BVM were also selected for inclusion in this results section. Histologic cross-sections, BBI images, and SEM images for all BVMs showed cells that had not reached confluence. Figures 4.1-4.18 show histologic images, BBI images, and SEM images for all 6 BVMs, and a summary of the results is located in Table 4.1.

Table 4.1. Effect of Flow Rate on Confluence of Cell Lining in U-shaped BVMs

\begin{tabular}{|c|c|c|c|}
\hline Flow Rate & $\begin{array}{c}\text { 4 Days of } \\
\text { Cultivation }\end{array}$ & $\begin{array}{c}\mathbf{7} \text { Days of } \\
\text { Cultivation }\end{array}$ & $\begin{array}{c}\text { Cells Reached } \\
\text { Confluence? }\end{array}$ \\
\hline $\mathbf{0 ~} \mathbf{~} \mathbf{L} / \mathbf{m i n}$ & $\begin{array}{c}\text { Sparse cell coverage } \\
\text { (Figures 4.1-4.3) }\end{array}$ & $\begin{array}{c}\text { Sparse cell coverage } \\
\text { (Figures 4.4-4.6) }\end{array}$ & No \\
\hline $\begin{array}{c}\text { Increasing over } \\
\text { multiple days }\end{array}$ & $\begin{array}{c}\text { Sparse cell coverage } \\
\text { (Figures 4.7-4.9) }\end{array}$ & $\begin{array}{c}\text { Sparse cell coverage } \\
\text { (Figures 4.10-4.12) }\end{array}$ & No \\
\hline $\mathbf{1 2} \mathbf{~} \mathbf{m L} / \mathbf{m i n}$ & $\begin{array}{c}\text { Sparse cell coverage } \\
\text { (Figures 4.13-4.15) }\end{array}$ & $\begin{array}{c}\text { Sparse cell coverage } \\
\text { (Figures 4.16-4.18) }\end{array}$ & No \\
\hline
\end{tabular}




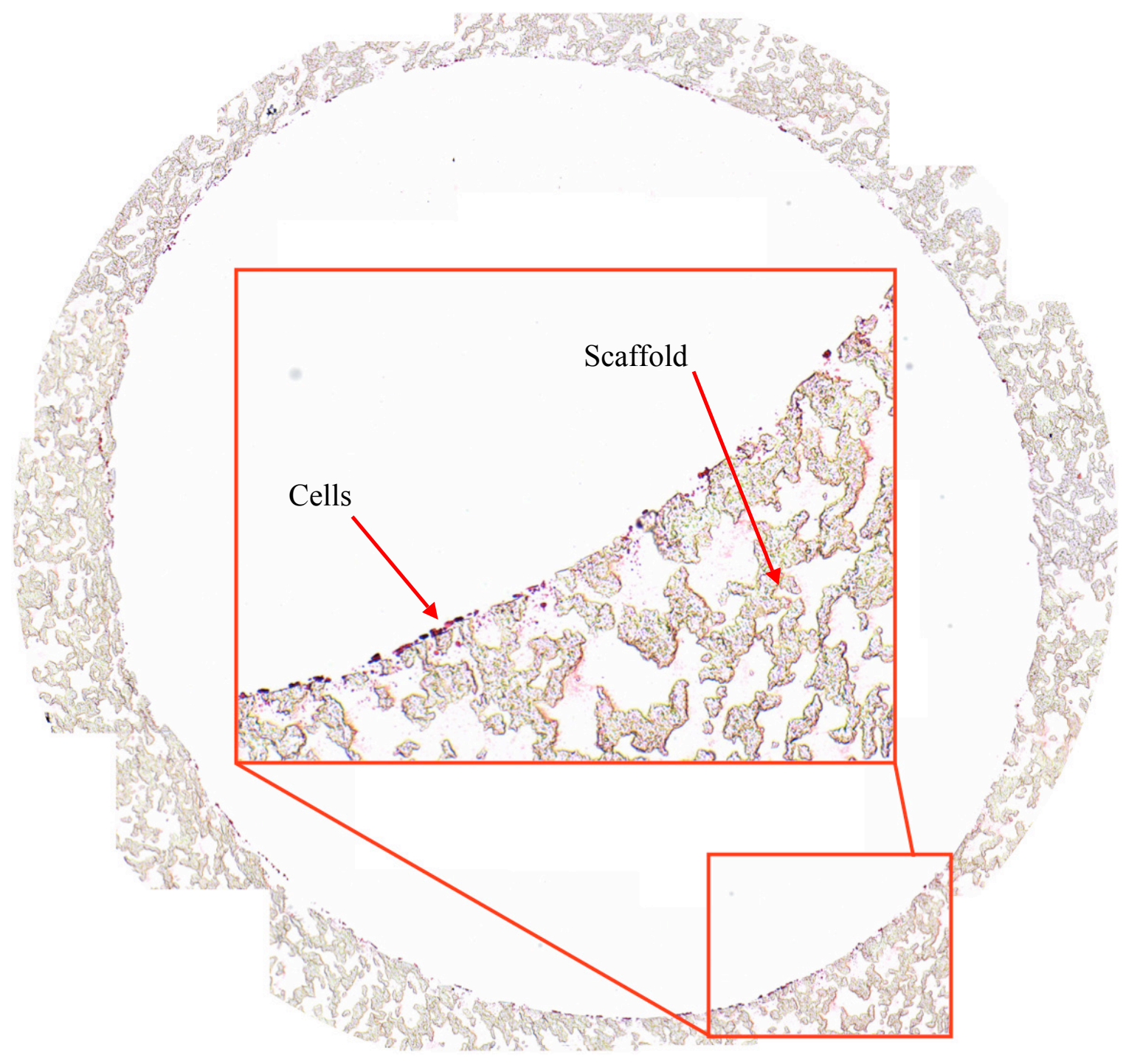

Figure 4.1. Histologic cross-section of a U-shaped BVM cultivated at $0 \mathrm{~mL} / \mathrm{min}$ for 4 days. The thick beige porous circle is the ePTFE scaffold, and the small dark purple spots on the inner surface of the scaffold are cells. Around the entire circumference of the cross-section, cells appear balled-up, cell coverage is sparse, and cells have not reached confluence. 


\section{$100 \mu \mathrm{m}$}

Figure 4.2. BBI image of a U-shaped BVM cultivated at $0 \mathrm{~mL} / \mathrm{min}$ for 4 days. Sparse blue dots confirm the sparse cell coverage shown in Figure 4.1.

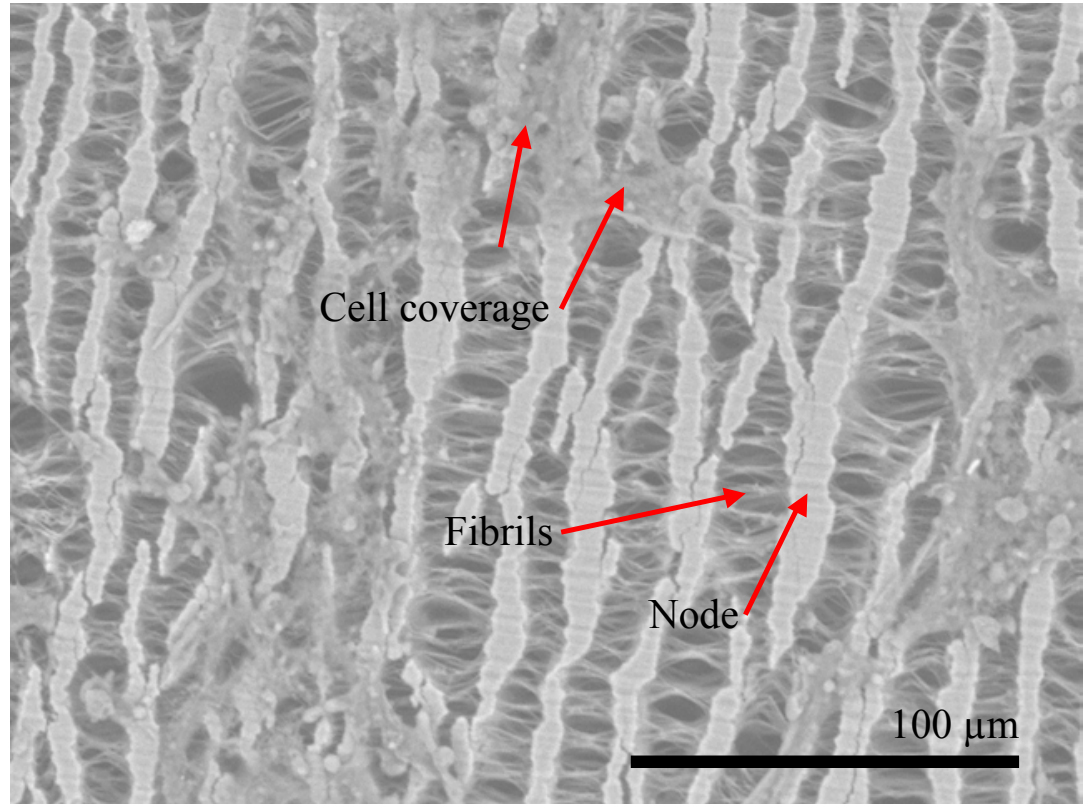

Figure 4.3. SEM image of a U-shaped BVM cultivated at $0 \mathrm{~mL} / \mathrm{min}$ for 4 days. Thick vertical lines are a part of the scaffold known as nodes, and the nodes are separated by scaffold fibrils. The few cells confirm the sparse cell coverage shown in Figure 4.1. 


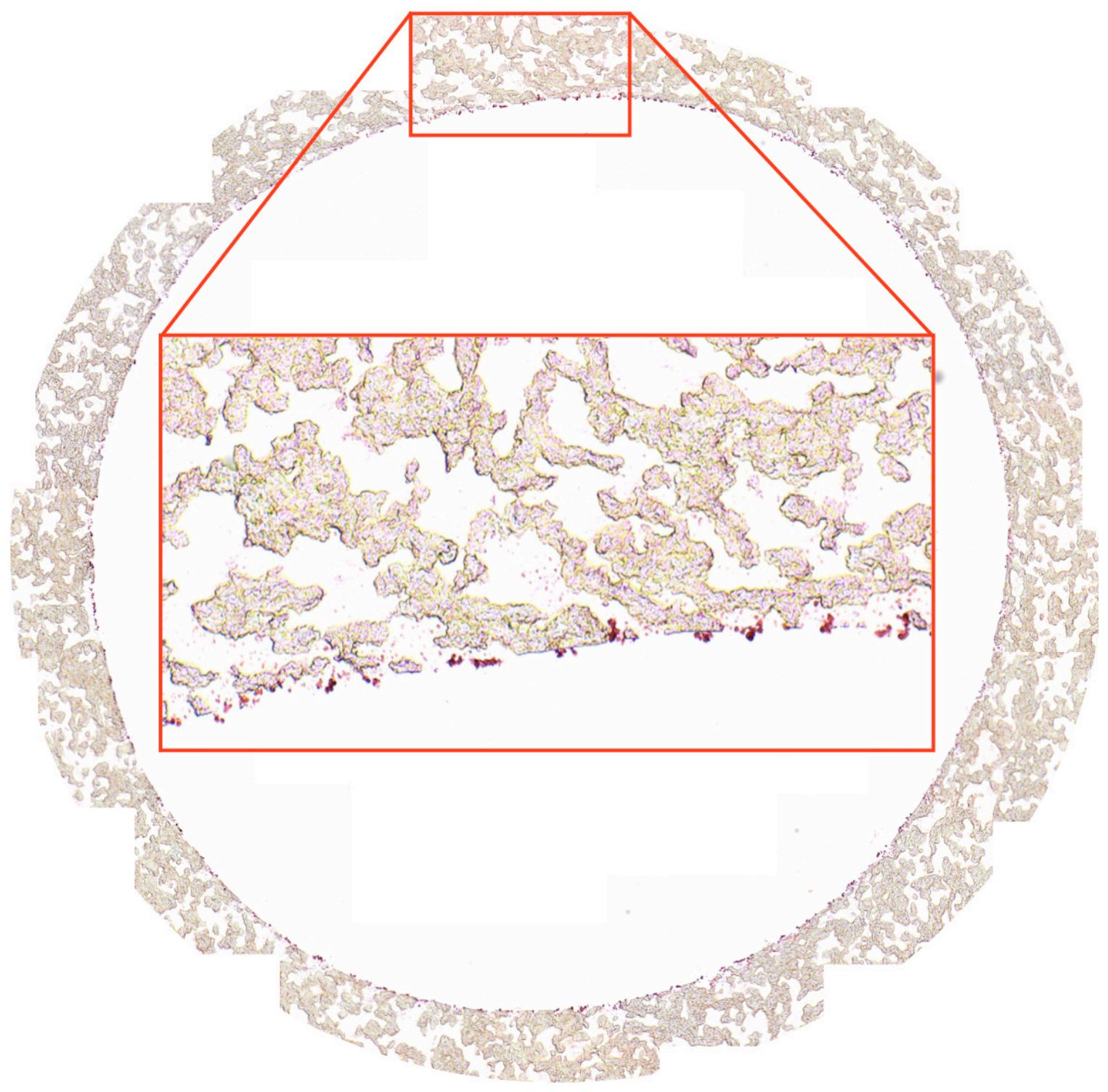

Figure 4.4. Histologic cross-section of a U-shaped BVM cultivated at $0 \mathrm{~mL} / \mathrm{min}$ for 7 days. Around the entire circumference of the cross-section, cells appear balled-up, cell coverage is sparse, and cells have not reached confluence. 
Figure 4.5. BBI image of a U-shaped BVM cultivated at $0 \mathrm{~mL} / \mathrm{min}$ for 7 days. Sparse blue dots confirm the sparse cell coverage shown in Figure 4.4.

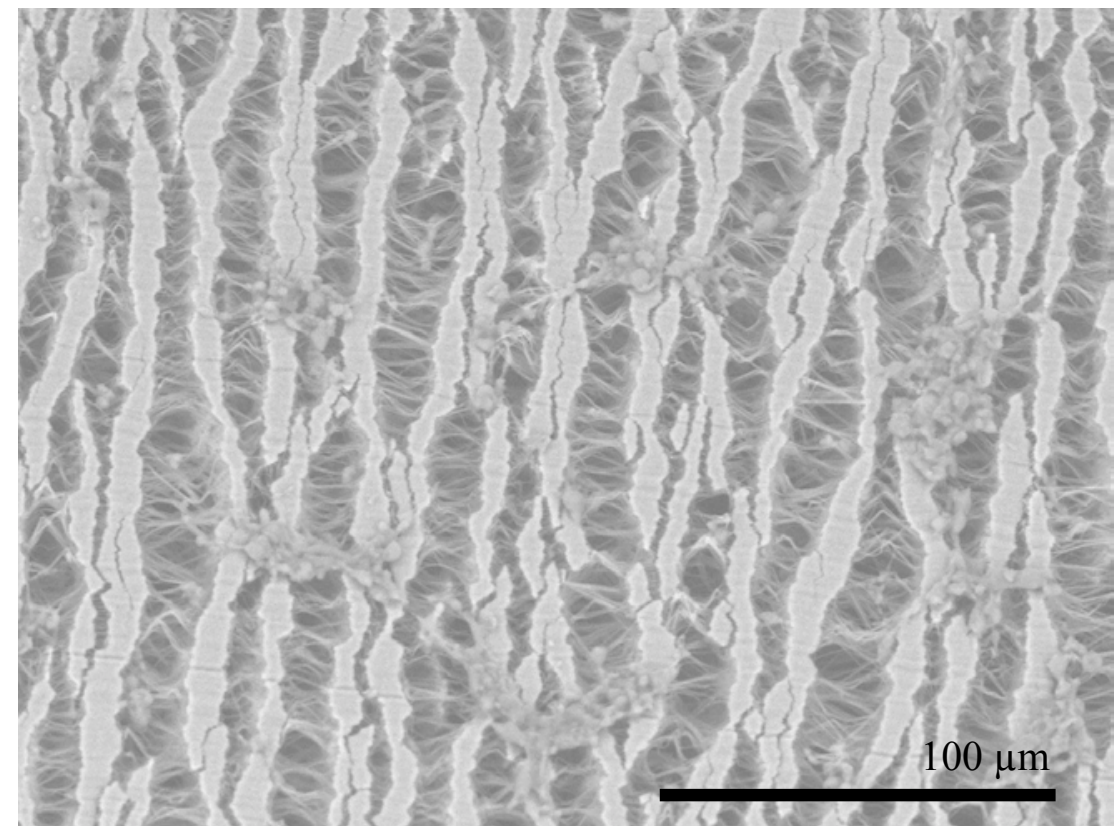

Figure 4.6. SEM image of a U-shaped BVM cultivated at $0 \mathrm{~mL} / \mathrm{min}$ for 7 days. The few cells confirm the sparse cell coverage shown in Figure 4.4. 


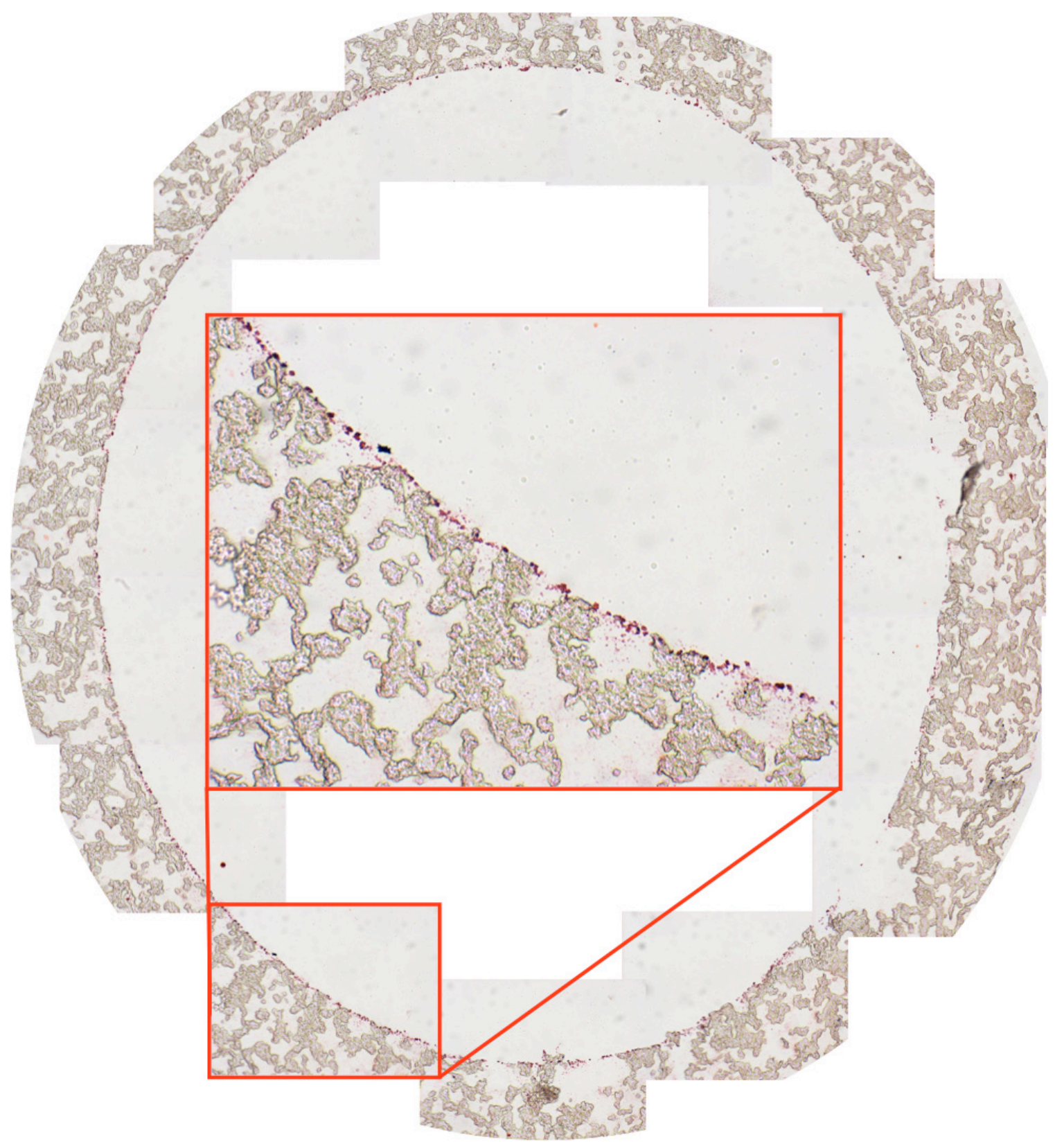

Figure 4.7. Histologic cross-section of a U-shaped BVM cultivated at an increasing flow rate for 4 days. Around the entire circumference of the cross-section, cells appear balledup, cell coverage is sparse, and cells have not reached confluence. 
Figure 4.8. BBI image of a U-shaped BVM cultivated at an increasing flow rate for 4 days. Sparse blue dots confirm the sparse cell coverage shown in Figure 4.7.

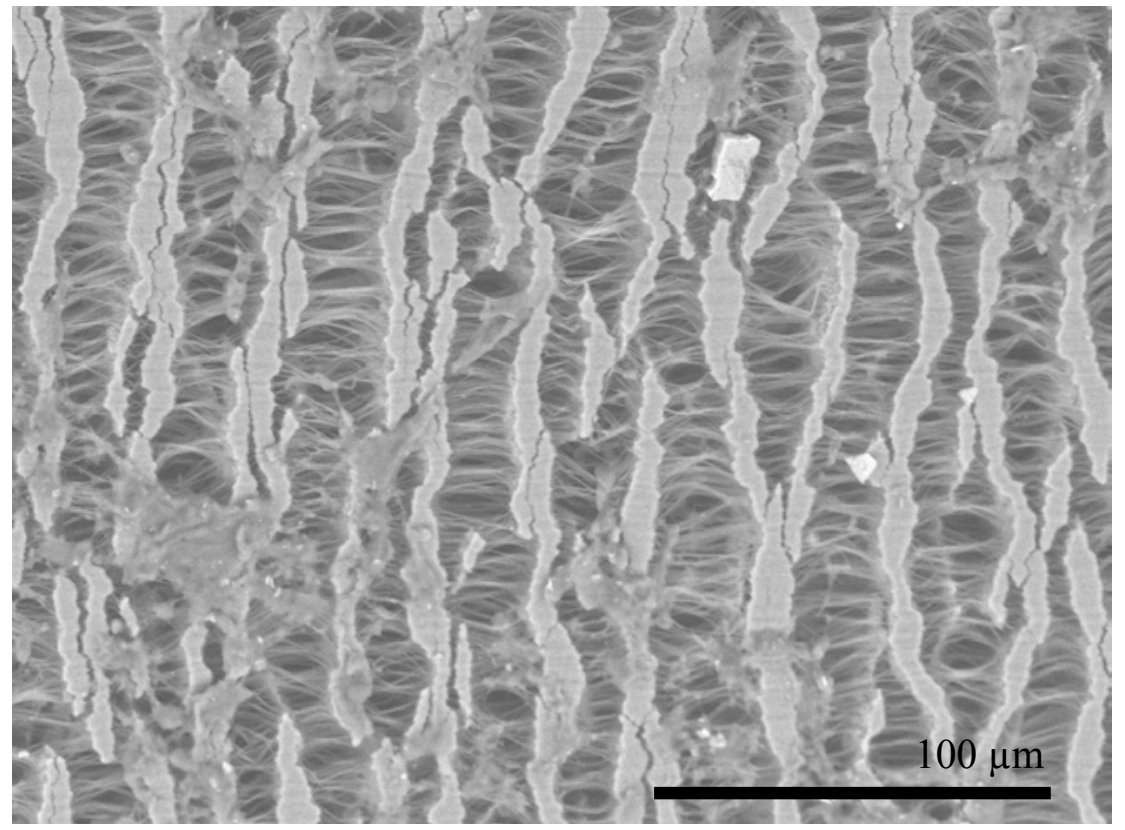

Figure 4.9. SEM image of a U-shaped BVM cultivated at an increasing flow rate for 4 days. The few cells confirm the sparse cell coverage shown in Figure 4.7. 


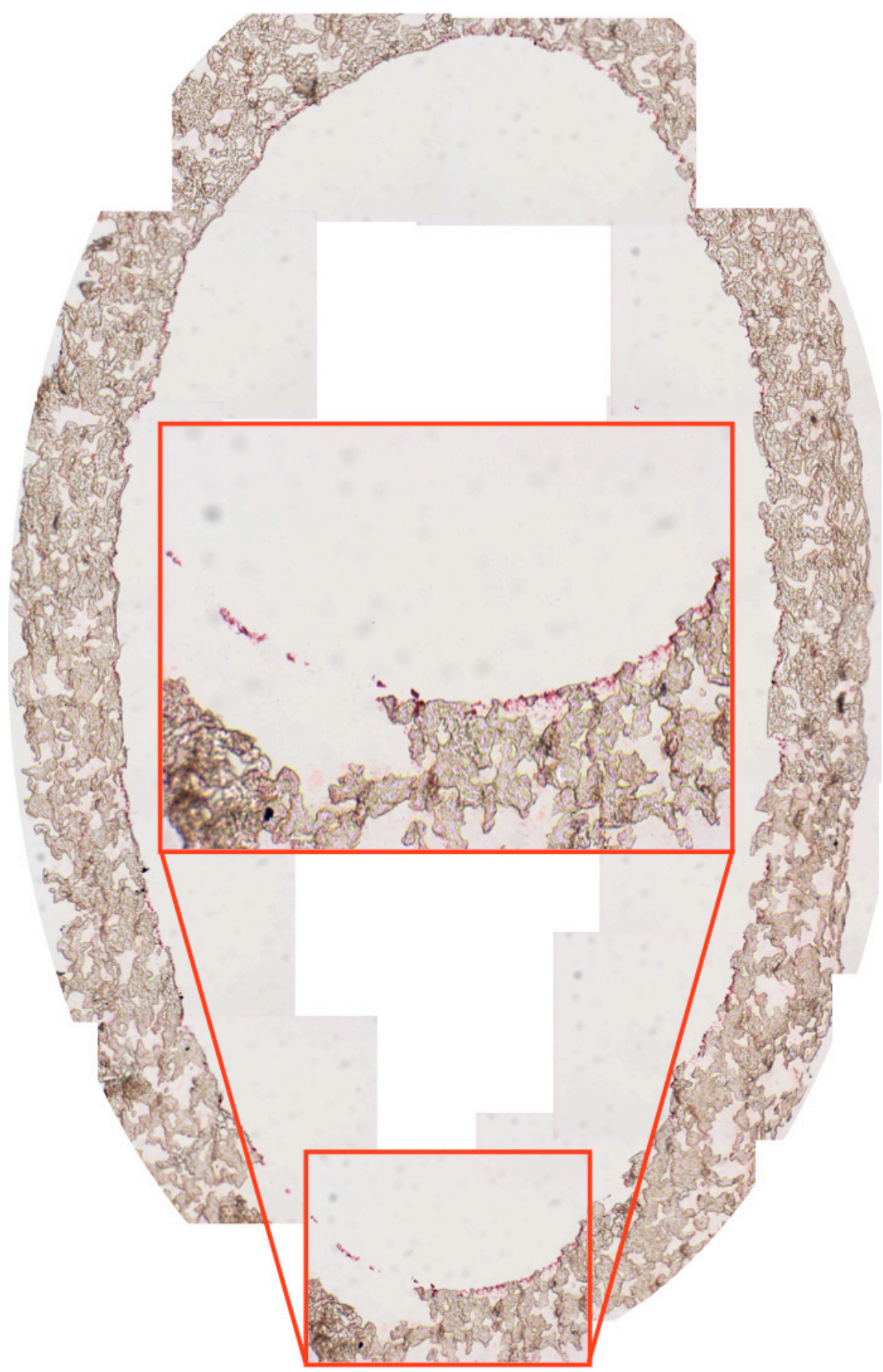

Figure 4.10. Histologic cross-section of a U-shaped BVM cultivated at an increasing flow rate for 7 days. Air was in the lumen of this BVM just before harvest. Around the entire circumference of this cross-section, cells appear fragmented, cell coverage is sparse, and cells have not reached confluence. 
Figure 4.11. BBI image of a U-shaped BVM cultivated at an increasing flow rate for 7 days. Sparse blue dots confirm the sparse cell coverage shown in Figure 4.10.

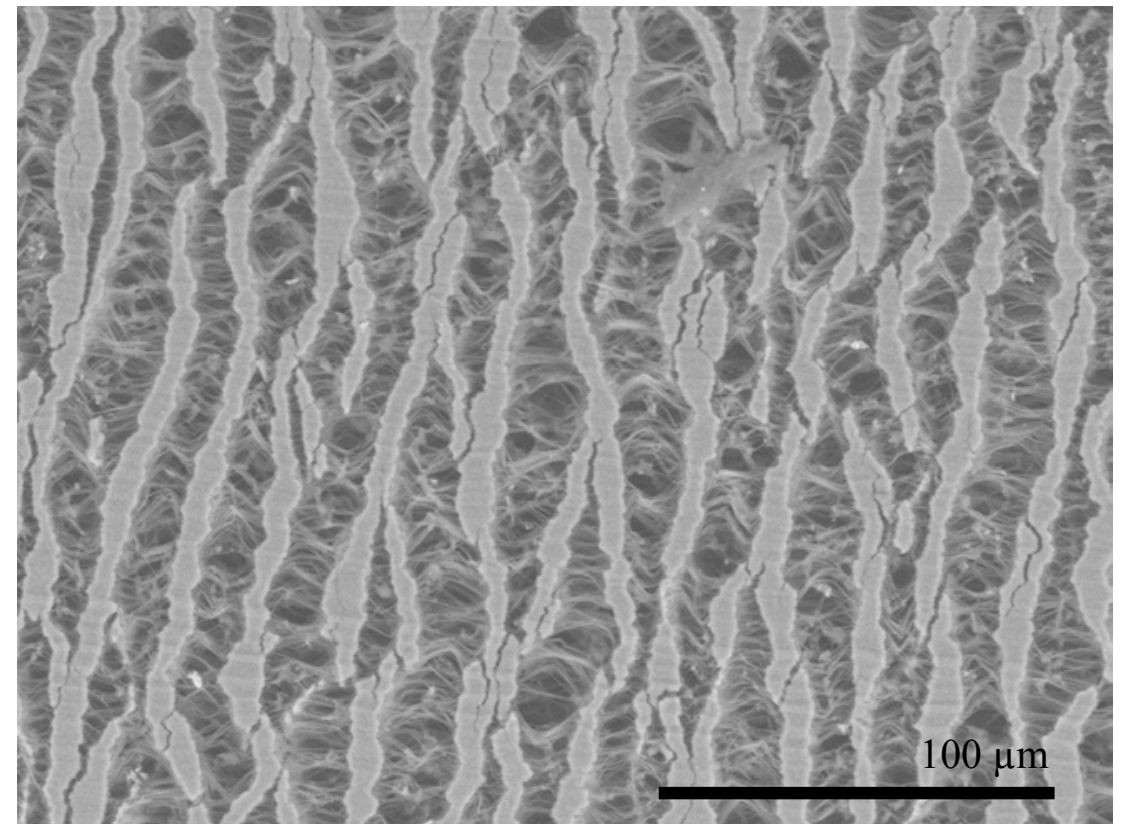

Figure 4.12. SEM image of a U-shaped BVM cultivated at an increasing flow rate for 7 days. The few cells confirm the sparse cell coverage shown in Figure 4.10. 


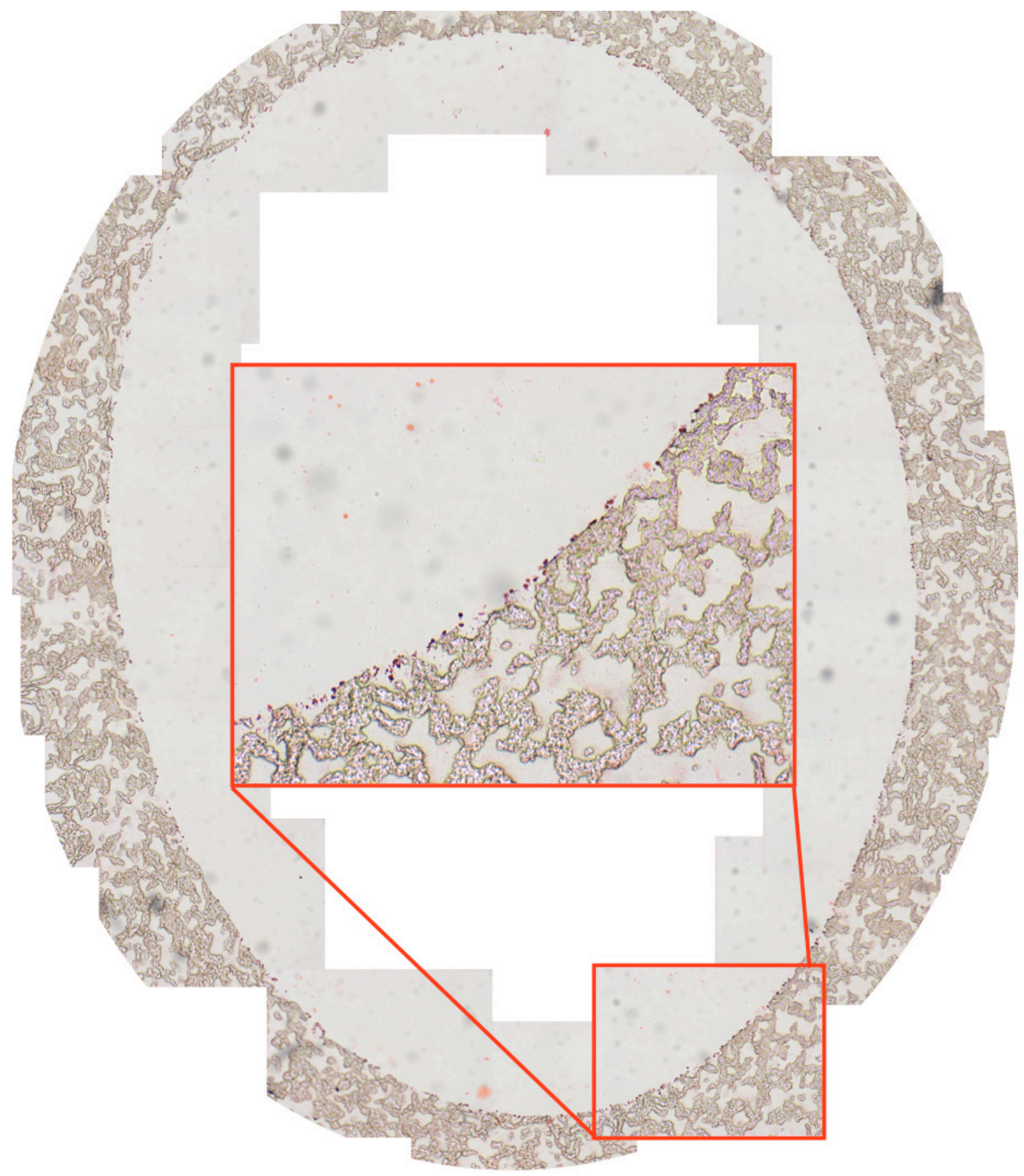

Figure 4.13. Histologic cross-section of a U-shaped BVM cultivated at a flow rate of 12 $\mathrm{mL} / \mathrm{min}$ for 4 days. Around the entire circumference of the cross-section, cells appear balled-up, cell coverage is sparse, and cells have not reached confluence. 


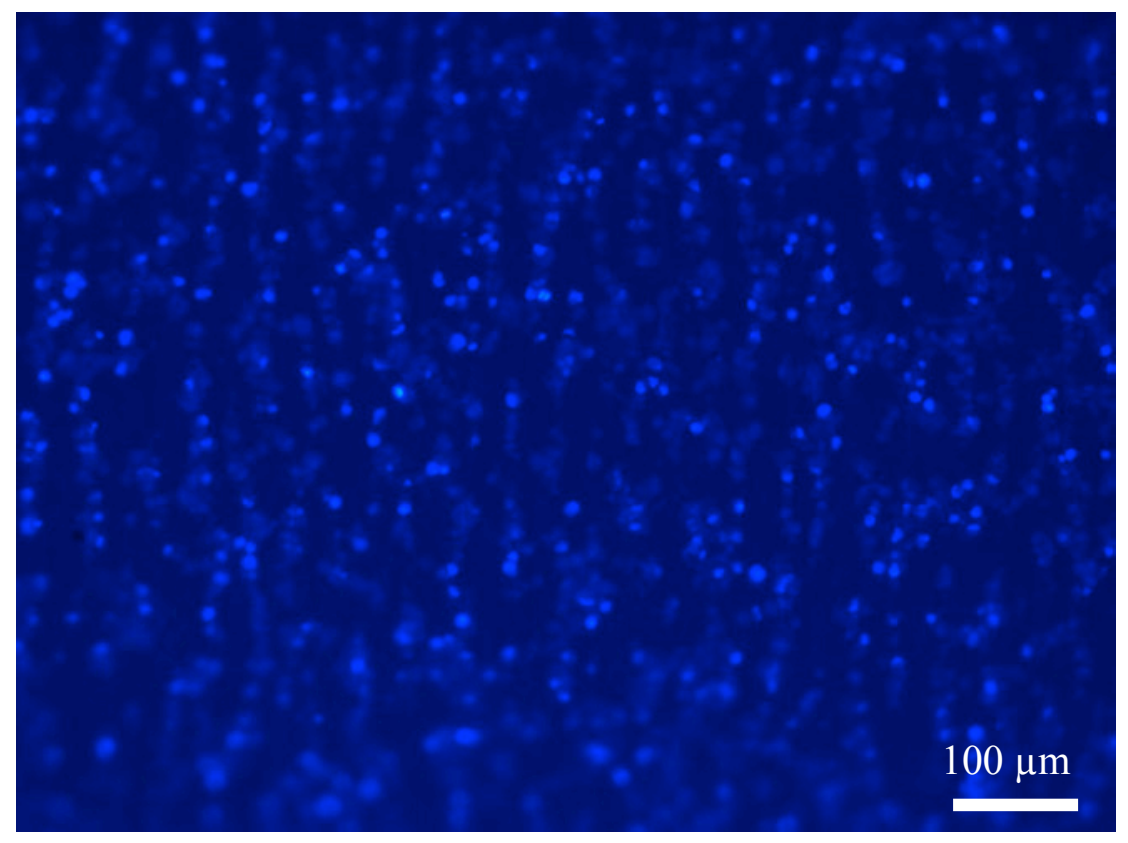

Figure 4.14. BBI image of a U-shaped BVM cultivated at a flow rate of $12 \mathrm{~mL} / \mathrm{min}$ for 4 days. Sparse blue dots confirm the sparse cell coverage shown in Figure 4.13.

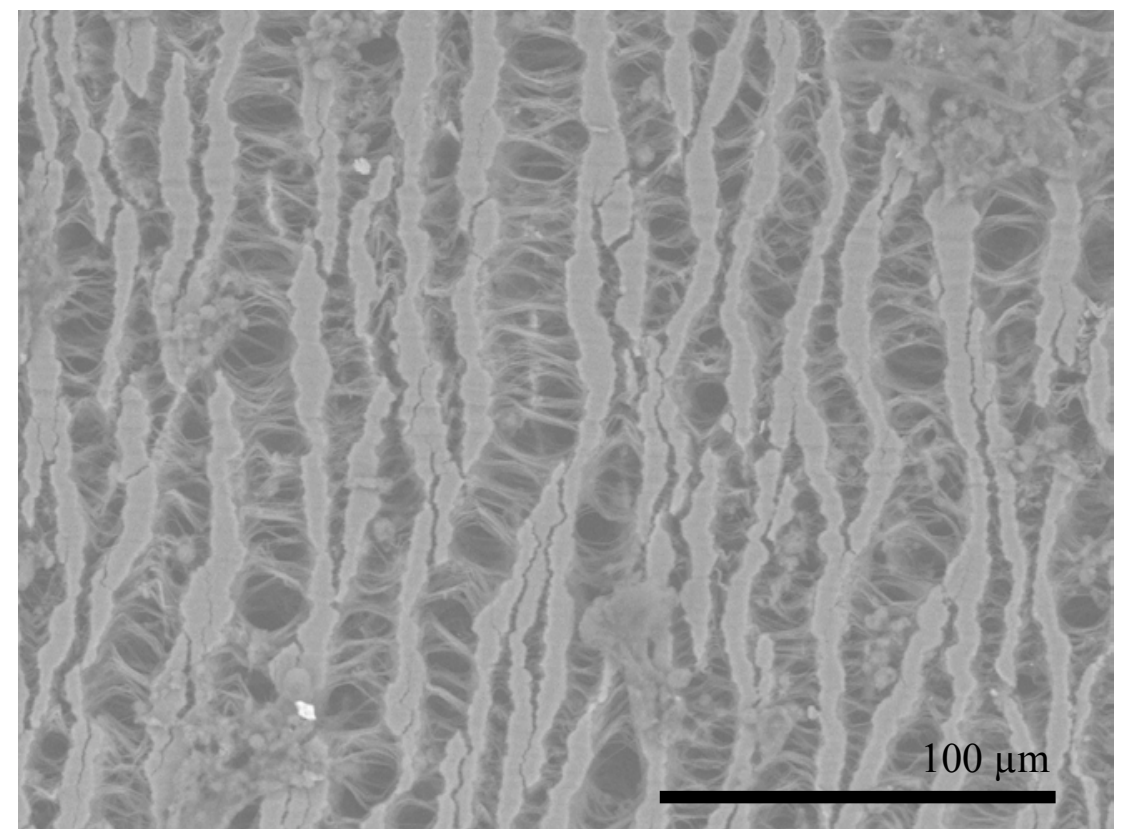

Figure 4.15. SEM image of a U-shaped BVM cultivated at a flow rate of $12 \mathrm{~mL} / \mathrm{min}$ for 4 days. The few cells confirm the sparse cell coverage shown in Figure 4.13. 


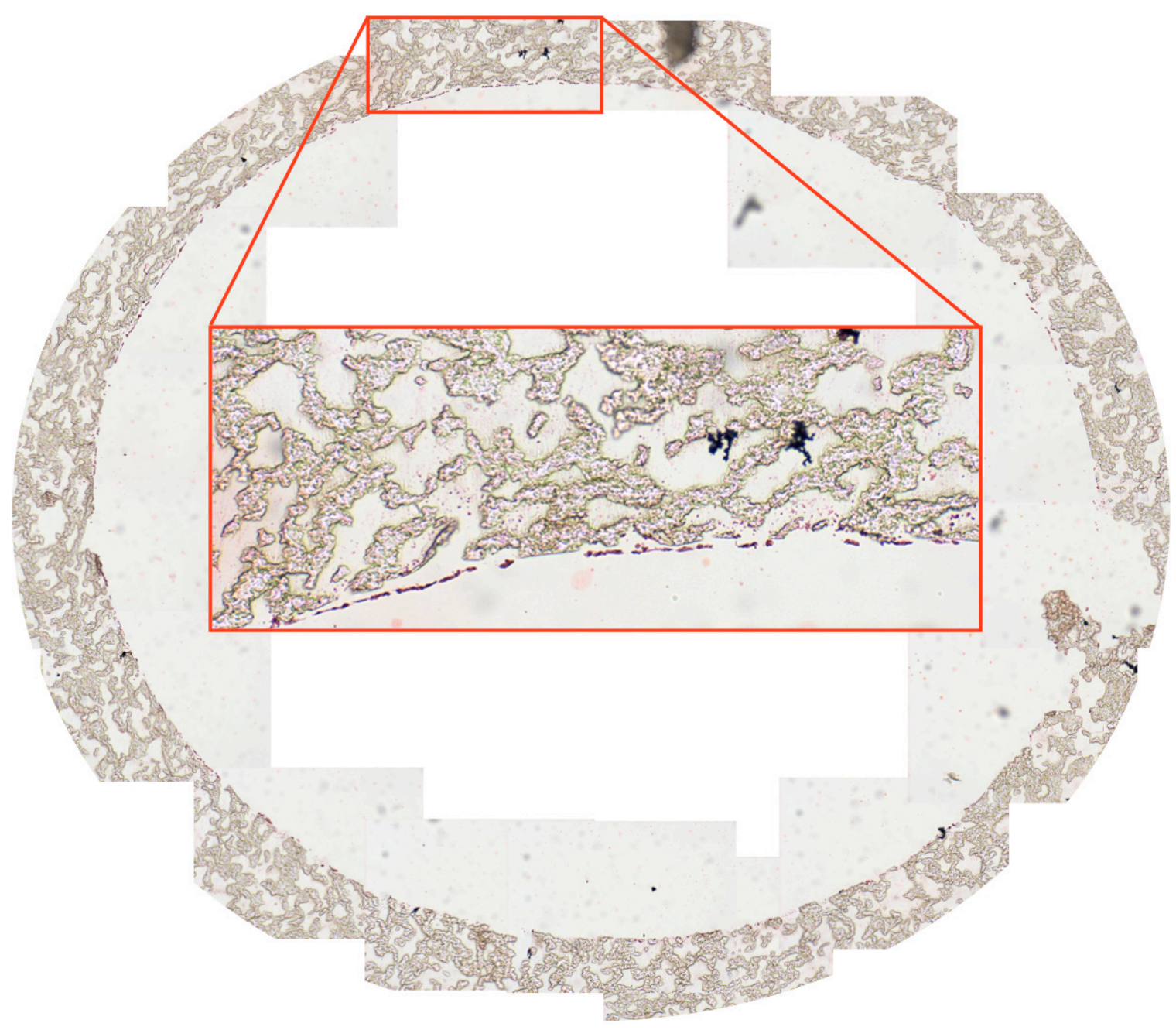

Figure 4.16. Histologic cross-section of a U-shaped BVM cultivated at a flow rate of 12 $\mathrm{mL} / \mathrm{min}$ for 7 days. Around the entire circumference of the cross-section, cell coverage is still sparse, and cells have not reached confluence. But cells have flattened out. 


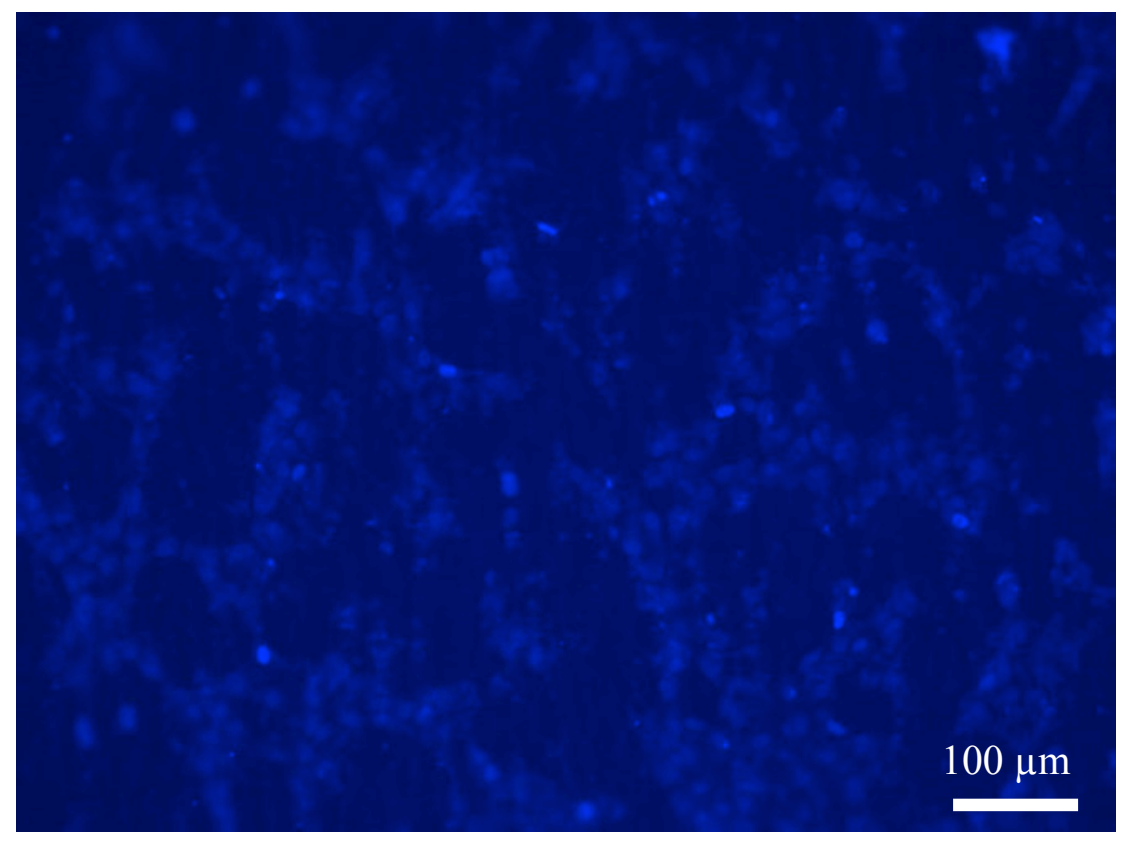

Figure 4.17. BBI image of a U-shaped BVM cultivated at a flow rate of $12 \mathrm{~mL} / \mathrm{min}$ for 7 days. Sparse blue dots confirm the sparse cell coverage shown in Figure 4.16.

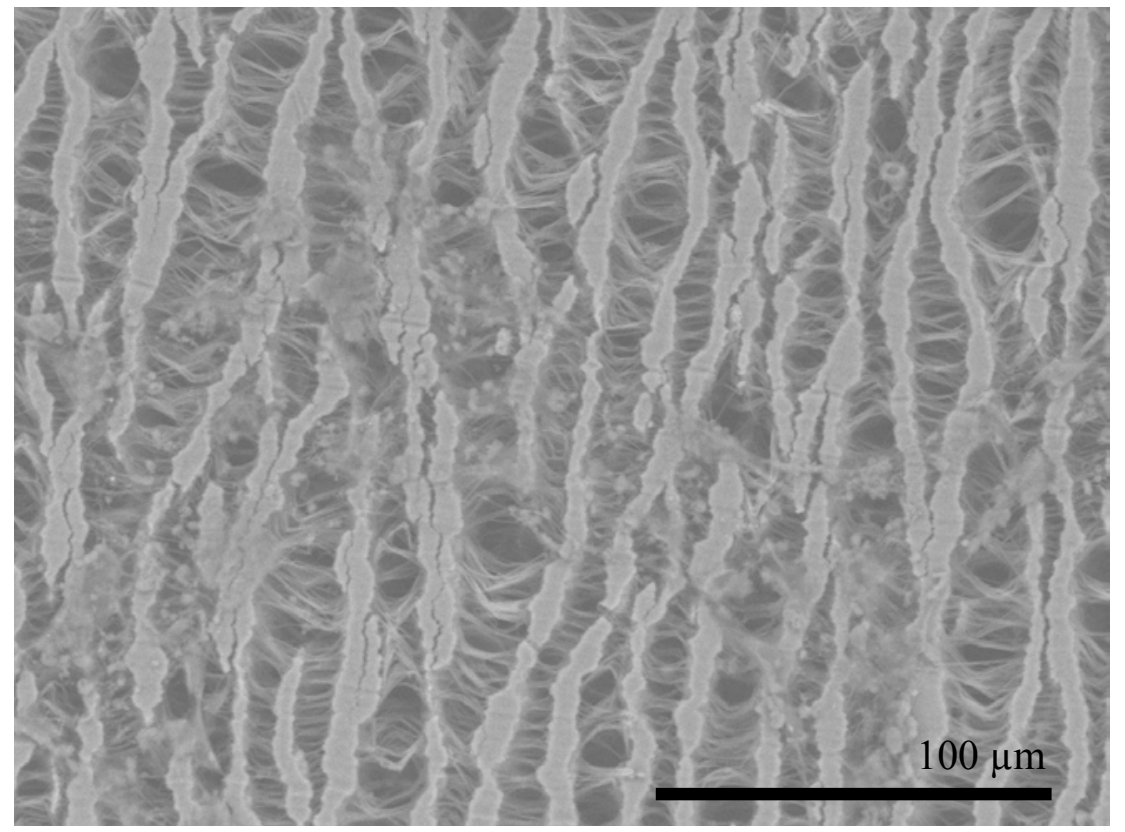

Figure 4.18. SEM image of a U-shaped BVM cultivated at a flow rate of $12 \mathrm{~mL} / \mathrm{min}$ for 7 days. The few cells confirm the sparse cell coverage shown in Figure 4.16. 
Throughout this study, two issues occurred with the complex-shaped BVM systems. First, an O-ring inside one of the multifunctional bioreactors broke apart during the first day of cultivation. Second, the two-port lid associated with the BVM that was exposed to increasing flow and that was harvested after 7 days leaked during priming of the two-port lid. Two-port lids have two fittings that are connected to tubing, and media leaked from the fittings of the two-port lid during priming of the reservoir.

\subsection{Discussion and Conclusions}

Whether HUVECs could reach confluence on a complex-shaped scaffold using a cultivation protocol that was similar to the straight-BVM cultivation protocol was determined in this chapter. A confluent cell lining has been achieved in a straight BVM by pressure-sodding HUVECs onto a straight ePTFE scaffold, then cultivating the straight BVM for 1 day at a flow rate of $1.6 \mathrm{~mL} / \mathrm{min}$, then increasing the flow rate by 1.2 $\mathrm{mL} / \mathrm{min}$ every hour until the flow rate reaches $12 \mathrm{~mL} / \mathrm{min}$, and then cultivating the straight BVM at $12 \mathrm{~mL} / \mathrm{min}$ for 6 more days [59]. An identical cultivation protocol was used to cultivate U-shaped BVMs, but cells did not reach confluence. Because flow in Ushaped scaffolds may push more cells off the scaffolds than flow in straight scaffolds, possibly resulting in subconfluent cell linings in U-shaped scaffolds, slower flow rates were also used to cultivate U-shaped BVMs. But after cultivating U-shaped BVMs using $0 \mathrm{~mL} / \mathrm{min}$ and a gradually increasing flow rate that did not exceed $12 \mathrm{~mL} / \mathrm{min}$, cells still did not reach confluence. Therefore, these findings did not support the hypothesis that after deposition of HUVECs onto U-shaped scaffolds and cultivation similar to the straight-BVM protocol, cells would reach confluence. 
These findings do reveal information about the effect of flow rate on cells in Ushaped BVMs. As seen in the previous chapter, after 1 day of cultivation at a flow rate of $1.2 \mathrm{~mL} / \mathrm{min}$, U-shaped BVMs exhibited dense cell coverage (Figure $4.19 \mathrm{~A}$ ). Then after 3 more days of cultivation at a flow rate of $12 \mathrm{~mL} / \mathrm{min}$, U-shaped BVMs exhibited noticeably sparser cell coverage (Figure 4.19 B). Therefore, U-shaped BVMs may exhibit a loss of cells when cultivated at $12 \mathrm{~mL} / \mathrm{min}$ after the first day of cultivation. But even when the 3 additional days of cultivation occurred under no flow at all, U-shaped BVMs still exhibited a noticeable loss of cells (Figure 4.19 C). Therefore, flow may not cause the noticeable loss of cells; instead, the scaffold may cause the noticeable loss of cells. But, although the loss of cells with flow (Figure 4.19 B) does not appear significantly larger than the loss of cells without flow (Figure $4.19 \mathrm{C}$ ), the loss of cells could be statistically significantly larger with flow than without flow. If the loss of cells is statistically significantly larger with flow than without flow, flow may still be pushing cells off the scaffold even though it may not be noticeable to the naked eye in Figures 4.19 B and C. 

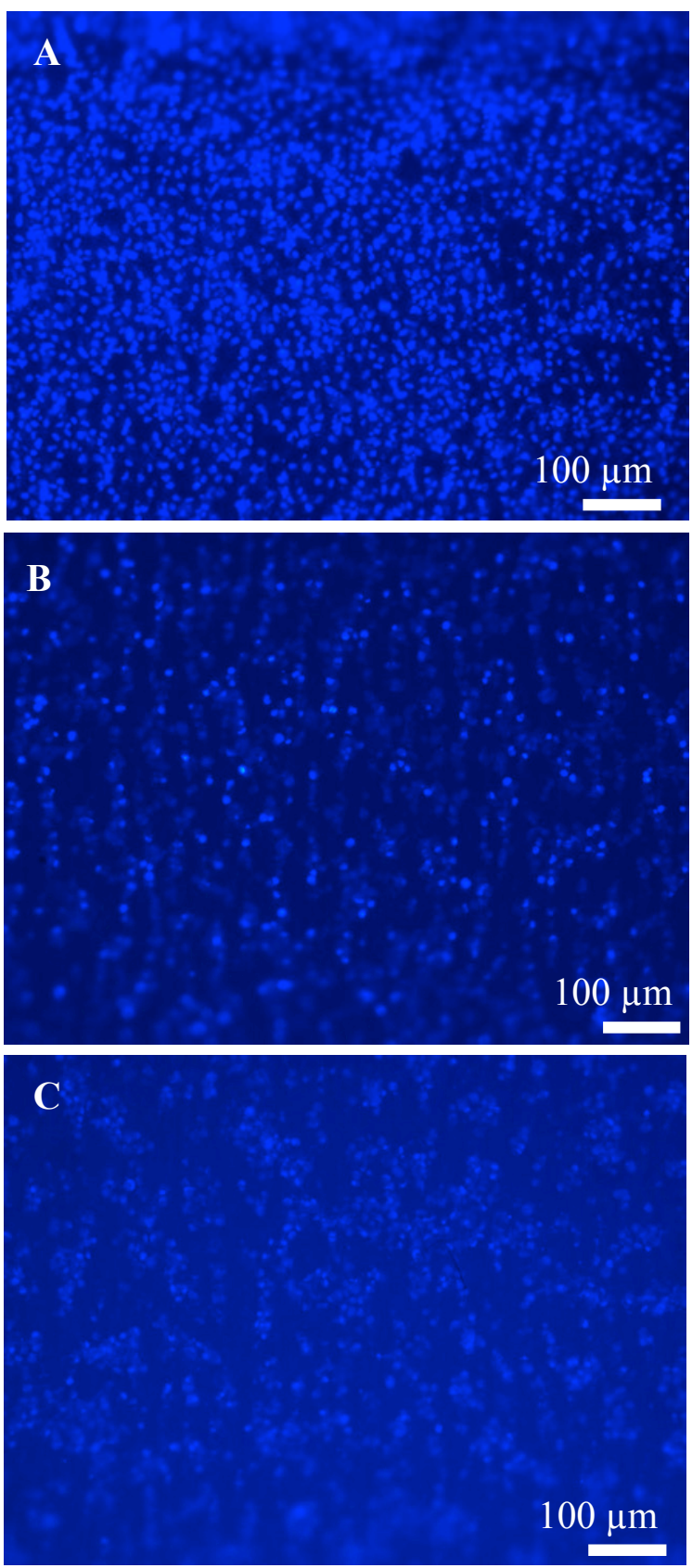

Figure 4.19. Cell loss after first day of cultivation of U-shaped BVMs. With 1 day of cultivation, U-shaped BVMs exhibit dense cell coverage (A). After an additional 3 days of cultivation at a flow rate of $12 \mathrm{~mL} / \mathrm{min}$, U-shaped BVMs exhibited a noticeable loss of cells (B). But even after the additional 3 days of cultivation under no flow at all, Ushaped BVMs still exhibited a noticeable loss of cells (C). 
If flow pushes cells off the scaffold, flow may also be responsible for cells not reaching confluence in U-shaped BVMs after 7 days of cultivation. When straight BVMs are cultivated for 1 day at a flow rate of $1.6 \mathrm{~mL} / \mathrm{min}$ and then for 2 additional days at a flow rate of $12 \mathrm{~mL} / \mathrm{min}$, straight BVMs exhibit sparse cell coverage [59], and sparse cell coverage also occurs around the same time point in U-shaped BVMs that have been cultivated at almost identical flow rates. But straight BVMs then go on to exhibit a confluent cell lining after 7 days of cultivation, while U-shaped BVMs go on to still exhibit sparse cell coverage after 7 days of cultivation. A potential explanation for this is described as follows. The unique flow in a U-shaped scaffold that results in higher wall shear stress may push more cells off the scaffold than the flow in a straight scaffold that results in lower wall shear stress, resulting in more spaces of bare scaffold between cells on the U-shaped scaffold. Cells on a straight scaffold may then only have to spread out to contact neighboring cells, whereas cells on a U-shaped scaffold may have to spread out but also migrate to contact neighboring cells. During 7 days of cultivation, cells on the Ushaped scaffold may have been continuously pushed off the scaffold before contacting any neighboring cells and therefore may never have been able to reach confluence, whereas cells on the straight scaffold may have been pushed off the scaffold less often, were therefore able to contact neighboring cells more easily, and were therefore able to reach confluence. Again, this explanation is possible only if the loss of cells between 1 and 4 days of cultivation is statistically significantly larger with flow than without flow.

A limitation of the experimental design of this study was that no 1-day U-shaped BVMs were developed as controls to monitor cell deposition on the scaffolds. Although the multiple 1-day U-shaped BVMs in the previous chapter all exhibited average or 
median densities between 250,000 cells $/ \mathrm{cm}^{2}$ and 350,000 cells $/ \mathrm{cm}^{2}$, it is still possible that the BVMs described in the current chapter exhibited significantly less cell deposition, resulting in subconfluent cell linings. For example, any missteps during pressure-sodding may have resulted in significantly less cell deposition. Therefore, it is possible that significantly less cell deposition — not high wall shear stress nor the scaffold — caused the subconfluent cell linings seen in this chapter. Future studies that aim to assess cell lining development over time should therefore always include 1-day control BVMs so that cell deposition is monitored.

Because the protocols for tissue-engineering a U-shaped BVM that were proposed in this chapter did not cause cells to reach confluence, one of the proposed protocols must be selected as the best proposed protocol and then altered in a way that will likely cause cells to reach confluence. The U-shaped BVMs that were cultivated with the flow rate of $12 \mathrm{~mL} / \mathrm{min}$ did exhibit flattened cells (Figure 4.16), while U-shaped BVMs that were cultivated with the other protocols exhibited no flattened cells. Therefore, the protocol that utilized the flow rate of $12 \mathrm{~mL} / \mathrm{min}$ was selected as the best of the three protocols proposed in this chapter.

Three issues occurred during this study: (1) an O-ring inside one of the multifunctional bioreactors broke apart during the first day of cultivation, (2) the two-port lid associated with the BVM shown in Figure 4.10 leaked during priming of the two-port lid, and (3) cells appeared fragmented in the BVM shown in Figure 4.10. Air was found in the lumen of the BVM shown in Figure 4.10, and the cells may have died and fragmented as a result of exposure to the air; the air could have entered the complex- 
shaped BVM system via the leak in the two-port lid. Replacement of O-rings in complexshaped BVM systems could ameliorate issues 1 and 2 and is described in Appendix B.

In conclusion, this study showed that cultivation of complex-shaped BVMs using the protocol from current straight BVMs does not cause cells to reach confluence. This study also showed that U-shaped BVMs may exhibit a noticeable loss of cells between the first and fourth days of cultivation whether cultivated at a flow rate of $12 \mathrm{~mL} / \mathrm{min}$ or under no flow at all between the first and fourth days of cultivation. Lastly, this study resulted in the development of a cultivation protocol that yielded a U-shaped BVM with flattened cells; potential alterations to this protocol were therefore proposed, and an alteration was selected and implemented as described in the following chapters. 


\section{Possible Cell Denudation After Stent Deployment in a U-Shaped BVM}

\subsection{Introduction}

This chapter addresses the fourth specific aim of this thesis: determine whether a complex-shaped BVM that was developed using only HUVECs would exhibit denudation of its cell lining after coronary stent deployment.

Two criteria that should be considered when evaluating a potential alteration to the selected protocol from the previous chapter are: (1) the ability of the alteration to cause cells to reach confluence on U-shaped scaffolds and (2) the ability of the alteration to generate U-shaped BVMs capable of exhibiting re-endothelialization of a coronary stent. Two potential alterations are (1) the coating of U-shaped scaffolds with something that improves cell retention on the scaffold and (2) the use of other cell types that exhibit better retention on U-shaped scaffolds than HUVECs.

Whether the proposed alterations potentially meet the first criterion has been determined in previous work. When polytetrafluorethylene (PTFE) is coated with either fibronectin, RGD-containing peptide, laminin, or gelatin, attachment of HUVECs significantly improves [80]. When PTFE is coated with either fibronectin, RGDcontaining peptide, laminin, gelatin, or collagen type I and/or III, proliferation of HUVECs significantly improves [80]. Therefore, coatings affect HUVEC behavior in ways that could lead to confluence. Also, the formation of a thick $(140-\mu \mathrm{m})$ confluent cell lining has resulted from the use of human umbilical vein smooth muscle cells (HUVSMCs) in combination with HUVECs on straight ePTFE scaffolds: a confluent cell lining formed after pressure-sodding HUVSMCs and HUVECs onto straight ePTFE 
scaffolds, cultivating the cell-sodded scaffolds at a flow rate of $1.6 \mathrm{~mL} / \mathrm{min}$ for 1 day, increasing the flow rate by $1.2 \mathrm{~mL} / \mathrm{min}$ every hour until a flow rate of $12 \mathrm{~mL} / \mathrm{min}$ was achieved, and cultivating the cell-sodded scaffolds for 6 more days at a flow rate of 12 $\mathrm{mL} / \mathrm{min}$ [59]. Therefore, a cell type other than HUVECs may result in the formation of a confluent cell lining. These examples show that both proposed alterations may meet the first criterion.

Whether the coating of U-shaped scaffolds potentially meets the second criterion can be determined by examining the effect of stent deployment on the native endothelium. The native endothelium is a monolayer - a cell lining that is only 1 cell thick. When stent deployment was studied in human coronary arteries via SEM, no endothelial cells could be found in the implantation zone during the acute phase $(<6$ weeks) [68]. Stent deployment also disrupts the internal elastic lamina beneath the endothelium [81], exposing smooth muscle cells beneath the internal elastic lamina [82]. Therefore, stent deployment in a U-shaped BVM may completely denude a HUVEC monolayer and thin scaffold coating in the implantation zone, resulting in an implantation zone that consists of simply a bare scaffold exhibiting poor retention of HUVECs. The bare scaffold may then exhibit no re-endothelialization of a coronary stent, suggesting that the stent would exhibit poor re-endothelialization when implanted in a native coronary artery even though the stent may actually re-endothelialize very well in a native artery. Therefore, the coating of U-shaped scaffolds may not meet the second criterion and therefore may not be the best alteration to the selected protocol.

Complete denudation of a HUVEC monolayer in the implantation zone of a Ushaped BVM is only a prediction. But this prediction may be supported by deploying a 
stent in a U-shaped BVM that was developed using one of the three proposed protocols from the previous chapter and by assessing whether denudation occurs in that BVM. Although cell coverage was sparse after cultivation using the three proposed protocols from the previous chapter, cells still remained on all the scaffolds. Deployment of a stent in one of those U-shaped BVMs could result in images that simply show a bare scaffold, suggesting complete denudation of the sparse monolayer of cells, or images that show an intact sparse monolayer, suggesting that stent deployment does not cause complete denudation. Therefore, to determine whether complete denudation actually occurs, a stent was deployed in a U-shaped BVM that was developed using a protocol similar to one of the protocols from the previous chapter. The hypothesis in this case was that a complexshaped BVM that was developed using only HUVECs would exhibit no cell lining after coronary stent deployment. 


\subsection{Materials and Methods}

Two U-shaped BVMs were cultivated in this study using a protocol similar to the protocol from the previous chapter that utilized a flow rate of $0 \mathrm{~mL} / \mathrm{min}$. After cultivation of both BVMs, a stent was deployed in one of the BVMs, while no stent was deployed in the other BVM. Cell culture, BVM cultivation, stent deployment, and evaluation of the BVMs and stent were all performed within a 4-week period. Both BVMs were evaluated using BBI imaging, and the stent was evaluated using SEM imaging.

\subsubsection{Cell Culture}

HUVECs (Lonza) were cultured and expanded in T75 and T225 BD cell culture flasks. Cells were cultured in HUVEC media (Appendix C). The cells were maintained at $37^{\circ} \mathrm{C}$ and $5 \% \mathrm{CO}_{2}$ in a small incubator and monitored using an inverted light microscope. When cells were pressure-sodded onto scaffolds, cells were no more than P8. Enough cells were cultured so that approximately 1.5 million cells $/ \mathrm{cm}^{2}$ were sodded onto each scaffold.

\subsubsection{Scaffold Sterilization, Scaffold Preparation, and Priming}

The 2 scaffolds were prepared and sterilized as described in Section 4.2.2. The scaffolds were denucleated and coated with protein as described in Section 3.2.3. The scaffolds were inserted into multifunctional bioreactors in U-shapes. Scaffolds and reservoirs were primed with conditioning media as described in Section 3.2.4. Two-port lids were then connected to the bioreactors so that the inlets and outlets were correctly connected to the inlets and outlets of the bioreactors. The complex-shaped BVM systems 
were attached to the 8-roller peristaltic pump and primed for 10 minutes at $150 \mathrm{rpm}$ (12 $\mathrm{mL} / \mathrm{min}$ ). All inlets and outlets were closed, and the complex-shaped BVM systems were left in the large incubator.

\subsubsection{Pressure-Sodding of HUVECs}

Cell culture was carried out so that 1 T225 flask of HUVECs was devoted to each scaffold. After the complex-shaped BVM systems were primed, the T225 flasks were removed from the small incubator, and the cells were inspected. The cells were removed from the flasks, centrifuged so that they formed a pellet, and resuspended. Syringes were filled with the cell suspension, the cell suspension was injected transmurally onto each scaffold. All inlets and outlets were closed to prevent media backflow from dislodging cells from scaffold pores. The reservoirs containing conditioning media were replaced with reservoirs containing HUVEC media, the complex-shaped BVM systems were reattached to the peristaltic pump in the large incubator, and the pump was immediately turned on to a setting of $10 \mathrm{rpm}(0.8 \mathrm{~mL} / \mathrm{min})$ to administer transmural flow.

\subsubsection{Cultivation}

Transmural flow at $10 \mathrm{rpm}(0.8 \mathrm{~mL} / \mathrm{min})$ was maintained on both U-shaped BVMs for 1 hour. Then the luminal outlet on both bioreactors was opened, and luminal flow at $10 \mathrm{rpm}(0.8 \mathrm{~mL} / \mathrm{min})$ was maintained for another hour. Luminal flow was then increased to $15 \mathrm{rpm}(1.2 \mathrm{~mL} / \mathrm{min})$ and maintained overnight. After the first day of cultivation, all inlets and outlets of the bioreactors were closed, and the pump was turned off and unplugged. New media was placed in the reservoirs for each BVM every 3 days. 
After $4,5,6,7,9,11$, and 13 days of cultivation, the pump was turned on to $0.8 \mathrm{~mL} / \mathrm{min}$

(10 rpm) for 30 minutes to circulate new media through the lumen of the BVMs. After 14 days of cultivation, one of the BVMs was harvested as described in Section 4.2.6, and a stent was deployed in the other BVM as described in the following section.

\subsubsection{Stent Deployment}

A bare metal stent ( $4.0 \mathrm{~mm} \times 38 \mathrm{~mm}$ coronary stent, Guidant Corporation) was deployed into one of the BVMs after 14 days of BVM cultivation. First, the BVM was detached from the pump and taken into the laminar flow hood, and the lid of the bioreactor was removed and set aside in the hood. The stent was deployed by inserting a stent-loaded balloon catheter into the luminal inlet of the multifunctional bioreactor. The stent was visible through the silicone tubing and through the BVM. The tip of the catheter scraped the inside of the BVM as the tip negotiated the U-shape. The stent was not completely inserted into the BVM — as it did not reach the distal $1 \mathrm{~cm}$ of the BVM. The inflation device was then triggered in an attempt to inflate the balloon, but instead $15 \mathrm{~mL}$ of saline solution was injected directly into the BVM lumen because the catheter was set up incorrectly. The catheter was reconfigured, the balloon was successfully inflated to 8 atm, and the pressure was maintained for 10 seconds. The balloon was then deflated, and the catheter was removed, leaving behind the deployed stent in the BVM. The lid was placed back on the bioreactor, and the complex-shaped BVM system now containing the deployed stent was placed back into the large incubator and connected to the pump. Luminal flow was maintained at $0.8 \mathrm{~mL} / \mathrm{min}(10 \mathrm{rpm})$ for 1.5 hours and was then increased to $1.2 \mathrm{~mL} / \mathrm{min}(15 \mathrm{rpm})$ and allowed to run overnight. The next day, the flow 
rate was increased to $25 \mathrm{rpm}$ and maintained for 1 hour, then increased to $35 \mathrm{rpm}$ and maintained for 1 hour, then increased to $60 \mathrm{rpm}$ and maintained for 1 hour, then increased to $75 \mathrm{rpm}$ and maintained for 1 hour, and then increased to $100 \mathrm{rpm}$ and maintained for 2 days. After the 2 days, the pump was turned off, the complex-shaped BVM system was removed from the pump, and the stented BVM was harvested from the bioreactor and fixed in Histochoice.

\subsubsection{BBI and SEM Imaging}

Both BVMs were cut in half longitudinally. After cutting the stented BVM in half, the stent halves fell out of the BVM halves. All BVM halves were stained with BBI, and en face fluorescent images were captured along the length the BVM halves using a fluorescent microscope. Both stent halves underwent the dehydration protocol for SEM imaging (Appendix K). The stent halves were then dried overnight in a dessicator, and en face images of the stent struts were captured using a scanning electron microscope.

\subsection{Results}

A coronary stent was successfully deployed in a U-shaped BVM that was housed in a multifunctional bioreactor: the catheter was easily fed into the bioreactor, the stent was visible through the silicone tubing and through the U-shaped BVM, and the tip of the catheter was able to negotiate the U-shape of the BVM. When the stent was deployed, the stent straightened out the U-shaped BVM (Figure 5.1). When the stented BVM was cut in half longitudinally, the BVM relaxed away from the more rigid stent (Figure 5.2). 

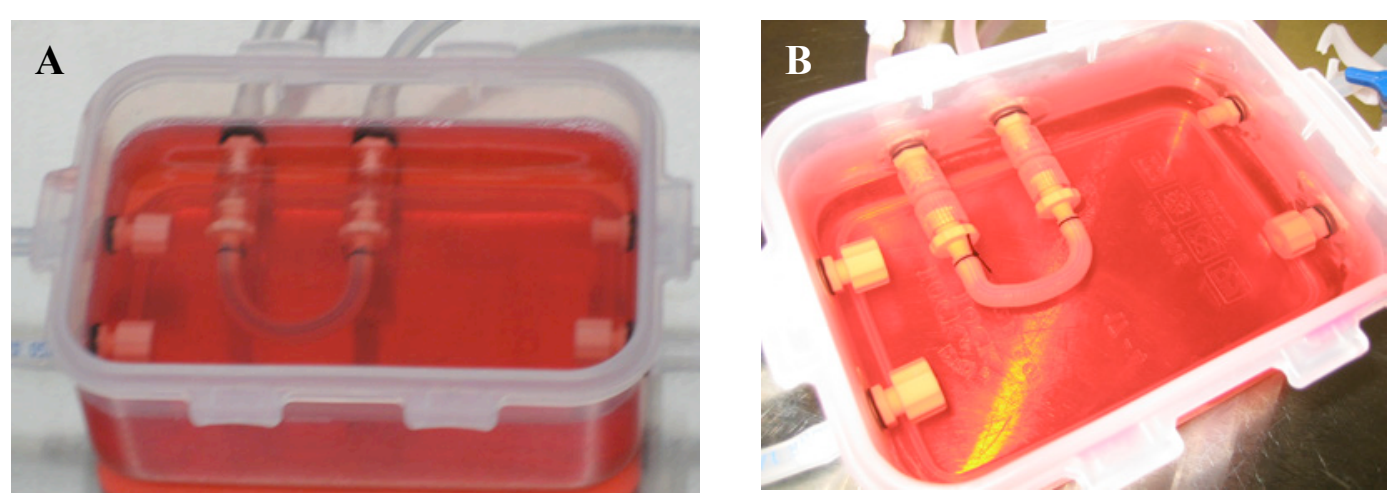

Figure 5.1. Stent deployment in a U-shaped BVM. Before stent deployment, the Ushaped BVM is not straightened out (A). After stent deployment, the stent straightens out the U-shape (B).

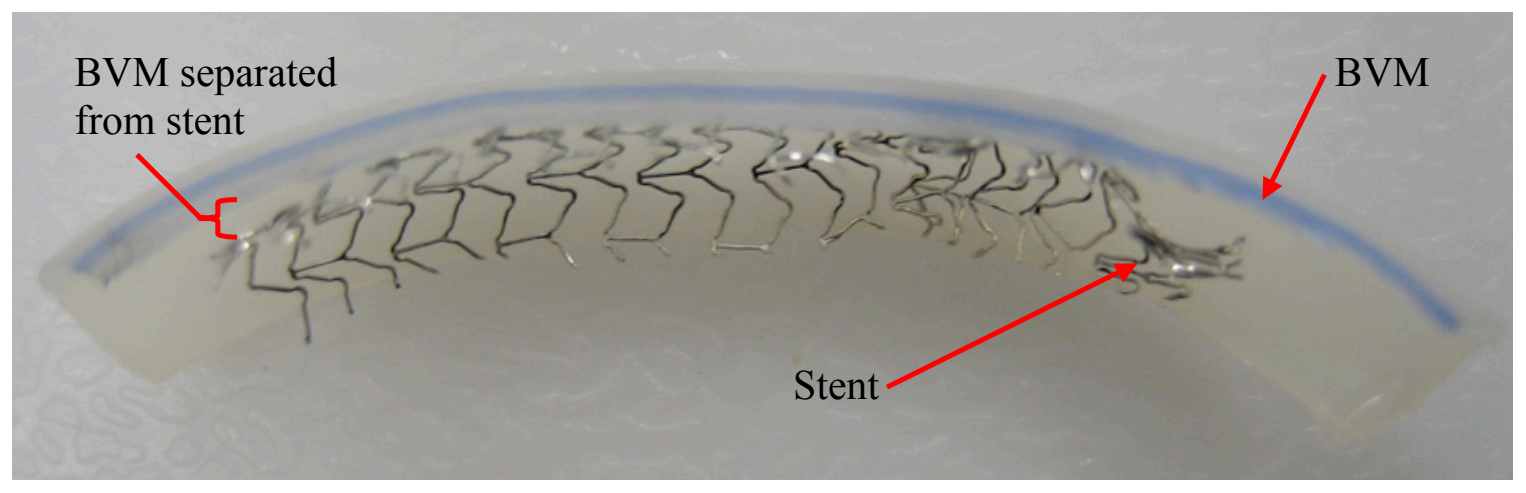

Figure 5.2. Separation of a U-shaped BVM from a rigid coronary stent. The U-shaped BVM relaxed away from the stent halves, and eventually the BVM completely separated from the stent.

$\mathrm{BBI}$ images of the non-stented BVM showed a sparse cell lining similar to the Ushaped BVMs in the previous chapter (Figure 5.3), whereas BBI images of the stented BVM showed no cells (Figure 5.4). SEM images of the stent showed no cell coverage (Figure 5.5). 
Figure 5.3. BBI image of a U-shaped BVM with no implanted stent. A sparse cell lining developed similar to the sparse cell lining of U-shaped BVMs in the previous chapter.

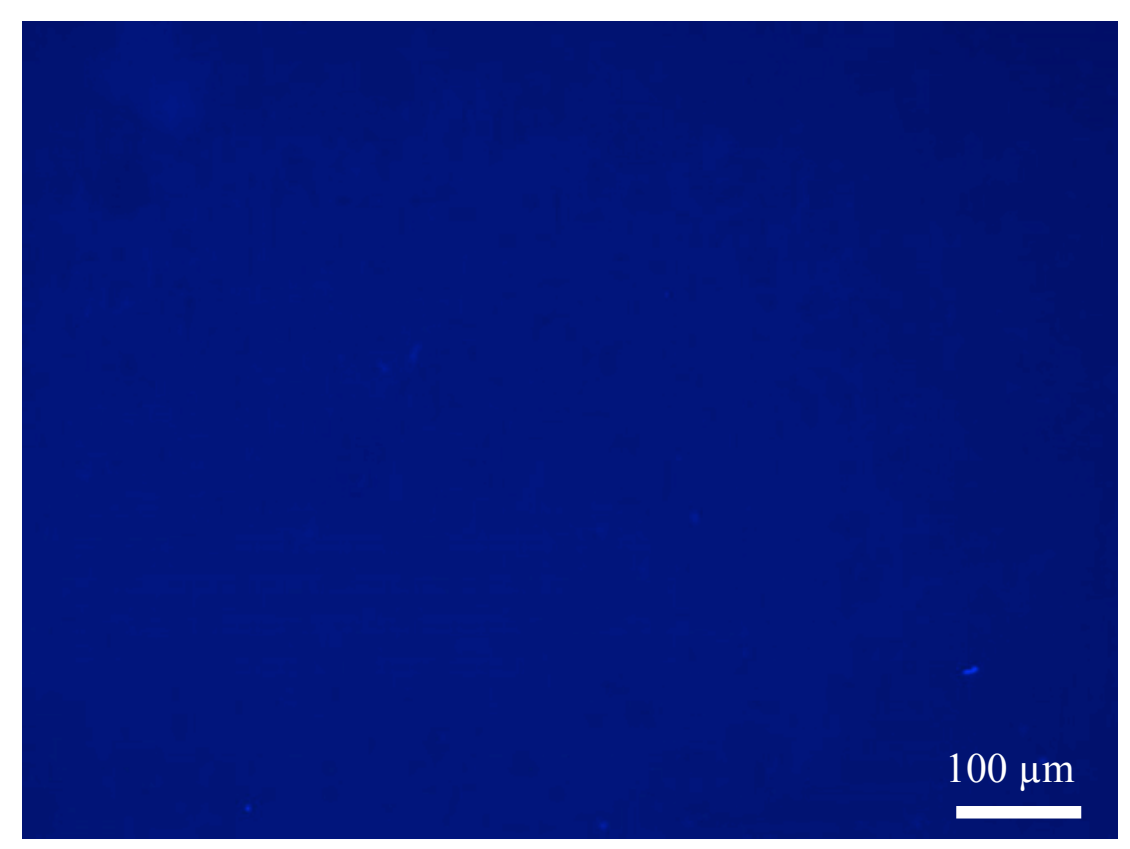

Figure 5.4. BBI image of a U-shaped BVM after stent implantation. No cells are present on the scaffold. 


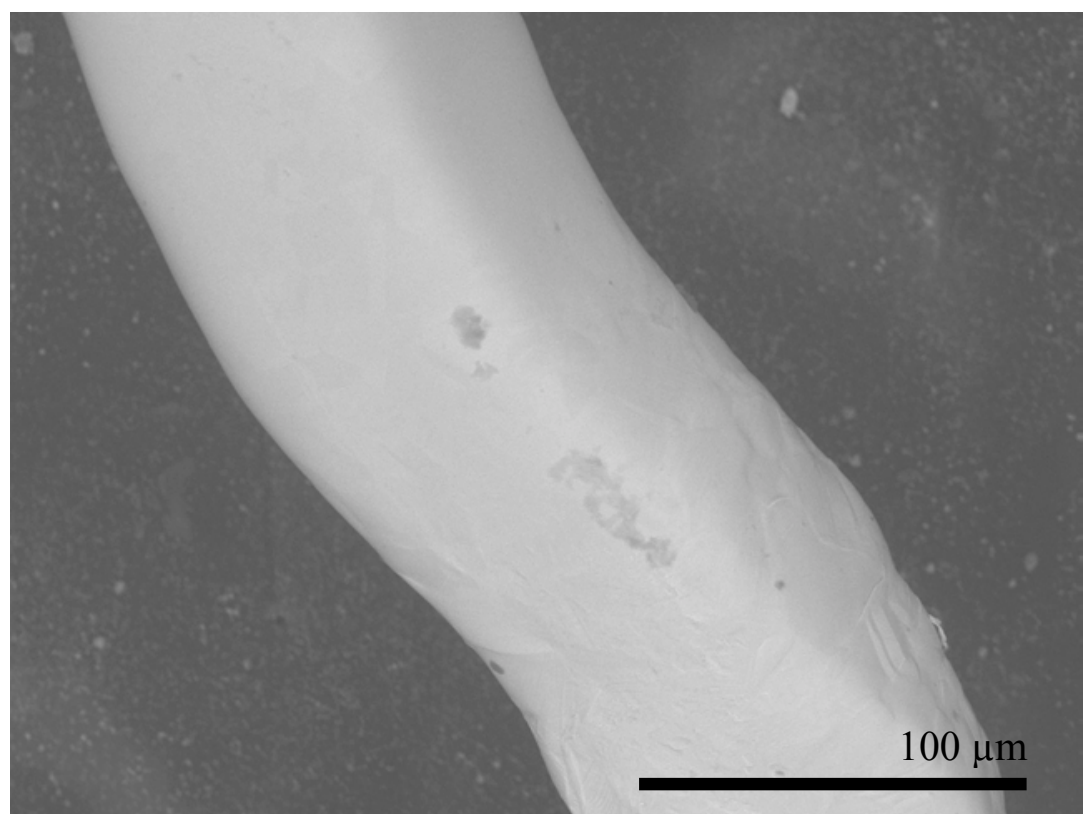

Figure 5.5. Stent strut after implantation in a U-shaped BVM. No cells are visible on the stent strut. All stent struts appeared this way.

\subsection{Discussion and Conclusions}

Deployment of a stent in a U-shaped BVM was performed as described in this chapter to determine whether a stent denudes cells from the luminal surface of a Ushaped BVM. Two U-shaped BVMs were cultivated, and a stent was implanted in one of the BVMs. After harvesting, the non-stented BVM exhibited a sparse cell lining similar to the sparse cell lining of U-shaped BVMs in the previous chapter, whereas the stented BVM exhibited no cell lining of any kind. These findings support the hypothesis that a complex-shaped BVM that was developed using only HUVECs would exhibit no cell lining after coronary stent deployment, although the cause of the cell loss was not determined. Also, no cells appeared on any stent struts, conveying that cells absent from the stented BVM were not located on the stent struts. Therefore, stent deployment may denude cells from a U-shaped BVM. 
This study did not show whether stent deployment actually denuded cells from a U-shaped BVM because the stented BVM was assessed 2 days after stent deployment. To show whether stent deployment actually denuded cells, the stented BVM should have been assessed immediately after stent deployment. But regardless of how stent deployment immediately affected the cell lining, no cell lining regenerated anywhere throughout the stented BVM 2 days after stent deployment. For a U-shaped BVM to convey any information about the tissue response multiple days after stent deployment, cells must be present on the scaffold multiple days after stent deployment. Therefore, the selected protocol from the previous chapter should be altered in a way that allows at least some cells to remain on the scaffold multiple days after stent deployment.

Since stent deployment may denude a monolayer of cells from a U-shaped BVM, possibly resulting in no regeneration of any cell lining multiple days after stent deployment, U-shaped BVMs should be developed to better withstand stent deployment. A cell type that can form multiple cell layers may allow U-shaped BVMs to withstand denudation of a monolayer of cells and then still exhibit re-endothelialization of a stent, whereas a thin layer of coating that improves formation of a HUVEC monolayer on Ushaped scaffolds may be denuded during stent deployment and result in areas of the scaffold that exhibit poor retention of HUVECs, possibly leading to a lack of cell migration onto the stent and therefore an inaccurate portrayal of the stent's ability to reendothelialize. For these reasons, the first alteration that should be made to the selected protocol from the previous chapter should not involve coatings but instead should involve a modification of the cell type that is used to tissue-engineer U-shaped BVMs. 
This study generated other useful information about stent deployment in a Ushaped BVM. A coronary stent was successfully deployed, which validated the complexshaped BVM system for coronary stent testing. Also, during stent deployment, the stent straightened out the U-shape of the BVM, and this straightening also occurs when a coronary stent is deployed in human coronary arteries [60]. The straightening in human coronary arteries creates areas of low endothelial shear stress [61, 62], which may cause the endothelium to re-establish itself more slowly [63]. Therefore, the ability of the Ushaped BVM to mimic the straightening that occurs in native human coronary arteries may yield more accurate assessments of stent re-endothelialization.

A potential issue in this study was that the U-shaped BVM separated from the rigid stent when the stented BVM was cut in half. With future U-shaped BVMs that have confluent cell linings and that are fully capable of testing stents for re-endothelialization, it may be desirable for the stent to remain completely apposed to the BVM wall after being cut in half. Cell coverage on the stent may in fact keep the stent completely apposed to the BVM wall after cutting, but if cell coverage alone does not secure the stent in place, the U-shaped BVM may separate from the rigid stent. This separation could disrupt cell coverage on the stent, and the disrupted cell coverage could be falsely interpreted as the stent's poor ability to re-endothelialize. A possible solution to this separation is to suture the BVM to the stent struts at various locations along the length of the BVM just before cutting so that the two entities do not separate. Also, the separation could be avoided altogether by performing plastic embedding instead of the previously described cutting method. 
In conclusion, this study showed that a U-shaped BVM may exhibit cell denudation after stent implantation, possibly resulting in no regeneration of any cell lining multiple days after stent deployment, this study validated the complex-shaped BVM system for coronary stent testing, and this study showed that a U-shaped BVM mimics the straightening effect that occurs when coronary stents are implanted into human coronary arteries. Because a U-shaped BVM may exhibit cell denudation after stent deployment, a U-shaped BVM will next be tissue-engineered using a cell type that can form multiple cell layers so that the top layer of cells may be denuded while layers beneath remain intact after stent deployment. 


\section{Development of a Confluent Region of Cells in a U-Shaped BVM}

\subsection{Introduction}

This chapter addresses the fifth specific aim of this thesis: develop a confluent region of cells in a U-shaped BVM. Because a complex-shaped BVM may exhibit denudation of its cell lining after coronary stent deployment, the confluent region of cells must be developed using a cell type that is known to form multiple cell layers.

To achieve the fifth specific aim of this thesis, two cell types were used. Although a cell type that forms multiple layers may allow a U-shaped BVM to better withstand stent deployment, cell types that form multiple layers are typically not endothelial cells endothelial cells are contact inhibited and form only monolayers [67]. But throughout this thesis, BVMs have been developed with the goal of modeling re-endothelialization of coronary stents, therefore endothelial cells still must be included in the U-shaped BVM. Therefore, to satisfy the new requirement of being able to withstand denudation of a top layer of cells, but to still satisfy the original requirement of being able to model reendothelialization, two cell types must be used.

Previous work has resulted in the successful development of straight BMVs by sodding two cell types onto a straight scaffold: HUVECs and HUVSMCs. The protocol that was used to develop the dual-sodded straight BVM is described as follows. First, HUVSMCs were pressure-sodded onto a straight ePTFE scaffold, and the straight BVM was transmurally cultivated at a flow rate of $0.8 \mathrm{~mL} / \mathrm{min}(10 \mathrm{rpm})$ for 30 minutes [59]. HUVECs were then pressure-sodded onto the scaffold, and the dual-sodded BVM was transmurally cultivated at a flow rate of $0.8 \mathrm{~mL} / \mathrm{min}(10 \mathrm{rpm})$ for 30 more minutes [59]. 
The dual-sodded BVM was then luminally cultivated at a flow rate of $0.8 \mathrm{~mL} / \mathrm{min}(10$ $\mathrm{rpm})$ for an hour and then at a flow rate of $1.6 \mathrm{~mL} / \mathrm{min}$ (20 rpm) overnight [59]. After dual-sodded straight BVMs were cultivated for just 1 day, they were harvested [59]. Additional dual-sodded straight BVMs were cultivated for more than 1 day, where the flow rate was increased by $1.2 \mathrm{~mL} / \mathrm{min}$ every hour until $12 \mathrm{~mL} / \mathrm{min}(150 \mathrm{rpm})$ was achieved [59]. These dual-sodded straight BVMs were then cultivated for either 3, 5, or 7 days [59]. After all periods of cultivation-1, 3, 5, and 7 days—confluent cell linings formed [59]. After 7 days of cultivation, a multilayer cell lining formed [59]. Because the protocol for developing a dual-sodded straight BVM resulted in formation of a confluent cell lining, the protocol was used to develop a U-shaped BVM with the goal of specifically developing a confluent multilayer region of cells. The hypothesis in this case was that a confluent region of cells would form in a U-shaped BVM.

\subsection{Materials and Methods}

Dual-sodded straight BVMs exhibited total cell coverage after 1 day of cultivation. To be able to compare the results of this study to the dual-sodded straight BVMs, dual-sodded U-shaped BVMs were cultivated for 1 day. Previous HUVEC-only U-shaped BVMs exhibited dense cell coverage after 1 day of cultivation but sparse cell coverage after 4 days of cultivation. To be able to compare the results of this study to previous HUVEC-only U-shaped BVMs, additional dual-sodded U-shaped BVMs were cultivated for 4 days. In total, 6 dual-sodded U-shaped BVMs were cultivated in this study: 3 were cultivated for 1 day, and 3 were cultivated for 4 days. All 6 were cultivated using a protocol almost identical to the protocol described in the introduction of this 
chapter. Cell culture, BVM cultivation, and BVM evaluation were all performed within a 3-week period. A protocol that identifies when various laboratory techniques were used throughout the 3-week period is located in Appendix O. After cultivation, the BVMs were evaluated via histology, fluorescence imaging, and SEM imaging. Also, SEM images of a previous 1-day HUVEC-only U-shaped BVM were captured so that 1-day dual-sodded U-shaped BVMs could be compared to 1-day HUVEC-only U-shaped BVMs.

\subsubsection{Cell Culture}

HUVECs and HUVSMCs were each cultured and expanded in T75 and T225 BD cell culture flasks. HUVECs were cultured in HUVEC media (Appendix C). HUVSMCs were cultured in HUVSMC media using the components and steps described in Appendix M. The cells were maintained at $37^{\circ} \mathrm{C}$ and $5 \% \mathrm{CO}_{2}$ in a small incubator and monitored using an inverted light microscope. When cells were pressure-sodded onto scaffolds, HUVECs were no more than P8, and HUVSMCs were no more than P16. Enough HUVECs were cultured so that approximately 1.5 million cells $/ \mathrm{cm}^{2}$ were sodded onto each scaffold, and enough HUVSMCs were cultured so that approximately 1.5 million cells $/ \mathrm{cm}^{2}$ were sodded onto each scaffold. To identify HUVECs and HUVSMCs via fluorescence microscopy after BVM cultivation, Cell Tracker ${ }^{\mathrm{TM}}$ Red dye (Invitrogen, catalog number C34522) and Cell Tracker ${ }^{\mathrm{TM}}$ Green dye (Invitrogen, catalog number C 7025) were administered to the T225 flasks of HUVECs and HUVSMCs respectively just before the cells were centrifuged. Cell Tracker ${ }^{\mathrm{TM}}$ dyes pass freely through cell 
membranes but then transform into cell-impermeable reaction products. The use of Cell Tracker $^{\mathrm{TM}}$ will be discussed further in later subsections of this chapter.

\subsubsection{Scaffold Sterilization, Scaffold Preparation, and Priming}

6 scaffolds were prepared and sterilized as described in Section 4.2.2. All scaffolds were denucleated and coated with protein as described in Section 3.2.3. The scaffolds were inserted into multifunctional bioreactors in U-shapes. Scaffolds and reservoirs were primed with conditioning media as described in Section 3.2.4. The complex-shaped BVM systems were attached to an 8-roller peristaltic pump and primed for 10 minutes at $150 \mathrm{rpm}(12 \mathrm{~mL} / \mathrm{min})$. All inlets and outlets were closed, and the complex-shaped BVM systems were left in the large incubator.

\subsubsection{Pressure-Sodding of HUVSMCs}

Cell culture was carried out so that 1 T225 flask of HUVSMCs was devoted to each scaffold. While complex-shaped BVM systems were being primed, dual-sodding media was prepared by simply combining HUVSMC media and HUVEC media, resulting in a media that was $25 \%$ HUVSMC media and $75 \%$ HUVEC media; this specific media was used to successfully cultivate previous dual-sodded BVMs [83]. After the complex-shaped BVM systems were primed, the HUVSMC T225 flasks were removed from the small incubator, and the cells were inspected. The cells were removed from the flasks, centrifuged so that they formed a pellet, and resuspended. Syringes were filled with the cell suspension, the cell suspension was injected transmurally onto each scaffold. All inlets and outlets were closed to prevent media backflow from dislodging 
cells from scaffold pores. The reservoirs containing conditioning media were replaced with reservoirs containing dual-sodding media, the complex-shaped BVM systems were reattached to the peristaltic pump in the large incubator, and the pump was immediately turned on to $10 \mathrm{rpm}(0.8 \mathrm{~mL} / \mathrm{min})$ to administer transmural flow for 30 minutes.

\subsubsection{Pressure-Sodding of HUVECs}

During the 30 minutes of transmural flow, the HUVEC T225 flasks were removed from the small incubator, and the cells were inspected. The cells were removed from the flasks, centrifuged so that they formed a pellet, and resuspended. The complex-shaped BVM systems were taken off the peristaltic pump, and the cell suspension was injected transmurally onto each scaffold. All inlets and outlets were closed, the complex-shaped BVM systems were reattached to the peristaltic pump in the large incubator, and the pump was immediately turned on to $10 \mathrm{rpm}(0.8 \mathrm{~mL} / \mathrm{min})$ to administer transmural flow for 30 more minutes.

\subsubsection{Cultivation}

After the 30 additional minutes of transmural flow, the luminal outlet on all bioreactors was opened, and luminal flow at $10 \mathrm{rpm}(0.8 \mathrm{~mL} / \mathrm{min})$ was maintained for 1 hour. Luminal flow was then increased to $20 \mathrm{rpm}(1.2 \mathrm{~mL} / \mathrm{min})$ and maintained overnight. After the overnight cultivation, 3 BVMs were harvested, and the flow rate of the 3 other BVMs was increased by $15 \mathrm{rpm}(1.2 \mathrm{~mL} / \mathrm{min})$ every hour until $150 \mathrm{rpm}(12$ $\mathrm{mL} / \mathrm{min}$ ) was achieved. This flow rate was maintained until the BVMs were harvested after 4 days of cultivation. 


\subsubsection{Histologic Analysis, Fluorescence Imaging, and SEM Imaging}

Imaging of all BVMs was conducted with the goal of assessing whether a confluent multilayer cell lining developed. All BVMs were first cut into the 5 segments shown in Figure 3.6 B. Segments $\mathrm{P}_{2}$ and $\mathrm{D}_{1}$ were histologically analyzed using the protocol located in Appendix K. Segments $\mathrm{P}_{1}, \mathrm{M}$, and $\mathrm{D}_{2}$ were cut in half longitudinally so that each half had one of the blue lines that originally formed the double blue stripe on the ePTFE scaffold. Segments $\mathrm{P}_{1}, \mathrm{M}$, and $\mathrm{D}_{2}$ were analyzed via fluorescence imaging using the protocol located in Appendix G, and segment M was analyzed a second time via SEM imaging using the protocol located in Appendix K.

The Cell TrackerTM dyes were intended to identify HUVSMCs and HUVECs in the cell lining that developed in the BVMs. HUVSMCs were stained with Cell Tracker ${ }^{\mathrm{TM}}$ Green dye (Invitrogen, catalog number C 7025), and HUVECs were stained with Cell Tracker $^{\text {TM }}$ Red dye (Invitrogen, catalog number C34522). Stock solutions of Cell Tracker $^{\mathrm{TM}}$ were prepared by following a previously established protocol [84]; appendix N describes the preparation of Cell Tracker ${ }^{\mathrm{TM}}$ in more detail. Briefly, Cell Tracker ${ }^{\mathrm{TM}}$ powder was diluted to a concentration of $10 \mathrm{mM}$ using DMSO to create a stock solution, and the stock solution was mixed with media to yield a desired concentration of working solution. When white light images of histologic cross-sections were being captured using an Olympus BX41 microscope, 2 fluorescent images of the identical fields of view of the histologic cross-sections were also captured: 1 fluorescent image was captured so that structures fluoresced green, and a second fluorescent image was captured so that structures fluoresced red. The white light image and the 2 fluorescent images were then compared to determine the location of HUVSMCs and HUVECs in the cell lining. 
Segments allotted for BBI imaging were stained with BBI solution. The BBI solution was prepared according to the protocol located in Appendix G. First, $1 \mathrm{~mL}$ of BBI solution was placed in enough foil-wrapped $15-\mathrm{mL}$ conicals so that each segment was allotted a conical. The conicals were labeled with information that identified the specific segment that was stored in that conical. The segments were left in the BBI solution overnight and then imaged according to the protocol located in Appendix G. 4 images were taken of each scaffold segment as described in Figure 3.6 C.

Segments allotted for SEM imaging were first dehydrated in various dilutions of ethanol as described in Appendix K. The segments were then dried overnight in a dessicator. Segments were secured onto the specimen stand of a scanning electron microscope, and 3 SEM images were captured evenly along the length of each segment. Also, SEM images of a previous 1-day HUVEC-only U-shaped BVM were captured.

\subsection{Results}

Multiple histologic cross-sections were obtained from each BVM, and two representative histologic cross-sections were selected for inclusion in this results section: 1 histologic cross-section that represents the BVMs cultivated for 1 day, and 1 histologic cross-section that represents the BVMs cultivated for 4 days. Representative BBI images and SEM images were selected the same way. A dual-sodded U-shaped BVM cultivated for 1 day exhibited a confluent region of cells (Figure 6.1), but all other histologic crosssections, BBI images, and SEM images for all BVMs showed subconfluent cell linings similar to the cell linings in chapter 4 . Figures $6.2-6.7$ show images for dual-sodded Ushaped BVMs cultivated in this study for 1 and 4 days. 


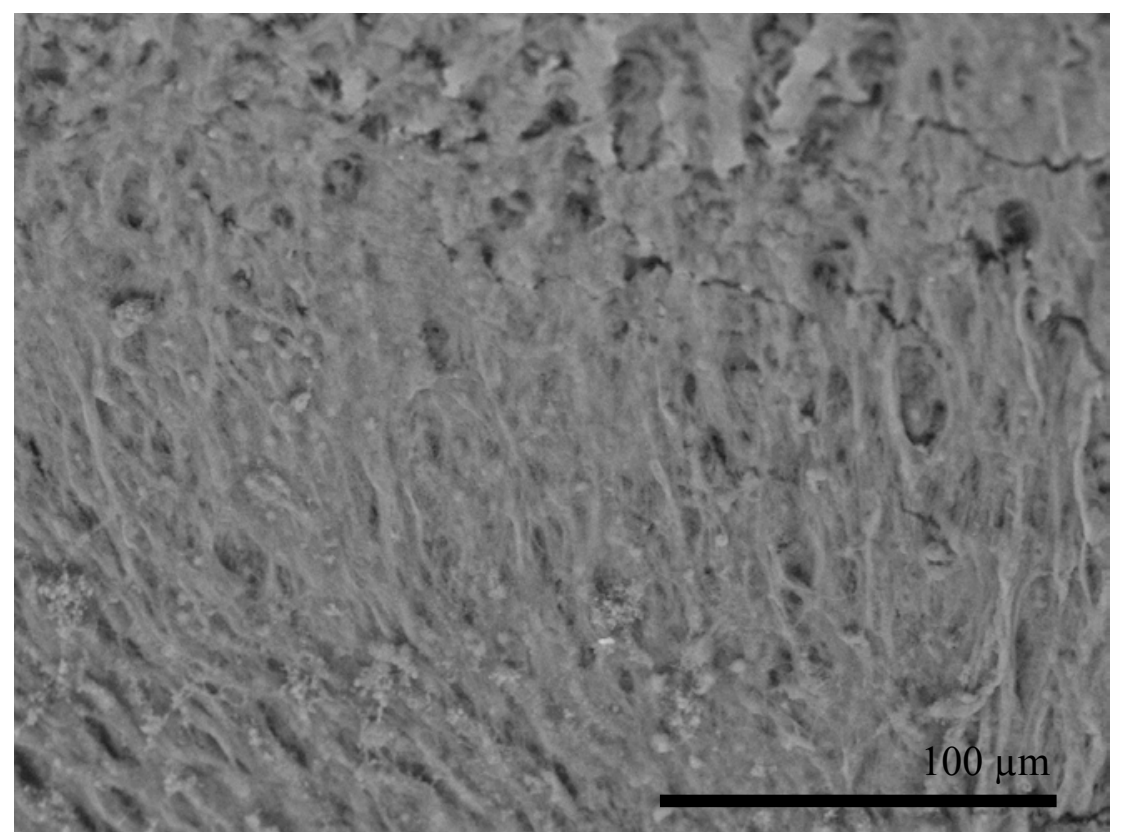

Figure 6.1. Confluent region of cells in a U-shaped BVM cultivated for 1 day. A fully confluent region of cells formed on the outer half of segment $\mathrm{M}$ in a dual-sodded $\mathrm{U}$ shaped BVM. All other SEM images from this BVM and from all other 1-day dualsodded U-shaped BVMs showed cell coverage as depicted in Figure 6.4. 


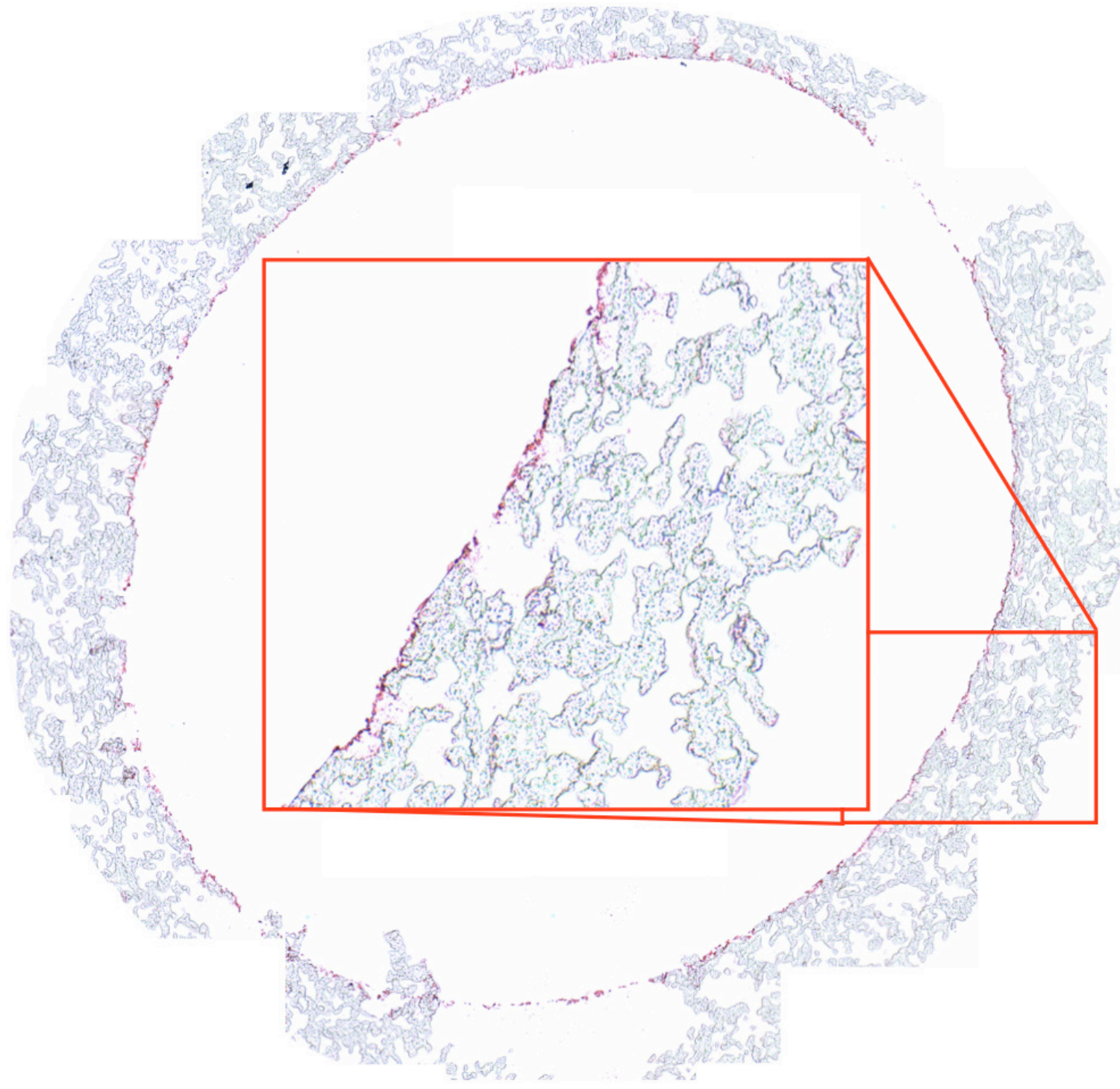

Figure 6.2. Histologic cross-section of a dual-sodded U-shaped BVM cultivated for 1 day. The entire circumference of the cross-section is covered with cells, and cells appear mostly spread-out. 


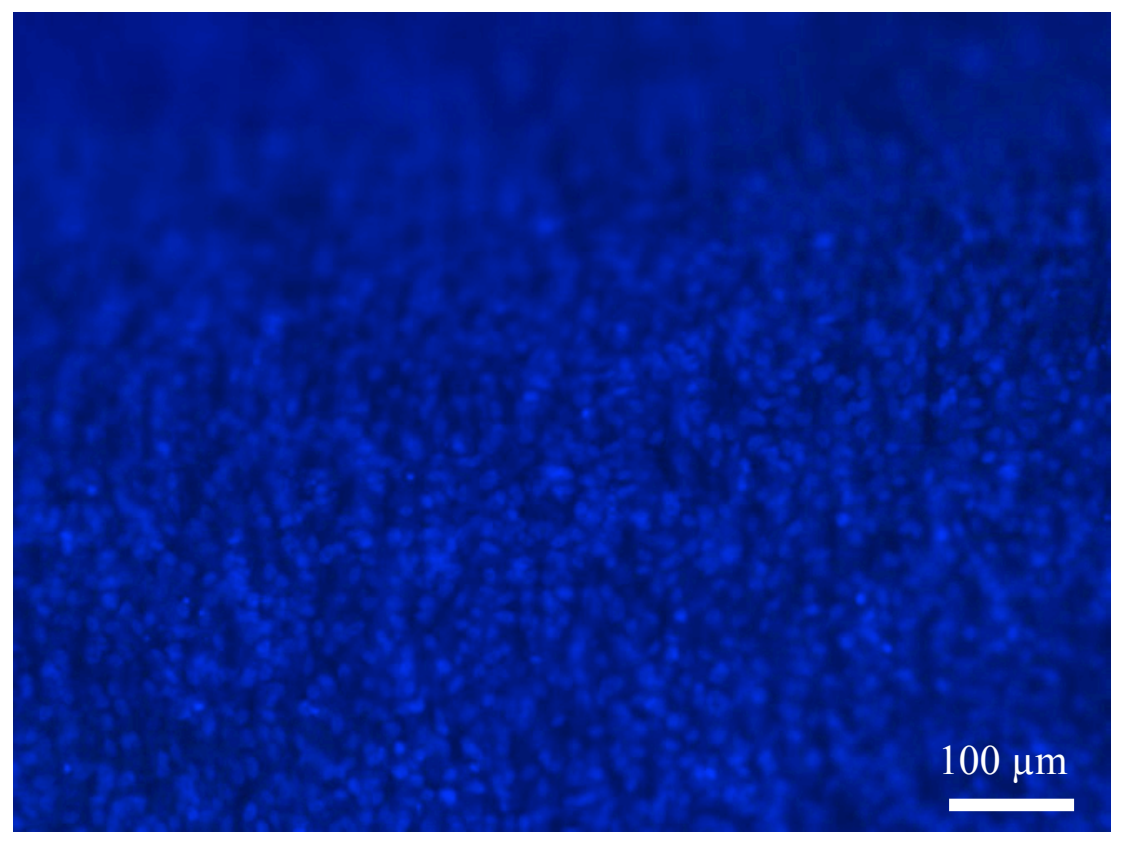

Figure 6.3. BBI image of a dual-sodded U-shaped BVM cultivated for 1 day. Entire image is filled with blue dots—no dark spaces devoid of cells appear between blue dots.

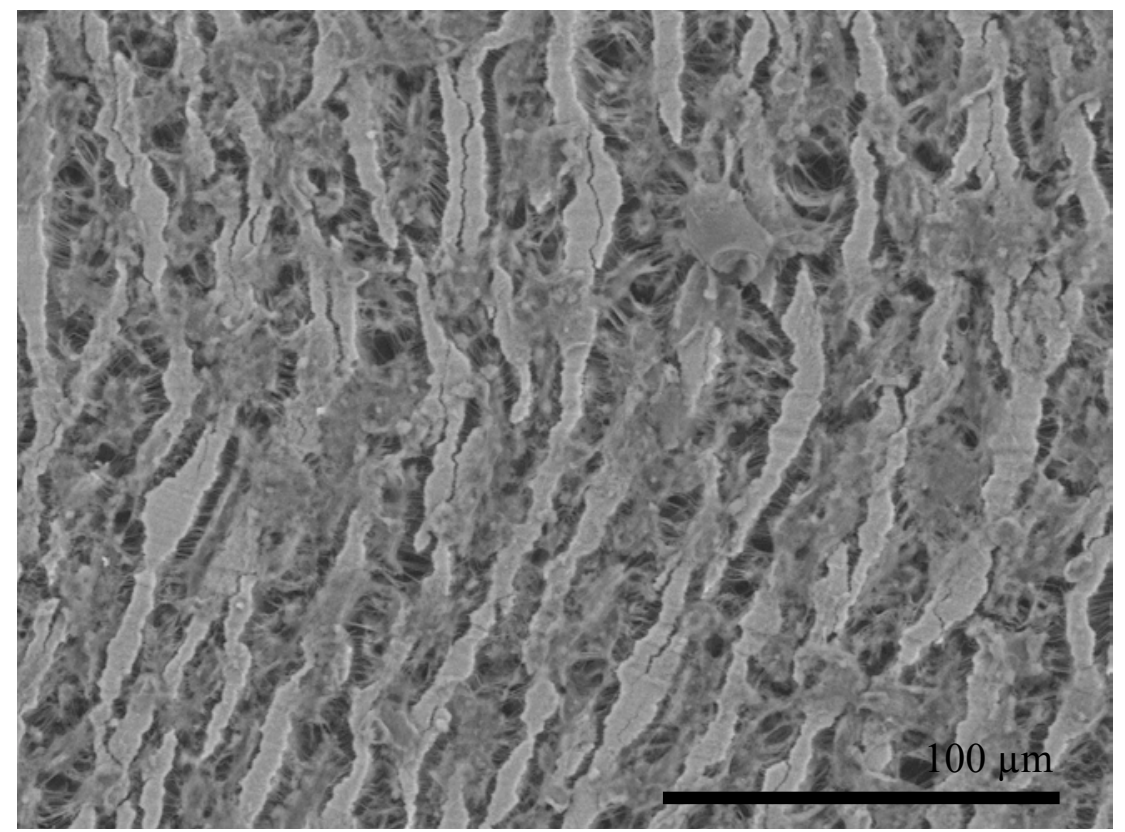

Figure 6.4. SEM image of a dual-sodded U-shaped BVM cultivated for 1 day. Cell coverage is sparse compared to the confluent region of cells in Figure 6.1. 


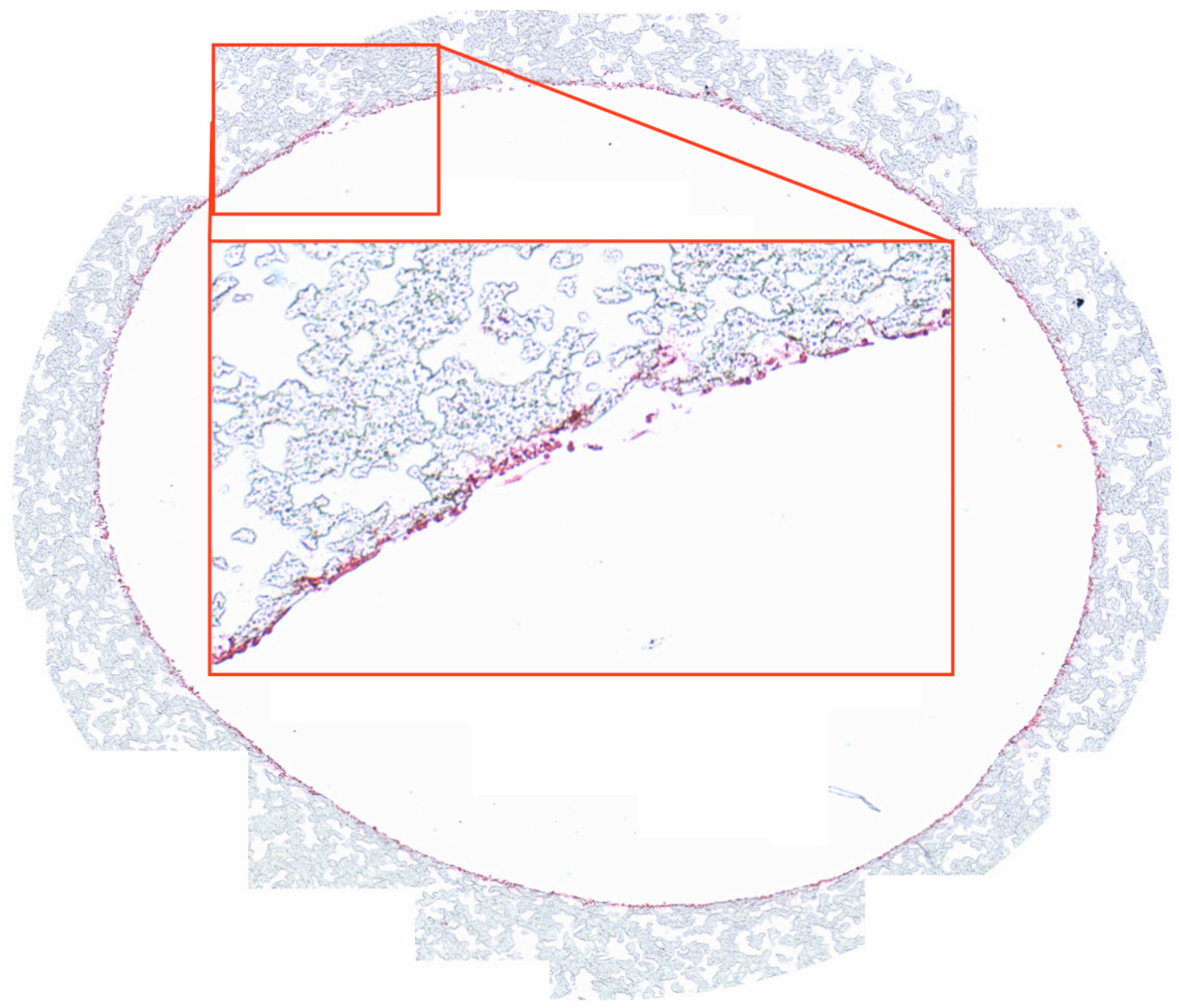

Figure 6.5. Histologic cross-section of a dual-sodded U-shaped BVM cultivated for 4 days at a flow rate of $12 \mathrm{~mL} / \mathrm{min}$. The entire circumference of the cross-section is covered with cells, and many cells appear spread-out. More cell coverage is apparent in this 4-day dual-sodded U-shaped BVM compared to the 4-day HUVEC-only U-shaped BVMs shown in Figures 4.1, 4.7, and 4.13. 


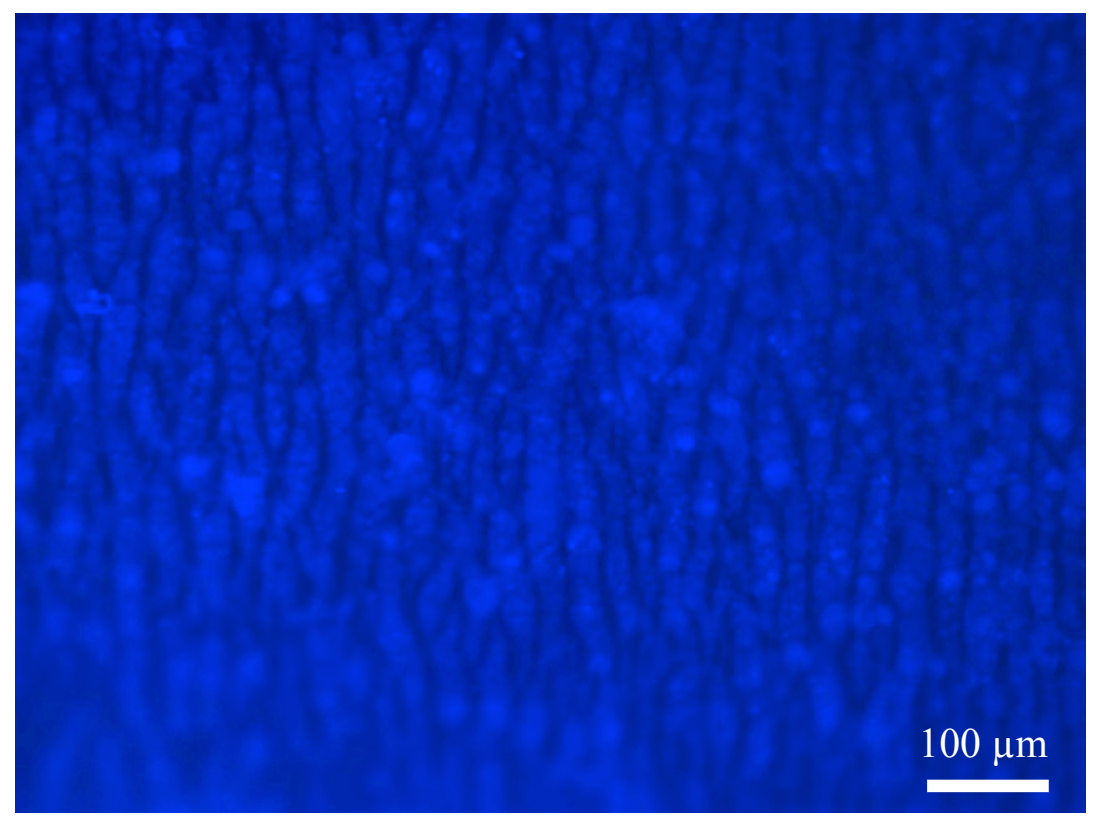

Figure 6.6. BBI image of a dual-sodded U-shaped BVM cultivated for 4 days at a flow rate of $12 \mathrm{~mL} / \mathrm{min}$. Blue dots indicate cells.

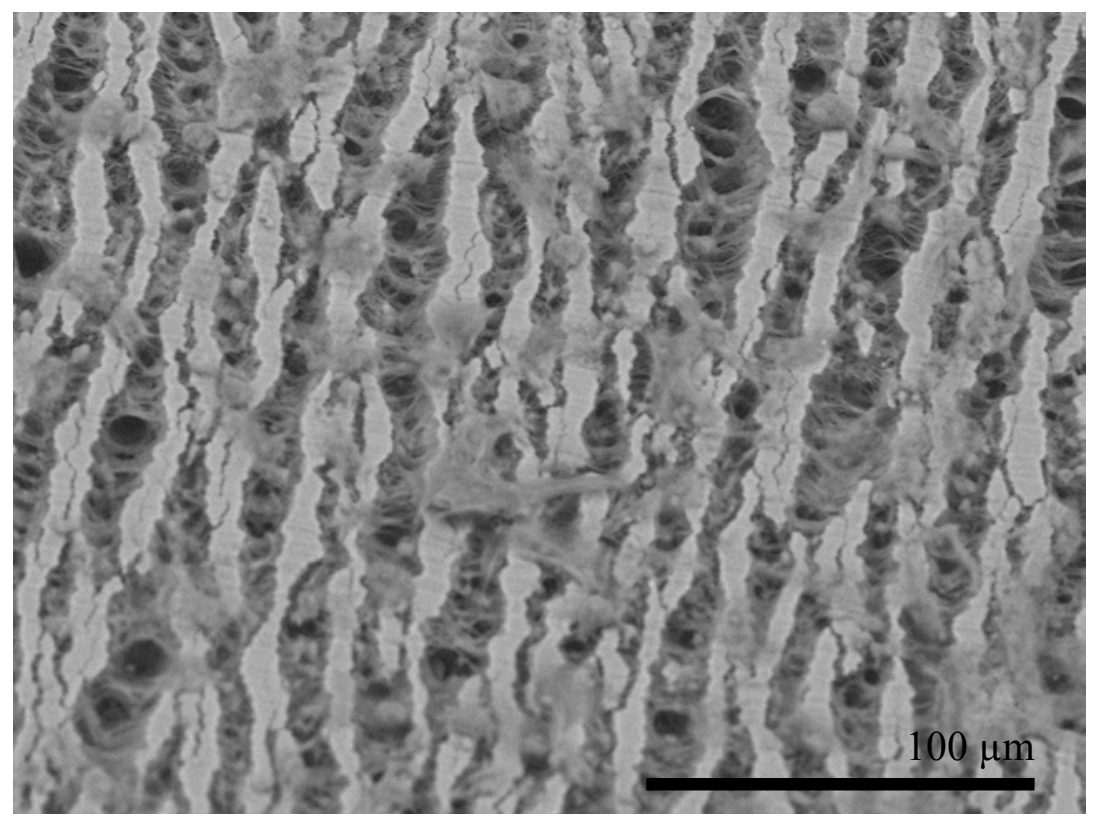

Figure 6.7. SEM image of a dual-sodded U-shaped BVM cultivated for 4 days at a flow rate of $12 \mathrm{~mL} / \mathrm{min}$. More cell coverage appears in this BVM compared to the 4-day HUVEC-only U-shaped BVMs shown in Figures 4.3, 4.9, and 4.15. 
In the fluorescent images that were intended to show HUVSMCs fluorescing green and HUVECs fluorescing red, the structures that fluoresced green also fluoresced red. Therefore, HUVSMCs could not be distinguished from HUVECs in the cell linings of any BVMs (Figure 6.8). 

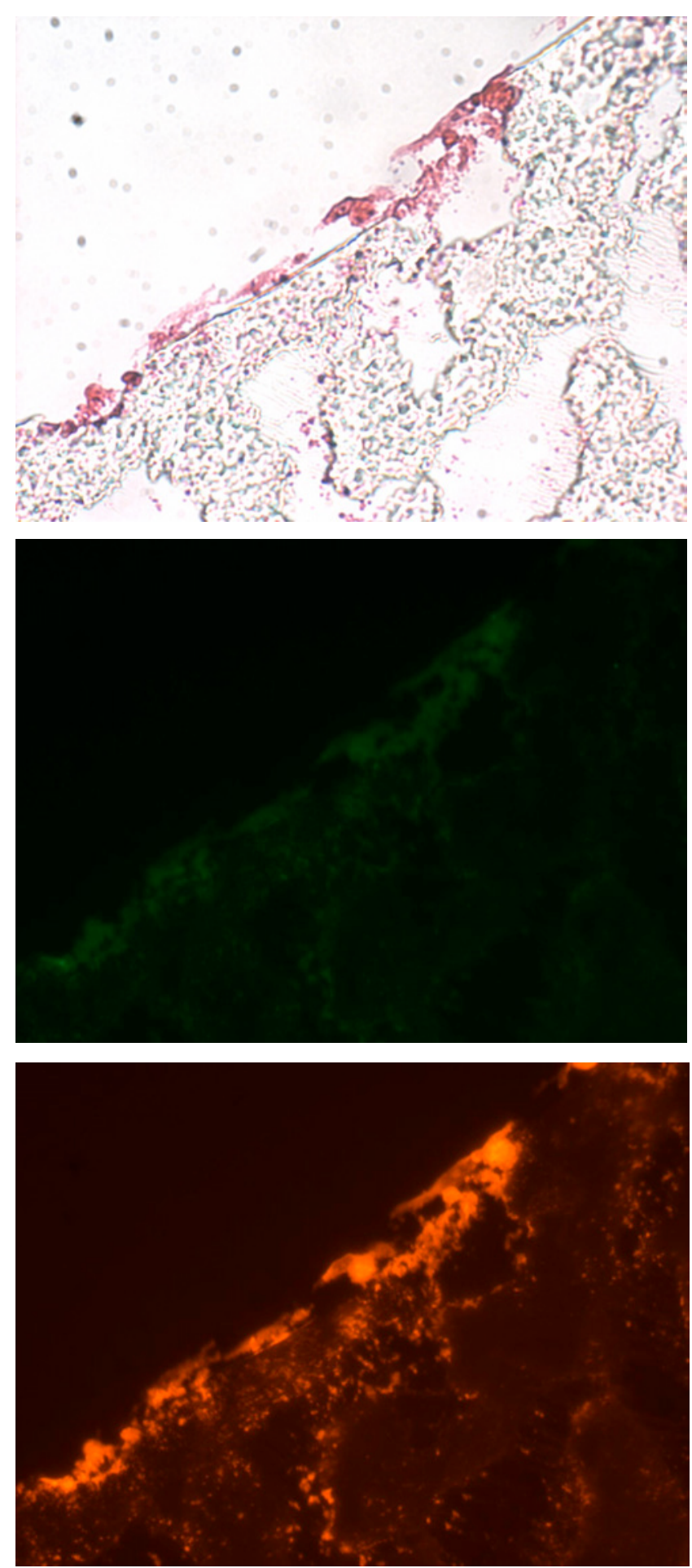

Figure 6.8. Histologic cross-section showing identical structures fluorescing green and red. HUVSMCs were dyed with Cell Tracker ${ }^{\mathrm{TM}}$ Green, and HUVECs were dyed with Cell TrackerTM Red. The top image is a white light image. 


\subsection{Discussion and Conclusions}

A dual-sodded U-shaped BVM was developed as described in this chapter in an attempt to develop a confluent region of cells. After sodding a U-shaped scaffold with HUVSMCs and then HUVECs and cultivating the dual-sodded BVM for 1 day, a confluent region of cells developed on the outer wall of the middle segment. These findings support the hypothesis that a confluent region of cells would form in a U-shaped BVM, although it was not consistent throughout the BVM.

The cell linings on the dual-sodded U-shaped BVMs shown in this chapter were the most confluent cell linings developed in a complex-shaped BVM so far, although the cell linings were still subconfluent. Dual-sodded U-shaped BVMs exhibited more cell coverage than HUVEC-only U-shaped BVMs after both 1 and 4 days of cultivation (Figure 6.9). This was likely because HUVSMCs adhere to the U-shaped scaffold better than HUVECs. But twice as many cells were sodded onto the U-shaped scaffold through the dual-sodding process, therefore dual-sodded U-shaped BVMs also may have exhibited more cell coverage because twice as many cells were sodded onto the scaffold. Future work should determine whether the HUVSMC cell type or the larger number of sodded cells improved cell coverage. 
1-Day

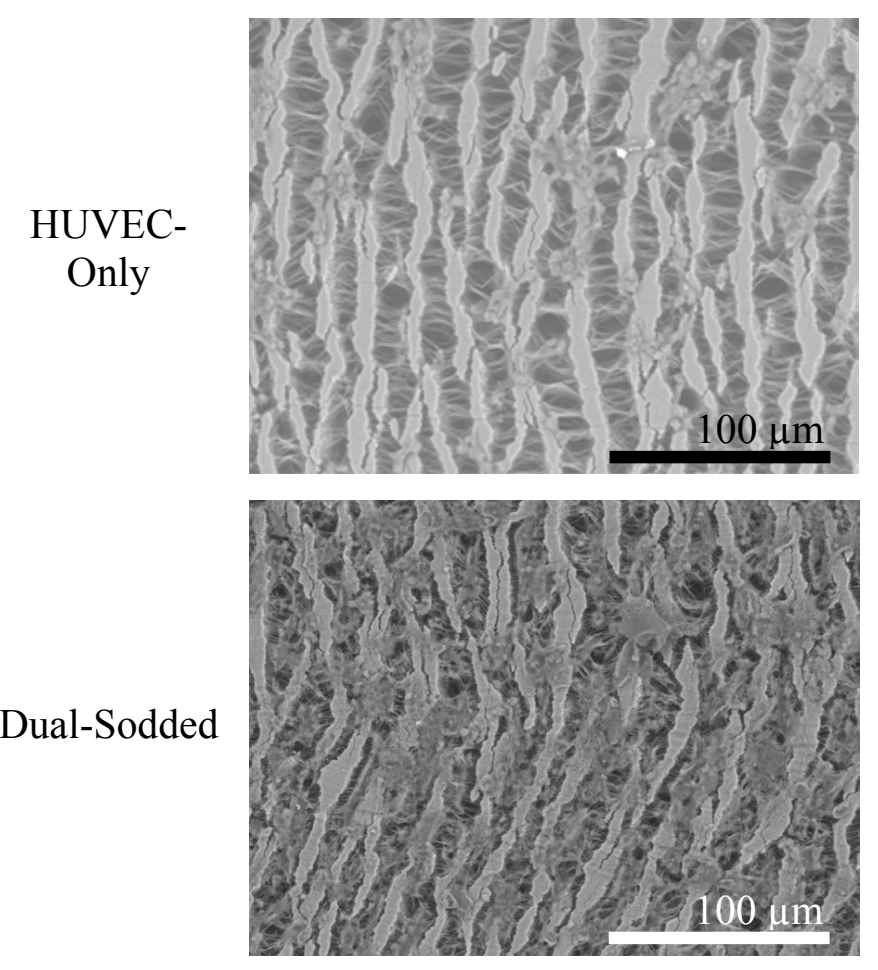

4-Day
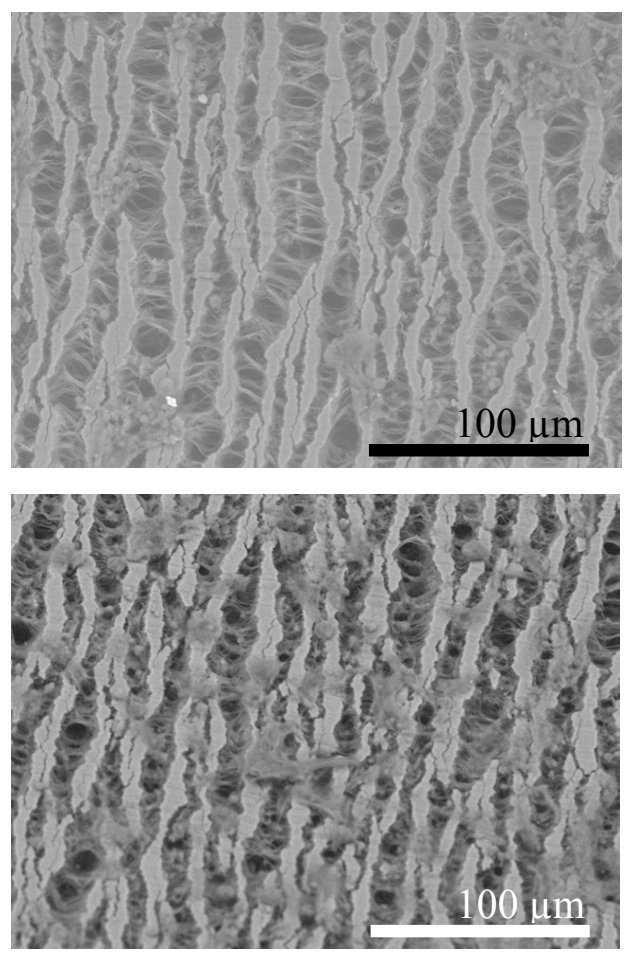

Figure 6.9. Comparison of HUVEC-only U-shaped BVMs to dual-sodded U-shaped

BVMs. Dual-sodded U-shaped BVMs exhibited more cell coverage than HUVEC-only U-shaped BVMs after 1 and 4 days, but cell coverage is still sparse in both.

When an almost identical dual-sodding protocol is used to cultivate dual-sodded straight BVMs and dual-sodded U-shaped BVMs, the dual-sodded straight BVMs exhibit confluent cell linings after 1 day of cultivation, while the dual-sodded U-shaped BVMs do not. The same issues(s) that resulted in a lack of confluent cell linings in HUVEC-only U-shaped BVMs may have also resulted in a lack of confluent cell linings in dual-sodded U-shaped BVMs. One of these issues may be that the unique flow in a U-shaped scaffold that results in higher wall shear stress may push more cells off the scaffold than the flow 
in a straight scaffold that results in lower wall shear stress, resulting in more spaces of bare scaffold between cells on the U-shaped scaffold.

An issue with the Cell Tracker ${ }^{\mathrm{TM}}$ occurred during this study: although HUVSMCs were dyed with Cell Tracker ${ }^{\mathrm{TM}}$ Green and HUVECs were dyed with Cell Tracker ${ }^{\mathrm{TM}}$ Red, the structures that fluoresced green also fluoresced red. A potential explanation for this issue is that the BVMs underwent fixation, processing, paraffin-embedding, and staining for histology, and this may have affected the Cell Tracker ${ }^{\mathrm{TM}}$ in a way that caused the outcome in Figure 6.8. Therefore, future work should be carried out to improve Cell Tracker $^{\mathrm{TM}}$ performance in BVMs that have undergone histology procedures.

In conclusion, after sodding a U-shaped scaffold with HUVSMCs and then HUVECs and cultivating the dual-sodded BVM, a confluent region of cells did develop in one area of the dual-sodded U-shaped BVM, but this result was inconsistent. Therefore, it is possible that further development of this protocol will result in a confluent lining of cells in a U-shaped BVM. 


\section{Discussion and Conclusions}

\subsection{Review of the Purpose of Complex-Shaped BVMs}

The complex geometries of coronary arteries are prone to lethal plaque formations that cause CHD. As a result, CHD is the leading cause of death in the United States, and new coronary devices are constantly under development to identify and treat this disease. The FDA requires that these devices be tested, but current in vitro and in vivo testing methods have drawbacks. In response to these drawbacks, BVMs were developed in straight geometries and have been used to identify stents that re-endothelialize better than other stents. But in certain cases where the complex geometries of coronary arteries may negatively affect coronary device performance, the FDA has specifically recommended that coronary devices be tested in complex-shaped simulated vessels. Therefore, BVMs should be developed in the complex geometries of coronary arteries.

\subsection{Summary of Thesis Work}

The work in this thesis describes the development of complex-shaped scaffolds and bioreactors, the development of complex-shaped BVMs with cells located throughout all regions of the BVMs, and the development of a U-shaped BVM with a confluent region of cells.

The first aim of this work was to design, construct, and validate complex-shaped scaffolds and bioreactors. The hypothesis in this case was that complex-shaped scaffolds could be designed and constructed, that bioreactors capable of housing the new scaffolds could be designed and constructed, and that cells would deposit onto the new scaffolds 
inside the new bioreactors. Complex-shaped scaffolds were constructed, bioreactors capable of housing the new scaffolds were constructed, and cells deposited on the new scaffolds. These results supported the hypothesis.

The second specific aim was to deposit cells throughout the entire inner surface of complex-shaped scaffolds. The hypothesis in this case was that, after 1 day of cultivation of complex-shaped BVMs, no statistically significant differences would exist between the cell densities along the length of the BVMs, between the cell densities in the inner and outer halves of the BVMs, or between the cell densities in the top and bottom halves of the BVMs. Although statistically significant differences did exist between 5 regions along the length of L-shaped BVMs, between the inner and outer halves of L-shaped BVMs, and between 5 regions along the length of U-shaped BVMs, cells did deposit with average or median densities between 250,000 cells $/ \mathrm{cm}^{2}$ and $350,000 \mathrm{cells} / \mathrm{cm}^{2}$ throughout all regions of L- and U-shaped BVMs. Results did not support the hypothesis, but results did confirm that cells can deposit throughout all regions of complex-shaped BVMs at densities that are approximately the same order of magnitude as endothelial cell densities in various native blood vessels.

The third specific aim was to determine whether HUVECs would reach confluence on a complex-shaped scaffold using a cultivation protocol similar to the straight-BVM cultivation protocol. The hypothesis in this case was that after deposition of HUVECs onto U-shaped scaffolds and cultivation similar to the straight-BVM protocol, cells would reach confluence. Cells did not reach confluence using three different cultivation protocols, therefore results did not support the hypothesis. But the 
protocol utilizing the constant flow rate of $12 \mathrm{~mL} / \mathrm{min}$ did result in development of a Ushaped BVM with flattened cells.

After none of the proposed cultivation protocols resulted in development of any region of confluence in a U-shaped BVM, the best proposed cultivation protocol was selected, and multiple alterations to the protocol were proposed. Alterations that focused on coating the U-shaped scaffold or developing a monolayer of endothelial cells on the U-shaped scaffold were not desirable because it was suspected that stent deployment would denude a thin coating or thin cell lining from the U-shaped scaffold. But because denudation after stent deployment in a complex-shaped BVM was only a suspicion, the fourth specific aim of this thesis was to determine whether a complex-shaped BVM developed using only HUVECs would exhibit denudation of its cell lining after coronary stent deployment. The hypothesis in this case was that a complex-shaped BVM that was developed using only HUVECs would exhibit no cell lining after coronary stent deployment. A U-shaped BVM did exhibit no cell lining after coronary stent deployment, therefore the results supported the hypothesis, and potential alterations that involved thin coatings or thin cell linings were avoided. The stent deployment study also validated the complex-shaped BVM system for coronary stent testing and revealed the U-shaped BVM's important ability to mimic the straightening effect that occurs when coronary stents are implanted in human coronary arteries.

The fifth specific aim of this thesis was to develop a confluent region of cells in a U-shaped BVM. Because a complex-shaped BVM exhibited possible denudation of its cell lining after coronary stent deployment, the confluent region of cells had to be developed using a cell type that was known to form multiple cell layers so that the cells 
could eventually be cultivated into a multilayer that would withstand stent deployment better than a monolayer. The hypothesis in this case was that a confluent region of cells would form in a U-shaped BVM. A confluent region of cells did develop in one area of a U-shaped BVM, although this result was inconsistent. Therefore, results supported the hypothesis. These results revealed that a confluent region of cells can develop in a Ushaped BVM even though a U-shaped scaffold exhibits more than 7 times the wall shear stress of a straight scaffold. Overall, the results from all five aims support the hypothesis that a BVM could be developed in the complex geometries of coronary arteries.

\subsection{Limitations and Future Work}

Although this thesis has culminated with the development of a confluent region of cells in a U-shaped BVM, a confluent cell lining throughout all regions of a complexshaped BVM is necessary to predict re-endothelialization of a coronary stent. Therefore, future work should focus on the development of a confluent cell lining throughout all regions of a U-shaped BVM. Future steps may involve sodding U-shaped scaffolds with more cells, sodding a single U-shaped scaffold multiple days in a row so that cells can fall off the scaffold but be replaced with new cells, or allowing HUVSMCs to develop into a thick cell lining over a longer period and then sodding HUVECs on top of the thick HUVSMC lining. No matter what alterations are made to the protocol for the tissue engineering of complex-shaped BVMs, a main criterion that should be considered when evaluating potential alterations is the ability of the alteration to withstand stent deployment and still function usefully after stent deployment. 
Other future work on complex-shaped BVMs could involve the complete development of BVMs in the other proposed geometries. Complete development includes validation of the bifurcated scaffolds and the bioreactor capable of housing only Yshaped scaffolds, quantification of cell deposition on S-, T-, and Y-shaped scaffolds, development of a confluent cell lining on all the complex-shaped scaffolds, successful stent deployment in fully functional complex-shaped BVMs, and a repeatable, consistent BVM tissue response to coronary stents.

\subsection{Conclusions}

In conclusion, complex-shaped scaffolds and bioreactors were fully developed, complex-shaped BVMs were developed with cells located throughout all regions of the BVMs, and a U-shaped BVM was developed with a confluent region of cells. In addition to preclinically testing coronary stents, potential applications of complex-shaped BVMs include preclinical testing of drug-delivery systems, coronary imaging modalities, and other coronary technologies. Complex-shaped BVMs may eventually improve the accuracy of preclinical coronary device testing and result in the development of improved coronary devices, which could directly benefit CHD patients. 


\section{References}

1. Lloyd-Jones D, Adams RJ, Brown TM, Carnethon M, Dai S, De Simone G, et al. Heart disease and stroke statistics--2010 update: a report from the American Heart Association. Circulation 2009 Feb 23;121(7):e46-e215.

2. Naghavi M, Libby P, Falk E, Casscells SW, Litovsky S, Rumberger J, et al. From vulnerable plaque to vulnerable patient: a call for new definitions and risk assessment strategies: Part I. Circulation 2003 Oct 7;108(14):1664-1672.

3. Smith SC, Jr., Dove JT, Jacobs AK, Kennedy JW, Kereiakes D, Kern MJ, et al. ACC/AHA guidelines for percutaneous coronary intervention (revision of the 1993 PTCA guidelines)-executive summary: a report of the American College of Cardiology/American Heart Association task force on practice guidelines (Committee to revise the 1993 guidelines for percutaneous transluminal coronary angioplasty) endorsed by the Society for Cardiac Angiography and Interventions. Circulation 2001 Jun 19;103(24):3019-3041.

4. Jakob M, Spasojevic D, Krogmann ON, Wiher H, Hug R, Hess OM. Tortuosity of coronary arteries in chronic pressure and volume overload. Cathet Cardiovasc Diagn 1996 May;38(1):25-31.

5. Loukas M, Groat C, Khangura R, Owens DG, Anderson RH. The Normal and Abnormal Anatomy of the Coronary Arteries. Clinical Anatomy 2009 Nov;22:114128.

6. He X, Ku DN. Pulsatile flow in the human left coronary artery bifurcation: average conditions. J Biomech Eng 1996 Feb;118(1):74-82. 
7. Ku DN. Blood flow in arteries. Annual Review of Fluid Mechanics 1997;29(1):399434.

8. Myers JG, Moore JA, Ojha M, Johnston KW, Ethier CR. Factors Influencing Blood Flow Patterns in the Human Right Coronary Artery. Annals of Biomedical Engineering 2001;29(2):109-120.

9. Chatzizisis YS, Coskun AU, Jonas M, Edelman ER, Feldman CL, Stone PH. Role of endothelial shear stress in the natural history of coronary atherosclerosis and vascular remodeling: molecular, cellular, and vascular behavior. J Am Coll Cardiol 2007 Jun 26;49(25):2379-2393.

10. Buchanan JR, Kleinstreuer C, Truskey GA, Lei M. Relation between non-uniform hemodynamics and sites of altered permeability and lesion growth at the rabbit aortoceliac junction. Atherosclerosis 1999;143:27-40.

11. Malek AM, Alper SL, Izumo S. Hemodynamic shear stress and its role in atherosclerosis. JAMA 1999 Dec 1;282(21):2035-2042.

12. Feldman CL, Chatzizisis YS, Coskun AU, Koskinas KC, Naghavi M, Stone PH. Vulnerable anatomy; the role of coronary anatomy and endothelial shear stress in the progression and vulnerability of coronary artery lesions: is anatomy destiny? Asymptomatic Atherosclerosis: Pathophysiology, Detection and Treatment 2010:495506.

13. Insull W, Jr. The pathology of atherosclerosis: plaque development and plaque responses to medical treatment. Am J Med 2009 Jan;122(1 Suppl):S3-S14. 
14. Miller JM, Rochitte CE, Dewey M, Arbab-Zadeh A, Niinuma H, Gottlieb I, et al. Diagnostic performance of coronary angiography by 64-row CT. N Engl J Med 2008 Nov 27;359(22):2324-2336.

15. Jasmin L. Percutaneous Transluminal Coronary Angioplasty (PTCA). MedlinePlus by the National Institutes of Health. 2010 [cited 2011; Available from: http://www.nlm.nih.gov/medlineplus/ency/anatomyvideos/000096.htm

16. Derian W, Hertsberg A. Acute myocardial infarction from simultaneous total occlusion of the left circumflex and right coronary artery. A case report. Int J Cardiol 2007 Jul 10;119(2):e65-67.

17. Garcia-Garcia HM, Costa MA, Serruys PW. Imaging of coronary atherosclerosis: intravascular ultrasound. Eur Heart J 2010 Oct;31(20):2456-2469.

18. Sharma S, Christopoulos C, Kukreja N, Gorog DA. Local drug delivery for percutaneous coronary intervention. Pharmacol Ther 2011 Mar;129(3):260-266.

19. National Heart, Lung, and Blood Institute (NHLBI). How is Coronary Angioplasty Done? 2012 [cited April 17, 2012; Available from: http://www.nhlbi.nih.gov/health/health-topics/topics/angioplasty/howdone.html

20. Schillinger M, Exner M, Mlekusch W, Haumer M, Sabeti S, Ahmadi R, et al. Restenosis after femoropopliteal PTA and elective stent implantation: predictive value of monocyte counts. J Endovasc Ther 2003 Jun;10(3):557-565.

21. Butany J, Carmichael K, Leong SW, Collins MJ. Coronary artery stents: identification and evaluation. J Clin Pathol 2005 Aug;58(8):795-804.

22. Nawarskas JJ, Osborn LA. Paclitaxel-eluting stents in coronary artery disease. Am J Health Syst Pharm 2005 Nov 1;62(21):2241-2251. 
23. Schofield PM. Indications for percutaneous and surgical revascularisation: how far does the evidence base guide us? Heart 2003 May;89(5):565-570.

24. Sanders M. Angiographic changes thirty minutes following percutaneous transluminal coronary angioplasty. Angiology $1985 \mathrm{Jul} ; 36(7): 419-424$.

25. Nobuyoshi M, Kimura T, Ohishi H, Horiuchi H, Nosaka H, Hamasaki N, et al. Restenosis after percutaneous transluminal coronary angioplasty: pathologic observations in 20 patients. J Am Coll Cardiol 1991 Feb;17(2):433-439.

26. Waller BF. "Crackers, breakers, stretchers, drillers, scrapers, shavers, burners, welders and melters"--the future treatment of atherosclerotic coronary artery disease? A clinical-morphologic assessment. J Am Coll Cardiol 1989 Apr;13(5):969-987.

27. Zargham R. Preventing restenosis after angioplasty: a multistage approach. Clin Sci (Lond) $2008 \mathrm{Feb} ; 114(4): 257-264$.

28. Gibbons GH, Dzau VJ. The emerging concept of vascular remodeling. N Engl J Med 1994 May 19;330(20):1431-1438.

29. Herdeg C, Gohring-Frischholz K, Haase KK, Geisler T, Zurn C, Hartmann U, et al. Catheter-based delivery of fluid paclitaxel for prevention of restenosis in native coronary artery lesions after stent implantation. Circ Cardiovasc Interv 2009 Aug;2(4):294-301.

30. Palmaz JC, Sibbitt RR, Reuter SR, Tio FO, Rice WJ. Expandable intraluminal graft: a preliminary study. Work in progress. Radiology 1985 Jul;156(1):73-77.

31. Cardinal KO. Development and Utilization of a Tissue Engineered Blood Vessel Mimic to Assess the Neointimal Response to Intravascular Stents. Tucson: University of Arizona; 2007. 
32. Kandzari DE, Tcheng JE, Zidar JP. Coronary artery stents: evaluating new designs for contemporary percutaneous intervention. Catheter Cardiovasc Interv 2002 Aug;56(4):562-576.

33. Indolfi C, Mongiardo A, Curcio A, Torella D. Molecular mechanisms of in-stent restenosis and approach to therapy with eluting stents. Trends Cardiovasc Med 2003 May;13(4):142-148.

34. Huang Y, Venkatraman SS, Boey FY, Umashankar PR, Mohanty M, Arumugam S. The short-term effect on restenosis and thrombosis of a cobalt-chromium stent eluting two drugs in a porcine coronary artery model. J Interv Cardiol 2009 Oct;22(5):466478.

35. Byrne RA, Kastrati A, Tiroch K, Schulz S, Pache J, Pinieck S, et al. 2-year clinical and angiographic outcomes from a randomized trial of polymer-free dual drug-eluting stents versus polymer-based Cypher and Endeavor [corrected] drug-eluting stents. J Am Coll Cardiol 2010 Jun 8;55(23):2536-2543.

36. Colombo A, Karvouni E. Biodegradable stents: "fulfilling the mission and stepping away". Circulation 2000 Jul 25;102(4):371-373.

37. Ormiston JA, Serruys PW, Regar E, Dudek D, Thuesen L, Webster MW, et al. A bioabsorbable everolimus-eluting coronary stent system for patients with single denovo coronary artery lesions (ABSORB): a prospective open-label trial. Lancet 2008 Mar 15;371(9616):899-907.

38. Wykrzykowska JJ, Onuma Y, Serruys PW. Vascular restoration therapy: the fourth revolution in interventional cardiology and the ultimate "rosy" prophecy. EuroIntervention 2009 Dec 15;5 Suppl F:F7-8. 
39. United States Food and Drug Administration (FDA). Guidance for Industry: Coronary Drug-Eluting Stents_-Nonclinical and Clinical Studies. 2008. p. 35 and 39.

40. United States Food and Drug Administration (FDA). Guidance for Industry and FDA Staff - Non-Clinical Engineering Tests and Recommended Labeling for Intravascular Stents and Associated Delivery Systems. 2010 [cited April 3, 2012; Available from: http://www.fda.gov/medicaldevices/deviceregulationandguidance/guidancedocuments /ucm071863.htm

41. Machine Solutions, Inc. IDTE 2000 (Intervantional Device Testing Equipment). 2012 [cited April 11, 2012; Available from: http://www.machinesolutions.com/Medical-Device-Performance-Testing/TestingEquipment/Catheter-Guidewire-Testing-Equipment-IDTE2000.htm

42. Laerdal Medical Corporation. Angiogram Sam Arterial Models. 2012 [cited April 11, 2012; Available from: http://angiogramsam.com/arterial-models.html 43. United Biologics, Inc. Angio Suite Training Model. 2012 [cited April 15, 2012; Available from: http://www.unitedbiologics.com/angio-suite-training-model.html

44. Balcon R, Beyar R, Chierchia S, De Scheerder I, Hugenholtz PG, Kiemeneij F, et al. Recommendations on stent manufacture, implantation and utilization. Study Group of the Working Group on Coronary Circulation. Eur Heart J 1997 Oct;18(10):15361547.

45. Kawase Y, Suzuki Y, Ikeno F, Yoneyama R, Hoshino K, Ly HQ, et al. Comparison of nonuniform rotational distortion between mechanical IVUS and OCT using a phantom model. Ultrasound Med Biol 2007 Jan;33(1):67-73. 
46. Finn AV, Gold HK, Tang A, Weber DK, Wight TN, Clermont A, et al. A novel rat model of carotid artery stenting for the understanding of restenosis in metabolic diseases. J Vasc Res 2002 Sep-Oct;39(5):414-425.

47. Langeveld B, Roks AJ, Tio RA, van Boven AJ, van der Want JJ, Henning RH, et al. Rat abdominal aorta stenting: a new and reliable small animal model for in-stent restenosis. J Vasc Res 2004 Sep-Oct;41(5):377-386.

48. Touchard AG, Schwartz RS. Preclinical restenosis models: challenges and successes. Toxicol Pathol 2006;34(1):11-18.

49. Schwartz RS, Edelman ER, Carter A, Chronos N, Rogers C, Robinson KA, et al. Drug-eluting stents in preclinical studies: recommended evaluation from a consensus group. Circulation 2002 Oct 1;106(14):1867-1873.

50. Schwartz RS, Topol EJ, Serruys PW, Sangiorgi G, Holmes DR, Jr. Artery size, neointima, and remodeling: time for some standards. J Am Coll Cardiol 1998 Dec;32(7):2087-2094.

51. Grewe PH, Deneke T, Machraoui A, Barmeyer J, Muller KM. Acute and chronic tissue response to coronary stent implantation: pathologic findings in human specimen. J Am Coll Cardiol 2000 Jan;35(1):157-163.

52. Kipshidze N, Dangas G, Tsapenko M, Moses J, Leon MB, Kutryk M, et al. Role of the endothelium in modulating neointimal formation: vasculoprotective approaches to attenuate restenosis after percutaneous coronary interventions. J Am Coll Cardiol 2004 Aug 18;44(4):733-739.

53. Babapulle MN, Eisenberg MJ. Coated stents for the prevention of restenosis: Part I. Circulation 2002 Nov 19;106(21):2734-2740. 
54. Van Belle E, Tio FO, Couffinhal T, Maillard L, Passeri J, Isner JM. Stent endothelialization. Time course, impact of local catheter delivery, feasibility of recombinant protein administration, and response to cytokine expedition. Circulation 1997 Jan 21;95(2):438-448.

55. Johnson GJ, Griggs TR, Badimon L. The utility of animal models in the preclinical study of interventions to prevent human coronary artery restenosis: analysis and recommendations. On behalf of the Subcommittee on Animal, Cellular and Molecular Models of Thrombosis and Haemostasis of the Scientific and Standardization Committee of the International Society on Thrombosis and Haemostasis. Thromb Haemost 1999 May;81(5):835-843.

56. Mason RG, Read MS. Some Species Differences in Fibrinolysis and Blood Coagulation. Journal of Biomedical Materials Research 1971 Jan;5(1):121-128.

57. Cardinal KO, Bonnema GT, Hofer H, Barton JK, Williams SK. Tissue-Engineered Vascular Grafts as In Vitro Blood Vessel Mimics for the Evaluation of Endothelialization of Intravascular Devices. Tissue Engineering 2006 December 2006;12(12):3431-3438.

58. Cardinal KO, Williams SK. Assessment of the intimal response to a protein-modified stent in a tissue-engineered blood vessel mimic. Tissue Eng Part A 2009 Dec;15(12):3869-3876.

59. Delagrammaticas D. Development of an In Vitro Tissue Engineered Blood Vessel Mimic Using Human Large Vessel Cell Sources. San Luis Obispo: California Polytechnic State University; 2009. 
60. Gyongyosi M, Yang P, Khorsand A, Glogar D. Longitudinal straightening effect of stents is an additional predictor for major adverse cardiac events. Austrian Wiktor Stent Study Group and European Paragon Stent Investigators. J Am Coll Cardiol 2000 May;35(6):1580-1589.

61. Wentzel JJ, Krams R, Schuurbiers JCH, Oomen JA, Kloet J, van der Giessen WJ, et al. Relationship Between Neointimal Thickness and Shear Stress After Wallstent Implantation in Human Coronary Arteries. Circulation 2001;103(13):1740-1745.

62. Wentzel JJ, Whelan DM, van der Giessen WJ, van Beusekom HM, Andhyiswara I, Serruys PW, et al. Coronary stent implantation changes 3-D vessel geometry and 3-D shear stress distribution. J Biomech 2000 Oct;33(10):1287-1295.

63. Duraiswamy N, Schoephoerster RT, Moreno MR, Moore JEJ. Stented Artery Flow Patterns and Their Effects on the Artery Wall. Annual Review of Fluid Mechanics 2007;39:357-382.

64. Bjorkerud S, Bondjers G. Arterial repair and atherosclerosis after mechanical injury. 5. Tissue response after induction of a large superficial transverse injury. Atherosclerosis 1973 Sep-Oct;18(2):235-255.

65. Perez De Prado A, Perez Martinez C, Cuellas Ramon C, Gonzalo Orden JM, Altonaga JR, Iglesias MJ, et al. Endothelialization of nonapposed stent struts located over the origin of a side branch: results with different carbofilm-coated stents. J Interv Cardiol 2009 Jun;22(3):222-227.

66. Shah K. Characterization of Bioreactor Systems for Tissue Engineered Blood Vessels. Industry Advisory Board Poster Session. San Luis Obispo: California Polytechnic State University, 2011. 
67. Suzuki E, Nagata D, Yoshizumi M, Kakoki M, Goto A, Omata M, et al. Reentry into the cell cycle of contact-inhibited vascular endothelial cells by a phosphatase inhibitor. Possible involvement of extracellular signal-regulated kinase and phosphatidylinositol 3-kinase. J Biol Chem 2000 Feb 4;275(5):3637-3644.

68. Grewe PH, Deneke T, Holt SK, Machraoui A, Barmeyer J, Muller KM. [Scanning electron microscopic analysis of vessel wall reactions after coronary stenting]. Z Kardiol 2000 Jan;89(1):21-27.

69. Korossis SA, Bolland F, Kearney JN, Fisher J, Ingham E. Bioreactors in Tissue Engineering. Topics in Tissue Engineering 2005;2:1-23.

70. Martin I, Wendt D, Heberer M. The role of bioreactors in tissue engineering. Trends Biotechnol 2004 Feb;22(2):80-86.

71. Leifer SM. Design and Optimization of a Blood Vessel Mimic Bioreactor System for the Evaluation of Intravascular Devices in Simple and Complex Vessel Geometries. San Luis Obispo: California Polytechnic State University; 2008.

72. Dodge JT, Jr., Brown BG, Bolson EL, Dodge HT. Lumen diameter of normal human coronary arteries. Influence of age, sex, anatomic variation, and left ventricular hypertrophy or dilation. Circulation 1992 Jul;86(1):232-246.

73. Ikeno F, Buchbinder M, Yeung AC. Novel stent and delivery systems for the treatment of bifurcation lesions: porcine coronary artery model. Cardiovasc Revasc Med 2007 Jan-Mar;8(1):38-42.

74. Sprague EA, Luo J, Palmaz JC. Endothelial cell migration onto metal stent surfaces under static and flow conditions. J Long Term Eff Med Implants 2000;10(1-2):97110. 
75. Flaherty JT, Pierce JE, Ferrans VJ, Patel DJ, Tucker WK, Fry DL. Endothelial nuclear patterns in the canine arterial tree with particular reference to hemodynamic events. Circ Res 1972 Jan;30(1):23-33.

76. Kim DW, Langille BL, Wong MK, Gotlieb AI. Patterns of endothelial microfilament distribution in the rabbit aorta in situ. Circ Res 1989 Jan;64(1):21-31.

77. Peña T. Vascular Tissue Engineering and the Advancement of an In Vitro Blood Vessel. San Luis Obispo: California Polytechnic State University; 2009.

78. Inoguchi H, Tanaka T, Maehara Y, Matsuda T. The effect of gradually graded shear stress on the morphological integrity of a HUVEC-seeded compliant small-diameter bascular graft. Biomaterials 2007;28:486-495.

79. Ott MJ, Ballermann BJ. Shear stress-conditioned, endothelial cell-seeded vascular grafts: improved cell adherence in response to in vitro shear stress. Surgery 1995 Mar;117(3):334-339.

80. Sank A, Rostami K, Weaver F, Ertl D, Yellin A, Nimni M, et al. New evidence and new hope concerning endothelial seeding of vascular grafts. Am J Surg 1992 Sep;164(3):199-204.

81. De Scheerder IK, Wilczek KL, Verbeken EV, Vandorpe J, Lan PN, Schacht E, et al. Biocompatibility of polymer-coated oversized metallic stents implanted in normal porcine coronary arteries. Atherosclerosis 1995 Apr 7;114(1):105-114.

82. Rogers C, Tseng DY, Squire JC, Edelman ER. Balloon-artery interactions during stent placement: a finite element analysis approach to pressure, compliance, and stent design as contributors to vascular injury. Circ Res 1999 Mar 5;84(4):378-383. 
83. Ur S. HUVEC and HUVSMC on PLGA. Cal Poly BVM Laboratory Meeting. San Luis Obispo: California Polytechnic State University, 2011.

84. Smith A. Characterization and Implementation of a Decellularized Porcine Vessel as a Biologic Scaffold for a Blood Vessel Mimic, Appendix B.16. San Luis Obispo: California Polytechnic State University; 2011. 


\section{Appendix A: Abbreviations}

ACC - American College of Cardiology

AHA - American Heart Association

ANOVA - Analysis of variance

BBI - Bisbenzimide

BMS - Bare metal stent

BVM - Blood vessel mimic

CHD - Coronary heart disease

DDES - Dual drug-eluting stent

DES - Drug-eluting stent

$\mathrm{D}_{1}-$ Distal 1

$\mathrm{D}_{2}-$ Distal 2

ePTFE - expanded polytetrafluoroethylene

ESS - Endothelial shear stress

EtO - Ethylene oxide

FBS - Fetal bovine serum

FDA - Food and Drug Administration

GA-1000 - gentamicin sulfate amphotericin B

hEGF - human epidermal growth factor

HEPES - 4-(2-hydroxyethyl)-1-piperazineethanesulfonic acid

hFGF-B - human fibroblast growth factor B

HUVEC - Human umbilical vein endothelial cell

HUVSMC - Human umbilical vein smooth muscle cell

ID - Inner diameter

IDTE2000 - Interventional Device Testing Equipment

IVUS - Intravascular ultrasound

M - Middle

NIRS - Near infrared spectroscopy

OD - Outer diameter

$\mathrm{P}_{1}$ - Proximal 1

$\mathrm{P}_{2}-$ Proximal 2

$\mathrm{P} / \mathrm{S}$ - penicillin/streptomycin

PTCA - Percutaneous transluminal coronary angioplasty

PTFE - polytetrafluoroethylene

R3-IGF-1 - long R insulin-like growth factor 1

SEM - Scanning electron microscopy

TCFA - thin-cap fibroatheroma

VEGF - vascular endothelial growth factor 


\section{Appendix B: Protocol for Maintaining Multifunctional Bioreactor}

\section{Purpose}

The O-rings of multifunctional bioreactors may degrade and cause leakage during bioreactor use, which could ruin an experiment. Therefore, it is important to replace Orings on multifunctional bioreactors when the O-rings seem to have started to degrade. The following protocol describes how to replace O-rings on multifunctional bioreactors. A similar protocol could be used to possibly repair leaky two-port lids.

\section{Components}

- Razor blade (rib-back carbon steel, Bard-Parker)

- Hemostats

- New O-rings

\section{Picture-Protocol}

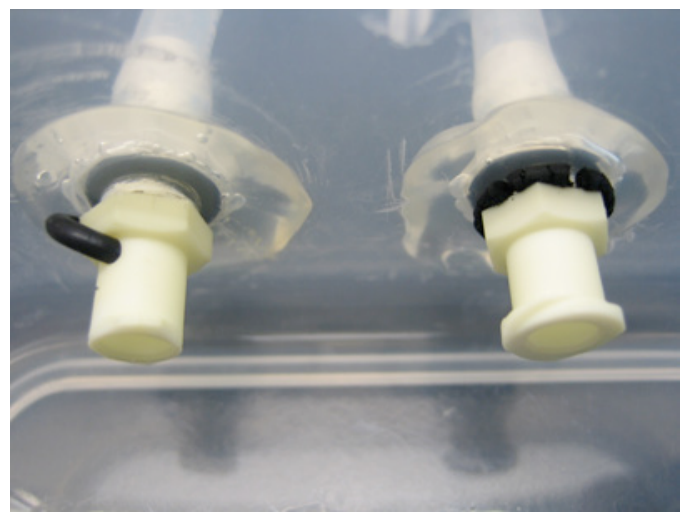

Figure B.1. Locate degraded O-rings.
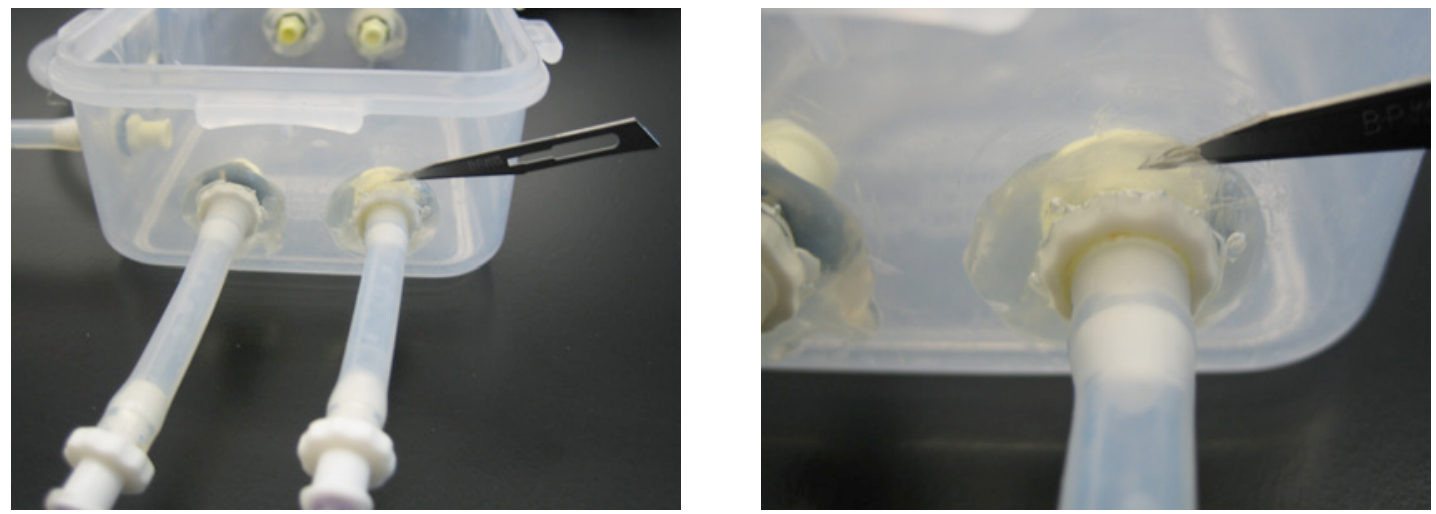

Figure B.2. Wedge razor blade between UV glue and polypropylene container, and start to pry off UV glue. 

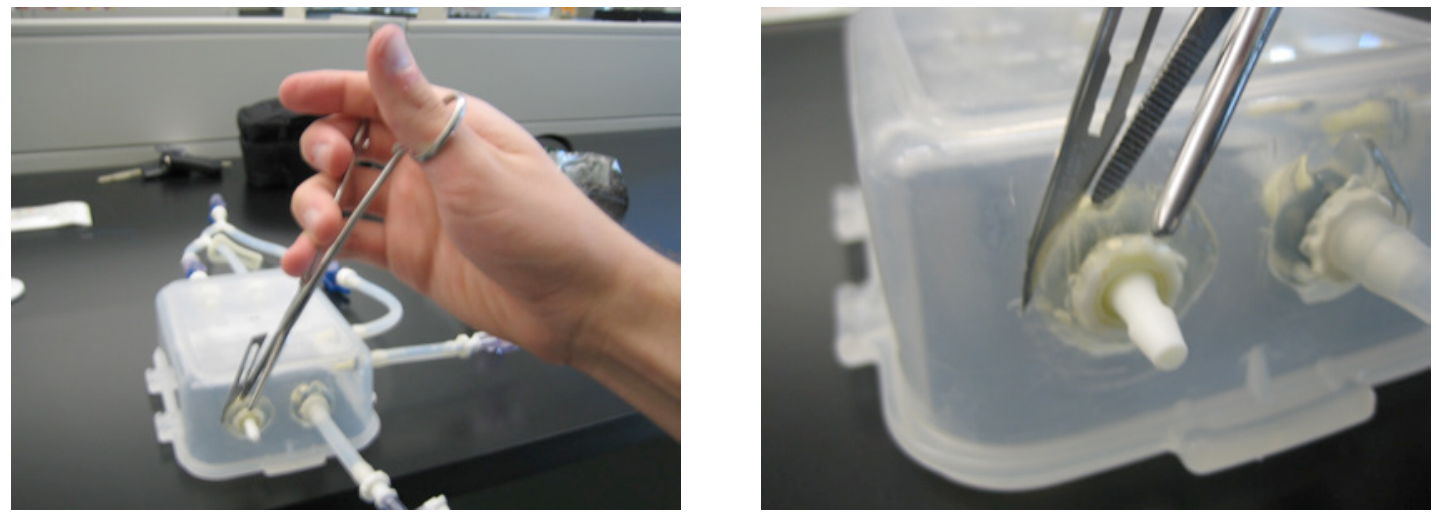

Figure B.3. Leave razor blade wedged in place, and wedge hemostats between UV glue and polypropylene container.
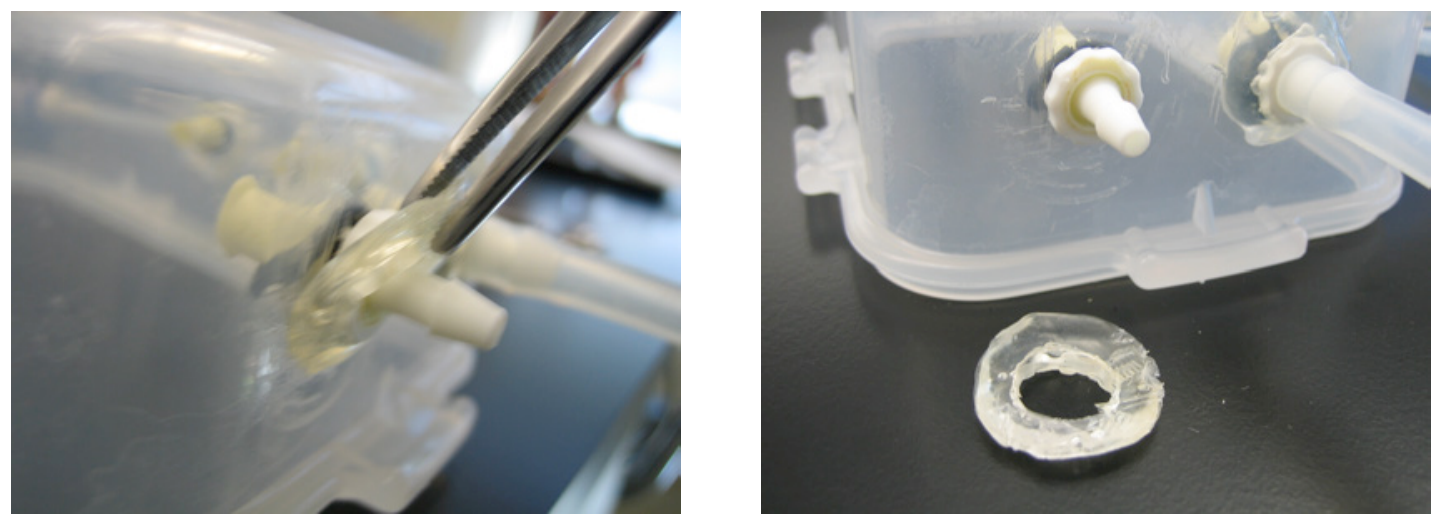

Figure B.4. Remove razor blade. Use hemostats to pry off UV glue from polypropylene container. Use hemostats to unscrew lock nut and to start unscrewing femal luer lug panel mount. Finish unscrewing female luer lug panel mount by hand. Replace O-rings. Screw female luer lug panel mount back in place. Screw lock nut back in place. Re-apply UV glue. 


\section{Appendix C: HUVEC Media Preparation}

\section{Purpose}

The following was the list of components used to make HUVEC media and the protocol that was followed to make this media. This protocol yielded a single bottle of $515.2 \mathrm{~mL}$ of HUVEC media. The HUVEC media consisted of $500 \mathrm{~mL}$ of endothelial cell basal medium and EGM ${ }^{\mathrm{TM}}-2$ SingleQuots ${ }^{\circledR}$ made by Lonza with catalog number CC-4176.

\section{Components}

- $500 \mathrm{~mL}$ endothelial cell basal medium

- $10 \mathrm{~mL}$ fetal bovine serum (FBS)

- $0.2 \mathrm{~mL}$ hydrocortisone

- $2 \mathrm{~mL}$ human fibroblast growth factor B (hFGF-B)

- $0.5 \mathrm{~mL}$ vascular endothelial growth factor (VEGF)

- $0.5 \mathrm{~mL}$ long $\mathrm{R}$ insulin-like growth factor 1 (R3-IGF-1)

- $0.5 \mathrm{~mL}$ ascorbic acid

- $0.5 \mathrm{~mL}$ human epidermal growth factor (hEGF)

- $0.5 \mathrm{~mL}$ gentamicin sulfate amphotericin B (GA-1000)

- $0.5 \mathrm{~mL}$ heparin

\section{Protocol}

1. In a laminar flow hood, attach filter to bottle.

2. Attach vacuum to filter. Turn on vacuum.

3. Pour approximately $400 \mathrm{~mL}$ of endothelial cell basal medium into filter, leaving the remaining $100 \mathrm{~mL}$ to wash down other components from sides of filter.

4. Add the remaining components into the filter, using a pipette to verify the exact quantities of each component.

5. Wash the filter with the remaining $100 \mathrm{~mL}$ of endothelial cell basal medium.

6. Detach the filter from the bottle. Cap the bottle. Place Lonza label on bottle, and place tape label on bottle that includes date and initials.

7. Place labeled bottle in refrigerator $\left(4^{\circ} \mathrm{C}\right)$.

8. Before using media from refrigerator, warm media to $37^{\circ} \mathrm{C}$ in water bath. 


\section{Appendix D: Bioreactor Media Preparation}

\section{Purpose}

The following was the list of components used to make bioreactor media and the protocol that was followed to make this media. This protocol yielded a single bottle of $569.4 \mathrm{~mL}$ of bioreactor media.

\section{Components}

- $500 \mathrm{~mL}$ M199

- $56 \mathrm{~mL}$ fetal bovine serum (FBS)

- $5 \mathrm{~mL}$ L-glutamine

- $5 \mathrm{~mL}$ penicillin/streptomycin solution $(\mathrm{P} / \mathrm{S})$

- $0.6 \mathrm{~mL}$ fungizone

- $2.8 \mathrm{~mL}$ 4-(2-hydroxyethyl)-1-piperazineethanesulfonic acid (HEPES)

\section{Protocol}

1. In a laminar flow hood, attach filter to bottle.

2. Attach vacuum to filter. Turn on vacuum.

3. Pour approximately $400 \mathrm{~mL}$ of M199 into filter, leaving the remaining $100 \mathrm{~mL}$ to wash down other components from sides of filter.

4. Add the remaining components into the filter, using a pipette to verify the exact quantities of each component.

5. Wash the filter with the remaining $100 \mathrm{~mL}$ of M199.

6. Detach the filter from the bottle. Cap the bottle. Place tape label on bottle that includes "Bioreactor Media," date, and initials.

7. Place labeled bottle in refrigerator $\left(4^{\circ} \mathrm{C}\right)$.

8. Before using media from refrigerator, warm media to $37^{\circ} \mathrm{C}$ in water bath. 


\section{Appendix E: Conditioning Media Preparation}

\section{Purpose}

The following was the list of components used to make conditioning media and the protocol that was followed to make this media. This protocol yielded a single bottle of $588.6 \mathrm{~mL}$ of conditioning media.

\section{Components}

- $500 \mathrm{~mL}$ M199

- $83 \mathrm{~mL}$ fetal bovine serum (FBS)

- $5 \mathrm{~mL}$ penicillin/streptomycin solution $(\mathrm{P} / \mathrm{S})$

- $0.6 \mathrm{~mL}$ fungizone

\section{Protocol}

1. In a laminar flow hood, attach filter to bottle.

2. Attach vacuum to filter. Turn on vacuum.

3. Pour approximately $400 \mathrm{~mL}$ of M199 into filter, leaving the remaining $100 \mathrm{~mL}$ to wash down other components from sides of filter.

4. Add the remaining components into the filter, using a pipette to acquire the exact quantities of each component.

5. Wash the filter with the remaining $100 \mathrm{~mL}$ of M199.

6. Detach the filter from the bottle. Cap the bottle. Place tape label on bottle that includes "Conditioning Media," date, and initials.

7. Place labeled bottle in refrigerator $\left(4^{\circ} \mathrm{C}\right)$.

8. Before using media from refrigerator, warm media to $37^{\circ} \mathrm{C}$ in water bath. 


\section{Appendix F: Complex-Shaped BVM Harvesting Protocol}

\section{Purpose}

The following was the protocol used to remove complex-shaped BVM systems from the large incubator and pump and to harvest complex-shaped BVMs from multifunctional bioreactors.

\section{Components}

- Razor blade (rib-back carbon steel, Bard-Parker)

- Forceps

- $8 \mathrm{~mL}$ of Histochoice per complex-shaped BVM

- Complex-shaped BVM system, including complex-shaped BVM

\section{Protocol}

1. Put on gloves.

2. Close all stopcocks on bioreactor, and clamp off inlet and outlet on reservoir.

3. Detach the complex-shaped BVM system from pump, and move complex-shaped BVM system out of large incubator and onto nonsterile surface.

4. Acquire $15-\mathrm{mL}$ conical for each BVM, and label each $15-\mathrm{mL}$ conical with all information necessary to identify the BVM that will be placed in conical and to identify the orientation of the BVM after placement in conical.

5. Fill each conical with $8 \mathrm{~mL}$ of Histochoice. Leave conicals uncapped.

6. Remove bioreactor lid.

7. Place razor blade at edge of BVM, and cut entirely through BVM, making sure to not do anything that could disrupt the cell lining of BVM. Cut other end of BVM.

8. Use forceps to gently grab end of BVM, and transfer to conical. Place lid on conical.

9. Remove ePTFE scraps that remain on female luer thread barbed fittings.

10. Dispose of all media in bioreactor and reservoir.

11. Clean all bioreactors, bioreactor lids, two-port lids, and tubing with hot water. Dry and store equipment for future experiments. 


\section{Appendix G: BBI Imaging Protocol}

\section{Purpose}

The following was the protocol used for staining complex-shaped BVMs with BBI and for acquiring fluorescent en face BBI images using an Olympus BX41 microscope. The BBI stain that was used Hoechst 33258 pentahydrate by Invitrogen.

\section{Components}

- $10 \mu \mathrm{L}$ BBI stock solution

- $10 \mathrm{~mL}$ milli-Q water

- Forceps

- Foil

- $\quad$ 15-mL conicals for each sample

\section{Protocol}

1. Wrap nonsterile 15-mL conicals in foil. Thoroughly label conicals for all samples.

2. Make BBI solution. Always keep stock solution wrapped in foil!

a. Use small tube of stock solution and dilute 1:1000 with milli-Q water $(10 \mu \mathrm{L}$ stock solution in $10 \mathrm{~mL}$ water). Use pipette aid from room 209 hood for milli-Q water; spray pipette aid before placing back in hood.

b. Mix by inverting.

3. Put on safety glasses. Cut samples with blade or scissors, being careful to not disrupt cell lining inside lumen (squeezing, touching, and scraping can all disrupt lining and ruin experiment).

4. Use washed forceps to place samples in corresponding $15-\mathrm{mL}$ conicals that contain BBI solution. Carefully keep track of samples. Leave foil on conicals.

a. Let samples soak for at least 15 minutes (longer is better).

5. Put away extra stock solution. Clean up preparation area.

6. With permission or help from Dr. Cardinal, use fluorescent microscope to obtain en face images. Take forceps to use at microscope. See figure G. 1 for pictures of steps below.

a. Log into notebook (fluorescence; initials; date; time and lamp hours).

b. Turn turret to setting 4 .

c. Turn on Olympus lamp (green switch).

d. Turn on Optiscan wheels (black switch).

e. Turn on camera (black switch).

f. Set filter wheel 1 to 1 , and set filter wheel 2 to 1 .

g. Open shutter: o

h. Set Prior keypad to shutter S1.

i. Dial objectives to desired magnification (typically $4 x$ and 10x for BBI images).

j. Set thin bar to icon of eye and camera.

k. Sign into computer by clicking Dr. Cardinal's account (password can be obtained from Dr. Cardinal). 
1. Click QCapture Pro (on desktop).

m. Click camera icon at top of QCapture Pro window: 国

n. Place slide on microscope. Place sample on slide.

o. See figure G.2.

p. Manually adjust microscope to clarify image.

q. If scaffold is too wet, carefully blot end of scaffold on Kim wipe.

r. Take pictures. Save pictures if desired (labeled with sample info, initials, and magnification).

s. Quit QCapture Pro. Log out of computer.

t. Shut down microscope by switching off Olympus lamp, switching off Optiscan wheels, switching off camera (on top of microscope), and closing shutter.

u. Log out of notebook.

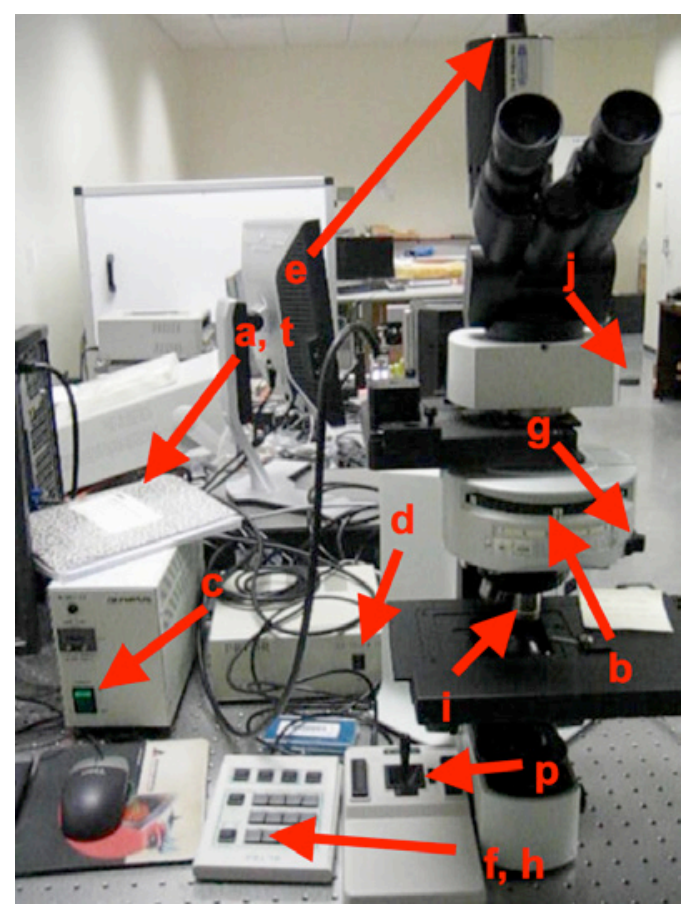

Figure G.1. Microscope anatomy. Letters correspond to step 6 in the protocol. 


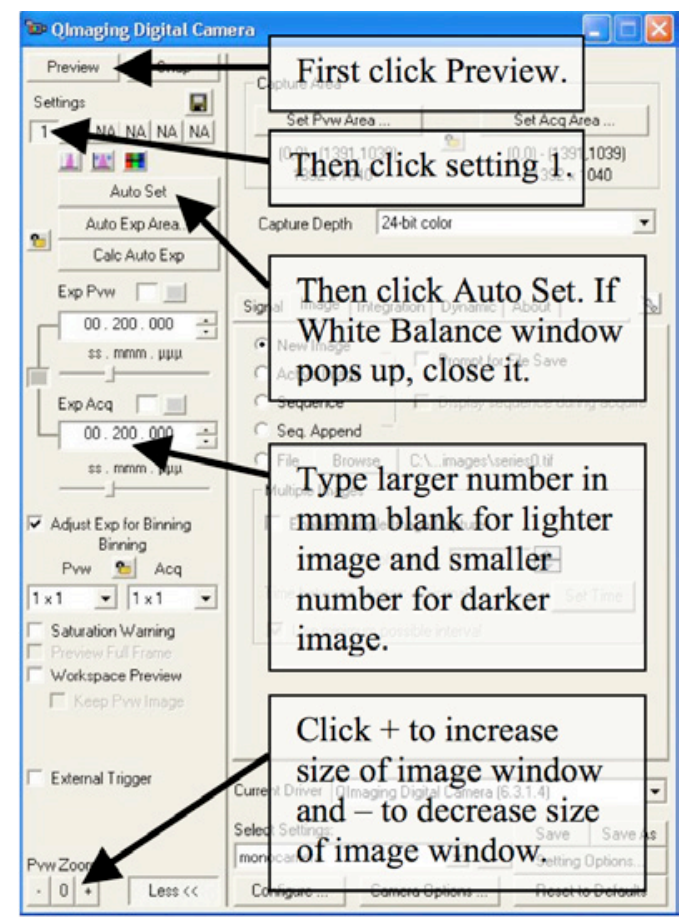

Figure G.2. QCapture Pro control panel. 


\section{Appendix H: Protocol for Developing HUVEC-Sodded Complex-Shaped BVMs}

\section{Purpose}

The following protocol was used to tissue-engineer HUVEC-only S-, L-, and U-shaped BVMs. This protocol has not been used to tissue-engineer bifurcated BVMs.

\section{Components}

- $\quad$ ePTFE scaffold(s)

- 1.5 million HUVECs $/ \mathrm{cm}^{2}$ of scaffold

- Conditioning media (See Appendix E)

- Bioreactor media (See Appendix D)

- HUVEC media (See Appendix C)

- Sterile $70 \%$ EtOH and sterile $100 \% \mathrm{EtOH}$

- Silk Suture

- Forceps

- Degassing flask with rubber stopper

- Multifunctional bioreactor (including 3 luer plugs and 2 rotating male-to-male luer lock connectors)

- Peristaltic pump

- Sterile gloves

\section{Protocol}

Preparation (Approximately 1.5 weeks prior to "Setup")

1. Wash all multifunctional bioreactors, bioreactor lids, and two-port lids with water, and dry overnight.

2. Sterilize multifunctional bioreactors, bioreactor lids, and two-port lids by placing them in individual sterilization bags and taking them to the Cal Poly Vet Clinic for EtO gassing.

3. Thaw, plan out calendar of cell culture, and begin cell culture.

4. Cut ePTFE scaffolds to the dimensions described in Section 2.3.2, and wear gloves while doing so.

Preparation (Approximately 1 day prior to "Setup")

5. Sterilize ePTFE scaffolds, forceps, and degassing flask

6. Make media (listed in components list above).

7. Degass conditioning media

8. Denucleate scaffolds by placing first placing them in $70 \% \mathrm{EtOH}$ for 10 minutes and then $100 \% \mathrm{EtOH}$ for 10 minutes.

9. Protein-coat scaffolds by placing them in the degassed conditioning media overnight in the small incubator at $37^{\circ} \mathrm{C}$.

"Setup" Day

10. Warm up bioreactor media, conditioning media, and HUVEC media in water bath. 
11. Fill biochamber with bioreactor media.

12. Insert sterile graft into biochamber using sterile gloves.

13. Number biochamber lids with tape and marker.

14. Using syringe, flush lumen with conditioning media to prime scaffold.

15. Clamp lumen and continue to prime scaffold.

16. Repeat for all scaffolds.

17. Prime 2-port reservoirs with conditioning media.

18. Attach primed biochamber to 2-port reservoir and condition scaffold for 10 minutes. Administer flow through lumen first to remove air, then clamp lumen and condition transmurally on 150-rpm setting.

19. Leave primed biochambers in large incubator until ready for sodding step.

20. Prime 2-port reservoirs with HUVEC. Prepare a reservoir for each vessel. Be sure that drip is visible and outlet is submerged. Clamp tubing and leave in big incubator.

21. Take primed biochambers and reservoirs to hood.

22. Attach outlet of reservoir to inlet stopcock of biochamber.

23. Leave reservoir inlet unattached and biochamber outlet facing trough.

24. Harvest HUVECs from T225: apply trypsin; deactivate with HUVEC media.

25. Pellet cell suspension (on centrifuge setting 4 for 4 minutes).

26. Resuspend pellet in $4 \mathrm{~mL}$ HUVEC media.

27. Prepare one trough of $10 \mathrm{~mL}$ HUVEC media and one trough of $4 \mathrm{~mL}$ cell suspension.

28. Prime tubing by capping off proximal stopcock so fluid will flow back into reservoir. Inject $5 \mathrm{~mL}$ HUVEC media. Immediately replace stopcock to 45-degree angle. Detach syringe.

29. Sod cells.

a. Take up $4 \mathrm{~mL}$ cell solution in syringe.

b. Slightly twist stopcock so fluid bulges out of tip of stopcock.

c. Attach syringe to stopcock.

d. Toggle stopcock to allow fluid to flow only distally through graft.

e. Inject $4 \mathrm{~mL}$ cell solution.

f. While still pressuring syringe, cap off stopcock to 45-degree angle. Detach syringe.

30. Chase cells by repeating step 29 with $4 \mathrm{~mL}$ HUVEC media instead of $4 \mathrm{~mL}$ cell solution.

31. Replace old reservoir with new HUVEC media reservoir. Tighten reservoir cap.

32. Move complex-shaped BVM system to large incubator.

33. Maintain transmural flow at $10 \mathrm{rpm}$ for 30 minutes.

34. Stop transmural flow, and maintain luminal flow at $15 \mathrm{rpm}$ for 24 hours.

35 . Repeat steps 22 through 34 for each bioreactor.

Day After "Setup Day"

36. Take down 1-day BVMs (Appendix F).

37. Perform desired adjustment of flow rate depending on the experiment being performed.

38. Take down BVMs at correct time points. 


\section{Appendix I: Raw Data for Cell Deposition Study}

\section{Purpose}

This appendix includes BBI images from each region along the length of the 6 complexshaped BVMs of Chapter 3 (Figures I.1-I.6), raw cell counts for L- and U-shaped BVMs (Tables I.1-I.2 respectively), and a description of the method used to convert raw cell counts to cell densities with units of cells $/ \mathrm{cm}^{2}$.

\section{Figures}

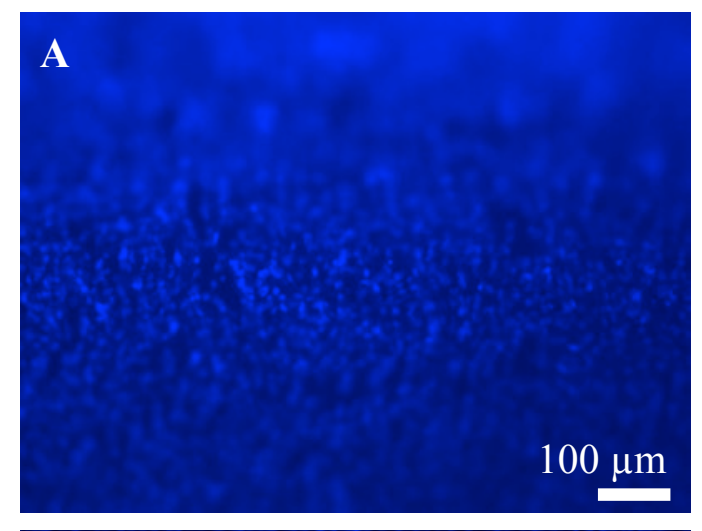

\section{B}

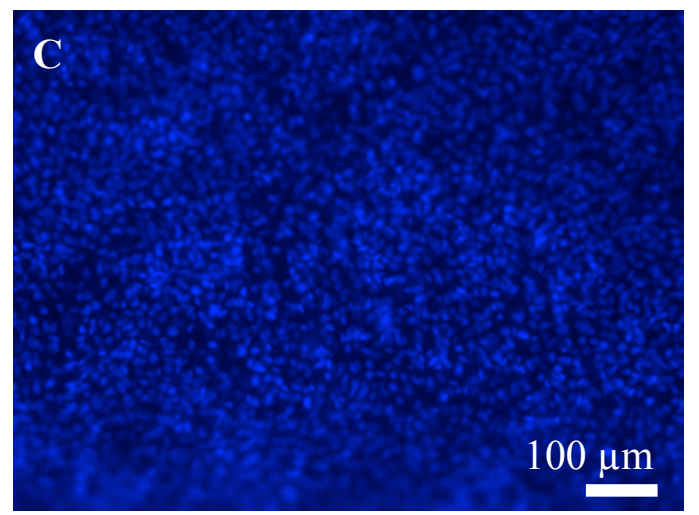

\section{D}

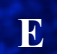

$100 \mu \mathrm{m}$

Figure I.1. L-Shaped BVM 1. A BBI image is shown for each segment along the length of the BVM: Proximal 1 (A), Proximal 2 (B), Middle (C), Distal 1 (D), and Distal 2 (E). 


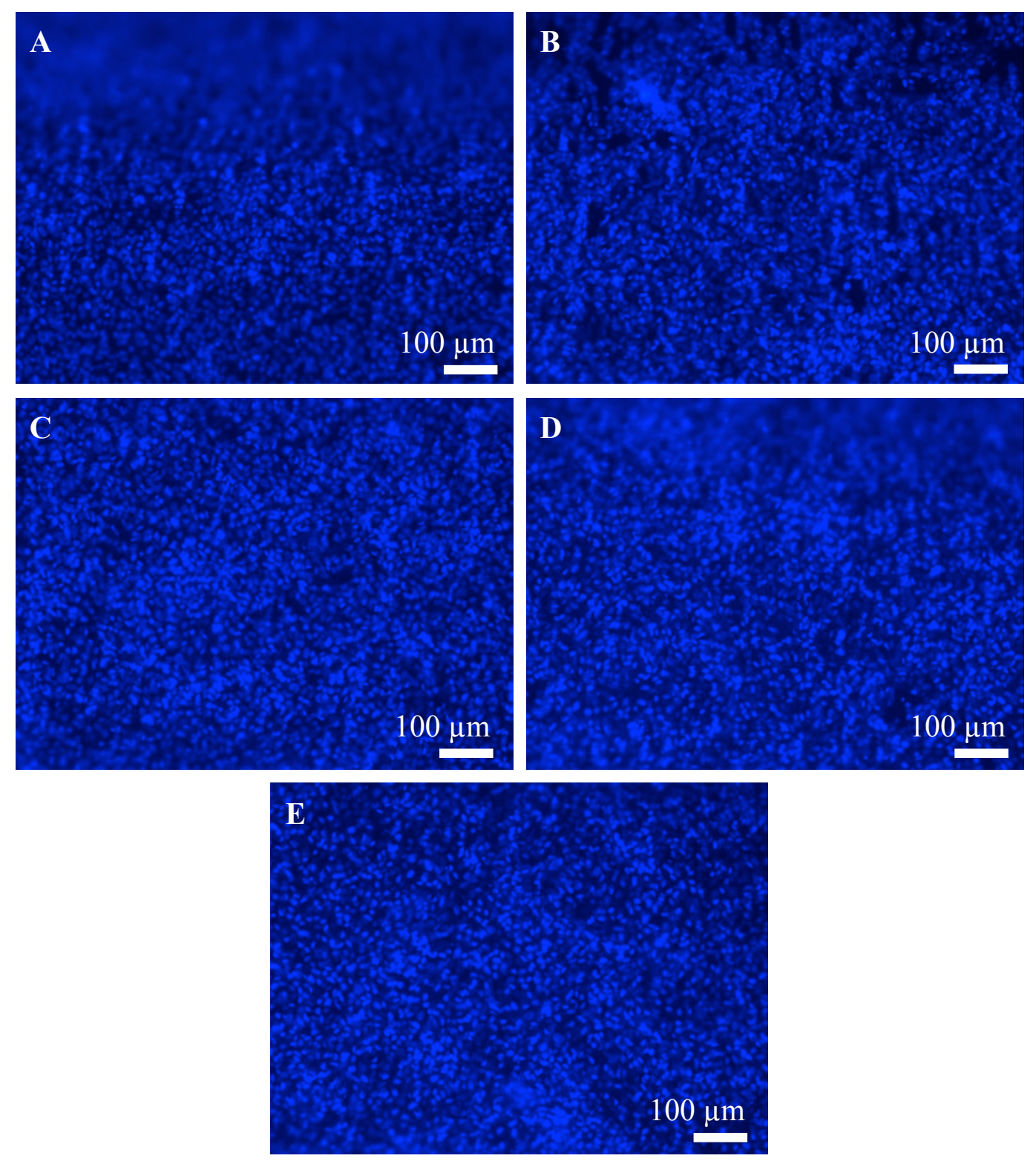

Figure I.2. L-Shaped BVM 2. A BBI image is shown for each segment along the length of the BVM: Proximal 1 (A), Proximal 2 (B), Middle (C), Distal 1 (D), and Distal 2 (E). 


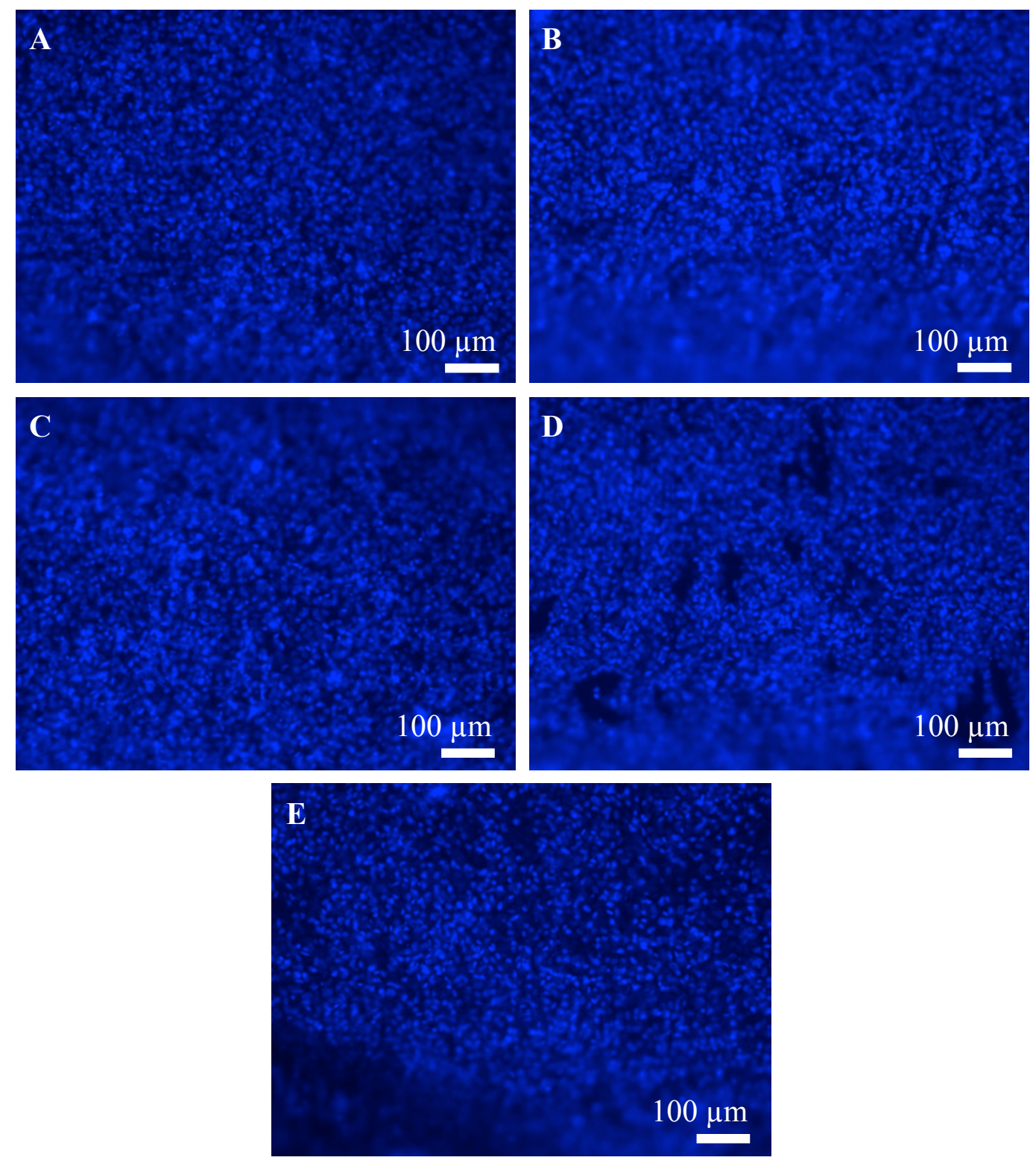

Figure I.3. L-Shaped BVM 3. A BBI image is shown for each segment along the length of the BVM: Proximal 1 (A), Proximal 2 (B), Middle (C), Distal 1 (D), and Distal 2 (E). 


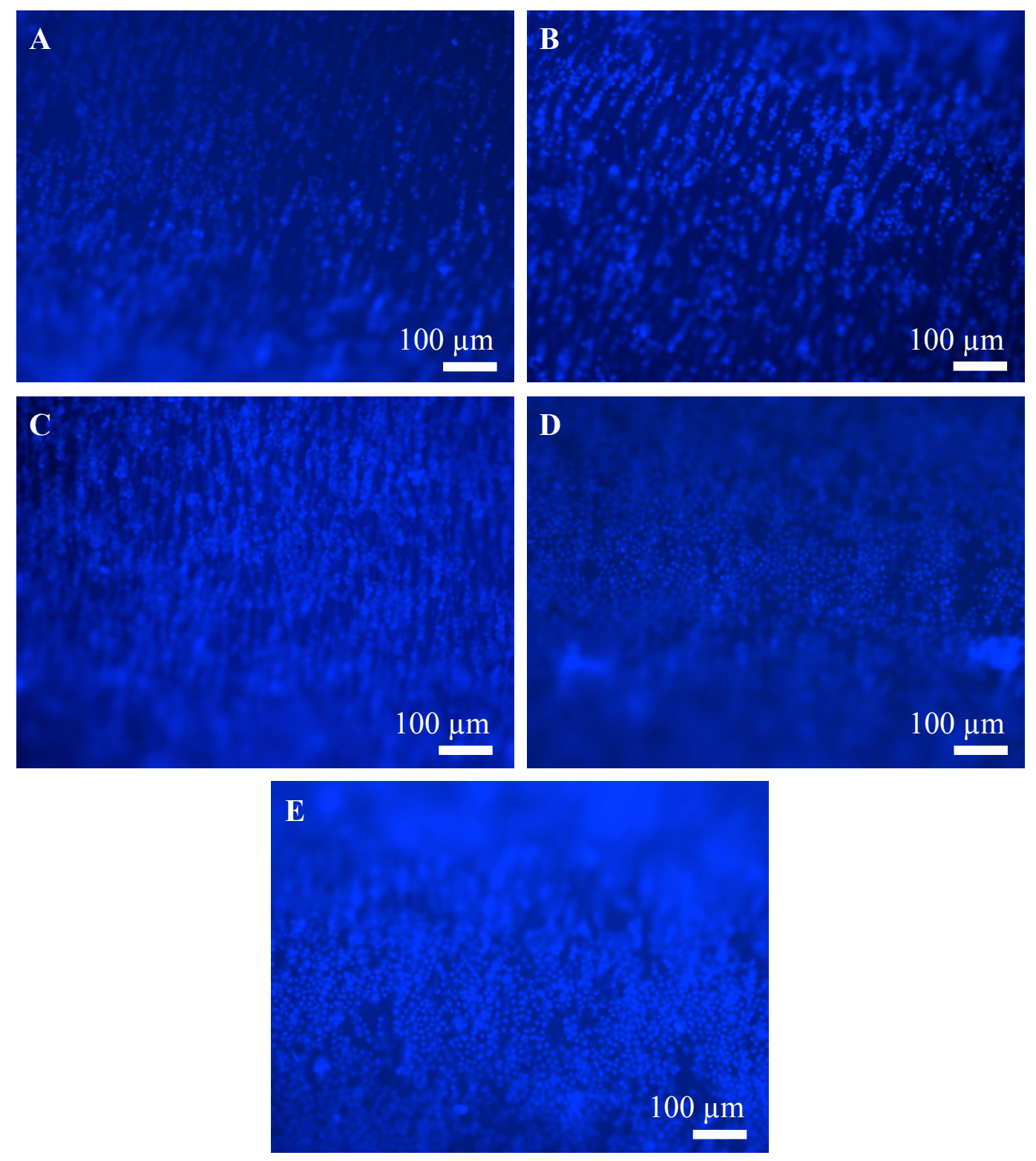

Figure I.4. U-Shaped BVM 1. A BBI image is shown for each segment along the length of the BVM: Proximal 1 (A), Proximal 2 (B), Middle (C), Distal 1 (D), and Distal 2 (E). 

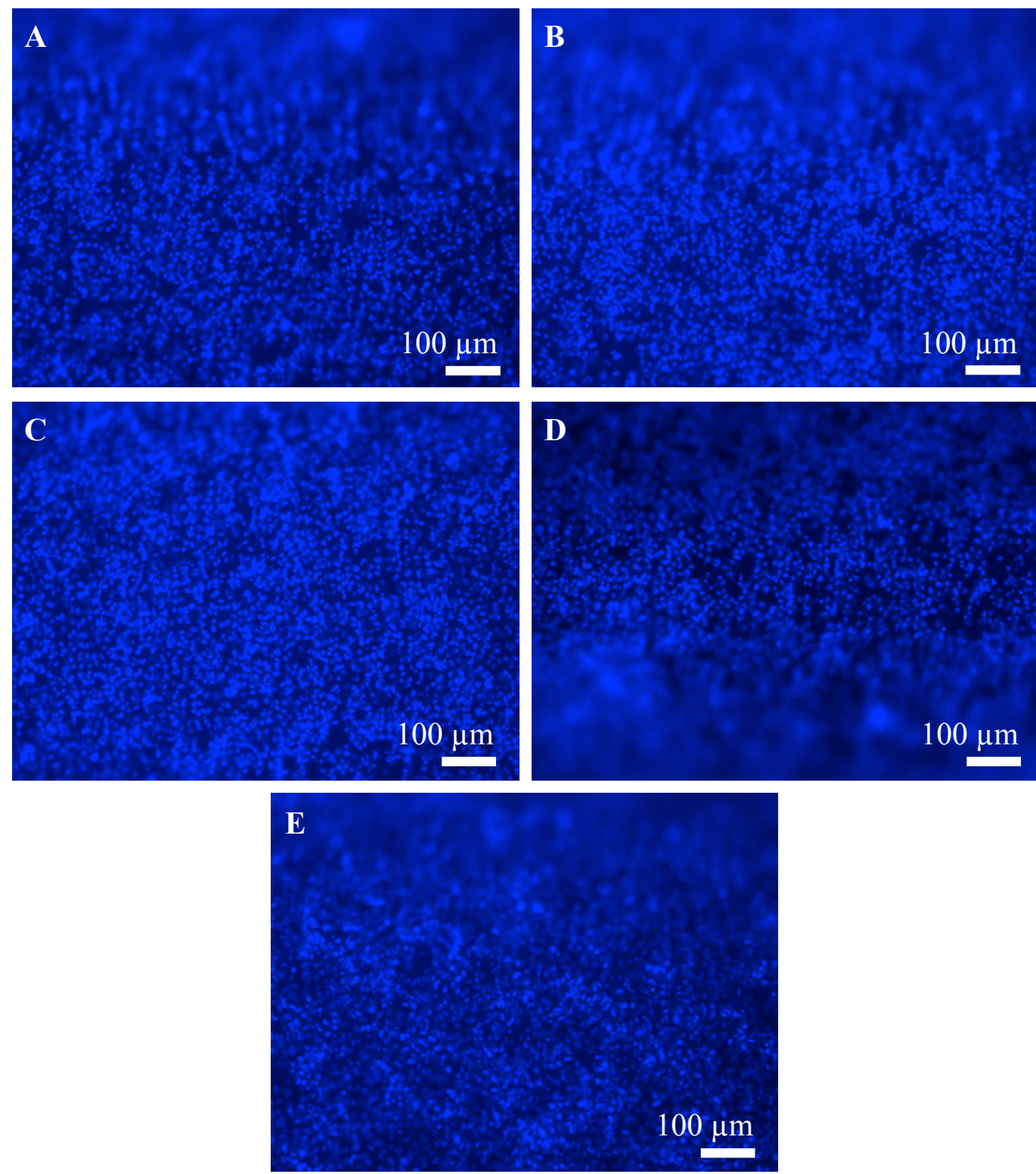

Figure I.5. U-Shaped BVM 2. A BBI image is shown for each segment along the length of the BVM: Proximal 1 (A), Proximal 2 (B), Middle (C), Distal 1 (D), and Distal 2 (E). 

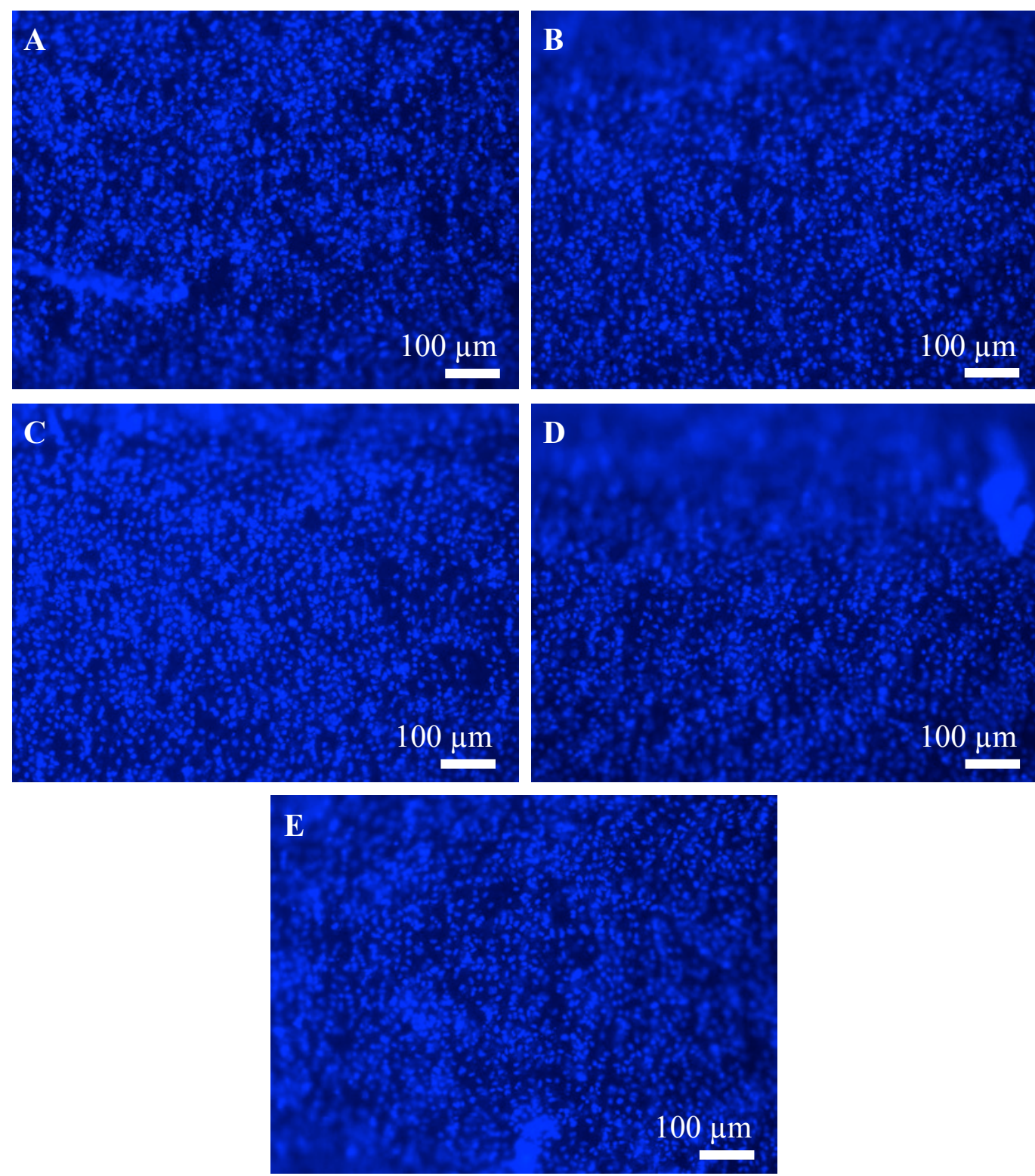

Figure I.6. U-Shaped BVM 3. A BBI image is shown for each segment along the length of the BVM: Proximal 1 (A), Proximal 2 (B), Middle (C), Distal 1 (D), and Distal 2 (E). 
Tables

Table I.1. Raw Cell Counts for 3 L-Shaped BVMs

\begin{tabular}{|c|c|c|c|c|c|c|c|c|c|c|}
\hline BVM & \multicolumn{2}{|c|}{ Distal 2} & \multicolumn{2}{|c|}{ Distal 1} & \multicolumn{2}{|c|}{ Middle } & \multicolumn{2}{|c|}{ Proximal 2} & \multicolumn{2}{|c|}{ Proximal 1} \\
\hline \multicolumn{11}{|l|}{ 1: Inner } \\
\hline Top & 30 & 28 & 30 & 30 & 30 & 28 & 29 & 35 & 31 & 30 \\
\hline Bottom & 29 & 30 & 33 & 32 & 29 & 27 & 29 & 26 & 30 & 30 \\
\hline \multicolumn{11}{|l|}{ 1: Outer } \\
\hline Top & 32 & 31 & 30 & 31 & 32 & 30 & 31 & 30 & 28 & 29 \\
\hline Bottom & 37 & 35 & 32 & 33 & 32 & 30 & 29 & 32 & 30 & 28 \\
\hline \multicolumn{11}{|l|}{ 2: Inner } \\
\hline Top & 34 & 30 & 32 & 32 & 33 & 31 & 31 & 31 & 29 & 29 \\
\hline Bottom & 31 & 31 & 32 & 31 & 33 & 31 & 31 & 31 & 29 & 31 \\
\hline \multicolumn{11}{|l|}{ 2: Outer } \\
\hline Top & 29 & 32 & 31 & 35 & 34 & 34 & 33 & 33 & 32 & 30 \\
\hline Bottom & 28 & 29 & 35 & 31 & 33 & 37 & 33 & 34 & 30 & 33 \\
\hline \multicolumn{11}{|l|}{ 3: Inner } \\
\hline Top & 29 & 32 & 33 & 36 & 35 & 37 & 33 & 31 & 30 & 29 \\
\hline Bottom & 27 & 33 & 32 & 32 & 36 & 36 & 33 & 32 & 30 & 28 \\
\hline \multicolumn{11}{|l|}{ 3: Outer } \\
\hline Top & 27 & 31 & 33 & 33 & 36 & 36 & 34 & 35 & 28 & 33 \\
\hline Bottom & 28 & 31 & 40 & 36 & 35 & 39 & 35 & 33 & 33 & 34 \\
\hline
\end{tabular}


Table I.2. Raw Cell Counts for 3 U-Shaped BVMs

\begin{tabular}{|c|c|c|c|c|c|c|c|c|c|c|}
\hline BVM & \multicolumn{2}{|c|}{ Distal 2} & \multicolumn{2}{|c|}{ Distal 1} & \multicolumn{2}{|c|}{ Middle } & \multicolumn{2}{|c|}{ Proximal 2} & \multicolumn{2}{|c|}{ Proximal 1} \\
\hline \multicolumn{11}{|l|}{ 1: Inner } \\
\hline Top & 31 & 32 & 34 & 30 & 24 & 24 & 25 & 30 & 29 & 26 \\
\hline Bottom & 31 & 35 & 35 & 24 & 32 & 27 & 30 & 33 & 22 & 24 \\
\hline \multicolumn{11}{|c|}{ 1: Outer (Cells were not counted in the outer half of U-shaped BVM 1) } \\
\hline \multicolumn{11}{|l|}{ Top } \\
\hline \multicolumn{11}{|l|}{ Bottom } \\
\hline \multicolumn{11}{|l|}{ 2: Inner } \\
\hline Top & 25 & 22 & 22 & 23 & 28 & 27 & 24 & 28 & 25 & 27 \\
\hline Bottom & 23 & 22 & 27 & 31 & 31 & 31 & 30 & 31 & 32 & 27 \\
\hline \multicolumn{11}{|l|}{ 2: Outer } \\
\hline Top & 30 & 29 & 30 & 35 & 32 & 31 & 29 & 28 & 21 & 23 \\
\hline Bottom & 26 & 29 & 30 & 28 & 27 & 31 & 27 & 26 & 19 & 20 \\
\hline \multicolumn{11}{|l|}{ 3: Inner } \\
\hline Top & 25 & 24 & 29 & 29 & 30 & 28 & 32 & 26 & 23 & 26 \\
\hline Bottom & 26 & 28 & 28 & 27 & 29 & 30 & 28 & 26 & 27 & 23 \\
\hline \multicolumn{11}{|l|}{ 3: Outer } \\
\hline Top & 29 & 25 & 32 & 34 & 30 & 35 & 32 & 32 & 37 & 33 \\
\hline Bottom & 23 & 28 & 31 & 32 & 33 & 29 & 28 & 31 & 29 & 29 \\
\hline
\end{tabular}

\section{Method for Converting Cell Counts to Cell Densities}

Each side of the small white boxes in Figure 3.7 is $100 \mu \mathrm{m}$, therefore each small white box has an area of $10,000 \mu \mathrm{m}^{2}$. To convert cell counts to cell densities, the average cell count for each image was divided by the area of one small white box and then multiplied by the following:

$$
\left(\frac{1,000,000 \mu \mathrm{m}}{1 \mathrm{~m}}\right)^{2}\left(\frac{1 \mathrm{~m}}{100 \mathrm{~cm}}\right)^{2}
$$




\section{Appendix J: Minitab Output for Cell Deposition Study}

\section{Purpose}

This appendix contains the Minitab output that was generated for the statistical analyses of L- and U-shaped BVMs in Chapter 3 (Figures J.1-J.2).

\section{Minitab Output}

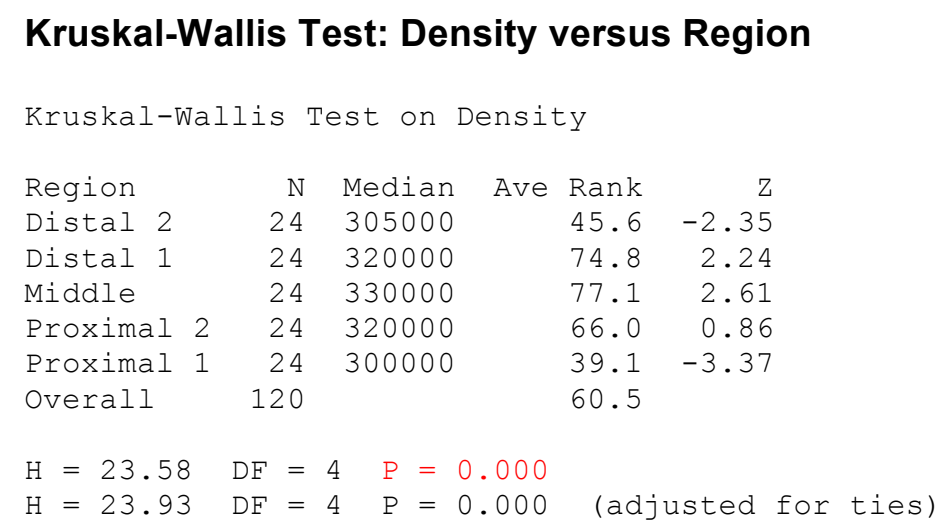

Kruskal-Wallis Test: Density versus Inner/Outer

$\begin{array}{lrrrr}\text { Kruskal-Wallis } & \text { Test on Density } \\ \begin{array}{lrlrl}\text { Inner/Outer } & N & \text { Median } & \text { Ave Rank } & \mathrm{Z} \\ \text { Inner } & 60 & 310000 & 52.5 & -2.53 \\ \text { Outer } & 60 & 320000 & 68.5 & 2.53 \\ \text { Overall } & 120 & & 60.5 & \\ & & & & \\ \mathrm{H}=6.39 & \mathrm{DF}=1 & \mathrm{P}=0.011 & \\ \mathrm{H}=6.48 & \mathrm{DF}=1 & \mathrm{P}=0.011 & \text { (adjusted for ties) }\end{array}\end{array}$

Kruskal-Wallis Test: Density versus Top/Bottom

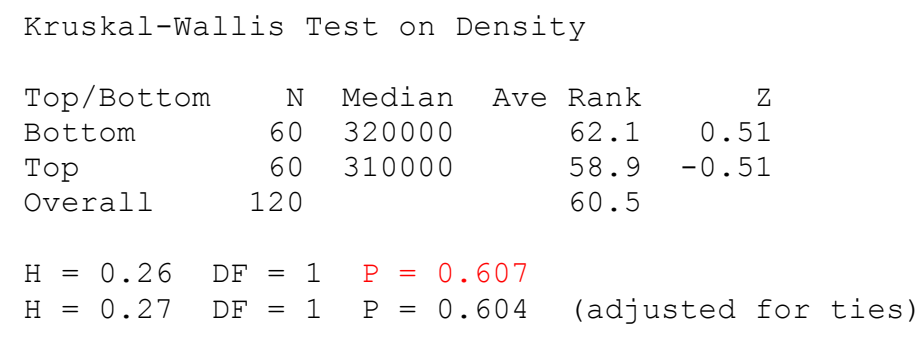

Figure J.1. Minitab output for cell density comparisons throughout L-shaped BVMs. pvalues are written in red. A statistically significant difference existed between cell densities in the 5 regions along the length of L-shaped BVMs $(p=0.000)$, between the cell densities in the inner and outer halves of L-shaped BVMs $(p=0.011)$, but not between the cell densities in the top and bottom halves of L-shaped BVMs $(p=0.607)$. 
One-way ANOVA: Density versus Region

$\begin{array}{lrrrrr}\begin{array}{lrrr}\text { Source } \\ \text { Region }\end{array} & \text { DF } & \text { S } & \text { MS } & \text { F } & P \\ \text { Error } & 95 & 18514000000 & 4628500000 & 3.71 & 0.008 \\ \text { Total } & 99 & 1.37059 \mathrm{E}+11 & & & \\ \mathrm{~S}=35325 & \mathrm{R}-\mathrm{Sq}=13.51 \% & \mathrm{R}-\mathrm{Sq}(\text { adj })=9.87 \%\end{array}$

One-way ANOVA: Density versus Inner/Outer

$\begin{array}{lrrrrr}\text { Source } & \text { DF } & \text { SS } & \text { MS } & F & P \\ \text { Inner/Outer } & 1 & 4988166667 & 4988166667 & 3.70 & 0.057 \\ \text { Error } & 98 & 1.32071 \mathrm{E}+11 & 1347661565 & & \\ \text { Total } & 99 & 1.37059 \mathrm{E}+11 & & & \\ \text { S }=36711 & \text { R-Sq }=3.64 \% & \text { R-Sq }(\text { adj })=2.66 \% & \end{array}$

\section{One-way ANOVA: Density versus Top/Bottom}

$$
\begin{array}{lrrrrr}
\text { Source } & \text { DF } & \text { SS } & \text { MS } & \text { F } & \text { P } \\
\text { Top/Bottom } & 1 & 81000000 & 81000000 & 0.06 & 0.810 \\
\text { Error } & 98 & 1.36978 \mathrm{E}+11 & 1397734694 & & \\
\text { Total } & 99 & 1.37059 \mathrm{E}+11 & & & \\
\mathrm{~S}=37386 & \mathrm{R}-\mathrm{Sq}=0.06 \% & \mathrm{R}-\mathrm{Sq}(\mathrm{adj})=0.00 \% &
\end{array}
$$

Figure J.2. Minitab output for cell density comparisons throughout U-shaped BVMs. pvalues are written in red. A statistically significant difference existed between cell densities in the 5 regions along the length of L-shaped BVMs $(p=0.008)$ but not between the cell densities in the inner and outer halves of L-shaped BVMs $(p=0.057)$ nor between the cell densities in the top and bottom halves of L-shaped BVMs $(p=0.810)$. 


\section{Appendix K: SEM Imaging Protocol}

\section{Purpose}

The following protocol was followed when carrying out any SEM imaging throughout the research described in this thesis.

\section{Components}

- $100 \%$ Ethanol

- Distilled water

- Miniature glass jars

- Forceps

- Pipet-Aid

- Pipettes

\section{Protocol}

1. Prepare the following solutions of ethanol by diluting $100 \%$ ethanol with water: $25 \%$ ethanol, $50 \%$ ethanol, $70 \%$ ethanol, $90 \%$ ethanol.

2. Allot one miniature glass jar for each dilution, and place the dilutions in their respective jars. Prepare two jars for $100 \%$ ethanol.

3. Place $2 \mathrm{~mL}$ of each dilution into its respective jar.

4. Dehydrate BVM samples by placing samples in each diltuion for 5 minutes starting with $25 \%$ ethanol. Be careful not to disrupt samples or tip of miniature glass jars.

5. Place samples in dessicator overnight.

6. Image samples using Hitachi TM-1000 SEM. 


\section{Appendix L: Histology and White Light Imaging Protocol}

\section{Purpose}

The following protocol was followed when carrying out histology and white light imaging of histology samples throughout the research described in this thesis.

\section{Components}

Histology reagents

Olympus BX41 microscope

\section{Protocol}

After embedding samples in paraffin, sectioning samples, and placing samples in oven, run samples through the following reagents for the specified times.

1. $3 \mathrm{~min}$ - xylene 1

2. $3 \mathrm{~min}-$ xylene 2

3. $1 \mathrm{~min}-\mathrm{Air}$

4. $2 \min -100 \%$

5. $2 \min -100 \%$

6. $2 \min -95 \%$

7. $2 \mathrm{~min}-\mathrm{Air}$

8. 4 min - Hematoxylin

9. $1 \mathrm{~min}-\mathrm{H}_{2} \mathrm{O}$ (distilled)

10. $30 \mathrm{sec}$ - Clarifier

11. 1 min - Bluing

12. $1 \mathrm{~min}-95 \%$

13. $1 \mathrm{~min} 30 \mathrm{sec}-$ Eosin

14. $1 \mathrm{~min}-100 \%$

15. $1 \mathrm{~min}-\mathrm{Air}$

16. $1 \mathrm{~min}$ - xylene 1

17. $1 \mathrm{~min}$ - xylene 2

Mount samples on slides, place coverslips on slides, and allow slides to dry for 2 days.

With permission or help from Dr. Cardinal, use the white-light microscope to obtain images of samples mounted on slides. The following steps detail operation of the whitelight microscope and correspond to figure L.1.

1. Log into notebook (white; initials; date; time and lamp hours).

2. Turn turret to setting 1 .

3. Turn on Optiscan wheels (black switch).

4. Turn on camera (black switch).

5. Set filter wheel 2 to 5 . 
6. Open shutter: o

7. Set Prior keypad to shutter S1.

8. Turn on white light (black switch on side of microscope arm).

9. Dial objectives to desired magnification (typically 10x for histology images of cellsodded scaffolds).

10. Set thin bar to icon of eye and camera.

11. Sign into computer by clicking Dr. Cardinal's account (password can be obtained from Dr. Cardinal).

12. Click QCapture Pro (on desktop).

13. Click camera icon at top of QCapture Pro window:

14. Place slide on microscope. Use joystick to move sample under microscope lens.

15. See figures L.2 and L.3.

16. Use dial on base of microscope to adjust exposure of live image.

17. Use course focus and fine focus on base of microscope to clarify live image.

18. See figure L.4.

19. Refocus live image if desired. Click Snap to take pictures. Save pictures if desired (labeled with sample info and magnification).

20. Quit QCapture Pro. Log out of computer.

21. Shut down microscope by switching off Optiscan wheels, camera (on top of microscope), and white light (on side of microscope arm).

22. Log out of notebook.

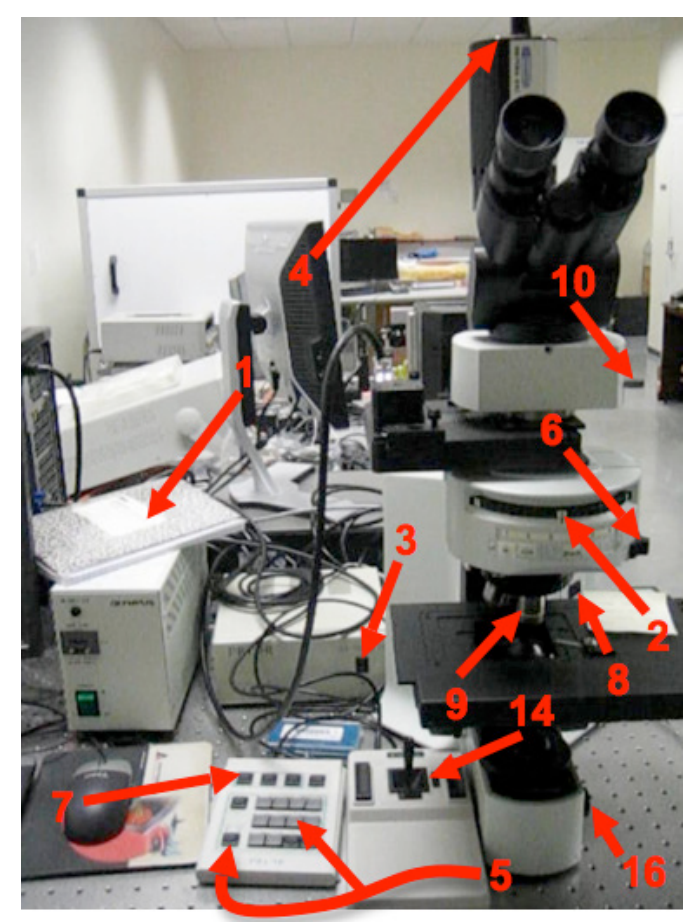

Figure L.1. Microscope anatomy. Numbers in figure correspond to steps above. 


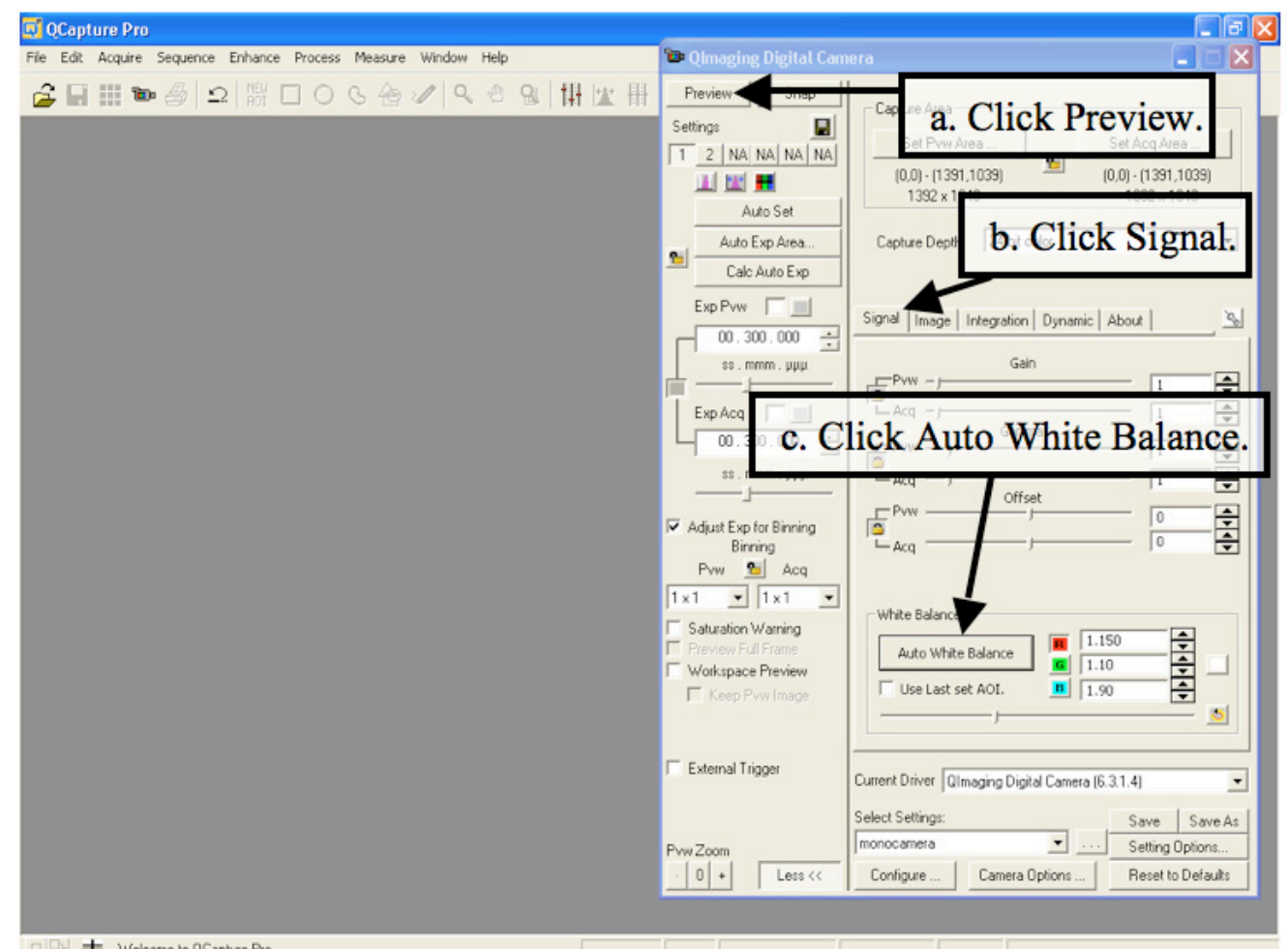

Figure L.2. QCapture Pro home window and control panel.

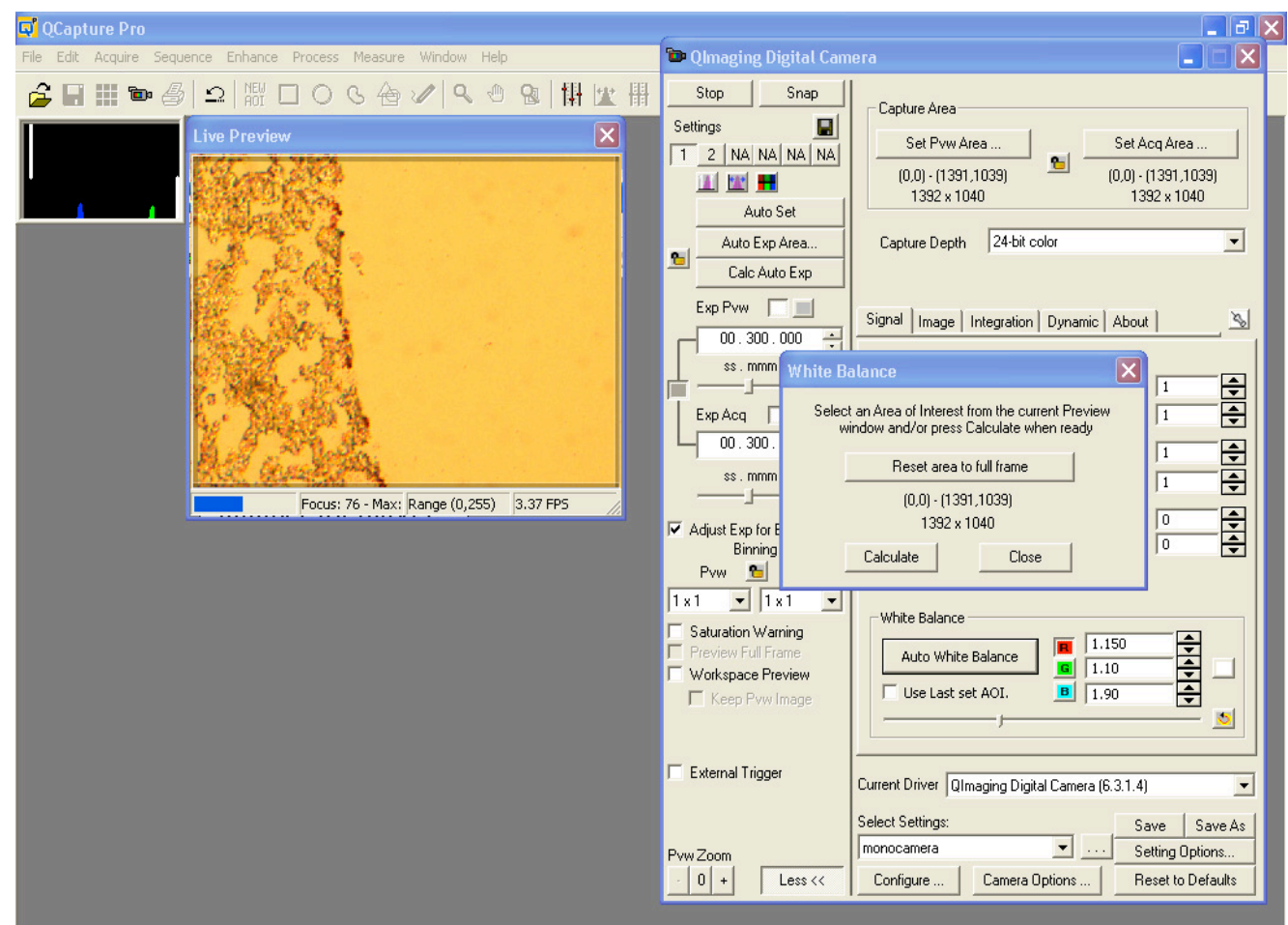

\pm Welcome to QCapture Pro

Figure L.3. QCapture Pro displays live image of sample. 


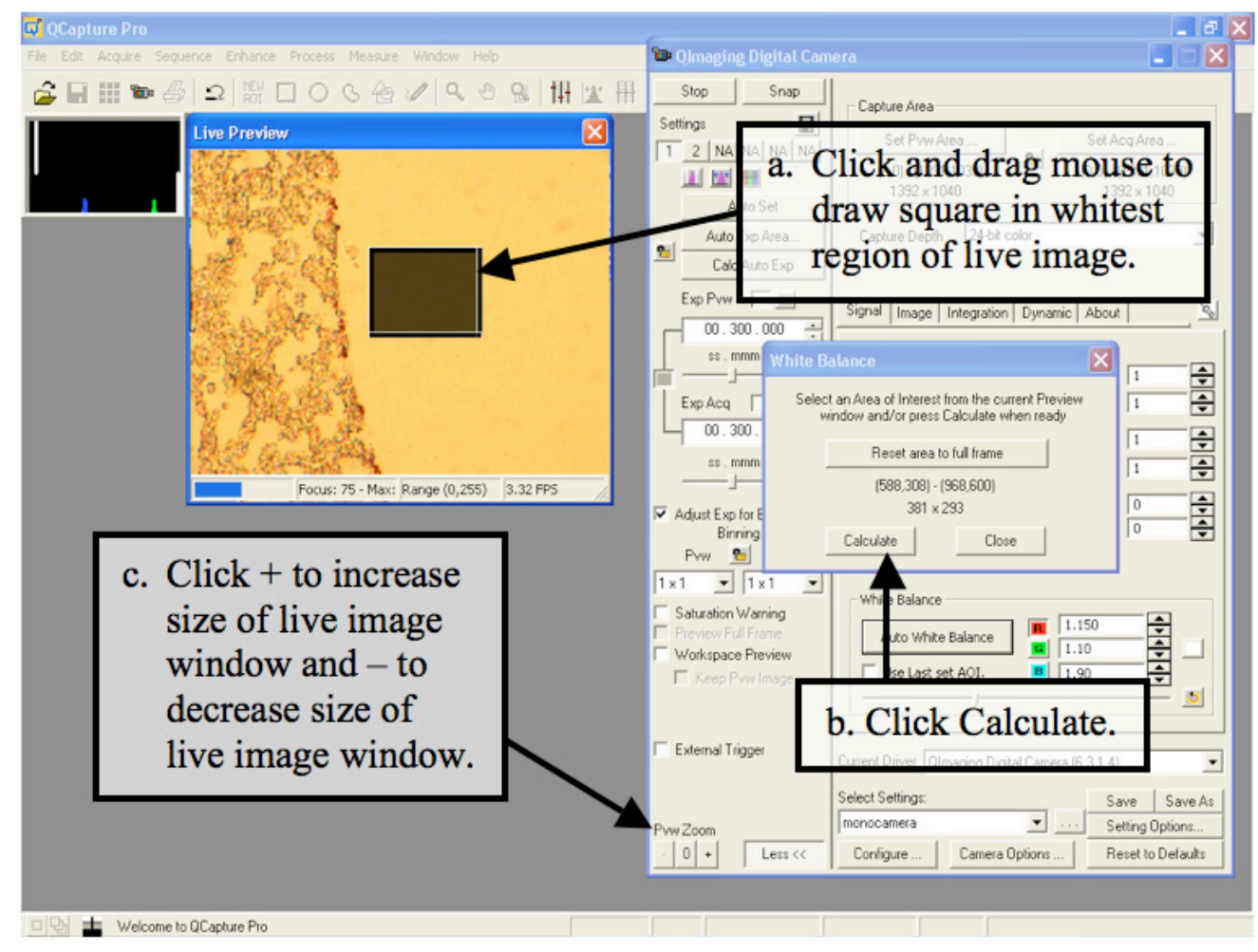

Figure L.4. QCapture Pro adjusts lighting of live image. 


\section{Appendix M: HUVSMC Media Preparation}

\section{Purpose}

The following was the list of components used to make HUVSMC media and the

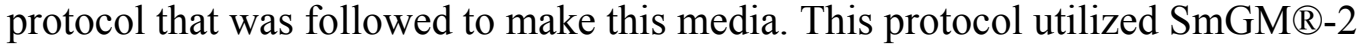
SingleQuots ${ }^{\circledR}$ (Lonza).

\section{Components}

- $500 \mathrm{~mL}$ Smooth muscle cell medium

- Other packaged components

\section{Protocol}

1. In a laminar flow hood, attach filter to bottle.

2. Attach vacuum to filter. Turn on vacuum.

3. Pour approximately $400 \mathrm{~mL}$ of smooth muscle cell medium into filter, leaving the remaining $100 \mathrm{~mL}$ to wash down other components from sides of filter.

4. Add the remaining components into the filter, using a pipette to acquire the exact quantities of each component.

5. Wash the filter with the remaining $100 \mathrm{~mL}$ of smooth muscle cell medium.

6. Detach the filter from the bottle. Cap the bottle. Place tape label on bottle that includes "HUVSMC Media," date, and initials.

7. Place labeled bottle in refrigerator $\left(4^{\circ} \mathrm{C}\right)$.

8. Before using media from refrigerator, warm media to $37^{\circ} \mathrm{C}$ in water bath. 


\section{Appendix N: Cell Tracker ${ }^{\mathrm{TM}}$ Preparation}

\section{Purpose}

This appendix includes the components and protocol used to prepare the Cell Tracker ${ }^{\mathrm{TM}}$ solutions that were used during the tissue-engineering of dual-sodded U-shaped BVMs in Chapter 6.

\section{Components}

- 5-20 $\mu \mathrm{L}$ pipette and sterile head

- 3-mL syringe with needle tip

- DMSO 100-mL bottle (anhydrous, Sigma-Aldrich, catalog number 276855)

- Cell Tracker ${ }^{\mathrm{TM}}$ Red (Invitrogen, Carlsbad, CA, catalog number C34522)

- Cell Tracker ${ }^{\mathrm{TM}}$ Green (Invitrogen, Carlsbad, CA catalog number C7025)

- Sterile serum-free media

- Pipettes

\section{Protocol}

1. Warm media in water bath.

2. Use needle tip and syringe to pull out $1 \mathrm{~mL}$ of DMSO, and place in $15-\mathrm{mL}$ conical.

3. Use micropipette to pull out $10.8 \mu \mathrm{L}$ of DMSO from $15-\mathrm{mL}$ conical.

4. Add DMSO to vial of Cell Tracker ${ }^{\mathrm{TM}}$. Attempt to get most of powdered Cell Tracker ${ }^{\mathrm{TM}}$ in with DMSO. This is a $10-\mathrm{mM}$ concentration of stock solution.

5. Dilute stock solution to desired concentration to create working solution. Use equation $\mathrm{c}_{1} \mathrm{v}_{1}=\mathrm{c}_{2} \mathrm{v}_{2}$, where $\mathrm{c}_{1}=10 \mathrm{mM}$ (from stock solution), $\mathrm{v}_{1}=$ unknown, $\mathrm{c}_{2}=$ desired concentration, and $\mathrm{v}_{2}=$ final volume needed.

6. Vortex working solution for use. Working solution can be stored in fridge for up to one week.

7. Remove cell media from flasks of cells.

8. Add working solution to flasks of cells.

9. Incubate flasks containing Cell Tracker ${ }^{\mathrm{TM}}$ Green for 30 minutes and flasks containing Cell Tracker ${ }^{\mathrm{TM}}$ Red for 15 minutes.

10. Remove working solution from flasks of cells.

11. Add cell media back into flasks of cells.

12. Incubate flasks for at least 30 minutes. 


\section{Appendix O: Protocol for Developing Dual-Sodded Complex-Shaped BVMs}

\section{Purpose}

The following protocol was used to tissue-engineer dual-sodded U-shaped BVMs. This protocol has not been used to tissue-engineer any other complex-shaped BVM.

\section{Components}

- $\quad$ ePTFE scaffold(s)

- 1.5 million HUVSMCs $/ \mathrm{cm}^{2}$ of scaffold

- 1.5 million HUVECs $/ \mathrm{cm}^{2}$ of scaffold

- Conditioning media (See Appendix E)

- Bioreactor media (See Appendix D)

- HUVEC media (See Appendix C)

- HUVSMC media (See Appendix M)

- Cell Tracker ${ }^{\mathrm{TM}}$

- Sterile $70 \%$ EtOH and sterile $100 \% \mathrm{EtOH}$

- Silk Suture

- Forceps

- Degassing flask with rubber stopper

- Multifunctional bioreactor (including 3 luer plugs and 2 rotating male-to-male luer lock connectors)

- Peristaltic pump

- Sterile gloves

\section{Protocol}

Preparation (Approximately 1.5 weeks prior to "Setup")

1. Wash all multifunctional bioreactors, bioreactor lids, and two-port lids with water, and dry overnight.

2. Sterilize multifunctional bioreactors, bioreactor lids, and two-port lids by placing them in individual sterilization bags and taking them to the Cal Poly Vet Clinic for EtO gassing.

3. Thaw, plan out calendar of cell culture, and begin cell culture.

4. Cut ePTFE scaffolds to the dimensions described in Section 2.3.2, and wear gloves while doing so.

Preparation (Approximately 1 day prior to "Setup")

5. Sterilize ePTFE scaffolds, forceps, and degassing flask

6. Make media (listed in components list above).

7. Degass conditioning media

8. Denucleate scaffolds by first placing them in $70 \% \mathrm{EtOH}$ for 10 minutes and then $100 \% \mathrm{EtOH}$ for 10 minutes.

9. Protein-coat scaffolds by placing them in the degassed conditioning media overnight in the small incubator at $37^{\circ} \mathrm{C}$. 
"Setup" Day

10. Warm up bioreactor media, conditioning media, HUVEC media, HUVSMC media.

11. Prepare Cell Tracker ${ }^{\mathrm{TM}}$ Green and Red solutions.

12. Fill biochamber with bioreactor media.

13. Insert sterile graft into biochamber.

14. Number biochamber lids with tape and marker.

15. Using syringe, flush lumen with conditioning media to prime graft.

a. Clamp lumen and continue to prime graft.

b. Repeat for all grafts.

16. Prime 2-port reservoirs with conditioning media.

17. Attach primed biochamber to 2-port reservoir and condition graft for 10 minutes.

a. Flow through lumen first to remove air, then clamp lumen and condition transmurally on 150-rpm setting.

18. Leave primed biochambers in large incubator until ready for sodding step.

19. Prime 2-port reservoirs with 75\% HUVEC media and 25\% HUVSMC media.

a. Prepare one for each vessel.

b. Be sure that drip is visible and outlet is submerged.

c. Clamp tubing and leave in big incubator.

20. Take primed biochambers and reservoirs to hood.

21. Attach outlet of reservoir to inlet stopcock of biochamber.

a. Leave reservoir inlet unattached and biochamber outlet facing trough.

22. Place $9 \mathrm{~mL}$ of Cell Tracker ${ }^{\mathrm{TM}}$ Green solution in $15 \mathrm{~mL}$ conical.

23. Vortex solution.

24. Remove HUVSMC media from T225 of HUVSMCs.

25. Add $9 \mathrm{~mL}$ of Cell Tracker ${ }^{\mathrm{TM}}$ Green solution to T225 of HUVSMCs (to cover total surface area of T225).

26. Place T225 in incubator for 30 minutes.

27. Remove Cell Tracker ${ }^{\mathrm{TM}}$ Green solution from T225.

28. Add $9 \mathrm{~mL}$ HUVSMC media to T225 (to keep cells alive before sodding).

29. Incubate T225 for 30 minutes.

30. Harvest HUVSMCs from T225.

a. Apply trypsin; deactivate with HUVSMC media.

31. Pellet cell suspension (on 4 for 4 minutes).

32. Resuspend pellet in $4 \mathrm{~mL}$ HUVSMC media.

33. Prepare one trough of $10 \mathrm{~mL}$ HUVSMC media and one trough of $4 \mathrm{~mL}$ cell suspension.

34. Prime tubing by capping off proximal stopcock so fluid will flow back into reservoir. Inject $5 \mathrm{~mL}$ HUVSMC media. Immediately replace stopcock to 45-degree angle.

Detach syringe.

35. Sod cells.

a. Take up $4 \mathrm{~mL}$ cell solution in syringe.

b. Slightly twist stopcock so fluid bulges out of tip of stopcock.

c. Attach syringe to stopcock.

d. Toggle stopcock to allow fluid to flow only distally through graft.

e. Inject $4 \mathrm{~mL}$ cell solution. 
f. While still pressuring syringe, cap off stopcock to 45-degree angle. Detach syringe.

36. Chase cells by repeating step 35 with $4 \mathrm{~mL}$ HUVSMC media instead of $4 \mathrm{~mL}$ cell solution.

37. Move BVM system to large incubator.

38. Maintain transmural flow at $10 \mathrm{rpm}$ for 30 minutes.

39. Repeat steps 22 through 39 for each bioreactor.

40. Place $9 \mathrm{~mL}$ of Cell Tracker ${ }^{\mathrm{TM}}$ Red solution in $15 \mathrm{~mL}$ conical.

41. Vortex solution.

42. Remove HUVEC media from T225 of HUVECs.

43. Add $9 \mathrm{~mL}$ of Cell Tracker ${ }^{\mathrm{TM}}$ Red solution to T225 of HUVECs (to cover total surface area of T225).

44. Place T225 in incubator for 15 minutes.

45. Remove Cell Tracker ${ }^{\mathrm{TM}}$ Red solution from T225.

46. Add $9 \mathrm{~mL}$ HUVEC media to T225 (to keep cells alive before sodding).

47. Incubate $\mathrm{T} 225$ for 30 minutes.

48. Harvest HUVECs from a T225.

a. Apply Trypsin; deactivate with HUVEC media.

49. Pellet cell suspension (on 4 for 4 minutes).

50. Resuspend pellet in $4 \mathrm{~mL}$ HUVEC media.

51. Prepare one trough of $10 \mathrm{~mL}$ HUVEC media and one trough of $4 \mathrm{~mL}$ cell suspension.

52. Prime tubing by capping off proximal stopcock so fluid will flow back into reservoir. Inject $5 \mathrm{~mL}$ HUVEC media. Immediately replace stopcock to 45-degree angle. Detach syringe.

53. Sod cells.

a. Take up $4 \mathrm{~mL}$ cell solution in syringe.

b. Slightly twist stopcock so fluid bulges out of tip of stopcock.

c. Attach syringe to stopcock.

d. Toggle stopcock to allow fluid to flow only distally through graft.

e. Inject $4 \mathrm{~mL}$ cell solution.

f. While still pressuring syringe, cap off stopcock to 45 -degree angle. Detach syringe.

54. Chase cells by repeating step 43 with $4 \mathrm{~mL}$ HUVEC media instead of $4 \mathrm{~mL}$ cell solution.

55. Move BVM system to large incubator.

56. Maintain transmural flow at $10 \mathrm{rpm}$ for 30 minutes.

57. Stop transmural flow, and maintain luminal flow at $10 \mathrm{rpm}$ for 1 hour.

58. Increase luminal flow to $15 \mathrm{rpm}$, and maintain for 1 hour.

59. Increase luminal flow to $20 \mathrm{rpm}$, and maintain overnight.

60. Take down 1-day BVMs.

61. Increase flow by $15 \mathrm{rpm}$ every hour to reach $150 \mathrm{rpm}$.

62. Take down 4-day BVMs. 


\title{
Appendix P: Copyright Reprint Permissions
}

\section{Figure 1.1 A and B Reprint Permissions}

\author{
$4 / 14 / 12$ \\ Rightslink Printable License \\ JOHN WILEY AND SONS LICENSE
}

TERMS AND CONDITIONS

Apr 14, 2012

This is a License Agreement between Robert D Chavez ("You") and John Wiley and Sons ("John Wiley and Sons") provided by Copyright Clearance Center ("CCC"). The license consists of your order details, the terms and conditions provided by John Wiley and Sons, and the payment terms and conditions.

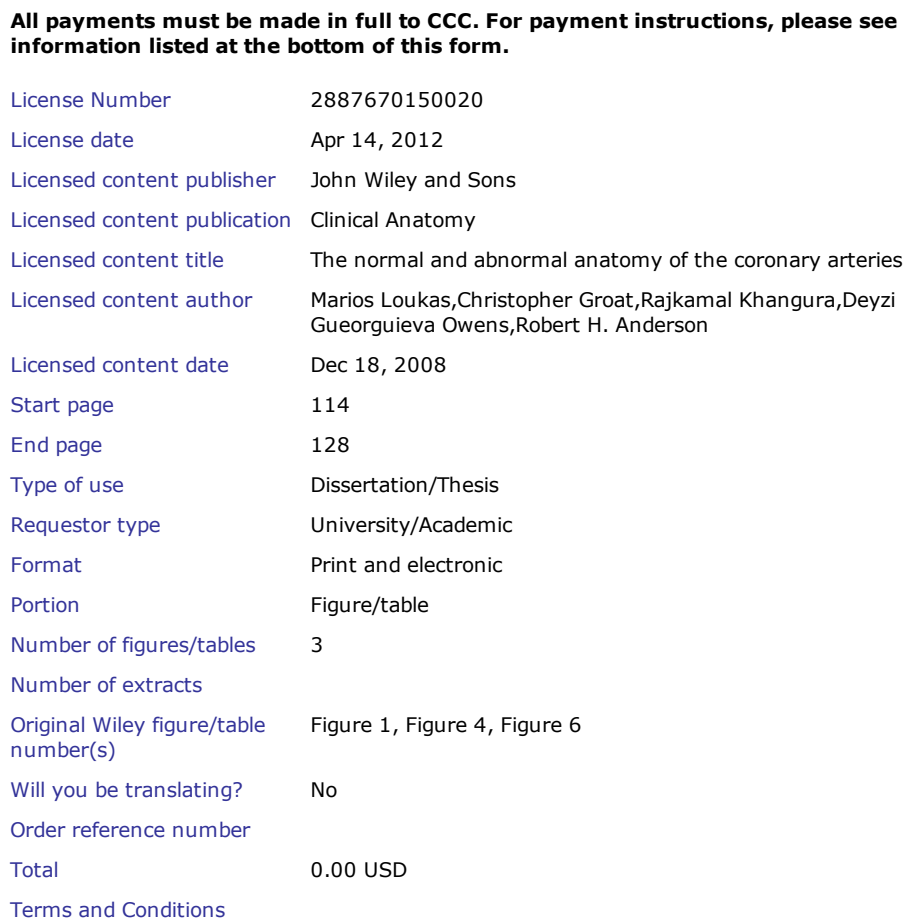

\section{TERMS AND CONDITIONS}

This copyrighted material is owned by or exclusively licensed to John Wiley \& Sons, Inc. or one of its group companies (each a "Wiley Company") or a society for whom a Wiley Company has exclusive publishing rights in relation to a particular journal (collectively WILEY"). By clicking "accept" in connection with completing this licensing transaction, you agree that the following terms and the billing and payment terms and conditions established by the Copyright Clearance Center Inc., ("CCC's Billing and Payment terms and
conditions"), at the time that you opened your Rightslink account (these are available at any tim https://s100.copyright.com/AppDispatchServlet 
at http://myaccount.copyright.com)

Terms and Conditions

1. The materials you have requested permission to reproduce (the "Materials") are protected by copyright.

2. You are hereby granted a personal, non-exclusive, non-sublicensable, non-transferable worldwide, limited license to reproduce the Materials for the purpose specified in the licensing process. This license is for a one-time use only with a maximum distribution equal to the number that you identified in the licensing process. Any form of republication granted by this licence must be completed within two years of the date of the grant of this licence (although copies prepared before may be distributed thereafter). The Materials shall not be used in any other manner or for any other purpose. Permission is granted subject to an appropriate acknowledgement given to the author, title of the material/book/journal and the publisher. You shall also duplicate the copyright notice that appears in the Wiley publication in your use of the Material. Permission is also granted on the understanding that nowhere in the text is a previously published source acknowledged for all or part of this Material. Any third party material is expressly excluded from this permission.

3. With respect to the Materials, all rights are reserved. Except as expressly granted by the terms of the license, no part of the Materials may be copied, modified, adapted (except for minor reformatting required by the new Publication), translated, reproduced, transferred or distributed, in any form or by any means, and no derivative works may be made based on the Materials without the prior permission of the respective copyright owner. You may not alter, remove or suppress in any manner any copyright, trademark or other notices displayed by the Materials. You may not license, rent, sell, loan, lease, pledge, offer as security, transfer or assign the Materials, or any of the rights granted to you hereunder to any other person.

4. The Materials and all of the intellectual property rights therein shall at all times remain the exclusive property of John Wiley \& Sons Inc or one of its related companies (WILEY) or their respective licensors, and your interest therein is only that of having possession of and the right to reproduce the Materials pursuant to Section 2 herein during the continuance of this Agreement. You agree that you own no right, title or interest in or to the Materials or any of the intellectual property rights therein. You shall have no rights hereunder other than the license as provided for above in Section 2. No right, license or interest to any trademark, trade name, service mark or other branding ("Marks") of WILEY or its licensors is granted hereunder, and you agree that you shall not assert any such right, license or interest with respect thereto.

5. NEITHER WILEY NOR ITS LICENSORS MAKES ANY WARRANTY OR REPRESENTATION OF ANY KIND TO YOU OR ANY THIRD PARTY, EXPRESS, IMPLIED OR STATUTORY, WITH RESPECT TO THE MATERIALS OR THE ACCURACY OF ANY INFORMATION CONTAINED IN THE MATERIALS, INCLUDING, WITHOUT LIMITATION, ANY IMPLIED WARRANTY OF MERCHANTABILITY, ACCURACY, SATISFACTORY QUALITY, FITNESS FOR A PARTICULAR PURPOSE, USABILITY, INTEGRATION OR NON-INFRINGEMENT AND ALL SUCH WARRANTIES ARE HEREBY EXCLUDED BY WILEY AND ITS LICENSORS AND WAIVED BY YOU.

6. WILEY shall have the right to terminate this Agreement immediately upon breach of this Agreement by you.

7. You shall indemnify, defend and hold harmless WILEY, its Licensors and their respective directors, officers, agents and employees, from and against any actual or threatened claims, demands, causes of action or proceedings arising from any breach of this Agreement by you.

8. IN NO EVENT SHALL WILEY OR ITS LICENSORS BE LIABLE TO YOU OR ANY OTHER PARTY OR ANY OTHER PERSON OR ENTITY FOR ANY SPECIAL, CONSEQUENTIAL, INCIDENTAL, INDIRECT, EXEMPLARY OR PUNITIVE DAMAGES, HOWEVER CAUSED, ARISING OUT OF OR IN CONNECTION WITH THE DOWNLOADING, PROVISIONING, VIEWING OR USE OF THE MATERIALS REGARDLESS OF THE FORM OF ACTION, WHETHER FOR BREACH OF CONTRACT, BREACH OF WARRANTY, TORT, NEGLIGENCE, INFRINGEMENT OR OTHERWISE (INCLUDING, WITHOUT LIMITATION, DAMAGES BASED ON LOSS OF PROFITS, DATA, FILES, USE, BUSINESS OPPORTUNITY OR CLAIMS OF THIRD PARTIES), AND WHETHER OR NOT THE PARTY HAS BEEN ADVISED OF THE POSSIBILITY OF SUCH 
DAMAGES. THIS LIMITATION SHALL APPLY NOTWITHSTANDING ANY FAILURE OF ESSENTIAL PURPOSE OF ANY LIMITED REMEDY PROVIDED HEREIN.

9. Should any provision of this Agreement be held by a court of competent jurisdiction to be illegal, invalid, or unenforceable, that provision shall be deemed amended to achieve as nearly as possible the same economic effect as the original provision, and the legality, validity and enforceability of the remaining provisions of this Agreement shall not be affected or impaired thereby.

10. The failure of either party to enforce any term or condition of this Agreement shall not constitute a waiver of either party's right to enforce each and every term and condition of this Agreement. No breach under this agreement shall be deemed waived or excused by either party unless such waiver or consent is in writing signed by the party granting such waiver or consent. The waiver by or consent of a party to a breach of any provision of this Agreement shall not operate or be construed as a waiver of or consent to any other or subsequent breach by such other party.

11. This Agreement may not be assigned (including by operation of law or otherwise) by you without WILEY's prior written consent.

12. Any fee required for this permission shall be non-refundable after thirty (30) days from receipt.

13. These terms and conditions together with CCC's Billing and Payment terms and conditions (which are incorporated herein) form the entire agreement between you and WILEY concernin this licensing transaction and (in the absence of fraud) supersedes all prior agreements and representations of the parties, oral or written. This Agreement may not be amended except in writing signed by both parties. This Agreement shall be binding upon and inure to the benefit of the parties' successors, legal representatives, and authorized assigns.

14. In the event of any conflict between your obligations established by these terms and conditions and those established by CCC's Billing and Payment terms and conditions, these terms and conditions shall prevail.

15. WILEY expressly reserves all rights not specifically granted in the combination of (i) the license details provided by you and accepted in the course of this licensing transaction, (ii) these terms and conditions and (iii) CCC's Billing and Payment terms and conditions.

16. This Agreement will be void if the Type of Use, Format, Circulation, or Requestor Type was misrepresented during the licensing process.

17. This Agreement shall be governed by and construed in accordance with the laws of the State of New York, USA, without regards to such state's conflict of law rules. Any legal action, suit or proceeding arising out of or relating to these Terms and Conditions or the breach thereof shall be instituted in a court of competent jurisdiction in New York County in the State of New York in the United States of America and each party hereby consents and submits to the personal jurisdiction of such court, waives any objection to venue in such court and consents to service of process by registered or certified mail, return receipt requested, at the last known address of such party.

\section{Wiley Open Access Terms and Conditions}

All research articles published in Wiley Open Access journals are fully open access: immediately freely available to read, download and share. Articles are published under the terms of the Creative Commons Attribution Non Commercial License. which permits use, distribution and reproduction in any medium, provided the original work is properly cited and is not used for commercial purposes. The license is subject to the Wiley Open Access terms and conditions: Wiley Open Access articles are protected by copyright and are posted to repositories and websites in accordance with the terms of the Creative Commons Attribution Non Commercial License. At the time of deposit, Wiley Open Access articles include all changes made during peer review, copyediting, and publishing. Repositories and websites that host the article are responsible for incorporating any publisher-supplied amendments or retractions issued subsequently.

Wiley Open Access articles are also available without charge on Wiley's publishing platform, Wiley Online Library or any successor sites. 
For non-commercial and non-promotional purposes individual users may access, download, copy, display and redistribute to colleagues Wiley Open Access articles, as well as adapt, translate, textand data-mine the content subject to the following conditions:

- The authors' moral rights are not compromised. These rights include the right of "paternity" (also known as "attribution" - the right for the author to be identified as such) and "integrity" (the right for the author not to have the work altered in such a way that the author's reputation or integrity may be impugned).

- Where content in the article is identified as belonging to a third party, it is the obligation of the user to ensure that any reuse complies with the copyright policies of the owner of that content.

- If article content is copied, downloaded or otherwise reused for non-commercial research and education purposes, a link to the appropriate bibliographic citation (authors, journal, article title, volume, issue, page numbers, DOI and the link to the definitive published version on Wiley Online Library) should be maintained. Copyright notices and disclaimers must not be deleted.

- Any translations, for which a prior translation agreement with Wiley has not been agreed, must prominently display the statement: "This is an unofficial translation of an article that appeared in a Wiley publication. The publisher has not endorsed this translation."

\section{Use by commercial "for-profit" organisations}

Use of Wiley Open Access articles for commercial, promotional, or marketing purposes requires further explicit permission from Wiley and will be subject to a fee. Commercial purposes include:

- Copying or downloading of articles, or linking to such articles for further redistribution, sale or licensing;

- Copying, downloading or posting by a site or service that incorporates advertising with such content;

- The inclusion or incorporation of article content in other works or services (other than normal quotations with an appropriate citation) that is then available for sale or licensing, for a fee (for example, a compilation produced for marketing purposes, inclusion in a sales pack)

- Use of article content (other than normal quotations with appropriate citation) by for-profit organisations for promotional purposes

- Linking to article content in e-mails redistributed for promotional, marketing or educational purposes;

- Use for the purposes of monetary reward by means of sale, resale, licence, loan, transfer or other form of commercial exploitation such as marketing products

- Print reprints of Wiley Open Access articles can be purchased from:

corporatesales@wiley.com

Other Terms and Conditions:

BY CLICKING ON THE "I AGREE..." BOX, YOU ACKNOWLEDGE THAT YOU HAVE READ AND FULLY UNDERSTAND EACH OF THE SECTIONS OF AND PROVISIONS SET FORTH IN THIS AGREEMENT AND THAT YOU ARE IN AGREEMENT WITH AND ARE WILLING TO ACCEPT ALL OF YOUR 
OBLIGATIONS AS SET FORTH IN THIS AGREEMENT.

v1.7

If you would like to pay for this license now, please remit this license along with your payment made payable to "COPYRIGHT CLEARANCE CENTER" otherwise you will be

invoiced within 48 hours of the license date. Payment should be in the form of a check or money order referencing your account number and this invoice number

RLNK500760434.

Once you receive your invoice for this order, you may pay your invoice by credit card.

Please follow instructions provided at that time.

Make Payment To:

Copyright Clearance Center

Dept 001

P.O. Box 843006

Boston, MA 02284-3006

For suggestions or comments regarding this order, contact RightsLink Customer

Support: customercare@copyright.com or +1-877-622-5543 (toll free in the US) or +1978-646-2777.

Gratis licenses (referencing $\$ 0$ in the Total field) are free. Please retain this printable license for your reference. No payment is required. 
Figure 1.2 A Reprint Permissions

$4 / 16 / 12$

Rightslink Printable License

SPRINGER LICENSE

TERMS AND CONDITIONS

Apr 17, 2012

This is a License Agreement between Robert D Chavez ("You") and Springer ("Springer") provided by Copyright Clearance Center ("CCC"). The license consists of your order details, the terms and conditions provided by Springer, and the payment terms and conditions.

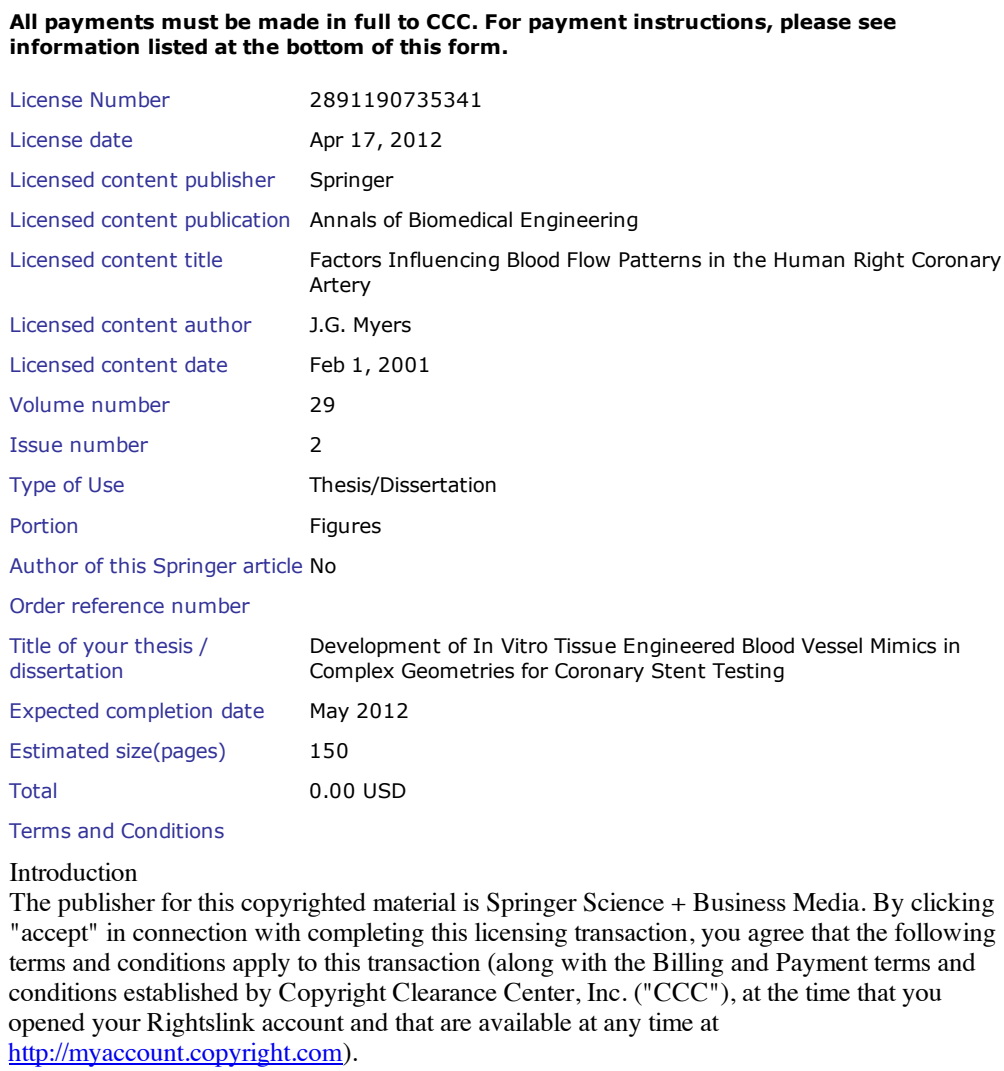


indicated in your enquiry.

Licenses are for one-time use only with a maximum distribution equal to the number that you identified in the licensing process

This License includes use in an electronic form, provided its password protected or on the university's intranet or repository, including UMI (according to the definition at the Sherpa website: http://www .sherpa.ac.uk/romeo/). For any other electronic use, please contact Springer at (permissions.dordrecht@ springer.com or permissions.heidelberg@springer.com).

The material can only be used for the purpose of defending your thesis, and with a maximum of 100 extra copies in paper.

Although Springer holds copyright to the material and is entitled to negotiate on rights, this license is only valid, provided permission is also obtained from the (co) author (address is given with the article/chapter) and provided it concerns original material which does not carry references to other sources (if material in question appears with credit to another source, authorization from that source is required as well).

Permission free of charge on this occasion does not prejudice any rights we might have to charge for reproduction of our copyrighted material in the future.

Altering/Modifying Material: Not Permitted

You may not alter or modify the material in any manner. Abbreviations, additions, deletions and/or any other alterations shall be made only with prior written authorization of the author(s) and/or Springer Science + Business Media. (Please contact Springer at (permissions.dordrecht@springer.com or permissions.heidelberg@springer.com)

Reservation of Rights

Springer Science + Business Media reserves all rights not specifically granted in the combination of (i) the license details provided by you and accepted in the course of this licensing transaction, (ii) these terms and conditions and (iii) CCC's Billing and Payment terms and conditions.

Copyright Notice:Disclaimer

You must include the following copyright and permission notice in connection with any reproduction of the licensed material: "Springer and the original publisher/journal title, volume, year of publication, page, chapter/article title, name(s) of author(s), figure number(s), original copyright notice) is given to the publication in which the material was originally published, by adding; with kind permission from Springer Science and Business Media"

Warranties: None

Example 1: Springer Science + Business Media makes no representations or warranties with respect to the licensed material.

Example 2: Springer Science + Business Media makes no representations or warranties with respect to the licensed material and adopts on its own behalf the limitations and disclaimers established by $\mathrm{CCC}$ on its behalf in its Billing and Payment terms and conditions for this licensing transaction.

Indemnity 
You hereby indemnify and agree to hold harmless Springer Science + Business Media and $\mathrm{CCC}$, and their respective officers, directors, employees and agents, from and against any and all claims arising out of your use of the licensed material other than as specifically authorized pursuant to this license.

No Transfer of License

This license is personal to you and may not be sublicensed, assigned, or transferred by you to any other person without Springer Science + Business Media's written permission.

No Amendment Except in Writing

This license may not be amended except in a writing signed by both parties (or, in the case of Springer Science + Business Media, by CCC on Springer Science + Business Media's behalf).

Objection to Contrary Terms

Springer Science + Business Media hereby objects to any terms contained in any purchase order, acknowledgment, check endorsement or other writing prepared by you, which terms are inconsistent with these terms and conditions or CCC's Billing and Payment terms and conditions. These terms and conditions, together with CCC's Billing and Payment terms and conditions (which are incorporated herein), comprise the entire agreement between you and Springer Science + Business Media (and CCC) concerning this licensing transaction. In the event of any conflict between your obligations established by these terms and conditions and those established by CCC's Billing and Payment terms and conditions, these terms and conditions shall control.

Jurisdiction

All disputes that may arise in connection with this present License, or the breach thereof, shal be settled exclusively by arbitration, to be held in The Netherlands, in accordance with Dutch law, and to be conducted under the Rules of the 'Netherlands Arbitrage Instituut' (Netherlands Institute of Arbitration).OR:

All disputes that may arise in connection with this present License, or the breach thereof, shall be settled exclusively by arbitration, to be held in the Federal Republic of Germany, in accordance with German law.

Other terms and conditions:

v1.3

If you would like to pay for this license now, please remit this license along with your payment made payable to "COPYRIGHT CLEARANCE CENTER" otherwise you will be invoiced within 48 hours of the license date. Payment should be in the form of a check or money order referencing your account number and this invoice number RLNK500761757.

Once you receive your invoice for this order, you may pay your invoice by credit card. Please follow instructions provided at that time.

Make Payment To:

Copyright Clearance Center

Dept 001

P.O. Box 843006

Boston, MA 02284-3006

For suggestions or comments regarding this order, contact RightsLink Custome Support: customercare@copyright.com or +1-877-622-5543 (toll free in the US) or +1978-646-2777.

https://s100.copyright.com/AppDispatchServlet 
Gratis licenses (referencing $\$ 0$ in the Total field) are free. Please retain this printable license for your reference. No payment is required. 


\title{
Figure 1.3 Reprint Permissions
}

\author{
$4 / 14 / 12$ \\ Rightslink Printable Licens \\ ELSEVIER LICENSE \\ TERMS AND CONDITIONS \\ Apr 14, 2012 \\ This is a License Agreement between Robert D Chavez ("You") and Elsevier ("Elsevier") \\ provided by Copyright Clearance Center ("CCC"). The license consists of your order details, \\ the terms and conditions provided by Elsevier, and the payment terms and conditions.

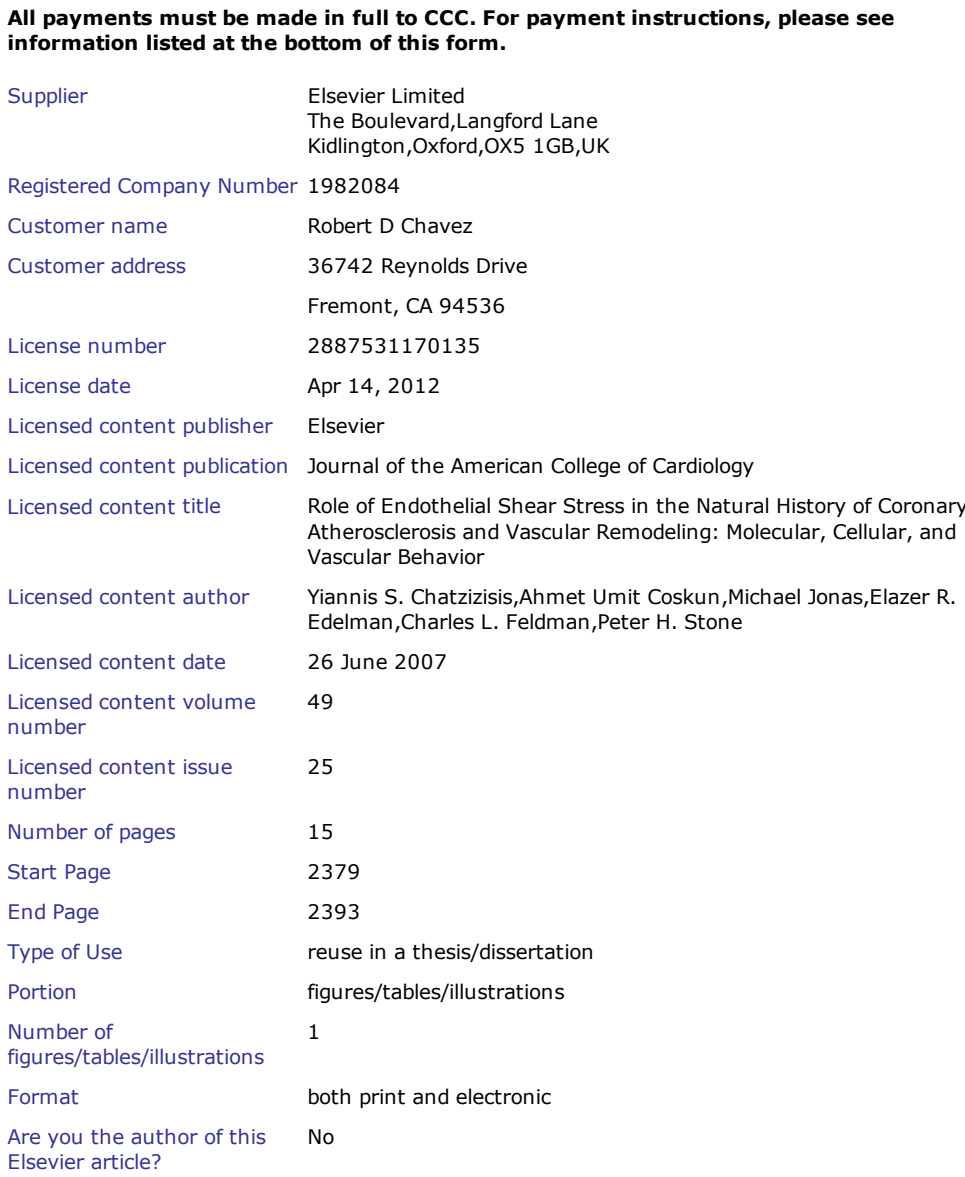




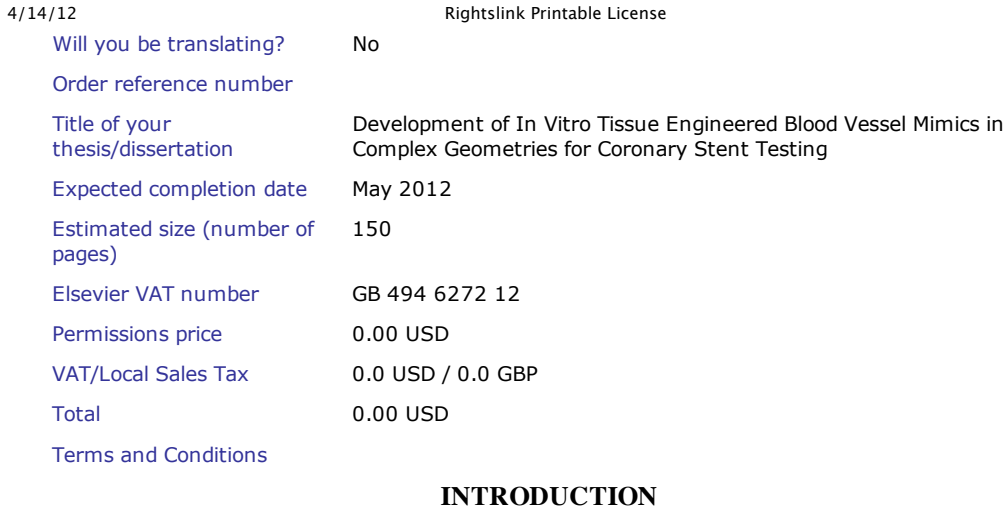

\section{INTRODUCTION}

1. The publisher for this copyrighted material is Elsevier. By clicking "accept" in connection with completing this licensing transaction, you agree that the following terms and conditions apply to this transaction (along with the Billing and Payment terms and conditions established by Copyright Clearance Center, Inc. ("CCC"), at the time that you opened your Rightslink account and that are available at any time at http://myaccount.copyright.com).

\section{GENERAL TERMS}

2. Elsevier hereby grants you permission to reproduce the aforementioned material subject to the terms and conditions indicated.

3. Acknowledgement: If any part of the material to be used (for example, figures) has appeared in our publication with credit or acknowledgement to another source, permission must also be sought from that source. If such permission is not obtained then that material may not be included in your publication/copies. Suitable acknowledgement to the source must be made, either as a footnote or in a reference list at the end of your publication, as follows:

"Reprinted from Publication title, Vol /edition number, Author(s), Title of article / title of chapter, Pages No., Copyright (Year), with permission from Elsevier [OR APPLICABLE SOCIETY COPYRIGHT OWNER]." Also Lancet special credit - "Reprinted from The Lancet, Vol. number, Author(s), Title of article, Pages No., Copyright (Year), with permission from Elsevier.'

4. Reproduction of this material is confined to the purpose and/or media for which permission is hereby given.

5. Altering/Modifying Material: Not Permitted. However figures and illustrations may be altered/adapted minimally to serve your work. Any other abbreviations, additions, deletions and/or any other alterations shall be made only with prior written authorization of Elsevier Ltd. (Please contact Elsevier at permissions@elsevier.com)

6. If the permission fee for the requested use of our material is waived in this instance, please be advised that your future requests for Elsevier materials may attract a fee.

7. Reservation of Rights: Publisher reserves all rights not specifically granted in the https://s100.copyright.com/AppDispatchServlet 
combination of (i) the license details provided by you and accepted in the course of this licensing transaction, (ii) these terms and conditions and (iii) $\mathrm{CCC}^{\prime}$ s Billing and Payment terms and conditions.

8. License Contingent Upon Payment: While you may exercise the rights licensed immediately upon issuance of the license at the end of the licensing process for the transaction, provided that you have disclosed complete and accurate details of your proposed use, no license is finally effective unless and until full payment is received from you (either by publisher or by $\mathrm{CCC}$ ) as provided in CCC's Billing and Payment terms and conditions. If full payment is not received on a timely basis, then any license preliminarily granted shall be deemed automatically revoked and shall be void as if never granted. Further, in the event that you breach any of these terms and conditions or any of CCC's Billing and Payment terms and conditions, the license is automatically revoked and shall be void as if never granted. Use of materials as described in a revoked license, as well as any use of the materials beyond the scope of an unrevoked license, may constitute copyright infringement and publisher reserves the right to take any and all action to protect its copyright in the materials.

9. Warranties: Publisher makes no representations or warranties with respect to the licensed material.

10. Indemnity: You hereby indemnify and agree to hold harmless publisher and CCC, and their respective officers, directors, employees and agents, from and against any and all claims arising out of your use of the licensed material other than as specifically authorized pursuant to this license.

11. No Transfer of License: This license is personal to you and may not be sublicensed, assigned, or transferred by you to any other person without publisher's written permission.

12. No Amendment Except in Writing: This license may not be amended except in a writing signed by both parties (or, in the case of publisher, by CCC on publisher's behalf).

13. Objection to Contrary Terms: Publisher hereby objects to any terms contained in any purchase order, acknowledgment, check endorsement or other writing prepared by you, which terms are inconsistent with these terms and conditions or CCC's Billing and Payment terms and conditions. These terms and conditions, together with CCC's Billing and Payment terms and conditions (which are incorporated herein), comprise the entire agreement between you and publisher (and CCC) concerning this licensing transaction. In the event of any conflict between your obligations established by these terms and conditions and those established by CCC's Billing and Payment terms and conditions, these terms and conditions shall control.

14. Revocation: Elsevier or Copyright Clearance Center may deny the permissions described in this License at their sole discretion, for any reason or no reason, with a full refund payable to you. Notice of such denial will be made using the contact information provided by you. Failure to receive such notice will not alter or invalidate the denial. In no event will Elsevier or Copyright Clearance Center be responsible or liable for any costs, expenses or damage incurred by you as a result of a denial of your permission request, other than a refund of the amount(s) paid by you to Elsevier and/or Copyright Clearance Center for denied permissions.

\section{LIMITED LICENSE}

The following terms and conditions apply only to specific license types: 
15. Translation: This permission is granted for non-exclusive world English rights only unless your license was granted for translation rights. If you licensed translation rights you may only translate this content into the languages you requested. A professional translator must perform all translations and reproduce the content word for word preserving the integrity of the article. If this license is to re-use 1 or 2 figures then permission is granted for nonexclusive world rights in all languages.

16. Website: The following terms and conditions apply to electronic reserve and author websites:

Electronic reserve: If licensed material is to be posted to website, the web site is to be password-protected and made available only to bona fide students registered on a relevant course if:

This license was made in connection with a course,

This permission is granted for 1 year only. You may obtain a license for future website posting,

All content posted to the web site must maintain the copyright information line on the bottom of each image,

A hyper-text must be included to the Homepage of the journal from which you are licensing at http://www.sciencedirect.com/science/journal/xxxxx or the Elsevier homepage for books at http://www.elsevier.com , and

Central Storage: This license does not include permission for a scanned version of the material to be stored in a central repository such as that provided by Heron/XanEdu.

17. Author website for journals with the following additional clauses:

All content posted to the web site must maintain the copyright information line on the bottom of each image, and

he permission granted is limited to the personal version of your paper. You are not allowed to download and post the published electronic version of your article (whether PDF or HTML,

proof or final version), nor may you scan the printed edition to create an electronic version,

A hyper-text must be included to the Homepage of the journal from which you are licensing at http://www.sciencedirect.com/science/journal/xxxxx , As part of our normal production process, you will receive an e-mail notice when your article appears on Elsevier's online service ScienceDirect (www.sciencedirect.com). That e-mail will include the article's Digital Object Identifier (DOI). This number provides the electronic link to the published article and should be included in the posting of your personal version. We ask that you wait until you receive this e-mail and have the DOI to do any posting.

Central Storage: This license does not include permission for a scanned version of the material to be stored in a central repository such as that provided by Heron/XanEdu.

18. Author website for books with the following additional clauses:

Authors are permitted to place a brief summary of their work online only.

A hyper-text must be included to the Elsevier homepage at http://www .elsevier.com

All content posted to the web site must maintain the copyright information line on the bottom of each image

You are not allowed to download and post the published electronic version of your chapter, nor may you scan the printed edition to create an electronic version.

Central Storage: This license does not include permission for a scanned version of the material to be stored in a central repository such as that provided by Heron/XanEdu. 
19. Website (regular and for author): A hyper-text must be included to the Homepage of the journal from which you are licensing at http://www.sciencedirect.com/science/journal/xxxxx. or for books to the Elsevier homepage at http://www.elsevier.com

20. Thesis/Dissertation: If your license is for use in a thesis/dissertation your thesis may be submitted to your institution in either print or electronic form. Should your thesis be published commercially, please reapply for permission. These requirements include permission for the Library and Archives of Canada to supply single copies, on demand, of the complete thesis and include permission for UMI to supply single copies, on demand, of the complete thesis. Should your thesis be published commercially, please reapply for permission.

\section{Other Conditions:}

v1.6

If you would like to pay for this license now, please remit this license along with your payment made payable to "COPYRIGHT CLEARANCE CENTER" otherwise you will be

invoiced within $\mathbf{4 8}$ hours of the license date. Payment should be in the form of a check or money order referencing your account number and this invoice number

RLNK500760402.

Once you receive your invoice for this order, you may pay your invoice by credit card. Please follow instructions provided at that time.

Make Payment To:

Copyright Clearance Center

Dept 001

P.O. Box 843006

Boston, MA 02284-3006

For suggestions or comments regarding this order, contact RightsLink Custome Support: customercare@copyright.com or +1-877-622-5543 (toll free in the US) or +1978-646-2777.

Gratis licenses (referencing $\$ 0$ in the Total field) are free. Please retain this printable license for your reference. No payment is required. 


\section{Figure 1.4 Reprint Permissions}

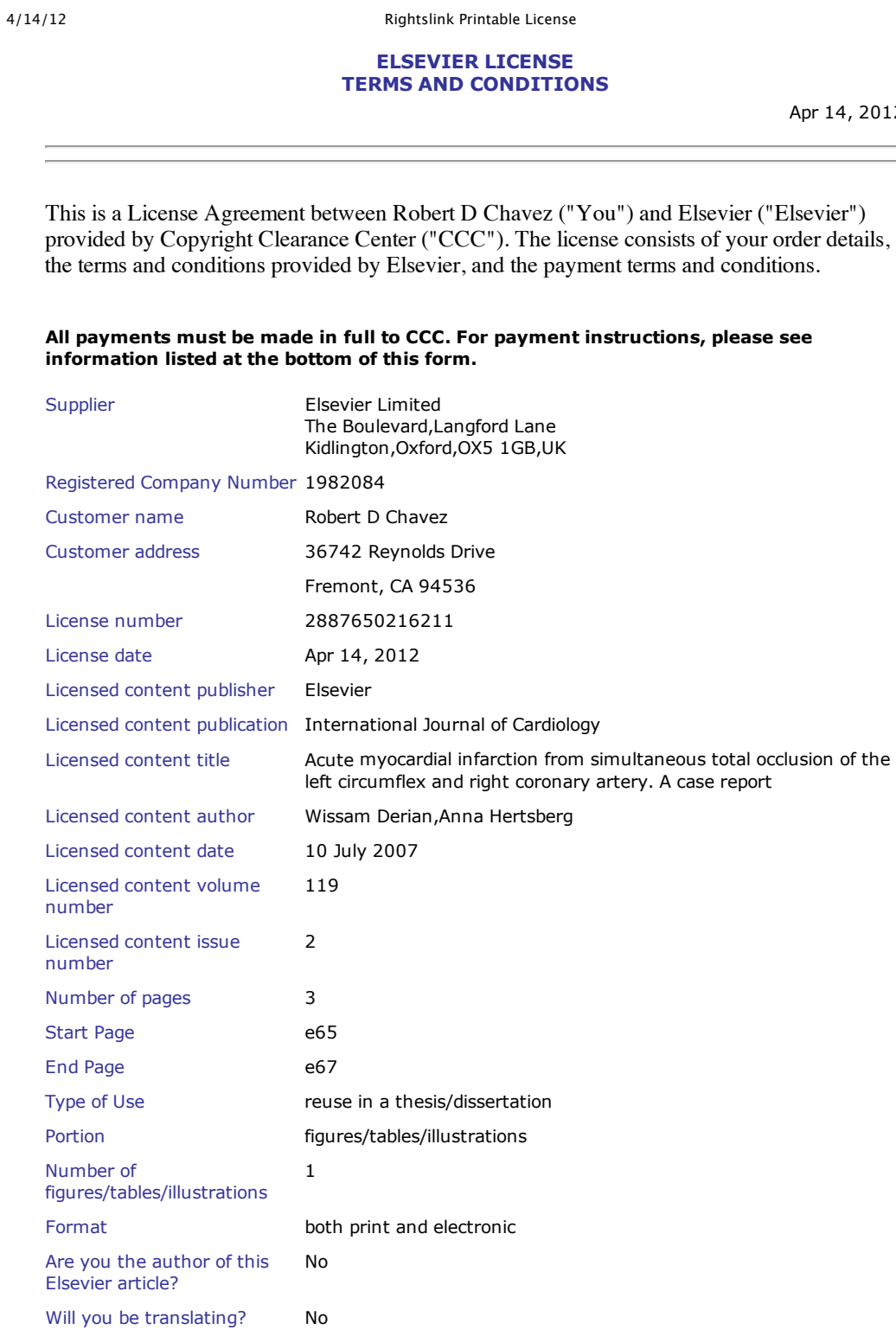




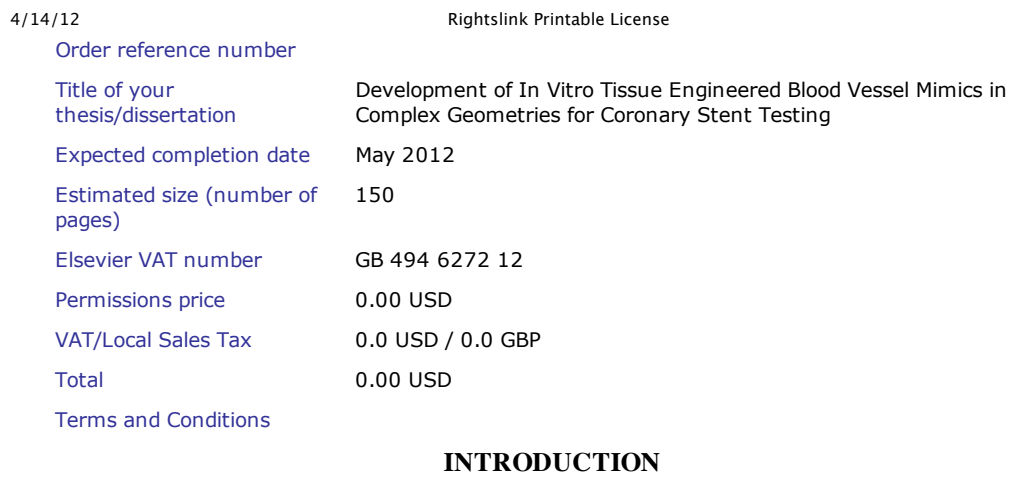

\section{INTRODUCTION}

1. The publisher for this copyrighted material is Elsevier. By clicking "accept" in connection with completing this licensing transaction, you agree that the following terms and conditions apply to this transaction (along with the Billing and Payment terms and conditions established by Copyright Clearance Center, Inc. ("CCC"), at the time that you opened your Rightslink account and that are available at any time at http://myaccount.copyright.com).

\section{GENERAL TERMS}

2. Elsevier hereby grants you permission to reproduce the aforementioned material subject to the terms and conditions indicated.

3. Acknowledgement: If any part of the material to be used (for example, figures) has appeared in our publication with credit or acknowledgement to another source, permission must also be sought from that source. If such permission is not obtained then that material may not be included in your publication/copies. Suitable acknowledgement to the source must be made, either as a footnote or in a reference list at the end of your publication, as follows:

"Reprinted from Publication title, Vol /edition number, Author(s), Title of article / title of chapter, Pages No., Copyright (Year), with permission from Elsevier [OR APPLICABLE SOCIETY COPYRIGHT OWNER]." Also Lancet special credit - "Reprinted from The Lancet, Vol. number, Author(s), Title of article, Pages No., Copyright (Year), with permission from Elsevier."

4. Reproduction of this material is confined to the purpose and/or media for which permission is hereby given.

5. Altering/Modifying Material: Not Permitted. However figures and illustrations may be altered/adapted minimally to serve your work. Any other abbreviations, additions, deletions and/or any other alterations shall be made only with prior written authorization of Elsevier Ltd. (Please contact Elsevier at permissions@elsevier.com)

6. If the permission fee for the requested use of our material is waived in this instance, please be advised that your future requests for Elsevier materials may attract a fee.

7. Reservation of Rights: Publisher reserves all rights not specifically granted in the combination of (i) the license details provided by you and accepted in the course of this

https://s100.copyright.com/AppDispatchServlet 
licensing transaction, (ii) these terms and conditions and (iii) CCC's Billing and Payment terms and conditions.

8. License Contingent Upon Payment: While you may exercise the rights licensed immediately upon issuance of the license at the end of the licensing process for the transaction, provided that you have disclosed complete and accurate details of your proposed use, no license is finally effective unless and until full payment is received from you (either by publisher or by $\mathrm{CCC}$ ) as provided in $\mathrm{CCC}^{\prime}$ s Billing and Payment terms and conditions. If full payment is not received on a timely basis, then any license preliminarily granted shall be deemed automatically revoked and shall be void as if never granted. Further, in the event that you breach any of these terms and conditions or any of CCC's Billing and Payment terms and conditions, the license is automatically revoked and shall be void as if never granted. Use of materials as described in a revoked license, as well as any use of the materials beyond the scope of an unrevoked license, may constitute copyright infringement and publisher reserves the right to take any and all action to protect its copyright in the materials.

9. Warranties: Publisher makes no representations or warranties with respect to the licensed material.

10. Indemnity: You hereby indemnify and agree to hold harmless publisher and CCC, and their respective officers, directors, employees and agents, from and against any and all claims arising out of your use of the licensed material other than as specifically authorized pursuant to this license.

11. No Transfer of License: This license is personal to you and may not be sublicensed, assigned, or transferred by you to any other person without publisher's written permission.

12. No Amendment Except in Writing: This license may not be amended except in a writing signed by both parties (or, in the case of publisher, by CCC on publisher's behalf).

13. Objection to Contrary Terms: Publisher hereby objects to any terms contained in any purchase order, acknowledgment, check endorsement or other writing prepared by you, which terms are inconsistent with these terms and conditions or CCC's Billing and Payment terms and conditions. These terms and conditions, together with CCC's Billing and Payment terms and conditions (which are incorporated herein), comprise the entire agreement between you and publisher (and CCC) concerning this licensing transaction. In the event of any conflict between your obligations established by these terms and conditions and those established by CCC's Billing and Payment terms and conditions, these terms and conditions shall control.

14. Revocation: Elsevier or Copyright Clearance Center may deny the permissions described in this License at their sole discretion, for any reason or no reason, with a full refund payable to you. Notice of such denial will be made using the contact information provided by you. Failure to receive such notice will not alter or invalidate the denial. In no event will Elsevier or Copyright Clearance Center be responsible or liable for any costs, expenses or damage incurred by you as a result of a denial of your permission request, other than a refund of the amount(s) paid by you to Elsevier and/or Copyright Clearance Center for denied permissions.

\section{LIMITED LICENSE}

The following terms and conditions apply only to specific license types:

15. Translation: This permission is granted for non-exclusive world English rights only https://s100.copyright.com/AppDispatchServlet 
unless your license was granted for translation rights. If you licensed translation rights you may only translate this content into the languages you requested. A professional translator must perform all translations and reproduce the content word for word preserving the integrity of the article. If this license is to re-use 1 or 2 figures then permission is granted for nonexclusive world rights in all languages.

16. Website: The following terms and conditions apply to electronic reserve and author websites:

Electronic reserve: If licensed material is to be posted to website, the web site is to be password-protected and made available only to bona fide students registered on a relevant course if:

This license was made in connection with a course,

This permission is granted for 1 year only. You may obtain a license for future website posting,

All content posted to the web site must maintain the copyright information line on the bottom of each image,

A hyper-text must be included to the Homepage of the journal from which you are licensing at http://www.sciencedirect.com/science/journal/xxxxx or the Elsevier homepage for books at http://www .elsevier.com, and

Central Storage: This license does not include permission for a scanned version of the material to be stored in a central repository such as that provided by Heron/XanEdu.

17. Author website for journals with the following additional clauses:

All content posted to the web site must maintain the copyright information line on the bottom of each image, and

he permission granted is limited to the personal version of your paper. You are not allowed to download and post the published electronic version of your article (whether PDF or HTML, proof or final version), nor may you scan the printed edition to create an electronic version,

A hyper-text must be included to the Homepage of the journal from which you are licensing at http://www.sciencedirect.com/science/journal/xxxxx , As part of our normal production process, you will receive an e-mail notice when your article appears on Elsevier's online service ScienceDirect (www.sciencedirect.com). That e-mail will include the article's Digital Object Identifier (DOI). This number provides the electronic link to the published article and should be included in the posting of your personal version. We ask that you wait until you receive this e-mail and have the DOI to do any posting.

Central Storage: This license does not include permission for a scanned version of the material to be stored in a central repository such as that provided by Heron/XanEdu.

18. Author website for books with the following additional clauses:

Authors are permitted to place a brief summary of their work online only.

A hyper-text must be included to the Elsevier homepage at http://www.elsevier.com

All content posted to the web site must maintain the copyright information line on the bottom of each image

You are not allowed to download and post the published electronic version of your chapter, nor may you scan the printed edition to create an electronic version.

Central Storage: This license does not include permission for a scanned version of the material to be stored in a central repository such as that provided by Heron/XanEdu. 
19. Website (regular and for author): A hyper-text must be included to the Homepage of the journal from which you are licensing at http://www.sciencedirect.com/science/journal/xxxxx. or for books to the Elsevier homepage at http://www.elsevier.com

20. Thesis/Dissertation: If your license is for use in a thesis/dissertation your thesis may be submitted to your institution in either print or electronic form. Should your thesis be published commercially, please reapply for permission. These requirements include permission for the Library and Archives of Canada to supply single copies, on demand, of the complete thesis and include permission for UMI to supply single copies, on demand, of the complete thesis. Should your thesis be published commercially, please reapply for permission.

\section{Other Conditions}

v1.6

If you would like to pay for this license now, please remit this license along with your payment made payable to "COPYRIGHT CLEARANCE CENTER" otherwise you will be

invoiced within 48 hours of the license date. Payment should be in the form of a check or money order referencing your account number and this invoice number

RLNK500760423.

Once you receive your invoice for this order, you may pay your invoice by credit card. Please follow instructions provided at that time.

Make Payment To:

Copyright Clearance Center

Dept 001

P.0. Box 843006

Boston, MA 02284-3006

For suggestions or comments regarding this order, contact RightsLink Customer Support: customercare@copyright.com or +1-877-622-5543 (toll free in the US) or +1978-646-2777.

Gratis licenses (referencing $\$ 0$ in the Total field) are free. Please retain this printable license for your reference. No payment is required. 
Figure 1.5 and 1.6 Reprint Permissions

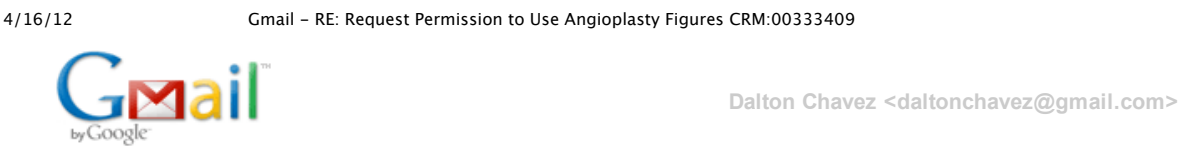

RE: Request Permission to Use Angioplasty Figures CRM:00333409

NHLBI Health Information Center <nhlbiinfo@nhlbi.nih.gov>

Mon, Apr 16, 2012 at 8:45 AM

To: "Chavez, Robert" <rchavez@calpoly.edu>

Dear Mr. Chavez:

Thank you for your inquiry to the National Heart, Lung, and Blood Institute (NHLBI) Health Information Center regarding the NHLBI copyright permission policy.

The text of and information contained in materials published by the National Heart, Lung, and Blood Institute (NHLBI) are in the public domain. No further permission is required to reproduce or reprint the text in whole or in part. This applies to print publications, graphics, and animations in the NHLBl's Health Topics index as well as documents and content from the NHLBI Web site. Organizations may add their own logo or name. The NHLBI asks only that no changes be made to the content of the materials, and that the material as well any NHLBI Internet links not be used in any direct or indirect product endorsement or advertising. Requested sourcing language: Source: National Heart, Lung, and Blood Institute; National Institutes of Health; U.S. Department of Health and Human Services.

We Can!®, The Heart Truth®, COPD: Learn More Breathe Better ${ }^{\circledR}$, and Keep the Beat ${ }^{\mathrm{TM}}$ are trademarks of the U.S. Department of Health and Human Services (HHS). We encourage you to use these brands, logos, and word marks in banners, publications, posters, and promotional materials that promote health programming in your community. However, to maintain the integrity of the messages, tone, and meaning of the logos and word marks we require that they only be used with programs, events, and information whose goals are consistent with the objectives of the NHLBI programs. In addition, we ask that you help us by properly using and crediting HHS trademarks in accordance with the terms of use for We Can! $₫$, The Heart Truth $₫$, COPD: Learn More Breathe Better $®$, and Keep the Beat ${ }^{\mathrm{TM}}$. For approval for use of these HHS program marks, contact the NHLBI at: nhlbiinfo@nhlbi.nih.gov.

Your assistance in making our research and health-related information available to the largest number of people possible is greatly appreciated.

Sincerely,

NHLBI Health Information Center

PO Box 30105

Bethesda, MD 20824

Phone: 301-592-8573

Fax: 301-592-8563

E-mail: nhlbiinfo@nhlbi.nih.gov

Web site: http://www.nhlbi.nih.gov 
April is National Minority Health Month! The NHLBI has a variety of multicultural resources available online and in print. Visit the NHLBl's Health Disparities Web page to view, download, or order these materials.

From: Chavez, Robert

Received: 4/14/2012 8:40 AM

To: Admin User; Information Center, NHLBI; NHLBI Health Information Center

Subject: Request Permission to Use Angioplasty Figures

To Whom It May Concern:

I am a graduate student at California Polytechnic State University, San Luis Obispo (Cal Poly). I am completing my thesis on coronary devices and would like to use two images from the NHLBI website in the introduction of my thesis. I am requesting permission to use the images titled "Coronary Balloon Angioplasty" and "Angioplasty With Stent Placement", which are located at the following URL:

http://www.nhlbi.nih.gov/health/health-topics/topics/angioplasty/howdone.html

An electronic copy of my thesis will be uploaded to my university's online archive of theses and will then be freely available to the public. May I use these two images in my thesis, which will be freely available online?

Thank you for your time.

Sincerely,

Robert Chavez

Biomedical Engineering

Cal Poly, San Luis Obispo

rchavez@calpoly.edu 
Figure 1.7 A and B Reprint Permissions

$4 / 17 / 12$

Gmail - Request Permission to Use Images of IDTE2000

GMail

Dalton Chavez <daltonchavez@gmail.com>

\section{Request Permission to Use Images of IDTE2000}

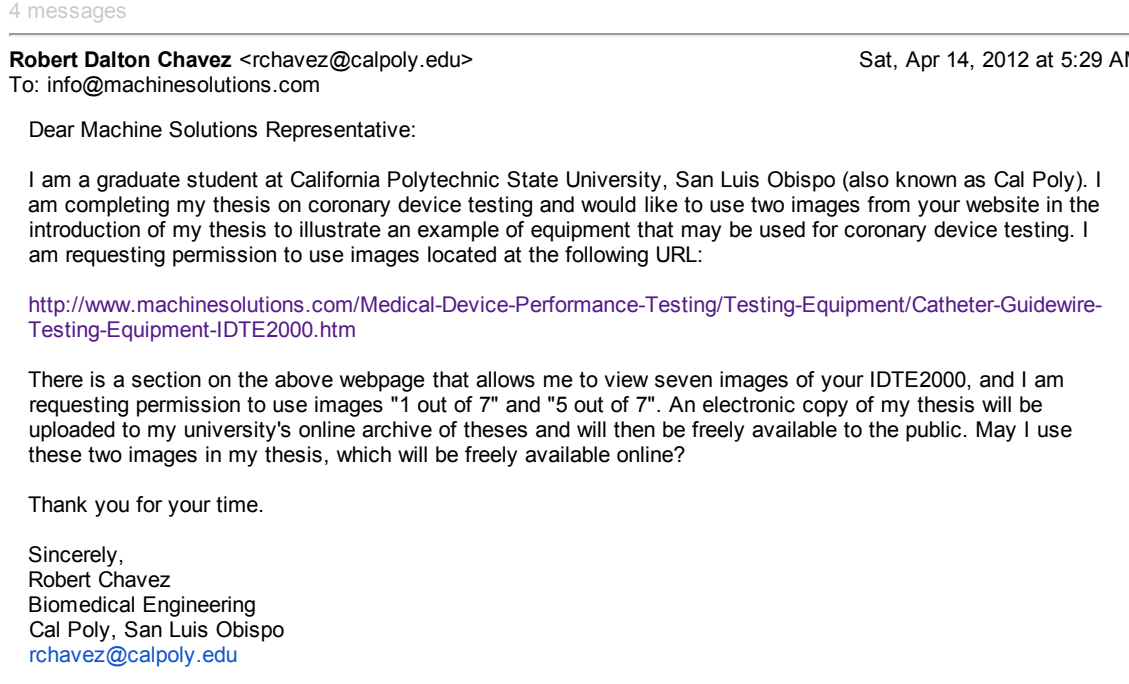

Jason Cronwall <jcronwall@machinesolutions.com>

Sat, Apr 14, 2012 at 9:26 AM

To: Robert Dalton Chavez <rchavez@calpoly.edu>

Robert,

Yes, we approve of that use. If you need anything further, please don't hesitate to contact me.

Best regards,

Jason Cronwall

Director of Global Marketing

Machine Solutions Inc.

+19285563109 Phone

+19285563084 Fax

+1928 2666385 Mobile

icronwall@machinesolutions.com

CONFIDENTIAL: This email (and any attachment) is confidential and may be protected by legal privilege, confidentiality agreements, 
and common or statutory law. If you are not the intended recipient, be aware that any disclosure, copying, distribution or use of this email or any attachment is prohibited. If you have received this email in error, please notify us at once by returning it to the sender and then deleting this copy from your system. Thank you.

From: Robert Dalton Chavez <rchavez@calpoly.edu>

Date: Sat, 14 Apr 2012 05:29:06 -0700

To: <info@machinesolutions.com>

Subject: Request Permission to Use Images of IDTE2000

[Quoted text hidden]

CONFIDENTIAL: This email (and any attachment) is confidential and may be protected by legal privilege, confidentiality agreements, and common or statutory law. If you are not the intended recipient, be aware that any disclosure, copying, distribution or use of this email or any attachment is prohibited. If you have received this email in error, please notify us at once by returning it to the sender and then deleting this copy from your system. Thank you.

Robert Dalton Chavez <rchavez@calpoly.edu $>$

Sun, Apr 15, 2012 at 5:37 AM

To: Jason Cronwall <jcronwall@machinesolutions.com>

Hi Jason,

Thank you very much for your approval! I would like to include your email in an appendix of my thesis as evidence of your approval. May I include your approval email in my thesis? (Your email has a confidentiality statement at the bottom, so I wanted to get your permission to include the email in my thesis.)

Robert

[Quoted text hidden]

Jason Cronwall <jcronwall@machinesolutions.com>

To: Robert Dalton Chavez<rchavez@calpoly.edu>

Tue, Apr 17, 2012 at 3:02 PM

\section{Robert,}

Yes, that's fine.

Best regards,

Jason Cronwall

Director of Global Marketing

Machine Solutions Inc.

+19285563109 Phone

+1928556 3084 Fax

+1928 2666385 Mobile

jcronwall@machinesolutions.com

https: $/ /$ mail.google.com $/ \mathrm{mail} / 2 \mathrm{ui}=2 \& \mathrm{ik}=87 \mathrm{abb} 76 \mathrm{~d} 4 \mathrm{e} \& \mathrm{view}=$ pt $\&$ search $=$ inbox $\&$ th $=136 \mathrm{~b} 0 \mathrm{~d} 402 \mathrm{~d} 82 \mathrm{ff} 89$ 
CONFIDENTIAL: This email (and any attachment) is confidential and may be protected by legal privilege, confidentiality agreements, and common or statutory law. If you are not the intended recipient, be aware that any disclosure, copying, distribution or use of this email or any attachment is prohibited. If you have received this email in error, please notify us at once by returning it to the sender and then deleting this copy from your system. Thank you.

From: Robert Dalton Chavez <rchavez@calpoly.edu>

Date: Sun, 15 Apr 2012 05:37:03-0700

To: Jason Cronwall <jcronwall@machinesolutions.com>

Subject: Re: Request Permission to Use Images of IDTE2000

[Quoted text hidden]

[Quoted text hidden] 


\section{Figure 1.8 Reprint Permissions}

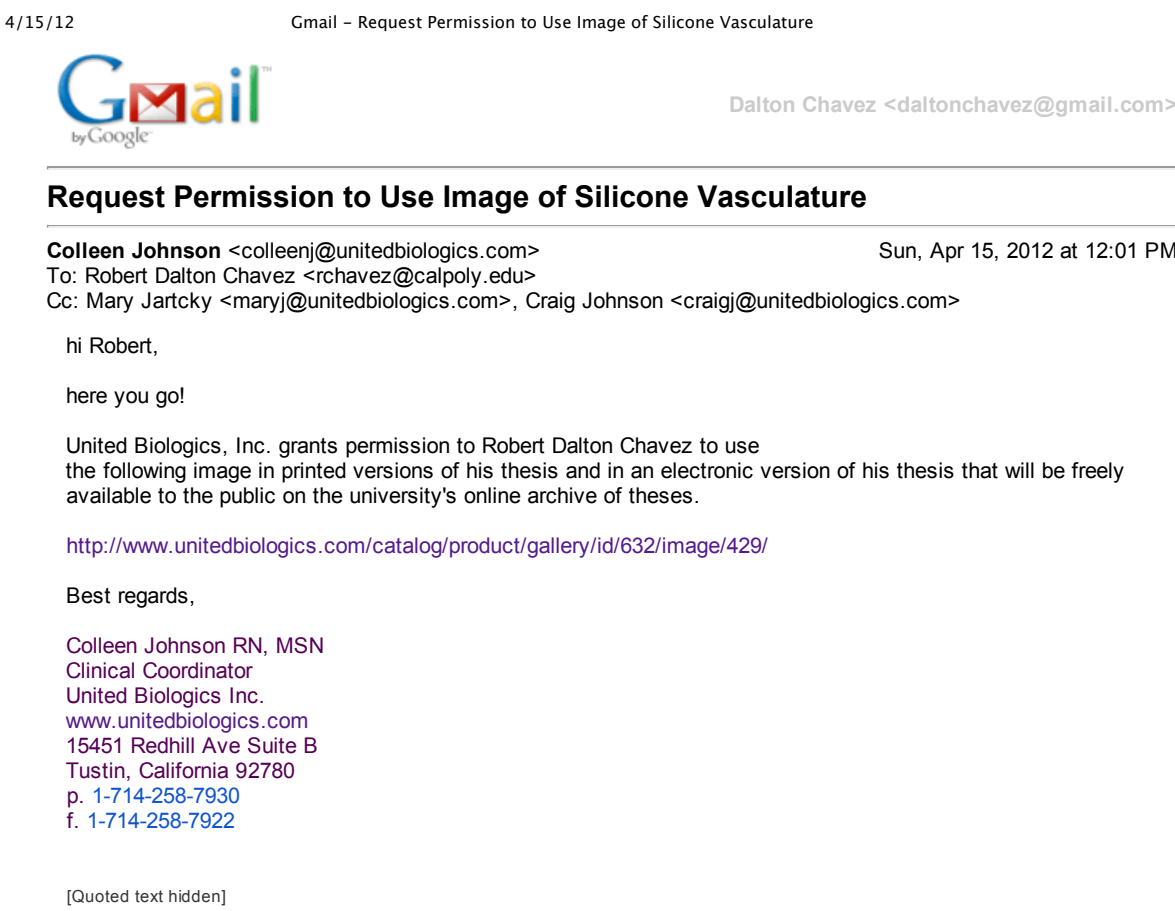

[Quoted text hidden] 
Figure 1.9 A and C Reprint Permissions

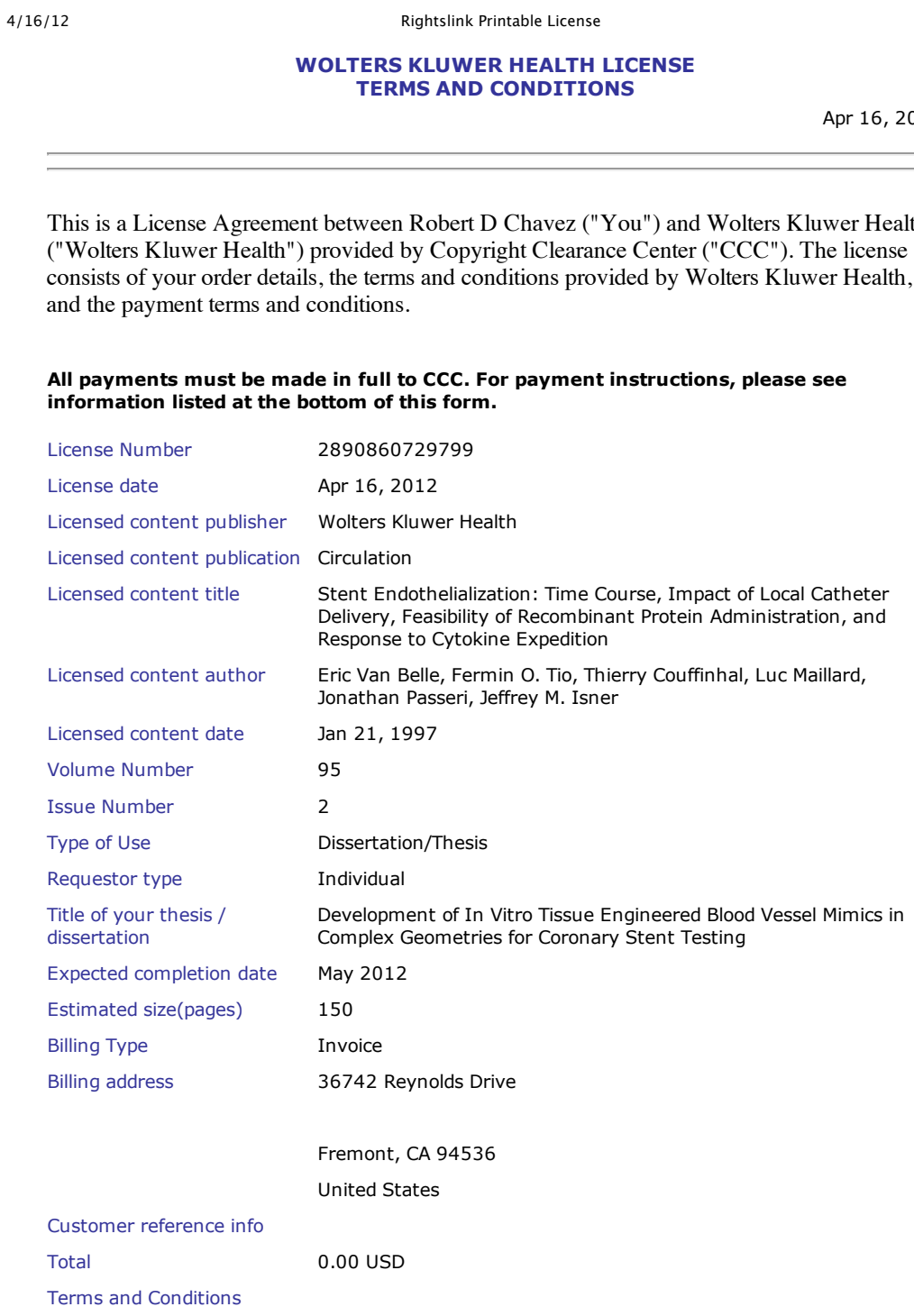

Terms and Conditions 
1. A credit line will be prominently placed and include: for books - the author(s), title of book, editor, copyright holder, year of publication; For journals - the author(s), title of article, title of journal, volume number, issue number and inclusive pages.

2. The requestor warrants that the material shall not be used in any manner which may be considered derogatory to the title, content, or authors of the material, or to Wolters Kluwer.

3. Permission is granted for one time use only as specified in your correspondence. Rights herein do not apply to future reproductions, editions, revisions, or other derivative works. Once term has expired, permission to renew must be made in writing.

4. Permission granted is non-exclusive, and is valid throughout the world in the English language and the languages specified in your original request.

5. Wolters Kluwer cannot supply the requestor with the original artwork or a "clean copy."

6 . The requestor agrees to secure written permission from the author (for book material only).

7. Permission is valid if the borrowed material is original to a Wolters Kluwer imprint (Adis, Lippincott-Raven Publishers, Williams \& Wilkins, Lea \& Febiger, Harwal, Igaku-Shoin, Rapid Science, Little Brown \& Company, Harper \& Row Medical, American Journal of Nursing Co, and Urban \& Schwarzenberg - English Language).

8. If you opt not to use the material requested above, please notify Rightslink within 90 days of the original invoice date.

9. Please note that articles in the ahead-of-print stage of publication can be cited and the content may be re-used by including the date of access and the unique DOI number. Any final changes in manuscripts will be made at the time of print publication and will be reflected in the final electronic version of the issue.

Disclaimer: Articles appearing in the Published Ahead-of-Print section have been peerreviewed and accepted for publication in the relevant journal and posted online before print publication. Articles appearing as publish ahead-of-print may contain statements, opinions, and information that have errors in facts, figures, or interpretation.

Accordingly, Lippincott Williams \& Wilkins, the editors and authors and their respective employees are not responsible or liable for the use of any such inaccurate or misleading data, opinion or information contained in the articles in this section.

10. Other Terms and Conditions:

v1.1

If you would like to pay for this license now, please remit this license along with you payment made payable to "COPYRIGHT CLEARANCE CENTER" otherwise you will be invoiced within 48 hours of the license date. Payment should be in the form of a check or money order referencing your account number and this invoice number RLNK500761317.

Once you receive your invoice for this order, you may pay your invoice by credit card. Please follow instructions provided at that time.

Make Payment To:

Copyright Clearance Center

Dept 001

P.O. Box 843006

Boston, MA 02284-3006

For suggestions or comments regarding this order, contact RightsLink Customer Support: customercare@copyright.com or +1-877-622-5543 (toll free in the US) or +1978-646-2777. 
$4 / 16 / 12$

Rightslink Printable License

Gratis licenses (referencing $\$ 0$ in the Total field) are free. Please retain this printable license for your reference. No payment is required. 
Figure 1.10 Reprint Permissions

$4 / 18 / 12$

Rightslink Printable License

JOHN WILEY AND SONS LICENSE

TERMS AND CONDITIONS

Apr 18, 2012

This is a License Agreement between Robert D Chavez ("You") and John Wiley and Sons ("John Wiley and Sons") provided by Copyright Clearance Center ("CCC"). The license consists of your order details, the terms and conditions provided by John Wiley and Sons, and the payment terms and conditions.

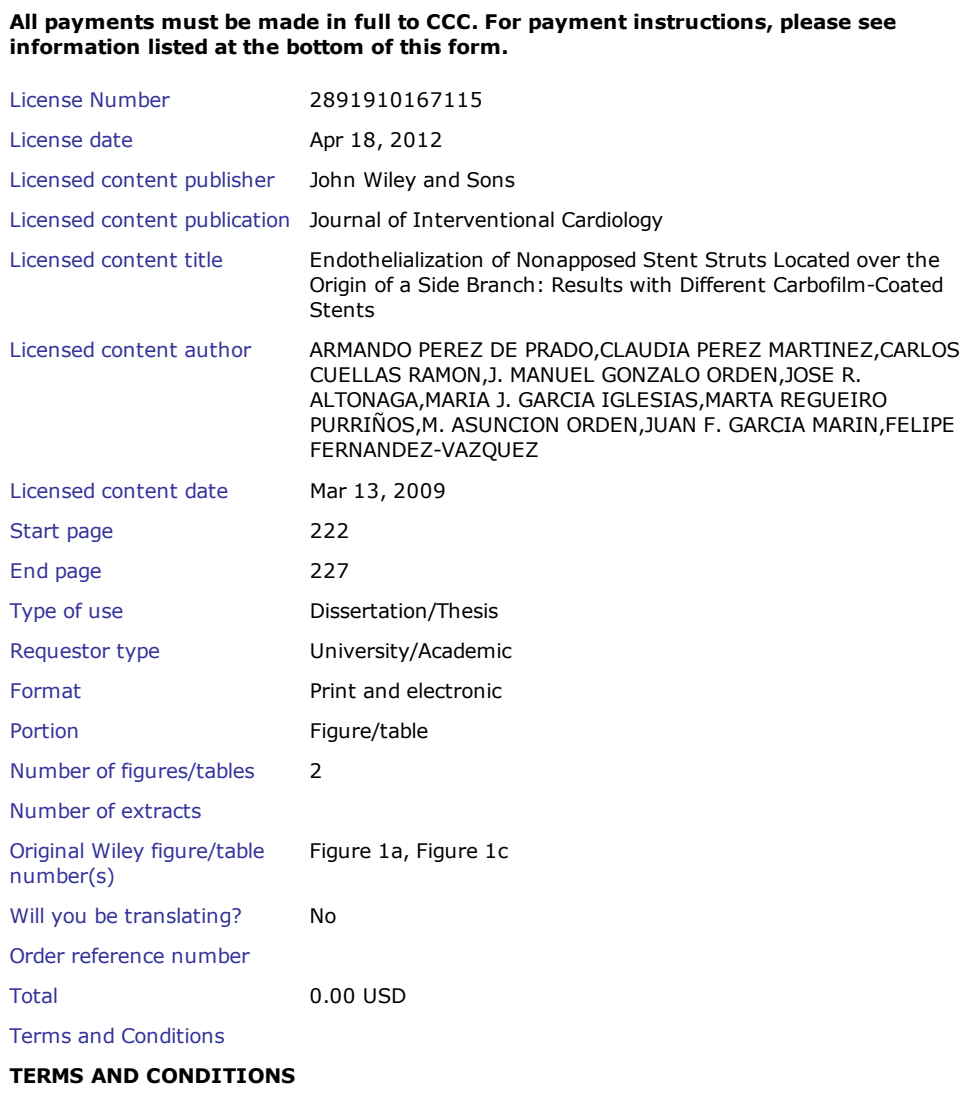

This copyrighted material is owned by or exclusively licensed to John Wiley \& Sons, Inc. or one of its group companies (each a "Wiley Company") or a society for whom a Wiley Company has exclusive 
publishing rights in relation to a particular journal (collectively WILEY"). By clicking "accept" in connection with completing this licensing transaction, you agree that the following terms and conditions apply to this transaction (along with the billing and payment terms and conditions established by the Copyright Clearance Center Inc., ("CCC's Billing and Payment terms and conditions"), at the time that you opened your Rightslink account (these are available at any time at http://myaccount.copyright.com)

Terms and Conditions

1. The materials you have requested permission to reproduce (the "Materials") are protected by copyright.

2. You are hereby granted a personal, non-exclusive, non-sublicensable, non-transferable worldwide, limited license to reproduce the Materials for the purpose specified in the licensing process. This license is for a one-time use only with a maximum distribution equal to the number that you identified in the licensing process. Any form of republication granted by this licence must be completed within two years of the date of the grant of this licence (although copies prepared before may be distributed thereafter). The Materials shall not be used in any other manner or for any other purpose. Permission is granted subject to an appropriate acknowledgement given to the any other purpose. Permission is granted subject to an appropriate acknowledgement given to the author, title of the material/book/journal and the publisher. You shall also duplicate the copyright
notice that appears in the Wiley publication in your use of the Material. Permission is also granted on the understanding that nowhere in the text is a previously published source acknowledged for al or part of this Material. Any third party material is expressly excluded from this permission.

3. With respect to the Materials, all rights are reserved. Except as expressly granted by the terms of the license, no part of the Materials may be copied, modified, adapted (except for minor

reformatting required by the new Publication), translated, reproduced, transferred or distributed, in any form or by any means, and no derivative works may be made based on the Materials without the prior permission of the respective copyright owner. You may not alter, remove or suppress in any manner any copyright, trademark or other notices displayed by the Materials. You may not license, rent, sell, loan, lease, pledge, offer as security, transfer or assign the Materials, or any of the rights granted to you hereunder to any other person.

4. The Materials and all of the intellectual property rights therein shall at all times remain the exclusive property of John Wiley \& Sons Inc or one of its related companies (WILEY) or their respective licensors, and your interest therein is only that of having possession of and the right to reproduce the Materials pursuant to Section 2 herein during the continuance of this Agreement. property rights therein. You shall have no rights hereund above in Section 2. No right, license or interest to any trademark, trade name, service mark or other branding ("Marks") of WILEY or its licensors is granted hereunder, and you agree that you shall not assert any such right, license or interest with respect thereto.

5. NEITHER WILEY NOR ITS LICENSORS MAKES ANY WARRANTY OR REPRESENTATION OF ANY KIND TO YOU OR ANY THIRD PARTY, EXPRESS, IMPLIED OR STATUTORY, WITH RESPECT TO THE MATERIALS OR THE ACCURACY OF ANY INFORMATION CONTAINED IN THE MATERIALS, INCLUDING, WITHOUT LIMITATION, ANY IMPLIED WARRANTY OF MERCHANTABILITY, ACCURACY, SATISFACTORY QUALITY, FITNESS FOR A PARTICULAR PURPOSE, USABILITY, INTEGRATION OR NON-INFRINGEMENT AND ALL SUCH WARRANTIES ARE HEREBY EXCLUDED BY WILEY AND ITS LICENSORS AND WAIVED BY YOU.

6. WILEY shall have the right to terminate this Agreement immediately upon breach of this Agreement by you.

7. You shall indemnify, defend and hold harmless WILEY, its Licensors and their respective directors, officers, agents and employees, from and against any actual or threatened claims, demands, causes of action or proceedings arising from any breach of this Agreement by you.

8. IN NO EVENT SHALL WILEY OR ITS LICENSORS BE LIABLE TO YOU OR ANY OTHER PARTY OR ANY OTHER PERSON OR ENTITY FOR ANY SPECIAL, CONSEQUENTIAL, INCIDENTAL, INDIRECT, EXEMPLARY OR PUNITIVE DAMAGES, HOWEVER CAUSED, ARISING OUT OF OR IN CONNECTION 
WITH THE DOWNLOADING, PROVISIONING, VIEWING OR USE OF THE MATERIALS REGARDLESS OF THE FORM OF ACTION, WHETHER FOR BREACH OF CONTRACT, BREACH OF WARRANTY, TORT, NEGLIGENCE, INFRINGEMENT OR OTHERWISE (INCLUDING, WITHOUT LIMITATION, DAMAGES BASED ON LOSS OF PROFITS, DATA, FILES, USE, BUSINESS OPPORTUNITY OR CLAIMS OF THIRD PARTIES), AND WHETHER OR NOT THE PARTY HAS BEEN ADVISED OF THE POSSIBILITY OF SUCH DAMAGES. THIS LIMITATION SHALL APPLY NOTWITHSTANDING ANY FAILURE OF ESSENTIAL PURPOSE OF ANY LIMITED REMEDY PROVIDED HEREIN.

9. Should any provision of this Agreement be held by a court of competent jurisdiction to be illegal, invalid, or unenforceable, that provision shall be deemed amended to achieve as nearly as possible the same economic effect as the original provision, and the legality, validity and enforceability of the remaining provisions of this Agreement shall not be affected or impaired thereby.

10. The failure of either party to enforce any term or condition of this Agreement shall not constitute a waiver of either party's right to enforce each and every term and condition of this Agreement. No breach under this agreement shall be deemed waived or excused by either party unless such waiver or consent is in writing signed by the party granting such waiver or consent. The waiver by or consent of a party to a breach of any provision of this Agreement shall not operate or be construed as a waiver of or consent to any other or subsequent breach by such other party.

11. This Agreement may not be assigned (including by operation of law or otherwise) by you without WILEY's prior written consent.

12. Any fee required for this permission shall be non-refundable after thirty (30) days from receipt.

13. These terms and conditions together with CCC's Billing and Payment terms and conditions (which are incorporated herein) form the entire agreement between you and WILEY concerning this licensing transaction and (in the absence of fraud) supersedes all prior agreements and representations of the parties, oral or written. This Agreement may not be amended except in writing signed by both parties. This Agreement shall be binding upon and inure to the benefit of the parties' successors, legal representatives, and authorized assigns.

14. In the event of any conflict between your obligations established by these terms and conditions and those established by CCC's Billing and Payment terms and conditions, these terms and conditions shall prevail.

15. WILEY expressly reserves all rights not specifically granted in the combination of (i) the license details provided by you and accepted in the course of this licensing transaction, (ii) these terms and conditions and (iii) CCC's Billing and Payment terms and conditions.

16. This Agreement will be void if the Type of Use, Format, Circulation, or Requestor Type was misrepresented during the licensing process.

17. This Agreement shall be governed by and construed in accordance with the laws of the State of New York, USA, without regards to such state's conflict of law rules. Any legal action, suit or proceeding arising out of or relating to these Terms and Conditions or the breach thereof shall be instituted in a court of competent jurisdiction in New York County in the State of New York in the United States of America and each party hereby consents and submits to the personal jurisdiction of such court, waives any objection to venue in such court and consents to service of process by registered or certified mail, return receipt requested, at the last known address of such party.

\section{Wiley Open Access Terms and Conditions}

All research articles published in Wiley Open Access journals are fully open access: immediately freely available to read, download and share. Articles are published under the terms of the Creative Commons Attribution Non Commercial License. which permits use, distribution and reproduction in any medium, provided the original work is properly cited and is not used for commercial purposes. The license is subject to the Wiley Open Access terms and conditions:

Wiley Open Access articles are protected by copyright and are posted to repositories and websites in accordance with the terms of the Creative Commons Attribution Non Commercial License. At the time of deposit, Wiley Open Access articles include all changes made during peer review, 


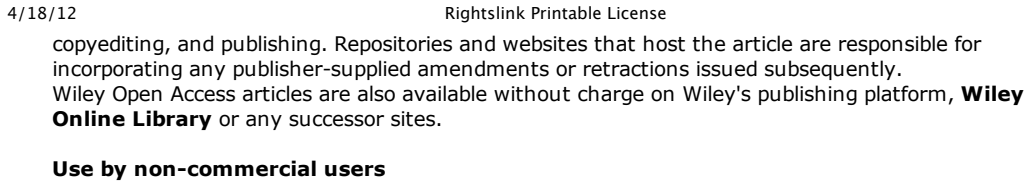

For non-commercial and non-promotional purposes individual users may access, download, copy display and redistribute to colleagues Wiley Open Access articles, as well as adapt, translate, textand data-mine the content subject to the following conditions:

- The authors' moral rights are not compromised. These rights include the right of "paternity" (also known as "attribution" - the right for the author to be identified as such) and "integrity" (the right for the author not to have the work altered in such a way that the author's reputation or integrity may be impugned).

- Where content in the article is identified as belonging to a third party, it is the obligation of the user to ensure that any reuse complies with the copyright policies of the owner of that content.

- If article content is copied, downloaded or otherwise reused for non-commercial research and education purposes, a link to the appropriate bibliographic citation (authors, journal, article title, volume, issue, page numbers, DOI and the link to the definitive published version on Wiley Online Library) should be maintained. Copyright notices and disclaimers must not be deleted.

- Any translations, for which a prior translation agreement with Wiley has not been agreed, must prominently display the statement: "This is an unofficial translation of an article that appeared in a Wiley publication. The publisher has not endorsed this translation."

\section{Use by commercial "for-profit" organisations}

Use of Wiley Open Access articles for commercial, promotional, or marketing purposes requires further explicit permission from Wiley and will be subject to a fee. Commercial purposes include:

- Copying or downloading of articles, or linking to such articles for further redistribution, sale or licensing;

- Copying, downloading or posting by a site or service that incorporates advertising with such content;

- The inclusion or incorporation of article content in other works or services (other than

normal quotations with an appropriate citation) that is then available for sale or licensing, for a fee (for example, a compilation produced for marketing purposes, inclusion in a sales pack)

- Use of article content (other than normal quotations with appropriate citation) by for-profit organisations for promotional purposes

- Linking to article content in e-mails redistributed for promotional, marketing or educational purposes;

- Use for the purposes of monetary reward by means of sale, resale, licence, loan, transfer or other form of commercial exploitation such as marketing products

- Print reprints of Wiley Open Access articles can be purchased from: corporatesales@wiley.com

Other Terms and Conditions: 
4/18/12 Rightslink Printable License

BY CLICKING ON THE "I AGREE..." BOX, YOU ACKNOWLEDGE THAT YOU HAVE READ AND FULLY UNDERSTAND EACH OF THE SECTIONS OF AND PROVISIONS SET FORTH IN THIS AGREEMENT AND THAT YOU ARE IN AGREEMENT WITH AND ARE WILLING TO ACCEPT ALL OF YOUR OBLIGATIONS AS SET FORTH IN THIS AGREEMENT.

v1.7

If you would like to pay for this license now, please remit this license along with your payment made payable to "COPYRIGHT CLEARANCE CENTER" otherwise you will be invoiced with in $\mathbf{4 8}$ hours of the license date. Payment should be in the form of a check or money order referencing your account number and this invoice number RLNK500762984.

Once you receive your invoice for this order, you may pay your invoice by credit card. Please follow instructions provided at that time.

Make Payment To:

Copyright Clearance Center

Dept 001

P.O. Box 843006

Boston, MA 02284-3006

For suggestions or comments regarding this order, contact RightsLink Custome Support: customercare@copyright.com or +1-877-622-5543 (toll free in the US) or +1978-646-2777

Gratis licenses (referencing $\$ 0$ in the Total field) are free. Please retain this printable license for your reference. No payment is required. 